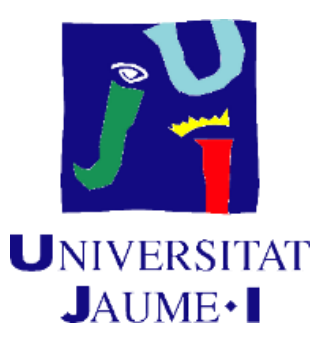

\title{
NEUROINFLAMMATION AND INSULIN RESISTANCE UNDERLY COGNITIVE IMPAIRMENT SEX DIFFERENCES AND POTENTIAL TREATMENTS
}

Sandra Sánchez Sarasúa de la Bárcena

Directora de la tesis:

Dra. Ana María Sánchez Pérez

Junio 2021 
Programa de Doctorado en Ciencias Biomédicas y Salud

Escuela de Doctorado de la Universitat Jaume I

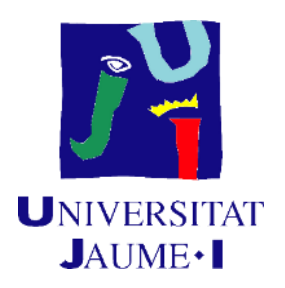

Título de la tesis

\section{Neuroinflammation and insulin resistance underly cognitive impairment. Sex differences and potential treatments}
Memoria presentada por Sandra Sánchez Sarasúa de la Bárcena para optar al grado de doctor/a por la Universitat Jaume I

Nombre y apellidos de la doctoranda:

Sandra Sánchez Sarasúa de la Bárcena

FIRMA original

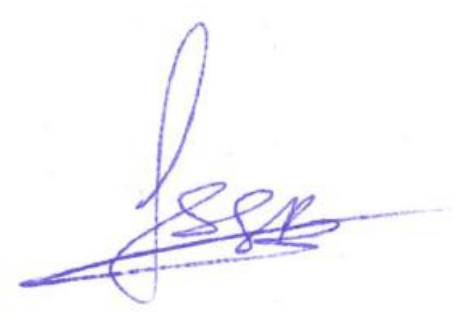

Nombre y apellidos de la directora de la tesis:

Ana María Sánchez Pérez

FIRMA original

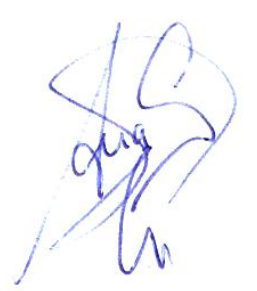

Castelló de la Plana, Junio 2021 


\section{Financiación recibida}

\section{Agencias financiadoras del doctorando/a}

- Contratos predoctorales:

- Beca Programa VALi+d 2016 (ACIF/2016/250) para la contratación de personal investigador en formación de carácter predoctoral (Conselleria d'Educació, Cultura i Esport)

- Fundaciones o agencias que hayan contribuido a financiar gastos formativos o estancias de investigación:

- Beca "EMBO Short-Term Fellowship programme" 2020 para estancias de investigación predoctorales (European Molecular Biology Organization EMBO- Fellowships)

Agencias financiadoras del proyecto de investigación o de los recursos materiales específicos del grupo de investigación

- Plan de promoción de la investigación de la Universitat Jaume I (2014-2017) P1.12014-06

- Plan de promoción de la investigación de la Universitat Jaume I (UJI-B2018-01)

- Ayudas para grupos de investigación consolidables 2015. Conselleria de Educación, Investigación, Cultura y Deporte (GVA AICO/2015/042)

- Crowdfunding Precipita (FECYT) and the Association of Alzheimer Families, AFA

\section{Licencia creative commons (cc)}

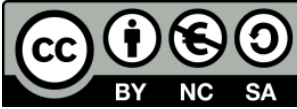


A las Cármenes de mi vida 


\section{ACKNOWLEDGEMENTS}

La familia es lo primero y con ella quiero empezar. Yayo, sé que esto no lo puedes leer pero estoy segura que me acompañas desde allí arriba con una sonrisa, como seguías los partidos de tu equipo, el Castellón CF. Gracias a mis padres y a mi hermano por apoyarme y andar este camino conmigo. Sobre todo a mi madre y a mi abuela, los pilares fundamentales que me han enseñado a ser quien soy hoy, a no rendirme y el sentido del sacrificio, que me han acompañado sin dudar ni cuestionar nada. Gracias por toda esa calma en mitad de tanto ruido.

Gracias Ana por creer en mí como científica, por darme la oportunidad de crecer profesional y personalmente, y por darme las herramientas tanto físicas como mentales. Gracias por la confianza y por sacarme de la zona de confort, he aprendido a luchar contra mis propios fantasmas.

Gracias al profesor Marc Landry por confiar en mí sin apenas conocerme y dejarme formar parte de su equipo durante 3 meses. Ha sido una gran experiencia científica y personal. Merci beaucoup, à bientôt.

Gracias Paco por toda tu ayuda y experiencia. Un placer haber podido aprender de ti.

El camino del doctorado es una carrera de fondo muy dura (ya sé que no digo nada nuevo) pero afortunadamente no se recorre en solitario. He tenido la gran suerte de compartir alegrías y penas con gente a la que, a día de hoy, puedo llamar amigos.

La primera parte de esta tesis tiene dos nombres, dos "madres". Salma y Sandra, Sandra y Salma, team gazpacho en plural. Gracias Salma por enseñarme a formar equipo, un buen equipo, qué fácil nos adaptamos a este mundo donde las dos éramos primerizas pero que peleamos con uñas y dientes. I hope that we will meet again someday, remembering the beginning and being grateful for all that we have reached. Thank you compi.

En ese despacho del segundo piso de medicina hubo un tiempo en el que se formó una pequeña gran familia: the originals. Héctor, Nuno, Alberto y yo como si de un piso compartido se tratase. Gracias Nuno por los buenos ratos y enseñarnos ese "portuñol" que tantas risas nos ha dado. Gracias Héctor, "Tori", por la paciencia y la ciencia que tan bien me enseñaste (y la rision), eres una de las mejores personas que he conocido en ese laboratorio y de verdad que los momentos compartidos contigo tienen un valor gigante. Me llevo un gran amigo para toda la vida. Y desde luego no me podía olvidar de mi gemelier, mi compañero de equipo: Alberto (AAVerto como nombre científico). Tu apoyo en fatigas y alegrías ha allanado este trayecto del doctorado de forma brutal, nuestra conexión hasta en los peores momentos ha hecho que relativizar todo fuese increíblemente fácil. Pocas veces he sentido así de recíproco el alegrarse por los logros del otro. Ojalá nuestros caminos científicos se vuelvan a unir en un futuro pero, si eso no ocurre, se que compartiremos vida estemos donde estemos. Gracias.

A veces en el proceso del doctorado te encuentras sorpresas que te marcan para toda la vida. Sinceramente, nunca pensé que tendría el regalo de conocer a Mary. Gracias por dejarme abrir tu coraza, gracias por tu valentía para abrir la mía. Nunca olvidaré todo lo que he aprendido contigo y de ti. Siguiéndote, perseide, he llegado hasta aquí. Gracias por levantarme cuando ni yo sabía cómo, gracias por tu apoyo incondicional, incluso ahora.

Todavía recuerdo cuando mi buen amigo Andrés me dijo que una chica que conocía estaba interesada en hacer el TFM en el laboratorio de Ana. Yo ahí aún no tenía ni idea del buen tándem que formaríamos Cris y yo. Gracias Cris por los grandes momentos juntas, de risas y no tan risas, por alegrarme los días en ese despacho ( $\mathrm{y}$ en ese animalario también, doñita) y saber complementarme tan bien. Eres una persona de esas que todo el mundo debería conocer y tener cerca, aquí tienes una amiga para todo lo que necesites. 
Gracias Ernestina por tu gran corazón, de verdad que tu espíritu de lucha me desborda. Gracias por tu valentía y sacrificio, contigo he aprendido valores y formas de ver la vida que no conocía. Ojalá que tu constancia y esfuerzo te den una vida llena de alegrías, te lo mereces.

Gracias Vero por tu forma de ser, cabezona (con cariño) pero buena persona y buena madre. Ya sabes que tú eres "la jefa" pero si me necesitas ahí estaré. Gracias también a los recién llegados, Iván y María, que me han recordado esa ilusión que se tiene al empezar en esto de la investigación, sé que os irá muy bien.

Gracias Aroa por tu compañerismo y por estar siempre con una sonrisa, incluso cuando trabajas tan al límite que no tienes tiempo ni de respirar. Solo te diré que el trabajo duro tiene siempre su recompensa, aunque lo veas muy lejano, y en eso no te gana nadie. Este año he tenido la suerte de poder conocerte un poco más y ver lo buena persona y científica que eres. Sé que llegarás lejos.

Gracias Cinta por ser como eres, es decir, como soy yo (ereh tú, fíjate tú). De verdad que pocas personas conozco que compartan mi humor tanto como tú. Nuestro team profesoras pasará a la historia (para bien, espero). Sin moverme de Neurofun, gracias Manoli por toda tu ayuda y consejos, siempre que tenía algún problema sabía que podía contar contigo.

Gracias Bárbara por animarme siempre y estar ahí cuando te necesitaba. Qué bien me sentaban esos ratitos de café o charla en el despacho del primer piso.

No me quiero olvidar de todos los compañeros que han pasado por el laboratorio y que he tenido la suerte de conocer: Mar, Álvaro, Irene, Ana, Hugo. También del departamento de Psicobiología, al que considero mi segunda casa y donde descubrí mi pasión por la neurociencia. Gracias a Laura, Noemí, Isis (ahora dos pisos más arriba), Regu, Andrea y Paula (a ver si pronto un vermut, fuera del animalario mejor). Pero sobre todo tengo que dar las gracias a la única persona que ha estado conmigo en el doctorado de principio a fin. Gracias Carla, doctora Carratalá, porque más que compañera eres amiga y hemos compartido ritmo de laboratorio y ritmo de la noche también. Gracias por estar ahí en ese despacho de la planta baja cuando necesitaba desconectar. Gracias a Paulis y a ti por dejarme ser una "carratonil" más.

Reflexionando, reflexionando...creo que podría asegurar que he pasado la mitad (o más de la mitad) de mi tesis dentro del animalario. Gracias a Alicia, Sandra y Oscar por toda la ayuda y por hacerlo todo tan fácil. Estoy feliz de haberos encontrado en el camino, sois geniales.

Antes he hablado de mi familia de sangre y mi familia científica, pero hay una familia que se elije y que no podía faltar en estos agradecimientos. Aran, mi hermana y mejor amiga, la que cuando me avisa de que viene ese fin de semana ya me cambia el día. Gracias por ser y estar, así de simple. Gracias por ser mi gran apoyo y por compenetrarte conmigo tan bien, siento que estando juntas nada puede salir mal. Cris, no sabes la luz que tienes. Creo que nunca te he dicho la paz que me dan las conversaciones contigo, a veces siento que piensas igual que yo sin haber vivido lo mismo. Gracias por estar ahí siempre, con fe ciega en nuestra amistad. Las dos sois mis compañeras de vida y sé que por muy lejos que estemos siempre os sentiré cerca. Gracias también a Raquel y a mi otra Cris, por crecer juntas en todos los sentidos de la vida, por empujarnos cuando alguna se queda atrás.

Por último, citando una de esas canciones que te ayudan a hacer camino: "brindo, sin más, por no dejarme sola en esto". 


\section{SUMMARY}

Neuroinflammation and type 2 diabetes mellitus (T2DM) are two correlated processes. T2DM patients display insulin resistance: there are normal levels of insulin but the response to it is reduced. Brain insulin resistance and neuroinflammation are important factors contributing to the onset of neurodegenerative diseases, such as Alzheimer's disease (AD). Therefore, molecules that are able to inhibit neuroinflammation could be effective for the prevention and/or treatment of neurological diseases of inflammatory aetiology. Abscisic acid (ABA) is a phytohormone involved in the plant response to hydric stress situations. This compound has also been found in mammals. ABA had been demonstrated to have peripheral anti-inflammatory effect and insulin sensitizing properties. Thus, we hypothesised that ABA chronic administration could have protective effects in a model of neuroinflammation induced by high fat diet (HFD). We confirmed in our model that ABA counteracts peripheral insulin resistance, improving glucose tolerance in HFD-fed animals. Regarding cognitive function, $\mathrm{ABA}$ administration rescued the memory alterations observed in HFD-fed rats. Moreover, we observed that ABA effectively prevented the HFD-induced increase in pro-inflammatory markers (TNF- $\alpha$, APP -Amyloid Precursor Protein- expression and reactive microglia). Furthermore, treatment with ABA counteracted the HFD-induced reduction in BDNF (brain-derived neurotrophic factor) mRNA expression and hippocampal neurogenesis.

Alterations of the insulin receptor (IR) and the insulin substrates (IRS) function is proposed to underlie insulin resistance. In this thesis, we analyzed the effect of HFD and ABA on the expression of substrates 1 and 2 (IRS1, IRS2) in hippocampus. The model of neuroinflammation induced by HFD did not affect the IR, reduced IRS2 and increased IRS1 mRNA levels. This result suggests that IRS1 and IRS2 can be differentially affected by neuroinflammatory processes. Furthermore, ABA restored the HFD-induced changes in IRS1 and IRS2 levels.

In summary, data given in this thesis demonstrate the therapeutic effects of $\mathrm{ABA}$, postulating this phytohormone as a nutraceutical alternative to current anti-inflammatory drugs with adverse effects.

The attention deficit hyperactivity disorder (ADHD) is a neurodevelopmental disorder that affects children although the affection can last through adulthood. Inflammation has been proposed a contributing factor in this disorder, since ADHD patients display high levels of pro-inflammatory cytokines in blood. In an internship at the University of Bordeaux, I studied the anti-inflammatory effects of ABA in an ADHD mouse model produced by 6-OHDA neonatal lesion. In this pilot study, female and male mice seem to display differences in ADHD symptomatology. Furthermore, ABA administration appeared to also affect differentially males and females, almost opposite in certain parameters like pain sensitivity. Although these preliminary data need to be confirmed (by increasing the subjects) this pilot study points to a revision of disease preclinical models and pharmacological treatments in comparative females and males studies.

The specific and distinct role of IRS1 and IRS2 in distinct brain networks, isolated from other comorbidities such as chronic inflammation or AD linked genetic mutations, has not been fully established. To that end, we have used of adeno-associated virus (AAV) expressing short hairpin ribonucleic acid (shRNA) targeting IRS1 (shIRS1) into the dorsal HPC of female and male rats. We found that female rats inoculated with shIRS1 showed spatial and recognition memory deficits but males only showed deficits in the spatial memory task. This result suggests that the female brain may display a higher susceptibility to insulin resistance caused by IRS1 deficits. In post-mortem analysis, we observed that the viral particle had mainly infected somatostatin (SOM)positive and parvalbum (PV)-positive neurons in the hippocampal dentate gyrus. Accordingly, we found AAV fibres in the medial septum (MS) from the long projecting SOM hilar neurons. In the MS, we observed that synaptophysin labelled puncta on shIRS1 AAV fibres were reduced compared to control, thus suggesting an impaired synaptic plasticity due to IRS1 reduction. Hippocampal SOM regulation of septal PV neurons is crucial in the regulation of septo-hipocampal circuitries in memory formation. Our data shows that IRS1 is required for proper neuronal activity, since in the absence of neuroinflammation IRS1 knockdown can cause cognitive deficits. 


\section{TABLE OF CONTENTS}

1.1. INSULIN SIGNALLING IN BRAIN: RECEPTOR AND SUBSTRATES

1.1.1. Insulin transport and receptor functions 1

1.1.2. Insulin receptor substrates 3

1.2. NeUROINFLAMMATION AND INSULIN RESISTANCE 6

1.3. ABSCISIC ACID: INSULIN SENSITIZER AND ANTI-INFLAMMATORY NUTRACEUTICAL 9

1.4. BRAIN NETWORKS: THE MEMORY CIRCUITRY

2. HYPOTHESIS \& OBJECTIVES

3. CHAPTER 1: THE EFFECT OF ABSCISIC ACID CHRONIC TREATMENT ON NEUROINFLAMMATORY MARKERS AND MEMORY IN A RAT MODEL OF HIGH-FAT DIET INDUCED NEUROINFLAMMATION

$\begin{array}{ll}\text { 3.1. INTRODUCTION } & 17\end{array}$

3.2. METHODS 18

3.3. RESULTS

3.3.1. ABA TREATMENT IMPROVES GLUCOSE TOLERANCE OF HFD-FED ANIMALS 21

3.3.2. ABA RESTORES MEMORY IMPAIRMENT INDUCED BY HFD 22

3.3.3. ABA ADMINISTERED IN THE DRINKING WATER CAN CROSS THE BBB 24

3.3.4. HFD INTAKE INCREASES TNFA AND REACTIVE MICROGLIA LEVELS IN HYPOTHALAMUS. ABA CAN EFFECTIVELY COUNTERACT THESE CHANGES

$\begin{array}{ll}\text { 3.4. DISCUSSION } & 27\end{array}$

4. CHAPTER 2: ABSCISIC ACID SUPPLEMENTATION RESCUES HIGH FAT DIET-INDUCED ALTERATIONS IN HIPPOCAMPAL INFLAMMATION AND IRSS EXPRESSION

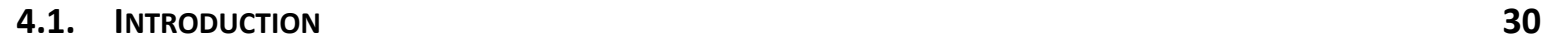

\begin{tabular}{lr} 
4.2. & 31 \\
\hline
\end{tabular}

\begin{tabular}{lr} 
4.3. & RESULTS \\
\hline
\end{tabular}

4.3.1. A HFD ALTERS HIPPOCAMPAL IRS1 AND IRS2 MRNA EXPRESSION. ABA TREATMENT CAN PREVENT THESE $\begin{array}{ll}\text { CHANGES } & 33\end{array}$

4.3.2. ABA CAN EFFECTIVELY RESTORE HFD-INDUCED REDUCTION IN HIPPOCAMPAL IRS2 PROTEIN 33

4.3.3. ABA RESCUES THE HFD-INDUCED REDUCTION IN HIPPOCAMPAL NEUROGENESIS

4.3.4. IRS1 AND IR21 MRNA LEVELS CORRELATE DIFFERENTLY WITH TNFA, BDNF AND APP 35

4.3.5. THE IRS1 EXPRESSION CORRELATES WITH WEIGHT GAIN BUT IRS2 DOES NOT 36

$\begin{array}{lr}\text { 4.4. DISCUSSION } & 37\end{array}$

5. CHAPTER 3: AAV DELIVERY OF SHRNA AGAINST IRS1 IN GABAERGIC NEURONS IN RAT HIPPOCAMPUS IMPAIRS SPATIAL MEMORY IN FEMALES AND MALE RATS 40

5.1. INTRODUCTION 
5.2. Materials AND Methods

5.3. RESULTS 44

5.3.1. SHRNA IRS1 KNOCKS DOWN IRS1 MRNA LEVELS IN HEK293 CELLS

5.3.2. AAV-SHIRS1-EGFP INJECTION IN DORSAL HIPPOCAMPUS ALTERS SPATIAL MEMORY, ESPECIALLY IN FEMALES, BUT NOT SOCIAL MEMORY, NOR ANXIETY OR DEPRESSIVE SYMPTOMS

5.3.3. AAV DJ8 TRACKS DORSAL HIPPOCAMPUS EFFERENT CONNECTIONS 46

5.3.4. AAV-DJ8 LABELS DORSAL SPECIFIC HIPPOCAMPAL NEURONS 46

5.3.5. AAV DJ8 AXONS IN THE MEDIAL SEPTUM REACH PV AND CHAT OCCUPIED AREAS

5.3.6. AAV-SHIRS1 RESULTS IN LOWER EXPRESSION OF IRS1 IN THE DORSAL HIPPOCAMPUS AND CORRELATES WITH BEHAVIOUR $\quad 48$

5.3.7. AAVSHIRS1 INFECTION RESULTS IN LOWER SYNAPTOPHYSIN POSITIVE LABELS COMPARED TO AAV CONTROL $\quad 49$

5.4. DISCUSSION $\quad 51$

6. CHAPTER 4: TARGETING NEUROINFLAMMATION IN A MICE MODEL OF ADHD WITH

ALTERED PAIN SENSITIVITY. A PILOT STUDY $\quad 55$

6.1. INTRODUCTION

6.2. MATERIALS AND METHODS

$\begin{array}{ll}\text { 6.3. RESULTS } & \mathbf{5 8}\end{array}$

6.3.1. 6-OHDA LESION EFFECT ON WEIGHT INCREMENT AND WATER CONSUMPTION. EVALUATION OF ABA TREATMENT

6.3.2. 6-OHDA LESION EFFECT ON IMPULSIVITY

6.3.3. 6-OHDA LESION EFFECT ON THE SPONTANEOUS LOCOMOTOR HYPERACTIVITY. EVALUATION OF ABA $\begin{array}{ll}\text { TREATMENT } & 60\end{array}$

6.3.4 6-OHDA LESION EFFECT ON PAIN HYPERSENSITIVITY. EVALUATION OF ABA TREATMENT 61

$\begin{array}{ll}\text { 6.4. DISCUSSION } & 64\end{array}$

7. GENERAL DISCUSSION AND FUTURE DIRECTIONS $\quad 67$

7.1. THE POTENTIAL therapeutic VAlUe OF ABA, IN MALE RODENTS 67

7.2. INSULIN RESISTANCE IMPAIRS SPATIAL AND RECOGNITION MEMORY IN FEMALES, ONLY SPATIAL MEMORIES IN MALES

$\begin{array}{ll}\text { 7.3. ADHD MODEL: POTENTIAL SEX-DIFFERENCES } & 70\end{array}$

8. CONCLUSIONS

9. BIBLIOGRAPHY $\quad 74$

10. ANNEXES 100

10.1. SUPPLEMENTARY INFORMATION 100

10.1.1. Cell transfection and RT-qPCR 100

$\begin{array}{ll}\text { 10.2. SUPPLEMENTARY FIGURES } & 101\end{array}$

$\begin{array}{lr}\text { 10.3. SUPPLEMENTARY TABLES } & 107\end{array}$

$\begin{array}{lr}\text { 10.4. PUBLICATIONS } & 109\end{array}$ 


\section{FIGURES AND TABLES LIST}

\section{GENERAL INTRODUCTION}

FIG. 1.1. IRS1 AND IRS2 PROTEIN DOMAINS.

FIG. 1.2. INSULIN RECEPTOR SUBSTRATES (IRS) DISTRIBUTION THROUGHOUT THE MOUSE BRAIN

FIG. 1.3. SCHEMATIC REPRESENTATION OF SOME SERINE PHOSPHORYLATION SITES IN IRS1 AND POTENTIAL FUNCTIONS (IN HUMANS).

FIG. 1.4. INSULIN SIGNALLING STIMULATES IRS1/2 TYROSINE (TYR) PHOSPHORYLATION (HEALTHY CONDITION). INSULIN RESISTANCE INDUCED BY IRS1/2 SERINE/THREONINE (SER/THR) PHOSPHORYLATION (INFLAMMATORY CONDITION).

FIG. 1.5. SCHEMATIC HIPPOCAMPAL EFFERENT/AFFERENT CONNECTIONS.

\section{CHAPTER 1: THE EFFECT OF ABSCISIC ACID CHRONIC TREATMENT ON}

NEUROINFLAMMATORY MARKERS AND MEMORY IN A RAT MODEL OF HIGH-FAT DIET INDUCED

NEUROINFLAMMATION

FIG. 3.1. EXPERIMENT DESIGN

TABLE 3.1. COMPOSITION OF HIGH FAT DIET

FIG. 3.2. BODY WEIGHT AND WATER, FOOD AND ABA INTAKE

FIG. 3.3. INTRAPERITONEAL GLUCOSE TOLERANCE TEST

FIG. 3.4. BEHAVIORAL DATA OF NOVEL OBJECT RECOGNITION (NOR) 23

FIG. 3.5. BEHAVIORAL DATA OF T-MAZE

TABLE 3.2. ABA QUANTIFICATION BY HPLC

FIG. 3.6. REACTIVE MICROGLIA LEVELS IN HYPOTHALAMUS

Fig. 3.7. CYTOKINE MRNA LEVELS IN THE HYPOTHALAMUS

4. CHAPTER 2: ABSCISIC ACID SUPPLEMENTATION RESCUES HIGH FAT DIET-INDUCED

ALTERATIONS IN HIPPOCAMPAL INFLAMMATION AND IRSS EXPRESSION

TABLE 4.1. PRIMERS SEQUENCES

TABLE 4.2. HIPPOCAMPAL MRNA EXPRESSION OF NEUROINFLAMMATION MARKERS AND INSULIN/IGF-1 PATHWAY GENES

FIG. 4.1. WESTERN BLOT ANALYSIS EVALUATING THE EXPRESSION OF PROTEINS IN THE HIPPOCAMPUS

FIG. 4.2. ABA RESCUES THE HFD-INDUCED REDUCTION IN THE NUMBER OF DCX-POSITIVE NEURONS IN THE HIPPOCAMPUS

FIG. 4.3. THE CORRELATION OF IRS1 AND IRS2 WITH TNFA, APP, AND BDNF 36

FIG. 4.4. THE CORRELATION OF IRS1 AND IRS2 VERSUS THE PERCENTAGE OF WEIGHT GAIN 36

5. CHAPTER 3: AAV DELIVERY OF SHRNA AGAINST IRS1 IN GABAERGIC NEURONS IN RAT HIPPOCAMPUS IMPAIRS SPATIAL MEMORY IN FEMALES AND MALE RATS

$\begin{array}{lr}\text { FIG. 5.1. EXPERIMENT DESIGN } & 44\end{array}$

FIG. 5.2. AAV SHIRS1 IMPAIRS SPATIAL MEMORY $\quad 45$

FIG. 5.3. AAV DJ8 TRACES DORSAL HIPPOCAMPUS EFFERENTS $\quad 46$

FIG. 5.4. AAV DJ8 LABELS DORSAL SPECIFIC HIPPOCAMPAL NEURONS

FIG. 5.5. AAV-LABELED AXONS IN THE MEDIAL SEPTUM $\quad 48$

FIG. 5.6. SILENCING EFFECT OF SHIRS1 IN THE DORSAL HIPPOCAMPUS $\quad 49$

FIG. 5.7. AAV-CMV-SHIRS1-EGFP INOCULATION IN THE DORSAL HIPPOCAMPUS RESULTS IN LOWER

SYNAPTOPHYSIN POSITIVE LABELS IN FIBERS REACHING MEDIAL SEPTUM

50 
FIG. 6.1. SCHEDULE OF THE EXPERIMENT DESIGN

FIG. 6.2. BODY WEIGHT AND WATER, FOOD AND ABA INTAKE

$\begin{array}{ll}\text { FIG. 6.3. IMPULSIVITY } & 60\end{array}$

FIG. 6.4. SPONTANEOUS LOCOMOTOR HYPERACTIVITY $\quad 61$

Fig. 6.5. THERMAL STIMULATION (PAIN THRESHOLD) $\quad 62$

Fig. 6.6. MECHANICAL STIMULATION (PAIN THRESHOLD) 63

10. ANNEXES

100

FIG. S5.1. SEQUENCES OF THE OLIGONUCLEOTIDES USED FOR THE TWO SHRNA CONSTRUCTS SELECTED AS SHIRS1 CANDIDATES

101

FIG. S5.2. SILENCING EFFECT OF SHIRS1 IN CULTURED CELLS $r$

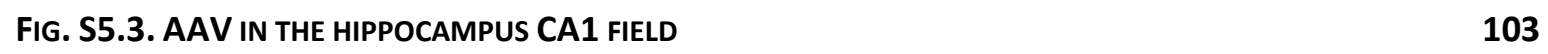

FIG. S5.4. AAV DJ8 DOES NOT COLOCALIZE WITH GFAP-POSITIVE ASTROCYTES $\quad 104$

FIG. S5.5. CORRELATION BETWEEN T-MAZE BEHAVIOUR (DI) AND IRS1 KNOCKDOWN 105

FIG. S5.6. WESTERN BLOT ANALYSIS OF HEK CELLS TRANSFECTED WITH SHIRS1 PLASMID OR CONTROL PLASMID

106

$\begin{array}{ll}\text { TABLE S5.1. WEIGHT BEFORE AND AFTER SHIRS1 INOCULATION } & 107\end{array}$

$\begin{array}{ll}\text { TABLE S5.2. PRIMERS SEQUENCES } & 108\end{array}$ 


\section{ABBREVIATIONS LIST}

5-HT 5-hydroxytryptamine (serotonin)

6-OHDA 6-hydroxydopamine

\section{A}

A $\beta$ Amyloid-beta

AAV Adeno-associated virus

ABA Abscisic acid

Acb Nucleus accumbens

AD Alzheimer's disease

ADHD Attention deficit hyperactivity disorder

AgRP Agouti-related peptide

AMPA $\alpha$-amino-3-hydroxy-5-methyl-4isoxazolepropionic acid

AMPK Adenosine monophosphate-activated protein kinase

ANOVA Analysis of variance

APOE Apolipoprotein E

APP Amyloid-beta precursor protein

B

BAX Bcl-2-associated X

BBB Blood-brain barrier

BDNF Brain-derived neurotrophic factor

C

CA Cornu Ammonis

CA1 Cornu Ammonis 1

CA3 Cornu Ammonis 3

cADPR Cyclic adenosine difosfato-ribose

cAMP Cyclic adenosine monophosphate
CAR Cliff avoidance reaction

CaRT Cocaine- and amphetamine-regulated transcript

Cb Cerebellum

CB Calbindin

ChAT Choline acetyltransferase (Cholinergic)

CNS Central nervous system

CPu Caudate putamen

CR Calretinin

CREB cAMP response element-binding

CRH Corticotrophin-releasing hormone

CSF Cerebrospinal fluid

\section{D}

DA Dopamine

DAB 3,3'-diaminobenzidine

DAMPs Damage-associated molecular patterns

DAT Dopamine transporter

DCX Doublecortin

DG Dentate gyrus

DI Discrimination index

DPX Dibutylphthalate polystyrene xylene

DS Dorsal subiculum

\section{$\mathbf{E}$}

EC Entorhinal cortex

EGFP Enhanced green fluorescent protein

ER Endoplasmic reticulum 
ERK Extracellular signal-regulated kinase

\section{$\mathbf{F}$}

f Fornix

FoxO Forkhead box O

FST Forced swim test

\section{G}

GABA Type A $\gamma$-aminobutyric acid

GFAP Glial fibrillary acidic protein

GL Granular layer

GLP1 Glucagon-like peptide 1

GLUT4 Glucose transporter 4

GLUT8 Glucose transporters 8

GLUTs Glucose transporters

GPCR G-protein coupled receptor

Grb Growth factor receptor-binding protein

GSK3ß Glycogen synthase 3-beta

\section{H}

HDB Horizontal limb diagonal band

HEK Human embryonic kidney cells

HFD High fat diet

HIL Hilar somatostatin interneurons with long axons

HIPP Hilar somatostatin interneurons

HPC Hippocampus

HPLC High-performance liquid chromatography

Hyp Hypothalamus
Iba1 Ionized calcium-binding adaptor molecule 1

icv Intracerebroventricular

IDE Insulin-degrading enzyme

IFN- $\gamma$ Interferon gamma

IGF-1 Insulin-like growth factor-1

IGF-1R Insulin-like growth factor-1 receptor

IKK $\boldsymbol{\beta}$ Inhibitor of nuclear factor kappa-B kinase subunit beta

IL Interleukin

IN Intranasal

IP Intraperitoneal

IR Insulin receptor

IRed Infrared

IRS Insulin receptor substrates

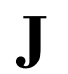

JNK c-Jun N-terminal kinase

\section{$\mathbf{K}$}

KRLB Kinase regulatory-loop binding domain

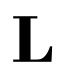

LANC Lanthionine synthetase component C

LANCL2 Lanthionine synthetase component C-like protein 2

LEC Lateral entorhinal cortex

LPS Lipopolysaccharides

LTD Long-term depression

LTP Long-term potentiation 


\section{M}

M1 Pro-inflammatory macrophage phenotype

M2 Anti-inflammatory macrophage phenotype

MCI Mild cognitive impairment

MCP-1 Monocyte chemoattractant protein-1

MDA Malondialdehyde

MDD Major depressive disorder

MEC Medial entorhinal cortex

MEK Mitogen-activated protein kinase

MM Mammillary bodies

mL Mililiter

ML Molecular layer

MS Medial septum

mTOR Mammalian target of rapamycin

mTOR-S6K Mammalian target of rapamycin-ribosomal protein S6 kinase

mTORC1 Mammalian target of rapamycin complex 1

\section{$\mathbf{N}$}

NFк及 Nuclear factor kappa-light-chainenhancer of activated B cells

NGF Nerve growth factor

NLR Nucleotide-binding oligomerization domain-like receptor

NLRP3 Nucleotide-binding oligomerization domain-like receptor family pyrin domain containing 3

NMDA N-methyl-D-aspartate

NO Nitric oxide
NOD Nucleotide-binding oligomerization domain-like receptor

NOR Novel object recognition

NPY Neuropeptide Y

NSAIDS Non-steroidal anti-inflammatory drugs

\section{$\mathbf{P}$}

P85 Regulatory subunit of phosphoinositide 3-kinase

pAKT Phosphorylated AKT

PAMPs Pathogen-associated molecular patterns

PBS Phosphate buffered saline

PC Perirhinal cortex

PD Parkinson's disease

PDK1 3-phosphoinositide-dependent protein kinase 1

pERK Phosphorylated extracellular signalregulated kinase

PFA Paraformaldehyde

PFC Prefrontal cortex

pfp Perforant pathway

PH Pleckstrin homology

PI3K Phosphatidylinositol 3-kinase

pIKK Phosphrorylated inhibitor of nuclear factor kappa-B kinase subunit

PIP2 Phosphatidylinositol 4,5-bisphosphate

PIP3 Phosphatidylinositol $(3,4,5)$-trisphosphate

PKA Protein kinase A

PKC Protein kinase C

PoDG Polymorphic area of the dentate gyrus 
POMC Proopiomelanocortin

PPAR $\gamma$ Peroxisome proliferator-activated receptor gamma

PRRs Pattern recognition receptors

PS1 Presenilin 1

PSD-95 Postsynaptic density protein 95

pTAU Phosphorylated Tau

PTB Phosphotyrosine binding

PV Parvalbumin

\section{$\mathbf{R}$}

RA Retinoic acid

RAR $\boldsymbol{\alpha}$ Retinoic acid receptor alpha

RN Raphe nucleus

RT Room temperature

RXRs Retinoid X receptors

\section{S}

sc Subcutaneous

SD Standard diet

SEM Standard error of mean

Ser Serine

SGZ Subgranular zone

SH Src homology domain

SH2 Src homology 2 domain

She Src homology and collagen

shIRS1 Short hairpin ribonucleic acid targeting insulin receptor substrate 1

shRNA Short hairpin ribonucleic acid

SHP-2 Src homology domain-containing phosphatase-2
SHPTP-2 Src homology 2 domain protein tyrosine phosphatase

Sol Solitary tract nucleus

SOM Somatostatin

SOS Son-of sevenless

SuM Supramammillary nucleus

Synap Synaptophysin

\section{$\mathbf{T}$}

T1DM Type 1 diabetes mellitus

T2DM Type 2 diabetes mellitus

TB Tris buffer

TBS Tris-buffered saline

Thr Threonine

TLRs Toll-like receptors

TNF $\alpha$ Tumor necrosis factor alpha

TNFR Tumor necrosis factor receptor

TrkB Tropomyosin receptor kinase B

Tyr Tyrosine

TZDs Thiazolidinediones

\section{V}

VDB Vertical limb diagonal band

VEH Vehicle

VTA Ventral tegmental area 
1

GENERAL INTRODUCTION 


\subsection{Insulin signalling in brain: receptor and substrates}

\subsubsection{Insulin transport and receptor functions}

Since brain can use glucose independently of insulin, brain was considered for a long time an insulin insensitive tissue. However, the discovery of the insulin receptor (IR) in the 70's ${ }^{[1]}$ changed this view, and nowadays, insulin signalling important role in brain function is widely recognized. Insulin is an endocrine hormone mainly synthesized and secreted by the pancreatic $\beta$-cells ${ }^{[2]}$. The source of brain insulin was debated for a long time, but now accumulated evidence shows that, although pancreatic insulin crosses the blood-brain barrier (BBB) by means of a saturable transporter there is also local insulin synthesis ${ }^{[3]}$. The insulin transporter is localized in the brain capillary endothelial cells, in this way insulin crosses from blood plasma to brain interstitial fluid and then to cerebrospinal fluid (CSF) ${ }^{[4]}$. Insulin transport trough the BBB can be affected by different conditions, as has been reported in rodent models of diabetes ${ }^{[5]}$ and aging ${ }^{[6]}$. Insulin transport rate may also depend on the brain area. Thus, early studies suggested that the olfactory bulb has very high insulin transport and insulin degradation rate ${ }^{[7]}$, also the hypothalamus (Hyp) and cortex ${ }^{[8]}$, whereas no detectable transport reported in thalamus, midbrain or occipital cortex ${ }^{[8]}$.

Peripherally, the principal role of insulin is to induce blood glucose uptake into muscle adipose tissue and liver, after food intake when there is a glucose increase in blood ${ }^{[10]}$. Glucose uptake is mediated by the transmembrane glucose transporters (GLUTs). The main insulinsensitive glucose transporters are GLUT4 and the more recently discovered member, GLUT8. Once insulin stimulates its receptor, insulin-dependent glucose transporters are translocated to the plasma membrane by an Akt2-mediated signalling. The expression of GLUT4 is reduced in the adipose tissue of type 2 diabetes mellitus (T2DM) patients, contributing to reduced glucose uptake by these cells ${ }^{[11]}$.

Centrally, GLUT4 co-expresses with IR in Hyp, cerebellum ${ }^{[12]}$, and hippocampus (HPC), where it has been demonstrated that the mechanism of translocation is similar to peripheral, through Akt cascade ${ }^{[13]}$. GLUT4 seems to be specifically expressed in neuronal soma and dendrites. At least in rat HPC, GLUT4 labelling has only been found in neuronal but not in glial cells ${ }^{[14]}$. Moreover, GLUT4 expression seems specific of some types of neurons. For example, in rat basal forebrain, cholinergic (ChAT) and parvalbumin (PV), but no calbindin (CB) and calretinin (CR) neurons, express GLUT4 ${ }^{[15]}$. On the other hand, GLUT8 is distributed widespread in the rat brain ${ }^{[16]}$. Similarly to GLUT4, GLUT8 was found specifically in the cell body and proximal apical dendrites of hippocampal rat neurons but not in astroglia or microglial cells ${ }^{[16]}$. Unlike GLUT4, GLUT8 is not expressed in the plasma membrane but in endoplasmic reticulum (ER), endosomes and lysosomes membranes. Interestingly, elevated plasma glucose levels increases GLUT8 association with ER, providing glucose availability for protein glycosylation in the ER lumen ${ }^{[17,18]}$.

One of the best well-known insulin effects in brain is the regulation of food intake and energy balance. In the hypothalamus, insulin downregulates orexigenic peptides: neuropeptide $Y$ (NPY) and Agouti-related peptide (AgRP). Simultaneously, insulin upregulates the anorexigenic peptides: proopiomelanocortin (POMC), and the cocaine-amphetamine-regulated transcript $(\mathrm{CaRT})$. Thus, the disruption of IR signalling results in increased appetite and food intake, leading to obesity ${ }^{[19,20]}$.

Most relevant to this thesis, brain insulin resistance and metabolic syndrome leads to accelerated cognitive decline and increases the risk of developing Alzheimer's disease (AD) ${ }^{[21]}$. Neuroimaging studies in T2DM patients displayed hippocampal and amygdala atrophy together with altered functional connectivity with other brain regions ${ }^{[22-24]}$. 
Preclinical studies with diabetic rats show long-term potentiation (LTP) (see note ${ }^{1}$ ) alterations due to diminished expression of the $\alpha$-amino-3-hydroxy-5-methyl-4-isoxazolepropionic acid (AMPA) and N-methyl-D-aspartate (NMDA) receptors at the postsynaptic density ${ }^{[25]}$. Conversely, insulin has been shown to induce LTP in mouse hippocampal slices ${ }^{[26]}$. The upregulation of LTP can be due to insulin action on postsynaptic proteins, thus, insulin administration can increase NMDA receptor trafficking to postsynaptic membrane ${ }^{[27]}$, and stimulate tyrosine (Tyr) phosphorylation in NR2A and NR2B subunits ${ }^{[28]}$. Furthermore, in rat hippocampal slices, insulin stimulates the postsynaptic density protein 95 (PSD-95) translation to membrane, through the phosphatidylinositol 3-kinase PI3K-Akt-mammalian target of rapamycin (mTOR) signalling pathway ${ }^{[29]}$. Furthermore, insulin can also affect long-term depression (LTD) (see note ${ }^{2}$ ), via promoting the AMPA receptor endocytosis ${ }^{[30]}$. In addition to glutamatergic transmission, insulin can also modulate inhibitory synapses through type A $\gamma$-aminobutyric acid (GABA) receptor activity ${ }^{[31]}$.

Not surprisingly, reducing hippocampal IR expression disrupts the neuroplasticity leading to spatial memory deficits ${ }^{[32]}$. Furthermore, insulin inoculation in brain can reverse the alterations in spatial memory caused by inflammation, in lipopolysaccharides (LPS)-inoculated rats ${ }^{[33]}$ and in a chronic stress model ${ }^{[34]}$. Also in clinical studies, intranasal (IN) insulin administration for 4 months was shown to improved delayed memory and preserved general cognitive function in AD patients [35].

IR activity can also affect psychiatric symptoms (i.e. mood disorders) by the modulation of dopamine (DA) ${ }^{[36]}$ and serotonin (5-HT) signalling ${ }^{[37]}$, either via upregulation of the monoamine oxidase activity (enzyme that degrade monoamines) ${ }^{[38]}$ or by increasing dopamine transporter (DAT) mRNA expression ${ }^{[39]}$. Furthermore, animals suffering type 1 diabetes mellitus (T1DM) or T2DM showed depressive-like behaviour and insulin sensitizers administration can counteract these symptoms ${ }^{[40]}$.

Moreover, there is also insulin signalling in glial cells. Insulin stimulation in astrocytes promotes proliferation ${ }^{[41]}$ and glutamate receptor expression ${ }^{[42]}$, indicating that these are insulinsensitive cells. In rat HPC, intracerebroventricular (icv) insulin infusion induce an increase in Akt Ser473 phosphorylation in microglia cells ${ }^{[43]}$.

However, despite the role of insulin and the deleterious effects of brain insulin resistance, the excess of insulin, hyperinsulinemia is also toxic to neurons and glia cells. For that reason, it is crucial to maintain optimal insulin sensitivity, in other to prevent hyperinsulinemia.

The IR is a transmembrane Tyr kinase receptor which comprises two $\alpha$ and two $\beta$ subunits. The $\alpha$ subunit contains the extracellular hormone-binding site and the $\beta$ subunit has a transmembrane domain and the intracellular Tyr kinase domain. Each IR can bind up to two or three insulin molecules, the first one with higher affinity than the rest. Insulin has a negative feedback effect being its own signalling regulator. Thus, there is a ligand-induced internalization of the IR contributing to decrease insulin signalling ${ }^{[44,45]}$. Once insulin binds to its receptor, the $\beta$ subunit of each $\alpha \beta$ complex autophosphorylates in Tyr residues. This leads to the binding and phosphorylation of the insulin receptor substrates (IRS) ${ }^{[45]}$. In addition to Tyr phosphorylation sites (activation), the IRS can be phosphorylated in serine/threonine (Ser/Thr) residues, acting as inhibitory feedback mechanism ${ }^{[46]}$. The phosphorylation in Ser/Thr is induced by insulin in hyperinsulinemic conditions, through the IRS1 serine phosphorylation by mTOR [47,48], dyslipidemia and inflammation. The pro-inflammatory cytokines released by macrophages can activate serine kinases including the inhibitor of nuclear factor kappa-B kinase subunit beta (IKK $\beta$ ), c-Jun N-terminal kinase (JNK) or mTOR-ribosomal protein S6 kinase (mTOR-S6K) ${ }^{[4]]}$.

\footnotetext{
${ }^{1}$ LTP is a laboratory electrophysiological strategy where th stimulation of presynaptic neuron induces an increased and sustained response, recorded postsynaptically. It has been widely used to study the molecular mechanisms of memory formation. LTP requires glutamate neurotransmission through AMPA and NMDA glutamate receptors.

${ }^{2}$ LTD is another form of synaptic plasticity involved in memory formation and consolidation.
} 


\subsubsection{Insulin receptor substrates}

Up to date four different isoforms of IRS in mammals have been described: IRS1, IRS2, IRS3 and IRS4. The members of this family may differ in tissue expression, subcellular distribution, and their ability to involve different intracellular signalling cascades. Generally, the mechanism of action is identical to all of them, the IRS when phosphorylated in Tyr residues bind and activate Src homology 2 (SH2) domain containing proteins, e.g. PI3K, the $\mathrm{SH} 2$ protein tyrosine phosphatase (SHPTP-2). This binding initiates multiple downstream cascades responsible of insulin pleiotropic actions ${ }^{[49,50]}$. The most studied substrates are IRS1 and IRS2, which have similar function, but not always interchangeable. Meanwhile, IRS3 and IRS4 appear to have a redundant role in the insulin signalling cascade and some authors ensure that humans lack IRS3 gene, being only a pseudogene ${ }^{[51]}$. Although in cultured rat adipocytes, the overexpression of both substrates (IRS3 and IRS4) promotes an increase of the recruitment of GLUT4 to the cell surface ${ }^{[52]}$, the disruption of IRS3 and IRS4 genes in mice do not lead to any alteration of the growth or glucose homeostasis ${ }^{[53,54]}$.

The first insulin receptor substrate identified was the IRS1, thus its role in the insulin/IGF1 signalling cascade is well established, mostly in neoplastic growth ${ }^{[55,56]}$, cell metabolism, and proliferation ${ }^{[57]}$. In mice, deletion of IRS1 gene promotes growth retardation ${ }^{[58]}$ and insulin resistance ${ }^{[59]}$. These mice are smaller compared to control mice.

IRS2 was discovered to mediate IRS1/IGF1 signalling after IRS1 and it was first suggested to be an alternative to IRS1 and that both substrates were functionally interchangeable. The IRS2 shares structural similarities with IRS1, and the $43 \%$ of their amino acid sequences (Fig. 1.1) ${ }^{[49]}$. However, the amino acids sequence from 591 to 786 of the IRS2 is an exclusive region (kinase regulatory-loop binding domain -KRLB-) not found in IRS1 ${ }^{[60]}$. This unique domain is an important difference with IRS1, underlying signaling differences between them ${ }^{[61]}$. For example, both IRS1 and IRS2 activates PI3K but its p110 $\alpha$ subunit is preferentially regulated by IRS2 ${ }^{[62]}$, and IRS1 is associated with 3-phosphoinositide-dependent protein kinase-1 (PDK1) meanwhile IRS2 with PKC ${ }^{[63]}$. Moreover, in terms of subcellular localization, IRS2 is mainly localised in the cytosol whereas IRS1 can also be located into the nucleus ${ }^{[64-66]}$. Studies in IRS1 knockout mice have shown that IRS2 can compensate the IRS1 signalling deficiency in liver and pancreatic $\beta$ cells, but not in skeletal muscle, brown adipocytes or fibroblasts ${ }^{[67-70]}$. Thus, IRS2 and IRS1 interchangeable activity is tissue dependent.

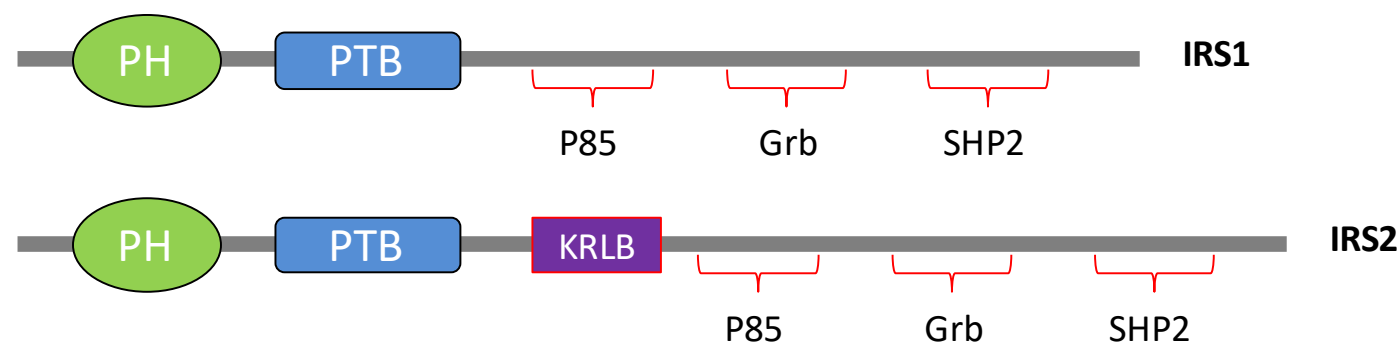

Fig. 1.1. IRS1 and IRS2 protein domains. Grb: growth factor receptor-binding protein; KRLB: kinase regulatory-loop binding domain; P85: regulatory subunit of phosphoinositide 3kinase; PH: pleckstrin homology; PTB: phosphotyrosine binding; SHP-2: Src homology domain (SH)-containing phosphatase-2.

IRS1 and IRS2 are distributed throughout the brain; HPC, Hyp, prefrontal cortex, nucleus accumbens, caudate, putamen, ventral tegmental area or cerebellum; but in all these areas the IRS2 mRNA expression has been found higher than IRS1 expression ${ }^{[71]}$. Unlike these, the IRS4 is 
mainly expressed in the Hyp, mostly in the arcuate nucleus and medial preoptic nucleus ${ }^{\text {[72] }}$ (Fig. 1.2). IRS4 in Hyp plays an important role in feeding and energy balance ${ }^{[73]}$, and the disruption of its gene is associated with central hypothyroidism ${ }^{[74]}$.

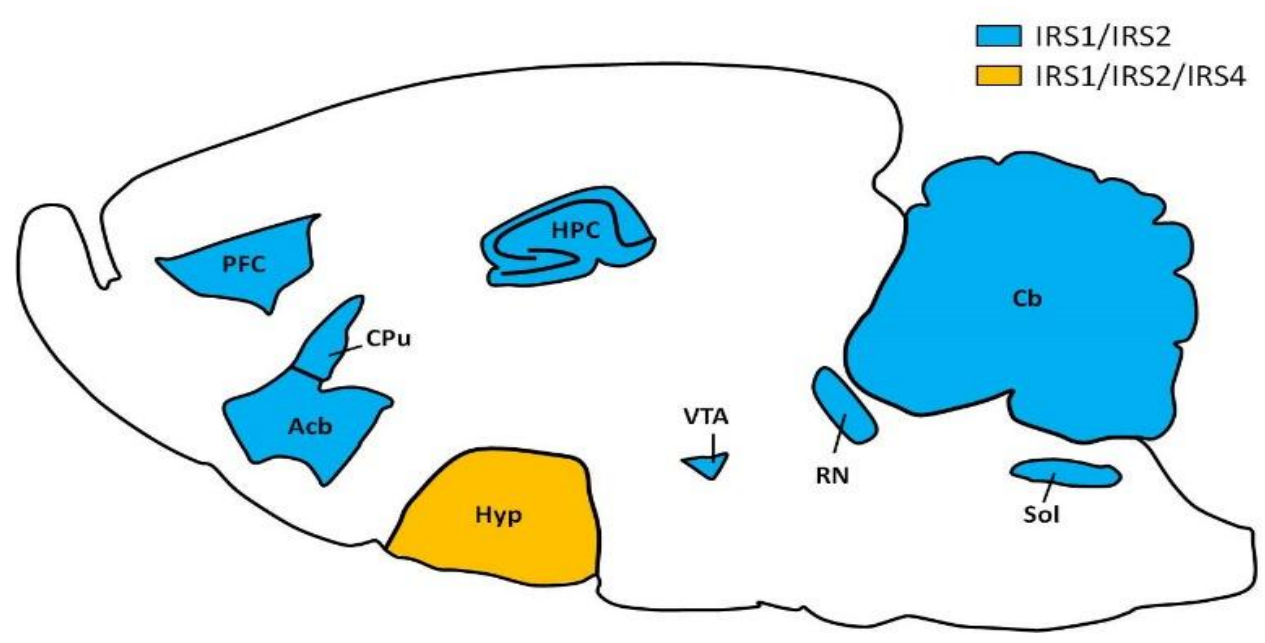

Fig. 1.2. Insulin receptor substrates (IRS) distribution throughout the mouse brain. Blue labelling indicates areas displaying IRS1 and IRS2 mRNA expression. Yellow labelling indicates areas displaying IRS1, IRS2 and IRS4 mRNA expression. Acb: nucleus accumbens; Cb: cerebellum; $\mathrm{CPu}$ : caudate putamen; HPC: hippocampus; Hyp: hypothalamus; PFC: prefrontal cortex; RN: raphe nucleus; Sol: solitary tract nucleus; VTA: ventral tegmental area. (Adapted from Kleinridders et al. 2014).

Alterations in IRS1 signalling affect brain function. In a post-mortem study of brains from patients affected by AD and tauopathies, IRS1 phosphorylation in 616 and 312 serines is increased compared to age-matched controls ${ }^{[75]}$. IRS1 pSer616 in the HPC of AD patients was strongly correlated with low episodic memory ${ }^{[76]}$. On the other hand, IRS1 pSer307 was found not associated to $\mathrm{AD}^{[77]}$, whereas altered IRS1 pSer312 has been confirmed in plasma exosomes from $\mathrm{AD}$ patients ${ }^{[78]}$. Furthermore, the Baltimore longitudinal study of predictive biomarkers of AD has shown that, together with Tau, higher levels of IRS1 pSer312 in plasma neuronal enriched vesicles are best predictive factors for AD ${ }^{[79]}$. Interestingly, IRS1 may also mediate other neurotrophic factors signalling by binding to their receptors, e.g. TrkB (Tropomyosin receptor kinase B): the brain-derived neurotrophic factor (BDNF) receptor ${ }^{[00]}$.

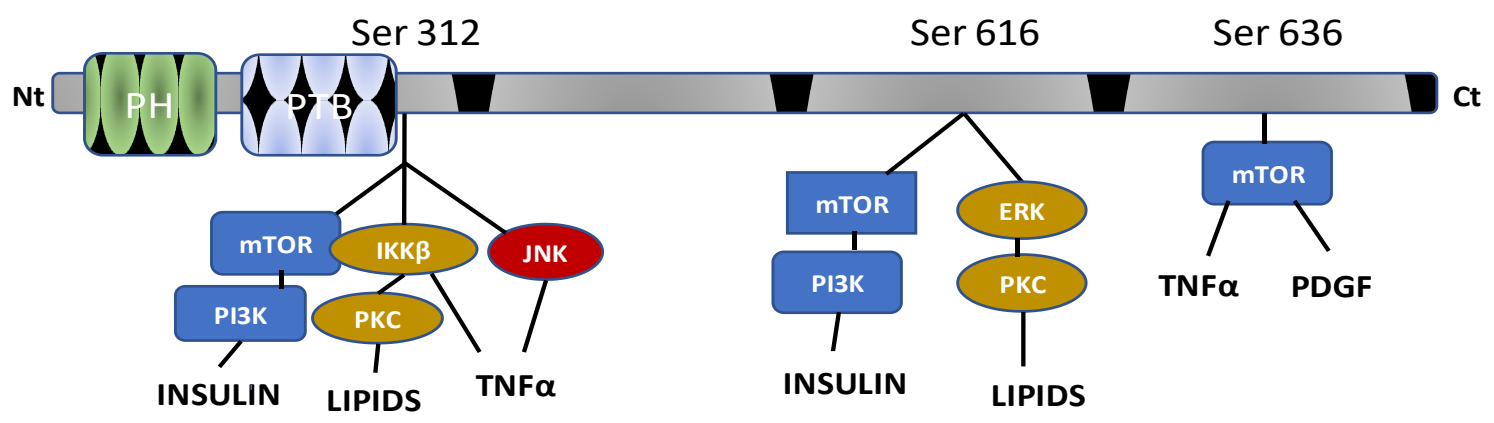

Fig. 1.3. Schematic representation of some serine phosphorylation sites in IRS1 and potential functions (in humans). The IRS1 phosphorylation in Ser312, Ser616 and Ser636 results in reduced insulin signalling (Adapted from Hançer et al. 2014). 
Mice that have IRS2 gene disruption display reduced brain growth but normal somatic growth. Specifically, there is a 50\% reduction of neuronal proliferation compared with wild type mice. Furthermore, knockout IRS2 mice show an increased Tau phosphorylation and an accumulation of Tau neurofibrillary tangles in the HPC ${ }^{[81]}$. IRS2 is involved in memory processes, since disruption of IRS2 gene blocks the activation of postsynaptic NMDA receptors in the HPC, thus impairing LTP $^{[82]}$.

All these data support the important role of insulin signalling for optimal brain function, showing that alterations on IR, IRS1 or IRS2 can cause feeding disorders, cognitive problems, and mood disorders. 


\subsection{Neuroinflammation and insulin resistance}

Upon insult, the organism inflammatory response mediated by pro-inflammatory markers release (cytokines and chemokines) counteracts the damage. This response is a necessary physiological process to heal the tissue. However, if this response remains, it turns into a chronic state where there is a hyperactivation of tissue-resident macrophages that release pro-inflammatory cytokines continuously, inducing further damage. In fact, chronic inflammation underlies different human diseases such as neurodegenerative diseases (e.g. AD), cardiovascular diseases (e.g. atherosclerosis), autoimmune diseases, diabetes, mental disorders, cancer and even accelerated aging ${ }^{[83]}$. Genetic and environmental events could increase the susceptibility to chronic

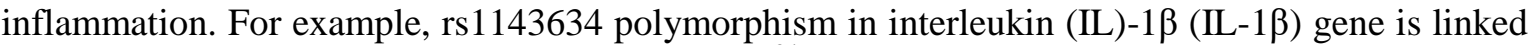
with periodontitis (teeth chronic inflammation) ${ }^{[84]}$. Also, stressful life experiences increase the expression of pro-inflammatory cytokines, as has been reported in people suffering grief associated to familiar loss ${ }^{[85]}$.

The chronic inflammatory response that occurs in the CNS is called neuroinflammation ${ }^{[86]}$. It is a process regulated by brain-resident macrophages, the microglia cells, required to recognize and eliminate any toxic component in the CNS ${ }^{[87]}$. Microglia has a high capacity for mobility, and they can switch between two different phenotypes, M1 (pro-inflammatory) and M2 (antiinflammatory), characterized by a different morphology and cytokine profile. The M2 phenotype is the "resting" type that actively monitors the brain in healthy conditions ${ }^{[88]}$. The switch to M1 begins with the recognition of the toxic insults that are classified into pathogen-associated molecular patterns (PAMPs) or the damage-associated molecular patterns (DAMPs). The receptors that recognized them are the pattern recognition receptors (PRRs). These include several families of receptors: the toll-like receptors (TLRs) both plasma and endosomal membrane; the cytoplasmic nucleotide-binding oligomerization domain (NOD)-like receptors (NLR); the intracellular retinoic acid-inducible gene-I-like receptors; and the transmembrane C-type lectin receptors ${ }^{[89]}$. PAMPs and DAMPs range from bacterial wall components (LPS) and virus capsid proteins, to debris released by dying cells and amyloid-beta $(\mathrm{A} \beta)$ oligomers ${ }^{[90]}$. The activation of this system by the so-called inflammasome, initiates the inflammatory cascade, which results in the secretion of several pro-inflammatory cytokines, such as tumor necrosis factor alpha (TNF $\alpha)$, interferon gamma (IFN- $\gamma$ ) and IL-1 $\beta$, IL-6 and IL-18. The pro-inflammatory cytokines purpose is to orchestrate the neutralization and elimination of the toxic molecules. In normal conditions, once the toxic stimuli have been cleared, microglia switch to the M2 phenotype and secretes anti-inflammatory cytokines such as IL-4, IL-10 and IL-18, BDNF or nerve growth factor (NGF), whose role is to terminate the innate immune response and contribute to restore the synaptic function. However, under pathological conditions, microglia cells do not go back to their resting state, thus causing a chronic inflammation process, with the overproduction of pro-inflammatory cytokines and reduction of neuroprotective factors that in sustained situations become highly toxic, leading to neurodegeneration ${ }^{[91]}$.

Chronic neuroimmune system activation underlies the initiation and progression in many dementias, such as $\mathrm{AD}^{\left[{ }^{[2-94]}\right.}$. In humans, increased levels of peripheral pro-inflammatory cytokines have been found in the serum of AD patients ${ }^{[95]}$. Not only A $\beta$ activates the microglia ${ }^{[96]}$, but also misfolded Tau interaction with microglia triggers inflammation ${ }^{[97]}$. The elimination of the microglial receptor NLR family pyrin domain containing 3 (NLRP3) has shown to reduce brain A $\beta$ levels in rodent models of $A{ }^{[98,99]}$; since then, the NLRP3 inflammasome has been deeply studied and characterized in $\mathrm{AD}^{[100,101]}$. In addition to the neurological symptoms, neuroinflammation also underlies the psychiatric signs associated with $\mathrm{AD}$, and for that reason, targeting neuroinflammation has also been proposed to treat those comorbid disturbances ${ }^{[102]}$.

Interestingly, different studies have shown that there is a lower incidence of $\mathrm{AD}$ amongst users of chronic non-steroidal anti-inflammatory drugs (NSAIDS) ${ }^{[103,104]}$, however NSAIDS administration do not counteract the cognitive decline once the disease is initiated ${ }^{[105,106]}$. Other anti-inflammatory drugs such as Etarnercept (specific TNF $\alpha$ inhibitor), did not show any improve in cognition, behaviour or global function in a clinical trial with mild to severe AD patients ${ }^{[107]}$. The failure of these treatments against inflammation to ameliorate AD symptoms indicates that 
there may be other different factors involved, including insulin resistance. Thus, treating just inflammation may not be sufficient to effectively prevent/treat the disease ${ }^{[108,109]}$.

Insulin metabolism alterations, especially the insulin resistance, are associated with metabolic and neurological diseases. Insulin resistance occurs when there is a reduced response to insulin, and it may be caused by different conditions, reduced levels of insulin/insulin-like growth factor-1 (IGF-1) receptor expression or binding and/or impaired IRS1/2 response. Peripherally, the principal role of insulin is to reduce blood glucose levels. Since centrally insulin is involved in many functions such as neuronal survival ${ }^{[110]}$, synaptic function and maintenance ${ }^{[111]}$, dendritic morphology and arbour development, energy homeostasis, learning and memory ${ }^{[112]}$ (Fig. 1.4).

Several situations can bring about insulin resistance: metabolic syndrome caused by high caloric diet, sedentarism, obesity; genetic predisposition and neuroinflammation ${ }^{[113]}$. In several models of insulin resistance, increased Tau phosphorylation, and amyloid-beta precursor protein expression(APP) expression together with higher levels of $\mathrm{A} \beta$ oligomers and deposition has been, found ${ }^{[114]}$. In addition, insulin resistance augments oxidative and ER stress, production of reactive oxygen species, mitochondrial dysfunction, and pro-inflammatory cascades ${ }^{[115]}$. Accordingly, T2DM is also an inflammatory condition since there is a sustained activation of macrophages and adipocytes with elevated pro-inflammatory cytokines release ${ }^{[116]}$. Since cytokines can cross the $\mathrm{BBB}$, the peripheral increment of circulating pro-inflammatory cytokines can activate CNS cytokines production. Inflammatory markers in the Hyp has been found in subjects with high consumption of dietary fats ${ }^{[117,118]}$. Conversely, central insulin resistance alters the systemic glucose regulation by Hyp ${ }^{[119]}$, worsening the metabolic syndrome.

Insulin resistance and inflammatory processes have been associated to classical aetiology processes of neurodegenerative diseases such as late-onset $\mathrm{AD}$. In fact, $\mathrm{AD}$ has been often recognized as Type 3 diabetes ${ }^{[120]}$. Post-mortem analysis of AD patients brains, defects in insulin and IGF-1 signalling have been reported ${ }^{[121-123]}$. Specifically, deregulation of IRS1 and IRS2 is associated to insulin growth factor-1 receptor (IGF-1R) resistance ${ }^{[124]}$.

For this reason, treatments targeting insulin resistance have been tested to fight $\mathrm{AD}$ progression. One of the strategies considered has been the administration of IN insulin. In a study using a streptozotocin-induced diabetic model, IN insulin restored learning and memory functions enhancing neurogenesis in HPC and olfactory bulb ${ }^{[125,126]}$. In a A $\beta$-inoculated rat model of AD, IN insulin reduced the level of tau hyperphosphorylation and microglial activation ${ }^{[127,128]}$. In humans, a systematic review on this strategy concluded that whereas IN insulin administration showed improvement in verbal memory and story recall, it was not effective on other aspects of cognition. Interestingly, the authors conclude that the treatment is affected by the apolipoprotein E (APOE) 4 isoform, where APOE $4(-)$ patients displayed more benefits compared to APOE $4(+)$ patients. This systematic review concluded that, although it is very safe (it does not interfere with systemic glucose levels), current data does not demonstrate that IN insulin can be used in humans as treatment for dementia of $\mathrm{AD}$ or mild cognitive impairment (MCI). Most importantly, these data supports that proper stratification by disease stage and APOE 4 carrier status must be considered to design better therapeutic strategies ${ }^{[129]}$. 


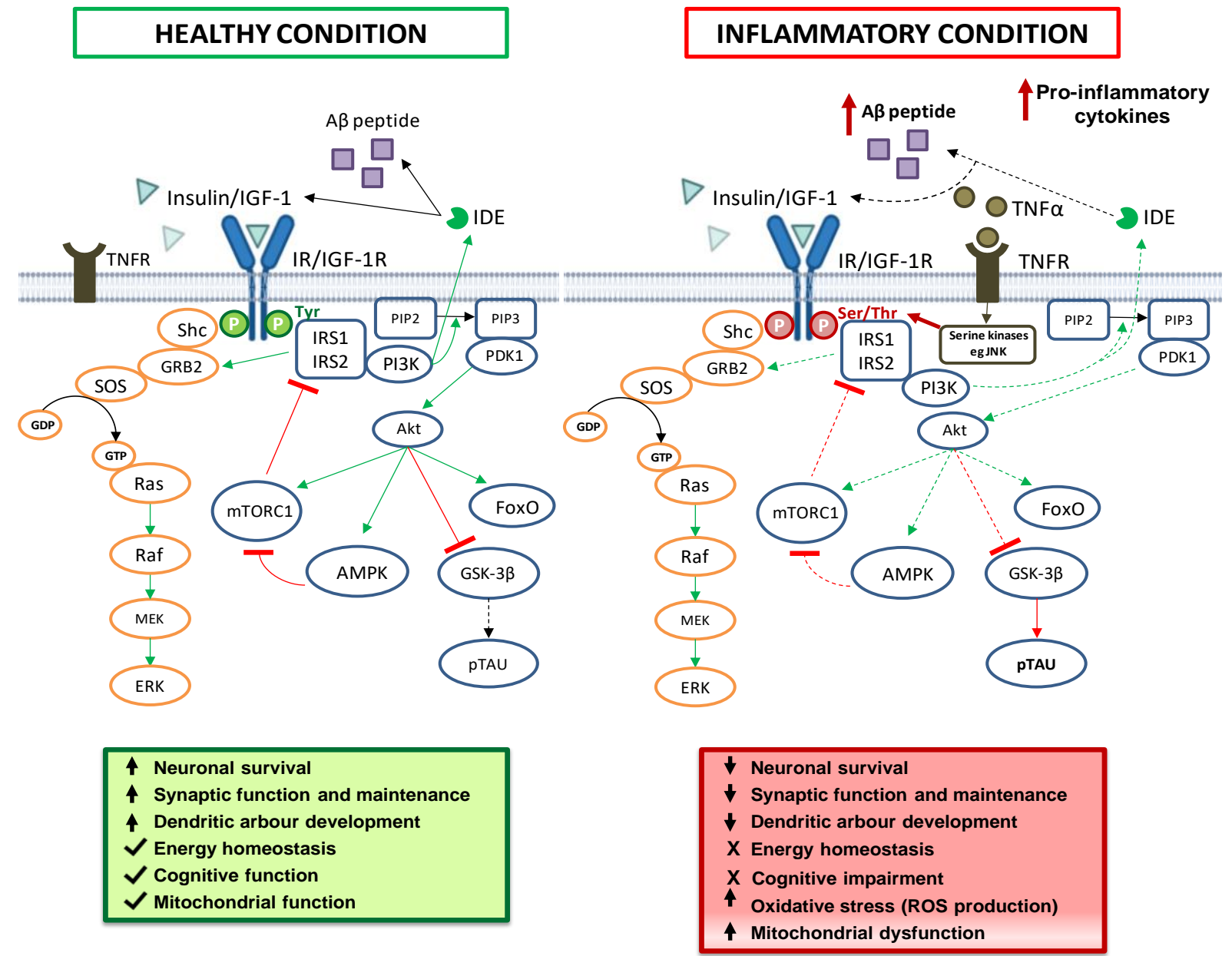

Fig. 1.4. Insulin signalling stimulates IRS1/2 tyrosine (Tyr) phosphorylation (healthy condition). Insulin resistance induced by IRS1/2 serine/threonine (Ser/Thr) phosphorylation (inflammatory condition). Upon ligand binding, recruitment of scaffolding proteins allows the stimulation of the Ras/ERK/1/2 and the PI3K/Akt pathways. In pathological situations, such as inflammation, insulin resistance reduces PI3K and Akt activity, leading to reduced IDE peptide degrading activity, and higher GSK-3 $\beta$ activity. AMPK: adenosine monophosphate-activated protein kinase; A : amyloid-beta peptide; ERK: extracellular signal-regulated kinase; FoxO: forkhead box O; GRB2: growth factor receptor-binding protein 2; GSK3 $\beta$ : glycogen synthase 3beta; IDE: insulin-degrading enzyme; IGF-1: insulin-like growth factor-1; IR/IGF-1R: insulin/IGF-1 receptor; IRS: insulin receptor substrate; JNK: c-Jun N-terminal kinase; MEK: mitogen-activated protein kinase; mTORC1: mammalian target of rapamycin complex 1; PDK1: 3phosphoinositide-dependent protein kinase 1; PI3K: phosphoinositide 3 kinase; PIP2: phosphatidylinositol 4,5-bisphosphate; PIP3: phosphatidylinositol (3,4,5)-trisphosphate; Ser/Thr: serine/threonine; Shc: src homology and collagen; SOS: son-of sevenless; pTAU: phosphorylated Tau; TNF : tumor necrosis factor alpha; TNFR: tumor necrosis factor receptor; Tyr: tyrosine (Adapted from Bedse et al. 2015).

In addition, since hyperinsulinemia, associated to insulin resistance (serum levels $>12$ $\mu \mathrm{IU} / \mathrm{mL}$ ) correlated positively with the severity of $\mathrm{AD}$ dementia ${ }^{[130]}$, other treatments designed to enhance insulin sensitivity, maybe a better choice of treatment, such as adenosine monophosphateactivated protein kinase (AMPK)- activators (e.g. Metformin ${ }^{[131]}$ ). AMPK improves insulin signalling by inhibiting mTORC1 activity, which performs a negative feedback mechanism over IRS1 and IRS2 (Fig. 1.4). Interestingly, AMPK activity also displays an anti-inflammatory effect, decreasing proliferation of inflammatory cells and their adhesion to the blood vessel endothelium. AMPK activity also reduces amyloidogenesis, Tau hyperphosphorylation, and the activation of autophagic degradation ${ }^{[132]}$. 


\subsection{Abscisic acid: insulin sensitizer and anti-inflammatory nutraceutical}

In 1963, two different laboratories specialized in plant biology described two substances: "dormin" involved in bud dormancy and growth inhibition ${ }^{[133]}$; and "abscisin II", involved in leaf abscission ${ }^{[134]}$. Few years later, "dormin" and "abscisin II" were found to be the same chemical molecule and it was renamed as "abscisic acid" (ABA) ${ }^{[135,136]}$. ABA is a phytohormone essential for the plant responses to biotic and abiotic stress situations (drought, extreme temperatures or high salinity) ${ }^{[137]}$.

Multiple ABA receptors have been identified in plants. One of these receptors is GCR2, a Gprotein coupled receptor (GPCR). Interestingly, GCR2 amino-acid sequence displays a high homology to the mammals lanthionine synthetase component $C$ (LANC) ${ }^{[138,139]}$. In rat pancreatic beta cells and human granulocytes, $\mathrm{ABA}$ binds to the lanthionine synthetase component $\mathrm{C}$-like protein 2 (LANCL2), a GPCR $\left(\mathrm{G}_{\mathrm{i}}\right)$ (pertussis toxin-sensitive) that activates cyclic adenosine monophosphate (cAMP) production, leading to intracellular calcium increase and cyclic adenosine difosfato-ribose (cADPR) generation ${ }^{[140]}$. In addition to LANCL2, ABA also interacts with the peroxisome proliferator-activated receptor $\gamma(\operatorname{PPAR} \gamma)$. This transcription factor is a nuclear receptor superfamily that support insulin responses in adipocytes ${ }^{[141]}$, and pancreatic beta cells ${ }^{[142]}$, likely through upregulation of GLUT4 ${ }^{[143]}$ and IRS2 expression ${ }^{[144]}$. The PPAR subfamily interacts with retinoid $\mathrm{X}$ receptors (RXRs) to create heterodimers. Interestingly, structural similarities, between $\mathrm{ABA}$ and retinoic acid (RA) have been identified ${ }^{[145]}$. In addition to direct binding, ABA may also activate PPAR $\gamma$ through cAMP/protein kinase A (PKA) signalling in macrophages and adipocyte tissue ${ }^{[146]}$, or via LANCL2 activation in monocytes ${ }^{[147]}$.

Interestingly, endogenous $\mathrm{ABA}$ production has been demonstrated in humans. Under elevated glucose concentrations pancreatic beta cells are activated and release ABA to stimulate insulin secretion using ADP as second messenger ${ }^{[148]}$, this signalling pathway has also been found in granulocytes ${ }^{[149]}$. Furthermore, the release of endogenous ABA in response to glucose, is impaired in T2DM patients ${ }^{[150]}$.

The insulin-sensitizing properties of ABA had been demonstrated in T2DM animal models, high fat diet (HFD) ${ }^{[118]}$ or $\mathrm{db} / \mathrm{db}$ mice ${ }^{[151]}$. In humans this phytohormone enhanced glucose tolerance in T2DM patients ${ }^{[152]}$ and improved the postprandial glucose homeostasis in healthy subjects ${ }^{[153]}$. The mechanisms underlying insulin-sensitizing properties could be via PPAR $\gamma$, similarly to other synthetic insulin sensitizer like the Thiazolidinediones (TZDs) family. Also via cAMP/PKA increase of glucagon-like peptide 1 (GLP1) release, which inhibits glucagon stimulation and raises insulin levels ${ }^{[154]}$, or by increasing glucose uptake by myocytes and adipocytes ${ }^{[155]}$.

In addition, $\mathrm{ABA}$ can regulate cell proliferation, however the results seem somewhat controversial, whereas in human mesenchymal stem cells ${ }^{[156]}$ and cultured hematopoietic progenitors ${ }^{[157]}$, ABA stimulates cell growth (via activation of $\mathrm{NF \kappa B}$, the nuclear factor kappalight-chain-enhancer of activated B cells); in glioblastoma cell culture, ABA induced apoptosis (via $\mathrm{RA}$ receptor $\alpha(\mathrm{RAR} \alpha)^{[158]}$. Surprisingly, in these glioblastoma cells, ABA reduced the PPAR $\gamma$ expression.

ABA can also regulate inflammatory mechanisms, but again, the results can be contradictory at least in cultured cells. On the one hand, ABA is pro-inflammatory, increasing monocyte chemoattractant protein-1 (MCP-1) levels to promote monocytes migration ${ }^{[159]}$. On the other hand, the activation of PPAR $\gamma$ can induce human monocytes into the M2 anti-inflammatory phenotype ${ }^{[160]}$. Nevertheless, in in vivo studies point to an anti-inflammatory effect of ABA, in high-fat diet (HFD) animals, ABA administration ameliorated peripheral inflammation, reducing TNF $\alpha$, adipocyte hypertrophy and macrophage infiltration in adipose tissue ${ }^{[151]}$. 
About ABA central effects, before the publications from our group, there were only two studies, one preliminary data showing enhanced spatial memory in young rats treated with ABA ${ }^{[161]}$, and the effect of ABA reducing corticotrophin-releasing hormone (CRH) in rat Hyp ${ }^{[162]}$.

After our firsts publications (presented in this thesis) our group has shown that ABA can improve memory and reduce neuroinflammation in a triple transgenic mice model ${ }^{[163]}$ and very recently, this effect has been replicated in a 5x transgenic model of $\mathrm{AD}^{[164]}$ and in a streptozotocin rat model ${ }^{[165]}$. Corroborating our initial studies and indicating that $\mathrm{ABA}$ may have a great therapeutic potential, suggesting an anti-inflammatory response, perhaps due to the insulin sensitizing properties.

Inflammation is a key factor in pain sensitization since it underlies pathologic pain conditions and promotes the development of hyperalgesia or allodynia (abnormal pain sensation to normal stimuli) ${ }^{[166]}$. Recent data have demonstrated ABA effects on pain. Intrathecal administration of $\mathrm{ABA}$ increased pain threshold in male rats and this anti-nociceptive effect was mediated by PKA activation ${ }^{[167]}$. Furthermore, ABA effects on pain sensitivity were completely abrogated when animals received intracerebroventricular naloxone (opioid receptors antagonist), thus the analgesic effect of the phytohormone could be carried out by opioid receptors activation [168]. 


\subsection{Brain networks: the memory circuitry}

The major brain area involved in memory function is the HPC. Its role in memory formation was published with H.M case, the first patient which hippocampal ablation was reported. Due to an accident, H.M. began to suffer continuous epileptic attacks and so, the HPC was removed surgically to ameliorate the epilepsy. The epileptic attacks decreased but memory problems emerged. Specifically, H.M. displayed amnesia of declarative/explicit memory, which is the inability to remember events. Inside this kind of memory, H.M. had also damaged the episodic memory, so he could not recall particular personal experiences ${ }^{[169]}$. In other words, the patient had retrograde amnesia, he could not remember personal events of the past, but also anterograde amnesia since he had impaired acquisition of new memories. However, the learning of motor skills, although unconscious, remained intact. Thus, the implicit memory function was not hurt after the hippocampal ablation. This was possible because there are more brain structures implicated. The surrounding cortical (e.g. perirhinal and entorhinal cortices) and non-cortical (e.g. septum, mammillary bodies) areas modulate hippocampal function and support different types of memory and processing.

Two cortical areas are particularly important for spatial memory: perirhinal and entorhinal cortices. Both areas send information to HPC, which is the responsible of the associative function by encoding information to a context ${ }^{[170]}$. The perirhinal cortex (PC) is involved in visual recognition memory, processing specific details of objects (recollection) and identifying the ones explored previously (familiarity) ${ }^{[171]}$. Specific parts of the hippocampal formation, the CA1 and subiculum, receive projections from PC, and CA1 send connections back to PC ${ }^{[172]}$ (Fig. 1.5). CA1-subiculum projections are critical for object-location learning and memory ${ }^{[173]}$.

The PC is also connected with entorhinal cortex (EC), an area involved in encoding spatial information. The medial part of EC (MEC) is responsible of spatial processing from an objective framework ${ }^{[174]}$, whereas the lateral part (LEC) represents the subjective/egocentric analysis of external items of the environment ${ }^{[175]}$. In this area, specifically in MEC, a characteristic type of neurons called the grid cells are located. The firing of these neurons creates a grid-like regular hexagonal pattern that cover all the space available for the animal, providing a metric for the spatial environment ${ }^{[176]}$. Grid cells relate to the place cells of the HPC. Place cells are CA1 pyramidal neurons that starts firing selectively when the animal is at one or few particular locations in the environment ${ }^{[177]}$. Place cells not only indicate the position of the animal in a context but also the locations that the animal has visited previously ${ }^{[178]}$, so they play an important role in spatial memory. EC is the major cortical input of the dentate gyrus (DG) of the HPC to process spatial learning and memory, through the perforant pathway (pfp, Fig. 1.5). 


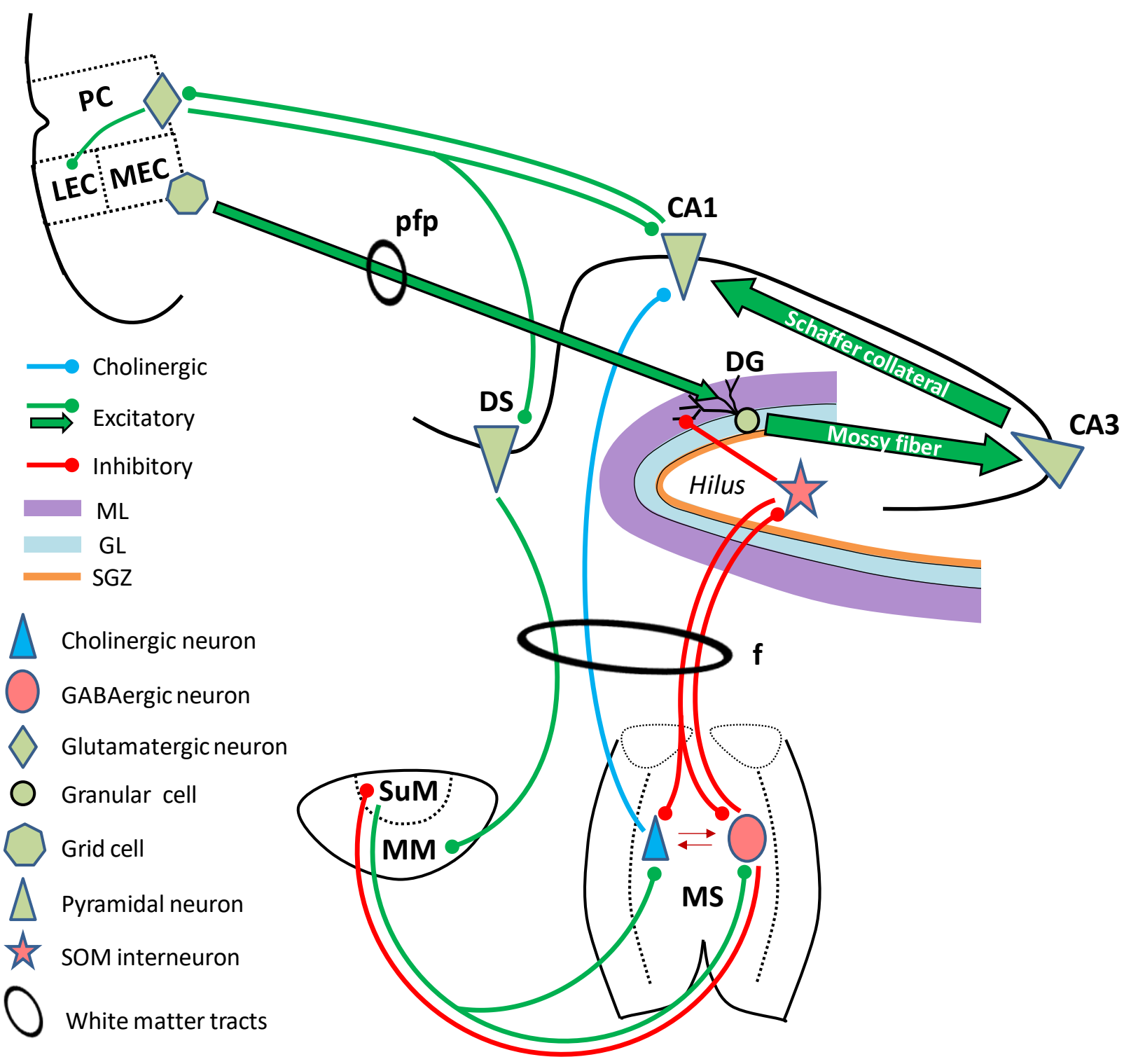

Fig. 1.5. Schematic hippocampal efferent/afferent connections.

Trisynaptic circuit: from the MEC (grid cells, green heptagon) to the granular cells in the DG, through perforant path, continuing to the CA3 pyramidal cells by the mossy fiber, and from these to CA1, via the Schaffer collateral. Regulation of this circuit by SOM interneurons (in the hilus, red star) is vital to determine CA1 firing pattern. Septo-hippocampal connection. Hilar interneurons send projections to GABAergic and cholinergic neurons in the MS through fornix. In turn, septal cholinergic and GABAergic neurons modulate CA1 and DG neurons, respectively. Papez circuit. The DS projects excitatory innervations to MM through the fornix, as part of the socalled Papez's circuit. Dorsal SuM sends glutamatergic projections and receives GABAergic feedback to and from the MS. In addition, the PC sends excitatory connections to the LEC, the DS and CA1, which in turn sends glutamatergic projections to PC. CA: Cornu Ammonis; DG: dentate gyrus; DS: dorsal subiculum; f: fornix; GL: granular layer; LEC: lateral entorhinal cortex; MEC: medial entorhinal cortex; ML: molecular layer; MM: mammillary bodies; MS: medial septum; PC: perirhinal cortex; pfp: perforant pathway; SGZ: subgranular zone; SOM: somatostatin interneuron; SuM: supramammillary nucleus (Adapted from Aimone et al. 2011, Khakpai et al. 2013, Vertes et al. 2001 and Yukie M. 2000).

The DG is a key point for generation of spatial memories. Information enters from EC to the granular layer (GL) of the DG. Granule cells are born by neurogenesis in the subgranular zone (SGZ) and communicate (via mossy fiber) to the CA3 pyramidal neurons, which, in turn, stimulate CA1 pyramidal cells (via Schaffer collateral) (green arrows in Fig. 1.5) ${ }^{[179]}$. This circuit is 
extremely relevant in the formation of new memories, and GABAergic neurons have been demonstrated to play a decisive modulating role. Amongst them, PV and somatostatin (SOM) positive neurons from the hilus of DG. SOM neurons regulate both nearby PV interneurons and, via long projections through fornix also the ChAT and PV neurons in the medial septum (MS) ${ }^{[180]}$ (Fig. 1.5). In turn, septal cholinergic and GABAergic neurons send ipsilateral projections through fornix to hippocampal formation to generate theta pattern, creating a regulatory feedback mechanism ${ }^{[181]}$ (Fig. 1.5). Not only septo-hippocampal connections are involved in the regulation of memory formation, the MS is in synchrony with the supramammillary nucleus (SuM, dorsal to mammillary bodies -MM-) neurons ${ }^{[182]}$ (Fig. 1.5).

In addition, the hippocampal formation is also an important part of the Papez's circuit: the major anatomical substrate of emotional control, mnemonic functions and episodic memories [183,184]. The information flows through different areas creating the following loop: subiculum of HPC $\rightarrow$ fornix $\rightarrow$ MM nucleus $\rightarrow$ anterior thalamic nucleus $\rightarrow$ cingulate cortex $\rightarrow$ parahippocampal gyrus (EC) $\rightarrow \mathrm{HPC}^{[185]}$.

In relation to human diseases like dementias, post-mortem studies of $\mathrm{AD}{ }^{[186,187]}$ and frontotemporal dementia ${ }^{[188]}$ patients revealed severe atrophy of anterior thalamus, MM and HPC (areas involved in Papez's circuit). Moreover, the cholinergic hypothesis, had been considered for a long time in the aetiology of $\mathrm{AD}$, as a consequence of septal cholinergic neurons degeneration [189]. Nowadays, this hypothesis is questioned as it cannot explain by itself the complex nature of the disease, however, cholinergic neurons death could be a consequence of a faulty circuitry (Fig. 1.5), for instance a lower inhibition from DG, since this would result in hyperexcitability. Supporting that idea, APOE 4 (the major genetic risk factor of late-onset AD) has been shown to induce hilar GABAergic alterations and subsequent memory dysfunction ${ }^{[190]}$. From that early report, in the last years, GABAergic cell therapy has been proposed as potential target for several neurological diseases ${ }^{[191]}$ included $\mathrm{AD}^{[192,193]}$. 
2

HYPOTHESIS \& OBJECTIVES 
Hypothesis 1: The administration of abscisic acid (ABA) improves neuroinflammation and cognitive effects induced by high fat diet through its modulation of insulin resistance and inflammatory response.

Objective 1: Confirm that $\mathrm{ABA}$ administration ameliorates peripheral high fat diet-induced insulin resistance.

Objective 2: Study the effect of ABA administration on the cognitive impairment induced by high fat diet.

Objective 3: Evaluate the impact of ABA administration over inflammatory markers in hypothalamus and hippocampus.

Objective 4: Study the effect of ABA administration on the expression of insulin receptor substrate 1 (IRS1) and substrate 2 (IRS2).

Objective 5: Confirm the impact of high fat diet in hippocampal neurogenesis and if $\mathrm{ABA}$ administration counteracts that effect.

Hypothesis 2: The specific downregulation of insulin receptor substrate 1 (IRS1) in neurons of the dorsal hippocampus induces cognitive impairment in the absence of neuroinflammation.

Objective 1: Determine the effect of shRNA IRS1 AAV-derived particle injection in dorsal hippocampus over anxiety, spatial memory, and depressive-like symptoms. connections.

Objective 2: Study the distribution of AAV-labelled dorsal hippocampus efferent

Objective 3: Characterize shRNA IRS1 AAV-infected neurons in dorsal hippocampus.

Objective 4: Study the impact of shRNA IRS1 AAV particle injection in dorsal hippocampus over synaptic plasticity in hippocampal-labelled projections.

Hypothesis 3: The administration of ABA ameliorates behavioural symptoms and altered pain sensitivity in a preclinical model of attention deficit hyperactivity disorder (ADHD).

Objective 1: Establish the animal model of ADHD: neonatal 6-hydroxydopamine (6OHDA)-induced lesion to confirm behavioural parameters.

Objective 2: Study the effect of ABA administration on the ADHD symptoms and pain sensitization developed by 6-OHDA-induced lesion. 


\section{3}

Chapter 1

THE EFFECT OF ABSCISIC ACID CHRONIC TREATMENT ON NEUROINFLAMMATORY MARKERS AND MEMORY IN A RAT MODEL OF HIGH-FAT DIET INDUCED NEUROINFLAMMATION 


\subsection{Introduction}

Obesity, a leading cause of type 2 diabetes ${ }^{[194]}$, correlates with cognitive impairment. Insulin sensitizers have been proposed as a promising tool for the reduction of obesity-induced insulin resistance and inflammation processes. The TZDs are a family of synthetic insulin sensitizer molecules; however, some of them have undesirable side effects ${ }^{[195-197]}$. Thus, alternative compounds with analogous properties but fewer side effects are needed. The phytohormone abscisic acid (ABA) was found in mammalian cells more than 25 years ago ${ }^{[198]}$. Since then, several studies have proposed it as a universal signaling molecule ${ }^{[199,200]}$. Structurally, ABA is very similar to the TZDs. Indeed, ABA can improve glucose tolerance ${ }^{[152]}$, reduce the level of TNF $\alpha$, and decrease adipocyte cell size in an in vivo model of obesity induced by HFD ${ }^{[201]}$. Moreover, in human and murine pancreatic cell lines (RIN-m and INS-2 cells), ABA can increase glucosestimulated insulin secretion ${ }^{[202]}$. This effect can be repressed using pertussis toxin and PKA inhibitors ${ }^{[148]}$. Dietary ABA also stimulates granulocyte function and macrophage infiltration in the adipose tissue ${ }^{[203]}$. In mammalian cells, the LANCL2 shows high homology with the ABA receptor in plants, the Arabidopsis GCR2. Silencing the expression of endogenous LANCL2 in granulocyte cells can abrogate ABA induction of $\mathrm{Ca}^{2+}$ responses, whereas overexpression of LANCL2 enhances the ABA-mediated effects ${ }^{[140]}$. Because of its role in the mediation of ABA effects, LANCL2 has been proposed as a therapeutic target for the treatment of inflammatory diseases and diabetes ${ }^{[204]}$.

Furthermore, ABA shows some molecular structural similarities to RA. The RA has beneficial cognitive effects, rectifying memory deficits in rodent models of AD. However, the clinical and animal model data show an association between RA administration and the symptoms of depression ${ }^{[205]}$. Chronic treatment with ABA has beneficial antidepressant effects demonstrated by increased sucrose intake, increased swimming in the forced swim test, and reduced expression of CRH and RAR $\alpha$ mRNA in the rat hypothalamus in control rats, with no reported side effects ${ }^{[162]}$. Moreover, preliminary data show an improved spatial memory in rats treated with ABA ${ }^{[161]}$. Based on these data, we hypothesized that as ABA can modulate peripheral insulin resistance and immune response, it might also exert similar action centrally. Thus, it this work aims to show whether dietary ABA could restore cognitive deficits resulting from a HFD-induced neuroinflammation. HFD elevates the levels of neuroinflammation markers in the brain ${ }^{[206]}$ and it might constitute a link between obesity and degenerative disorders via insulin resistance ${ }^{[207]}$. Furthermore, HFD has been shown to induce memory loss through elevation of inflammatory markers in HPC ${ }^{[208]}$.

We administered ABA $(20 \mathrm{mg} / \mathrm{L})$ in the drinking water to rats on either HFD or standard diet (SD). After eleven weeks of treatment, we compared the behavior of the four groups using two memory paradigms: the novel object recognition (NOR) and the T-maze. In addition, we measured $\mathrm{ABA}$ levels in the blood and cerebellum of all four groups using high-performance liquid chromatography (HPLC). We analyzed the microglia proliferation using immunohistochemistry. We demonstrated that the ABA administered in drinking water improved glucose tolerance and cognitive performance and decreased the levels of inflammatory markers in the hypothalamic areas. Our results confirm a therapeutic potential of this phytohormone in the peripheral metabolic alterations. The data also strongly suggest the potential beneficial effects of ABA in disorders of neuroinflammatory etiology, which has not been demonstrated before. 


\subsection{Methods}

The aim of the experiment is to evaluate the central effects of the phytohormone, $\mathrm{ABA}$ in a model of neuroinflammation elicited by HFD, during 12-week treatment. Behavioral tests started on the $11^{\text {th }}$ week, and sacrifices were carried out in the $12^{\text {th }}$ week of ABA and diet treatment (Fig. 3.1).

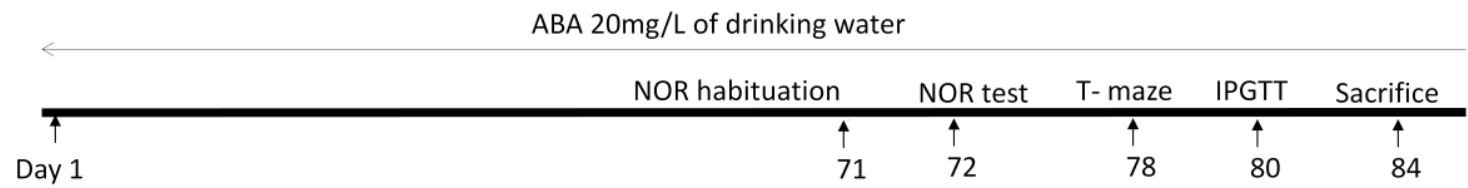

Fig. 3.1. Experiment design. Behavioral tests started in the $11^{\text {th }}$ week, and the animals were sacrificed in the $11^{\text {th }}$ and $12^{\text {th }}$ week.

\section{Animals and diet}

Eight-week-old male Wistar rats were obtained from the Janvier Labs (Saint-Berthevin, France) and kept at the animal facility of the University Jaume I. The procedures followed the directive 86/609/EEC of the European Community on the protection of animals used for experimental and other scientific purposes. The experiments were approved by the Ethics Committee of the University Jaume I. The animals were maintained on a $12 \mathrm{~h}: 12 \mathrm{~h}$ light-dark cycle and housed in pairs to reduce stress due to social isolation. Rats were divided randomly into four experimental groups: SD, control animals fed the standard rodent diet (Ssniff, Soest, Germany); SD-ABA, animals fed standard diet supplemented with ABA (Fernandez-Rapado, Spain) in the drinking water (20 mg/L); HFD, animals fed a high-fat diet (5736 kcal/kg, Ssniff) (Table 3.1); and HFD-ABA, animals fed high fat diet and ABA in drinking water $(20 \mathrm{mg} / \mathrm{L})$. We based our estimations in previous papers where ABA had been administered in the food pellet. Considering the daily food (20 g/day) and water average intake (50 mL/day), we estimated the amount of ABA per $\mathrm{mL}$ of drinking water, in order to doses in the same range as previous papers $(100 \mathrm{mg} / \mathrm{Kg})^{[151]}$. In Guri study, the period of ABA treatment was shorter (36 days) than our study (12 weeks), therefore we considered that the ABA administration could be lowered (1 mg/day) to achieve optimal chronic effects. The four groups were fed ad libitum for 12 weeks. The HFD induces inflammatory effects in the peripheral tissues ${ }^{[151]}$ and the brain ${ }^{[209]}$. The body weight and food and water intake were monitored twice a week per cage.

Table 3.1. Composition of high fat diet.

\begin{tabular}{lccc}
\hline Crude Nutrients & $\%$ & Additives & per kg \\
\hline Crude protein & 24.4 & Vitamin A (IU) & 15000 \\
Crude fat & 34.6 & Vitamin $\mathrm{D}_{3}(\mathrm{IU})$ & 1500 \\
Crude fibre & 6.0 & Vitamin E (mg) & 150 \\
Crude ash & 5.5 & Vitamin $\mathrm{K}_{3}(\mathrm{mg})$ & 20 \\
Starch & 0.1 & Vitamin C (mg) & 30 \\
Sugar & 9.4 & Copper (mg) & 12 \\
Fat & $60 \mathrm{~kJ} \%$ & & \\
Energy & $21.6 \mathrm{MJ}$ (or kcal) $\mathrm{ME} / \mathrm{kg}$ & & \\
\hline
\end{tabular}




\section{Novel object recognition (NOR)}

Training chamber consisted of a black wooden box $(81 \times 61 \times 52.3 \mathrm{~cm})$. Rats were habituated to the testing environment for $30 \mathrm{~min}$ on two consecutive days. On the first day, the rats spent a 10-min habituation period in the experimental box, without objects. On the second day, each rat was placed in the box for the familiarization phase and allowed to explore two identical objects (heavy metallic jars). One group (easy task group) was left for $10 \mathrm{~min}$, and the second group (difficult task group) was left to explore for $3 \mathrm{~min}$. All the rats were then returned to their home cages for a 1-h intertrial interval. The box and objects were cleaned with a $30 \%$ ethanol solution. In the test phase, the rats were returned to the box and allowed to explore a familiar and novel object for further 5 min (easy task group) or 3 min (difficult task group) ${ }^{[210]}$. Familiar and novel objects were alternated between left and right to prevent location predisposition. Both trial and test phases were recorded using a video tracking system (Smart 2.5.19, Panlab, Barcelona, Spain) for subsequent behavioral analysis. Exploration was defined as time spent sniffing within 1$4 \mathrm{~cm}$ of the object or touching it, always with the head oriented towards the object. Climbing over the object or running around it was not considered exploration.

\section{T-maze}

The T-maze was a three-arm maze; one arm $(119.3 \times 18.2 \mathrm{~cm})$ was longer than the other two, which were identical $(21.1 \times 34.5 \mathrm{~cm})$; the entire maze was placed above the floor. The longer arm was chosen as the start arm. The rats were habituated to the behavior room for $30 \mathrm{~min}$. On the test day, the animals were allowed to explore the maze for $5 \mathrm{~min}$, with access to two of the three arms (the home or start arm and the familiar arm). The rat was then returned to its home cage for a 2-h intertrial interval (difficult task group) or 90-min intertrial (easy task group), during which the maze was cleaned with $30 \%$ ethanol. The rat was then placed back in the maze; this time the animal had access to all arms for $5 \mathrm{~min}^{[211]}$. The number of entries to the novel arm and the time rat spent in each arm was recorded using a video tracking system (Smart 2.5.19).

\section{Glucose tolerance test}

Rats were fasted overnight, and a drop of blood was taken from the tail before $(\mathrm{t}=0)$ and 30 $\min (\mathrm{t}=30)$ and $120 \mathrm{~min}(\mathrm{t}=120)$ after glucose administration $(2 \mathrm{~g} / \mathrm{kg}$; IP -intraperitoneal-). Plasma glucose was measured using Glucomen LX Plus glucometer.

\section{HPLC ABA measurements}

ABA was analyzed in all four groups using LC/ESIMSMS, essentially as described in ${ }^{[212]}$, with slight modifications. Briefly, $1 \mathrm{~g}$ of frozen tissue was extracted in ultrapure water using a tissue homogenizer (Ultra-Turrax, Ika-Werke, Staufen, Germany) after spiking with $100 \mathrm{ng}$ of $\mathrm{d}_{6^{-}}$ ABA. After extraction and centrifugation, $\mathrm{pH}$ of the supernatant was adjusted to 3.0, and it was partitioned twice against diethyl-ether (Panreac, Barcelona, Spain). The organic layers were combined and evaporated in a centrifuge vacuum evaporator (Jouan, Saint-Herblain, France). The dry residue was then resuspended in water-methanol (9:1) solution, filtered and injected into a UPLC $^{\text {TM }}$ Acquity system (Waters, Milford, MA, USA). The analyte was then separated on a reversed phase UPLC C18 column (Nucleodur C18, $1.8 \mu \mathrm{m}, 50$ × $2.0 \mathrm{~mm}$, Macherey-Nagel, Barcelona, Spain). The solvents were methanol and water supplemented with $0.01 \%$ acetic acid, applied at a flow rate of $300 \mu \mathrm{L} \mathrm{min}{ }^{-1}$. ABA was quantified with a Quattro LC triple quadrupole mass spectrometer (Micromass, Waters, Manchester, UK) connected online to the output of the column through an orthogonal Z-spray electrospray ion source. Quantitation of this hormone was achieved by external calibration with known amounts of standards.

\section{Immunocytochemistry (ICC)}

Rats were anesthetized with an overdose of pentobarbital $(120 \mathrm{mg} / \mathrm{kg}$ Eutanax, Fatro, Barcelona, Spain) and transcardially perfused with saline $(0.9 \%)$ followed by $4 \%$ paraformaldehyde (PFA) fixative in $0.1 \mathrm{M}$ phosphate buffer, $\mathrm{pH}$ 7.4. After the perfusion, the brains were removed from the skulls and postfixed overnight at $4^{\circ} \mathrm{C}$ in PFA. Then, the brains were 
immersed in $30 \%$ sucrose for $48 \mathrm{~h}$ for cryoprotection. Sliding Microtome Leica SM2010R (Leica Microsystems, Heidelberg, Germany) was used to obtain 40- $\mu$ m-thick coronal frozen sections. The brains were cut in rostrocaudal direction; six series of slices were collected from each brain and stored at $-20^{\circ} \mathrm{C}$ for analysis.

For ionized calcium-binding adaptor molecule 1 (Iba1) staining, we used a goat anti-Iba1 (Abcam, Cambridge, UK). Briefly, free-floating sections were rinsed twice in $0.05 \mathrm{M}$ Tris-buffered saline (TBS, pH 8.0) and once in TBS with $0.2 \%$ Triton X-100, at room temperature (RT). Sections were incubated in $4 \%$ normal donkey serum for $1 \mathrm{~h}$ to reduce nonspecific labeling. Afterward, the sections were incubated in the primary antibody solution diluted 1:500 in $0.01 \mathrm{M}$ phosphate buffered saline (PBS) containing $2 \%$ normal donkey serum, TBS with $0.2 \%$ Triton X100 , and $2 \%$ bovine serum albumin for $24 \mathrm{~h}$ at RT. After washing off the excess of the primary antibody, the sections were incubated in biotinylated donkey anti-goat secondary antibody (Jackson) (1:200 in TBS, $0.2 \%$ Triton X-100). Two hours later, the sections were transferred to the avidin-biotin-horseradish peroxidase complex solution (Standard Elite ABC kit, Vector Laboratories Burlingame, CA USA) for $90 \mathrm{~min}$, followed by two rinses with TBS with $0.2 \%$ Triton $\mathrm{X}-100$, and two more with $0.05 \mathrm{M}$ Tris/ $\mathrm{HCl} \mathrm{pH} \mathrm{8.0.} \mathrm{Then,} \mathrm{the} \mathrm{sections} \mathrm{were} \mathrm{processed} \mathrm{in}$ $0.05 \mathrm{M}$ Tris/ $\mathrm{HCl} \mathrm{pH} 8.0$ containing $3.125 \mathrm{mg}$ of 3,3'-diaminobenzidine (DAB) and $2 \mu \mathrm{L}$ of $\mathrm{H}_{2} \mathrm{O}_{2}$ for 15-20 min. The reaction was stopped by adding an excess of $0.05 \mathrm{M}$ Tris/ $\mathrm{HCl} \mathrm{pH} \mathrm{8.0,} \mathrm{followed}$ by several rinses in PBS. Finally, the sections were mounted onto gelatin-coated slides, air-dried, dehydrated in alcohol, cleared in xylene, and coverslipped with dibutylphthalate polystyrene xylene (DPX) mounting medium.

\section{RNA extraction and RT-PCR}

Total RNA was extracted from the Hyp ( $\mathrm{n}=4-6$ per group) using RNeasy Lipid Tissue Mini Kit (Qiagen, Valencia, CA, USA) according to the manufacturer's protocol. The RNA samples were resuspended in $50 \mu \mathrm{L}$ of nuclease-free water. RNA concentration and quantification of total RNA was performed using Thermo Scientific NanoDrop 2000c, with the OD260/OD280. Genomic DNA was removed using DNase I, RNase-free (Life Technologies, USA), for $30 \mathrm{~min}$ at $37^{\circ} \mathrm{C}$. The reaction was stopped by addition of $1 \mu \mathrm{l}$ of EDTA for $10 \mathrm{~min}$ at $65^{\circ} \mathrm{C}$. The first strand cDNA was synthesized using the Prime-Script ${ }^{\mathrm{TM}}$ RT Reagent Kit (Perfect Real Time) (Takara Bio Inc., Shiga Japan). For each reaction, $1 \mu \mathrm{g}$ of RNA was used for reverse transcription, in a mixture of $4 \mu \mathrm{L}$ of 5x PrimeScript Buffer; $1 \mu \mathrm{l}$ of PrimeScript RT, $1 \mu \mathrm{L}$ of Oligo dT Primer $(50 \mu \mathrm{M})$, and $1 \mu \mathrm{L}$ of random primer $(100 \mu \mathrm{M})$. Enzyme mix was adjusted to a final volume of $20 \mu \mathrm{L}$ at RT. The mixture was incubated at $37^{\circ} \mathrm{C}$ for $15 \mathrm{~min}$ and heated at $85^{\circ} \mathrm{C}$ for $15 \mathrm{~min}$ to terminate the reaction. The cDNA was subsequently stored at $-20^{\circ} \mathrm{C}$. RT-PCR was performed in a volume of $10 \mu \mathrm{L}$ with $5 \mu \mathrm{L}$ of Maxima SYBR Green/ROX qPCR Master Mix (2X) (Applied Biosystems Life Technologies, Carlsbad, CA, USA), $1 \mu \mathrm{L}$ of primer and $1 \mu \mathrm{L}$ of cDNA. All PCR reactions were performed under the following conditions: initial cycle at $98^{\circ} \mathrm{C}$ for $10 \mathrm{~min}$ followed by 40 cycles at $98^{\circ} \mathrm{C}$ for $10 \mathrm{~s}$, $60^{\circ} \mathrm{C}$ for $10 \mathrm{~s}$, and $72^{\circ} \mathrm{C}$ for $20 \mathrm{~s}$. Gene expression in the Hyp and HPC was quantified using a StepOnePlus Real-Time PCR system (Applied Biosystems Life Technologies). The RT-PCR primers for TNFa were Forward 5'GACCCTCACACTCAGATCATCTTCT3' and reverse 5'TGCTACGACGTGGGCTACG3'. Each sample was tested in triplicate. Data were analyzed using the comparative critical threshold method, with the amount of target gene normalized to the housekeeping gene $\beta$-actin. Relative gene expression was calculated using $2-{ }^{\Delta \Delta \mathrm{Ct}}$ relative to control. 


\subsection{Results}

\subsubsection{ABA treatment improves glucose tolerance of HFD-fed animals}

Animals were weighed, and water and food consumption was monitored twice a week. Behavioral tests started on the $11^{\text {th }}$ week, and sacrifices were carried out in the $12^{\text {th }}$ week of ABA and diet treatment (Fig. 3.1). As expected, overnutrition affected the body weight, but ABA administration did not affect the weight gain in either group. The results are presented as the means \pm SE ( $n=16$ per group). Control animals fed SD increased their body weight from $444 \pm 11.7 \mathrm{~g}$ (week 1) to $585 \pm 12.0 \mathrm{~g}$ (week 10). Similarly, animals on SD supplemented with ABA increased their body weight from $446 \pm 10.7 \mathrm{~g}$ (week 1) to $593 \pm 10.2 \mathrm{~g}$ (week 10). This represents an increment of $132 \pm 2.4 \%$ and $133 \pm 1.5 \%$, for SD and SD with ABA, respectively. However, animals fed HFD and HFD-ABA increased their body weight from $448 \pm 11.7$ and $453 \pm 12.2 \mathrm{~g}$ (week 1) to $659 \pm 16.0$ and $669 \pm 16.2 \mathrm{~g}$ (week 10), an increase of $148 \pm 3.2 \%$ and $148 \pm 2.8 \%$, respectively (Fig. 3.2A). Data were analyzed using a two-way analysis of variance (ANOVA); time $\left(\mathrm{F}_{(10,600)}=994, \mathrm{p}<0.0001\right)$ and diet $\left(\mathrm{F}_{(3,60)}=6.08, \mathrm{p}=0.001\right)$ had a significant effect. Food and water consumption was measured per cage. Food intake data are represented by the mean $(\mathrm{g})$ of food consumed \pm SE per cage each week ( $\mathrm{n}=8$ per group). Weekly consumption was steady during the 12 weeks treatment, but the diet clearly affected food intake. The data were analyzed using two-way ANOVA $\left(\mathrm{F}_{(3,28)}=75.36, \mathrm{p}<0.0001\right)$. To determine the ABA intake, we monitored weekly water consumption (Fig. 3.2C). Based on this information, we calculated ABA intake for both HFD and SD fed animals, and confirmed that both groups had a similar average weekly intake of the hormone, $12.30 \pm 1.4$ and $13.27 \pm 0.8 \mathrm{mg} /$ week/cage, respectively (Fig. 3.2D).

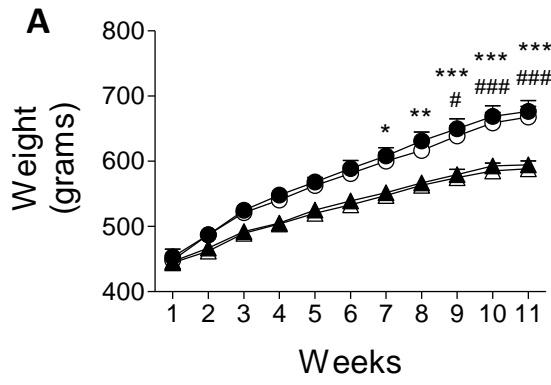

B

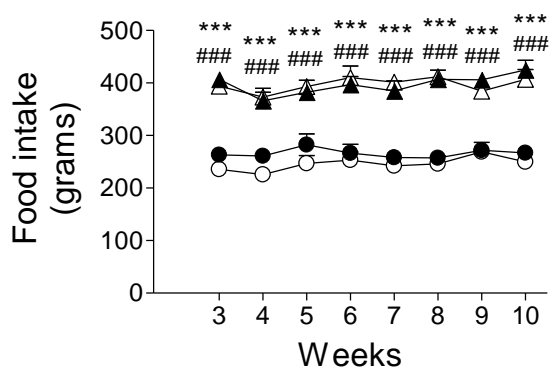

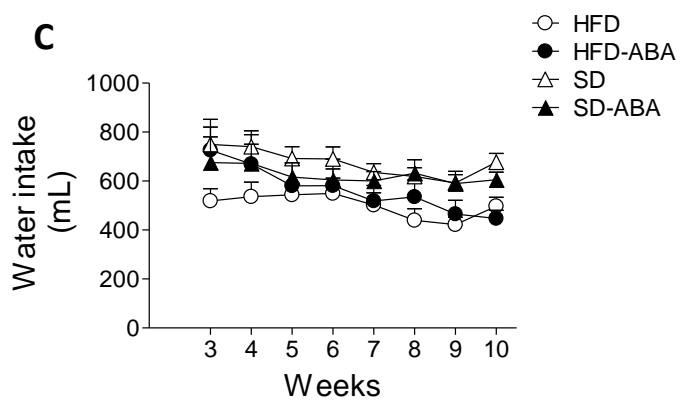

D

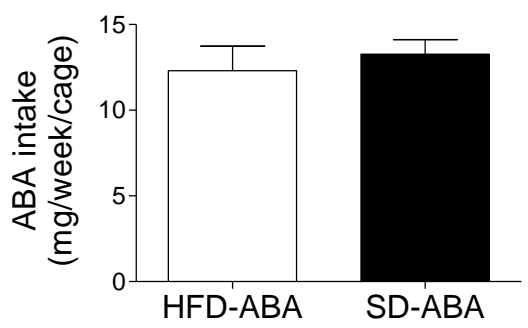

Fig. 3.2. Body weight in grams (A), food intake (B), water intake (C) and ABA weekly comsumption per cage (D) of rats fed high-fat diet (HFD, white circles); HFD with ABA (HFD$\mathrm{ABA}$, black circles); standard diet (SD, white triangles), and SD-ABA (black triangles) for 10-11 weeks; $\mathrm{n}=16$ per group. Values are mean \pm SEM. \#, $p<0.05$; \#\#, $p<0.01$; \#\#\#, $p<0.001$ for HFD vs. SD. $*, p<0.05 ; * *, p<0.01, * * *, p<0.001$ for HFD-ABA vs. SD.

It has been reported that dietary $\mathrm{ABA}$ given in the food pellets $(100 \mathrm{mg} / \mathrm{kg}$ food $)$ can improve glucose tolerance ${ }^{[151]}$. To confirm that, in our model, ABA $(20 \mathrm{mg} / \mathrm{L}$ of drinking water) improved the glucose homeostasis, we performed a glucose tolerance test by intraperitoneal injection of glucose $(2 \mathrm{mg} / \mathrm{kg})$ in fasted animals. After 11 weeks of treatment, the animals fasted overnight $(12-13 \mathrm{~h})$. The basal glucose levels were similar in all groups; $30 \mathrm{~min}$ after glucose injection, blood glucose levels increased. No significant differences were observed between the 
groups. However, two hours after injection, glucose levels in HFD group remained higher (235.4 \pm $36 \mathrm{mg} / \mathrm{dL})$ than in HFD-ABA group $(158.9 \pm 11.23 \mathrm{mg} / \mathrm{dL})$. In the latter group, the glucose levels were similar to those in control groups, SD $(143.9 \pm 10.24 \mathrm{mg} / \mathrm{dL})$ and SD-ABA $(152.6 \pm 15.52$ $\mathrm{mg} / \mathrm{dL}$ ). The data were analyzed using one-way ANOVA, with Newman-Keuls post-hoc test ( $\mathrm{p}<$ 0.05) (Fig. 3.3).

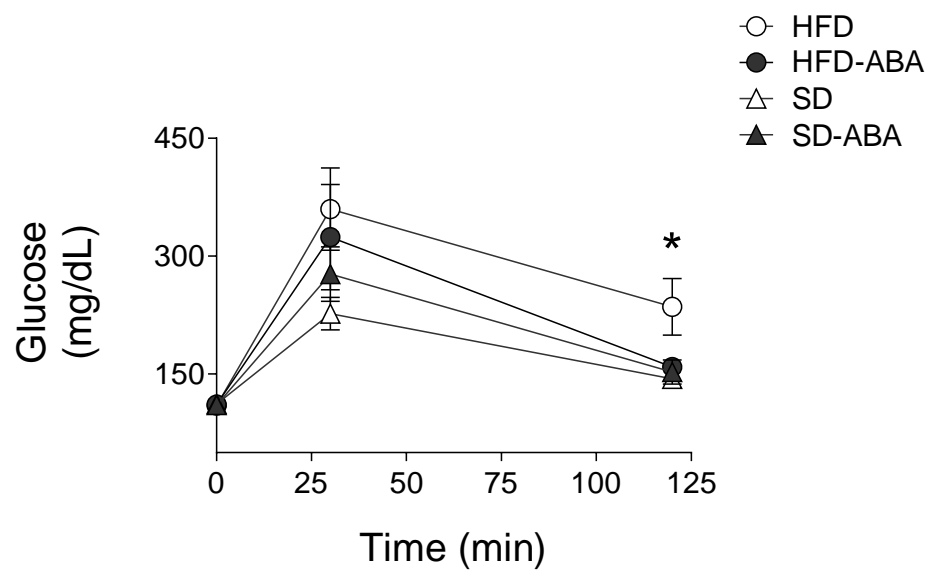

Fig. 3.3. The effect of dietary ABA (20 mg/L drinking water) on blood glucose levels in response to intraperitoneal (IP) glucose tolerance test. After 12 weeks of treatment, animals from the four groups were fasted overnight $(12-13 \mathrm{~h})$ and given $2 \mathrm{mg} / \mathrm{kg}$ of glucose by IP injection. Blood drops were taken from the tail, and plasma glucose concentration measured at the indicated times. Data are presented as the mean \pm SEM. White circles represent rats that fed HFD; black circles, HFD-ABA; white triangles, SD; and black triangles, SD-ABA. Data points at 120 min were analyzed using one-way ANOVA followed by post-hoc Newman-Keuls test. *, $p<0.05$ vs. HFD.

\subsubsection{ABA restores memory impairment induced by HFD}

HFD can induce memory impairments in rodents ${ }^{[213]}$ and humans ${ }^{[214]}$. To examine the effect of chronic ABA administration on cognitive performance, the animals were subjected to two behavioral paradigms that evaluate the memory in rodents, NOR and T-maze test. NOR exploits the innate exploratory preference of novel objects exhibited by rodents. This paradigm examines the capability of the animal to remember a familiar object when presented with a new one. We observed no significant differences in the time spent exploring the identical objects during familiarization phase (Fig. 3.4A and C). During the test, all four groups spent much more time exploring the novel object than the familiar one, and neither the diet nor ABA treatment changed these parameters (Fig. 3.4B). However, we observed differences when both the familiarization time and test time were reduced to $3 \mathrm{~min}$. In the HFD group, the times for familiar and novel object did not differ significantly, suggesting impairment in remembering the familiar object. HFD-ABA animals behaved in the same way as the control groups (SD and SD-ABA), indicating that ABA could abrogate the HFD-induced impairment (Fig. 3.4D). 
A

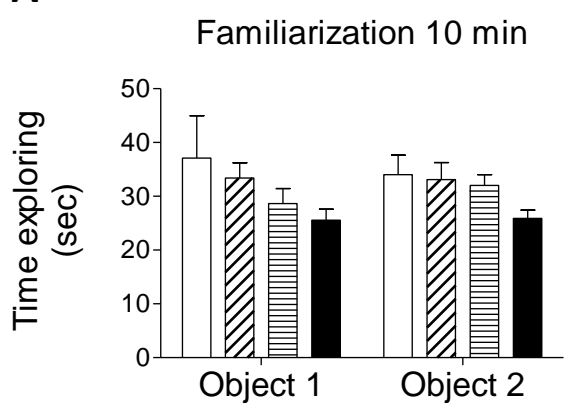

C

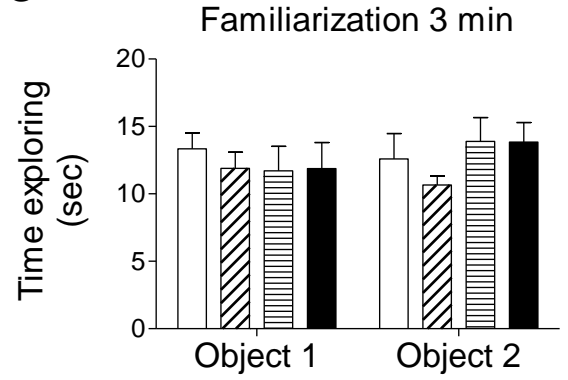

B Test $5 \mathrm{~min}$

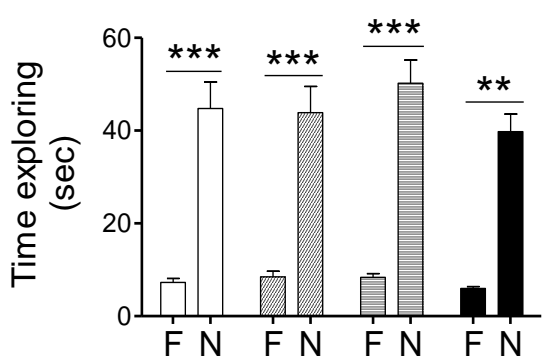

D

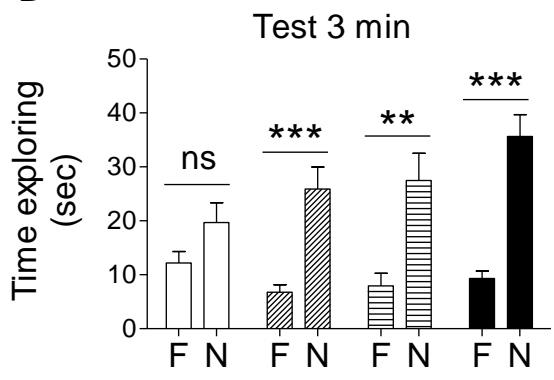

Fig. 3.4. Behavioral data for rats on a standard diet (horizontal lines, SD), high-fat diet (white columns, HFD), and supplemented with ABA in drinking water $(20 \mathrm{mg} / \mathrm{mL})$, HFD-ABA (diagonal lines) and SD-ABA (black columns) $(\mathrm{n}=8)$. Novel Object Recognition data: time exploring two similar objects during familiarization phase (A, C) and time exploring familiar and novel object during test phase $(B, D)$. Data are presented as mean \pm SEM and analyzed using paired Student $t-$ test, familiar vs. novel. **, $p<0.01$; ***, $p<0.001$; ns, non-significant.

In the T-maze test, we recorded the number of entries to both maze arms, one of which was familiar and the other was novel because it had been closed during the habituation. We observed that rats fed SD, SD-ABA, and HFD-ABA had a significantly higher number of entries to the novel arm than to the familiar one. This was not the case for the animals fed HFD only, suggesting that this diet might impair the memory of the familiar arm (Fig. 3.5A). The data were analyzed using unpaired Student's t-test, comparing familiar and novel arm; * $\mathrm{p}<0.05<* * \mathrm{p}<0.01$. Interestingly, when the test was performed with a longer inter-trial time between familiarization and test phases, which may be a more difficult working memory task, HFD-ABA did not rescue the alternation behavior shown by the HFD group. We found that the difference between the number of entries to the two arms was no longer significant for HFD-ABA animals, in similarity with HFD group. Both $\mathrm{SD}$ and SD-ABA group maintained a significant difference in the exploratory behavior, entering the novel arm more often (Fig. 3.5B). 
A

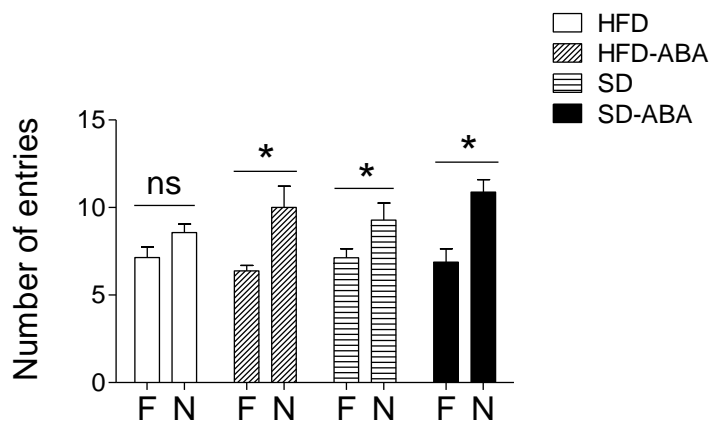

B

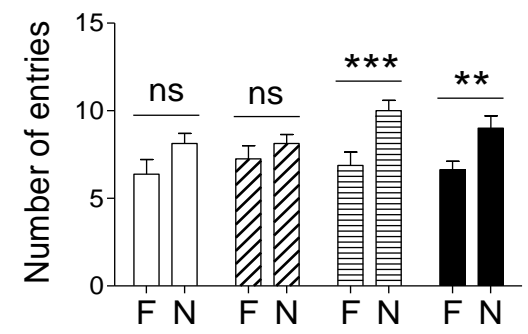

Fig. 3.5. Behavioral data for rats on a standard diet (horizontal lines, SD), high-fat diet (white columns, HFD), and supplemented with ABA in drinking water $(20 \mathrm{mg} / \mathrm{mL})$, HFD-ABA (diagonal lines) and SD-ABA (black columns) $(n=8)$. T-maze data: number of entries to novel and familiar arm after 90 min intertrial interval (A) and number of entries to novel and familiar arm after $2 \mathrm{~h}$ intertrial interval (B). Data are presented as mean \pm SEM $(n=8)$ and analyzed using paired Student t-test, familiar vs. novel. *, $p<0.05$; **, $p<0.01$; **, $p<0.001$; ns, non-significant.

\subsubsection{ABA administered in the drinking water can cross the $B B B$}

ABA levels in the blood and brain (cerebellum) were measured using HPLC. We detected variable amounts of circulating ABA in treated animals. However, we were unable to detect ABA under the same conditions in untreated animals, confirming that we, indeed, were observing exogenous $\mathrm{ABA}$ (Table 3.2). It has been reported that after intraperitoneal injections, $\mathrm{ABA}$ is widely distributed in the brain ${ }^{[161]}$. To ascertain that ABA crosses the BBB in our model, we measured the ABA levels in the cerebellum as a sentinel. In similarity with the serum examination, ABA was only detected in the brains of treated animals (Table 3.2). Interestingly, only a very small proportion of circulating $\mathrm{ABA}$ crosses the $\mathrm{BBB}$. Thus, we confirmed that our method of $\mathrm{ABA}$ administration in drinking water was appropriate for measuring potential peripheral effects; the compound could cross the BBB and might be responsible for the observed behavioral changes.

Table 3.2. ABA quantification by HPLC. Cerebellum (A) and blood (B) were extracted from all four groups of treated animals.

\begin{tabular}{llll}
\hline Cerebellum & & Serum & \\
\hline Diet group & ABA detected (ng/gr tissue) & Diet group & ABA detected (ng/gr tissue) \\
HFD & N.D. & HFD & N.D \\
HFD-ABA & $1.510 \pm 0.030$ & HFD-ABA & $196.8 \pm 82.57$ \\
SD & N.D. & SD & N.D. \\
SD-ABA & $2.980 \pm 0.100$ & SD-ABA & $47.95 \pm 13.80$ \\
\hline
\end{tabular}

N.D.: not detected

ABA was only detected in animals that had received exogenous administration of ABA in the drinking water $(20 \mathrm{mg} / \mathrm{L})$ 
3.3.4. HFD intake increases $T N F \alpha$ and reactive microglia levels in hypothalamus. $A B A$ can effectively counteract these changes

Next, we measured the levels of inflammatory markers in the brain. We used immunohistochemistry to detect the microglia-specific cytoplasmic marker Iba1 ${ }^{[215]}$ in the Hyp (Fig. 3.6A). We observed that the number of microglial cells increased significantly in rat Hyp of HFD-fed rats $(1613 \pm 260.5$ cells $/ \mathrm{mm} 2)$ in comparison with SD-fed controls $(754 \pm 135.2$ cells $/ \mathrm{mm} 2)$. The ABA treatment reduced this effect in HFD animals $(888 \pm 158.8$ cells $/ \mathrm{mm} 2)$ but did not affect SD-fed animals (794 \pm 178.4 cells $/ \mathrm{mm} 2$ ) (Fig. 3.6B; one-way ANOVA, $\mathrm{p}=0.0135$ ). Representative images of microglia in the Hyp are shown in Fig. 3.6 (HFD, Fig. 3.6C; HFD-ABA, Fig. 3.6D; SD, Fig. 3.6E; and SD-ABA, Fig. 3.6F). In HFD group, apart from the increase in the number of Iba1-positive cells, the microglia cells were less ramified and more amoeboid than in controls, indicating an activated state (insets in images). On the other hand, we found no significant differences in HPC, under the same conditions in all four groups (data not shown).

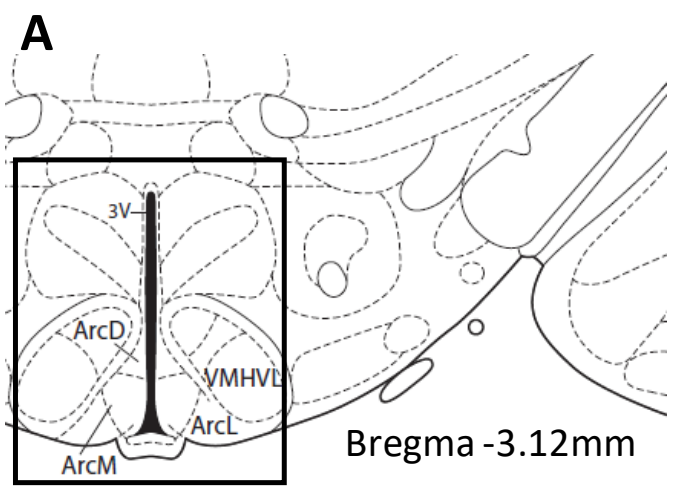

B
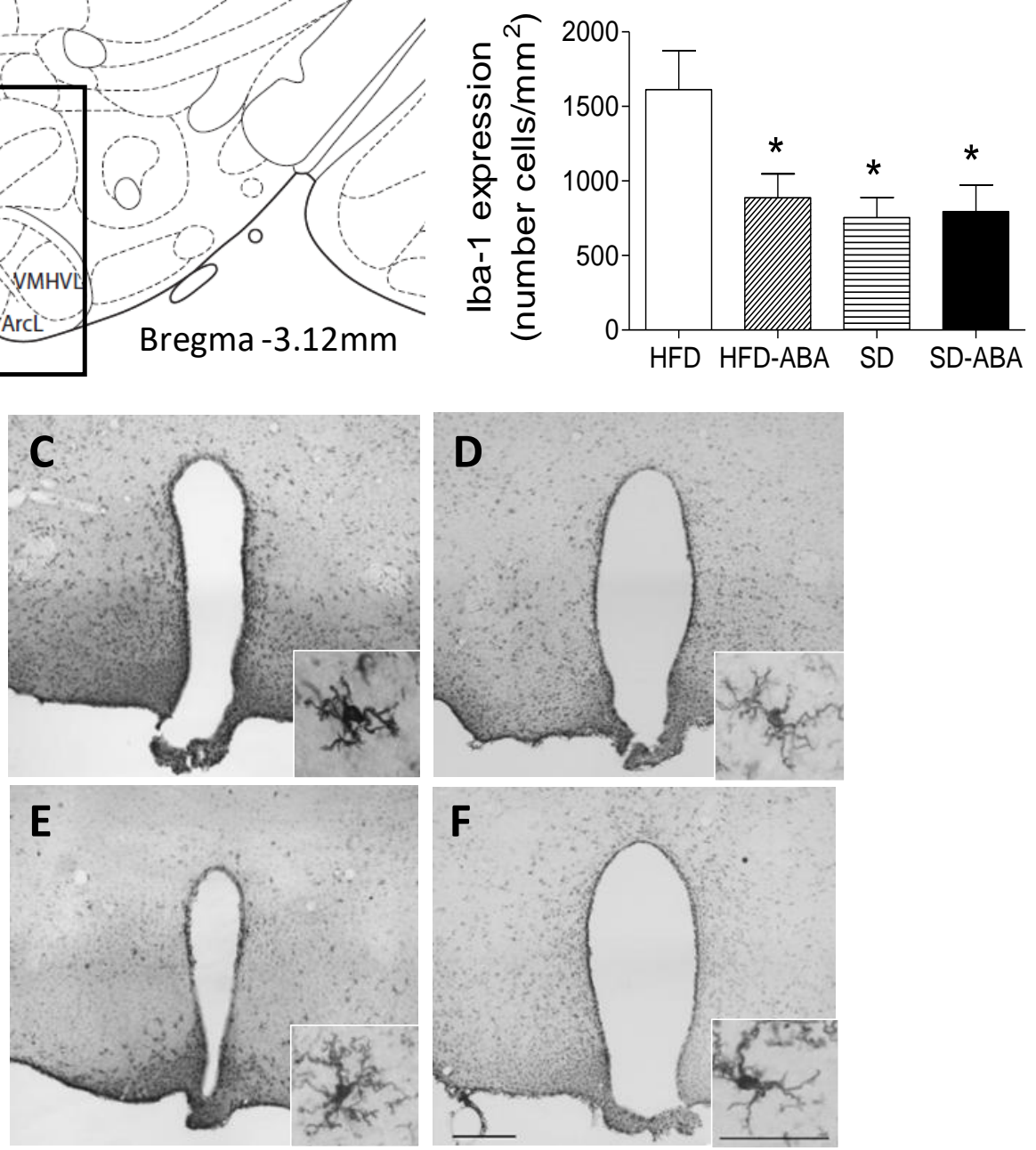

Fig. 3.6. Within the hypothalamus (Hyp), ABA curtails the increase in microglia staining induced by high-fat diet (HFD). Map showing the location of the analyzed hypothalamus (A). Anterior (bregma $-1.80 \mathrm{~mm}$ ) and medial (bregma $-3.12 \mathrm{~mm}$ ) hypothalamus were used for quantification (B). HFD fed rats (C); HFD-ABA (D); standard diet, SD (E); and SD-ABA (F). Calibration bar, $200 \mu \mathrm{m}(\mathrm{C}-\mathrm{F})$. Data are represented as the mean \pm SEM of density of cells per $\mathrm{mm} 2(\mathrm{n}=6)$. Data were analyzed using one-way ANOVA followed by post hoc Newman-Keuls test; ${ }^{*}, p<0.05$ vs. HFD. The reactive microglia are shown in the insets at high magnification. Calibration bar, $50 \mu \mathrm{m}$. 
Next, we examined the levels of inflammatory and anti-inflammatory cytokines in the Hyp. The Hyp was dissected from all groups, and total RNA was isolated. Quantitative PCR was carried out to determine the expression levels of different cytokines. We observed that TNFa levels increased in the Hyp of HFD-fed rats in comparison with controls, confirming the existing reports [216,217]. ABA administration restored TNF $\alpha$ levels to control values (Fig. 3.7).

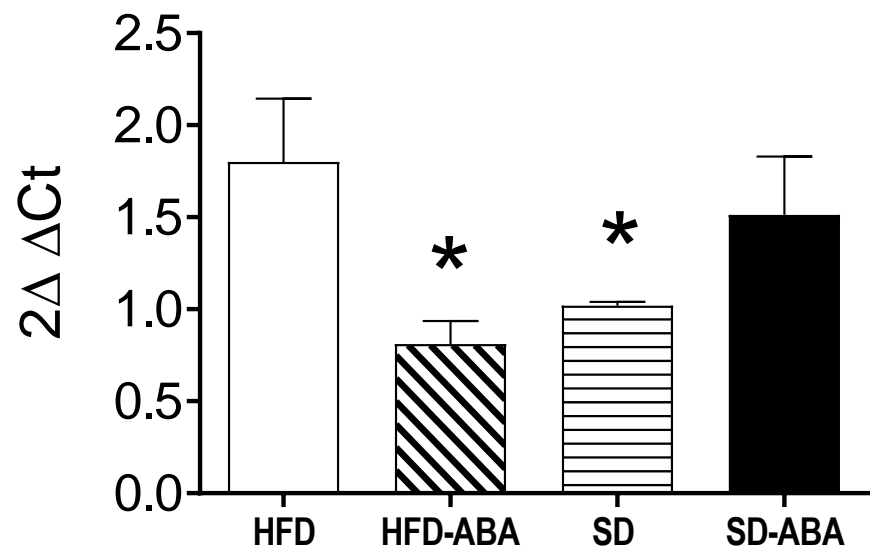

Fig. 3.7. Cytokine mRNAs levels in the hypothalamus: pro-inflammatory cytokine $\mathrm{TNF} \alpha$ in rats fed high fat diet (HFD, white columns); HFD supplemented with ABA in drinking water (20 $\mathrm{mg} / \mathrm{L}$ ) (HFD-ABA, diagonal lines); standard diet (SD, horizontal lines); and SD supplemented with ABA (SD-ABA, black columns). Data are presented as mean \pm SEM ( $n=4-6$ rats) and analyzed using one-way ANOVA followed by Newman-Keuls Multiple Comparison Test; *, $p<0.05$ vs. HFD. 


\subsection{Discussion}

Excessive energy intake is a major cause of obesity, which is recognized as one of the greatest threats to human health in the Western societies. Malfunction of hypothalamic neurons appears to underlie the pathology of metabolic diseases ${ }^{[218]}$. Even though the relationship between obesity (excess body fat) and neurodegeneration is highly complex, many sources link obesity and high fat consumption with certain neurodegenerative processes [207]. Growing evidence demonstrates that HFD induces insulin resistance, immunological and synaptic alterations in different brain areas ${ }^{[219,220]}$, and cognitive impairment ${ }^{[221]}$.

In animal obesity models, the phytohormone ABA, related structurally to insulin sensitizers (e.g. TZDs) and memory-improvement molecules (such as RA), ameliorates the symptoms of type 2 diabetes. It targets PPAR $\gamma$ in a manner similar to the TZDs class of anti-diabetic drugs ${ }^{[151,222]}$. In this study, we demonstrate, for the first time, that ABA can counteract some of the neurological effects (cognitive impairment and neuroinflammation markers) induced by HFD. We, in this study, and others have found that $\mathrm{ABA}$ can cross the $\mathrm{BBB}$, but whether the improvement on cognitive functions is caused by direct action of $\mathrm{ABA}$ in brain, remains elusive.

Our protocol was designed to avoid an increase in behavioral anxiety. ABA was administered in the drinking water $(20 \mathrm{mg} / \mathrm{L})$ and not in food pellets since rats fed on an HFD tend to eat less than controls [223]. The dose was based on previous studies reporting dietary administration of $\mathrm{ABA}{ }^{[151]}$. The vehicle used to dissolve ABA was added to water for SD and HFD groups to match any possible taste alterations. Some studies have used intraperitoneal injections (IP), which guarantee exact dosing but are highly anxiogenic. Another innovation in our study was housing the animals in pairs to reduce stress due to isolation ${ }^{[224]}$. We considered these issues important since stress can affect feeding and cognitive behavior in many indirect ways ${ }^{[225]}$. Therefore, reducing the stress levels (by avoiding isolation and daily injections) was desirable. To examine the intake of $\mathrm{ABA}$, we examined the average weekly water consumption in groups SDABA and HFD-ABA. Daily ABA intake was approximately $1.5-1.8 \mathrm{mg} / \mathrm{kg}$. During the 12 -week period, the intake average was uniform in all groups. To validate our strategy as an effective way of drug administration, we carried out a glucose tolerance test in all four groups. We confirmed that ABA improves glucose tolerance, reducing blood glucose levels to the normoglycemic range two hours after glucose injection.

The effect of specific diets on cognitive performance in rodents has been investigated using different behavioral paradigms. Diets with increased sugar and fat content cause impairment in spatial and working memory ${ }^{[226-228]}$ in both rats and mice. The link between obesity and inflammation of central nervous system is widely accepted; the Hyp is the area where inflammatory markers are most evident. Neuroinflammation underlies cognitive impairment ${ }^{[194]}$. On the other hand, some reports have suggested that ABA improves spatial memory ${ }^{[162]}$. ABA has antiinflammatory and insulin-sensitizing properties, and is a PPAR $\gamma$ agonist ${ }^{[229,230]}$. Some reports have shown that PPAR $\gamma$ agonism in the central nervous system ameliorates cognitive function ${ }^{[231,232]}$. Therefore, we hypothesized that ABA would alleviate the HFD-induced neuroinflammation and cognitive defects. To follow up this assumption, we examined the performance of the rats in two simple memory tests, NOR and T-maze. In contrast to other reports, ABA did not significantly affect the animals fed a control diet. This discrepancy might have been caused by differences in animal handling. As mentioned above, we housed the animals in pairs to reduce the isolation anxiety and ABA was administered in the drinking water, reducing the IP injection-induced stress. Under these conditions, the reduced anxiety levels might have a positive effect on test performance. In the studies showing antidepressant-like effects of $\mathrm{ABA}$, the compound has been administered daily using an IP injection ${ }^{[161]}$. We believe that in our model, the effect of ABA is independent of its antidepressant activity.

In a NOR working-memory test, all groups, including the HFD group, were able to discriminate between the novel and the familiar object. However, when the time spent in familiarization and test phases was shortened, thus reducing/hurting the acquisition phase, HFD-fed animals spent similar time exploring the novel and the familiar object. This indicated that they no 
longer remembered the familiar object. This behavior was efficiently counteracted by ABA treatment. ABA-treated animals behaved like the members of other groups, spending significantly more time exploring the novel object.

The alternation test (the T-maze test) is considered a reliable test to evaluate spatial working memory in rodents ${ }^{[233,234]}$. Using this paradigm, we found that HFD could impair alternation; the animals had difficulties in remembering the previously visited arms. Chronic treatment with ABA can restore this capability. However, when the intervals between the tests were increased, making the task harder, HFD-ABA-fed animals no longer alternated, behaving like the animals in the HFD group. This indicated that they had lost the ability to discriminate between the familiar and novel arms. We would not attribute these differences to ABA metabolism; not only ABA is administered chronically, but during the inter-trial interval rats have access to water and food. The peripheral anti-inflammatory effects ABA are well known. However, to the best of our knowledge, there is no data on the effect of $\mathrm{ABA}$ on the cerebral immune response in vivo. Moreover, in vitro data are controversial; some studies show that ABA activates the microglia ${ }^{[235]}$, whereas others report no effect ${ }^{[236]}$. Among the different types of glia, the resident macrophages in the brain, microglia, are responsible for cell debris clearance and release of cytokines that recruit other immune-responsive cells to the central nervous system ${ }^{[237]}$. In the metabolic syndrome induced by HFD, microglia in the Hyp adopts a pro-inflammatory state, which is linked to an abnormal increase in the production of pro-inflammatory cytokines. These might be toxic to the neurons ${ }^{[238,239]}$. In this study, we investigated the microglial expression in the Hyp in the four study groups. We examined the immuno-reactivity of Iba1, a cell marker upregulated during microglial activation ${ }^{[215]}$. In contrast to other studies of ABA effect on microglia in vitro, reporting either activation ${ }^{[233]}$ or no effect ${ }^{[236]}$, we demonstrated that in vivo, ABA reduces the number of Iba1-positive cells. This finding suggests an important practical application; the microglia hyperactivation has been found in a number of alterations including traumatic brain injury ${ }^{[240]}$, cerebrovascular accidents ${ }^{[241]}$, neurodegenerative disorders ${ }^{[242,243]}$, epilepsy ${ }^{[244]}$, schizophrenia ${ }^{[245]}$, and depression ${ }^{[246]}$. In addition, we analyzed Iba1 expression in dorsal HPC, but HFD did not increase microglia proliferation compared to control groups (data not shown).

Further to test the anti-inflammatory effects of $\mathrm{ABA}$ in the central nervous system, we measured the hypothalamic and hippocampal cytokine levels. The activated microglia synthesize and secrete pro-inflammatory cytokines, such as TNF $\alpha{ }^{[247]}$. We observed an increase in TNF $\alpha$ levels in the Hyp of HFD-fed rats. ABA treatment rescued this effect, lowering TNF $\alpha$ to control levels.

Importantly, in control rats, ABA treatment may worsen some parameters, that even not statistically significant, could point to a therapeutic window where ABA can restore deficits induced by HFD inflammatory processes, but it may not be advisable if there are not previous deficits. Further studies are required to elucidate the range for optimal ABA action.

In summary, we found that chronic treatment with ABA reduced HFD-induced microglia activation in Hyp, as revealed by Iba-1 staining. Also, TNF $\alpha$ levels were altered in Hyp of HFD-fed animals, and these changes were counteracted by ABA administration. Moreover, ABA ameliorated the HFD-induced impairments in cognitive performance. We found no changes in reactive microglia density in HPC. In this study, we show that hypothalamic inflammation can correlate with mild alterations in cognitive performance. Finally, based on our results, we conclude that $\mathrm{ABA}$ has a protective effect in neuroinflammation conditions, lowering microglia and TNF $\alpha$ levels and restoring cognitive function. Given the fact that ABA can cross the BBB; we postulate that this molecule could have a potential therapeutic value in the treatment of diseases of neuroinflammatory etiology. 
4

Chapter 2

ABSCISIC ACID SUPPLEMENTATION RESCUES HIGH FAT DIET-INDUCED ALTERATIONS IN HIPPOCAMPAL INFLAMMATION AND IRSS EXPRESSION 


\subsection{Introduction}

Adult neurogenesis is a sensitive process that is very susceptible to different toxic insults, such as the presence of $A \beta$ plaques (characteristic in $A D$ ) ${ }^{[248]}$, insulin resistance ${ }^{[249]}$, or inflammation ${ }^{[250]}$. Impairment of neurogenesis is associated with alterations of hippocampaldependent functions, including spatial awareness, long-term memory, emotionality, and mood $[251,252]$

For more than 20 years, neuroinflammation has been considered to underlie the onset of neurodegenerative disorders ${ }^{[253]}$, including $\mathrm{AD}^{[254-257]}$. Importantly, neuroinflammatory processes resulting from obesity and stress can induce insulin resistance ${ }^{[258]}$. These processes cause a considerable increase of reactive microglia, which secrete a variety of cytokines (e.g. TNF $\alpha$ ), which activate specific intracellular cascades, including IKK $\beta$ and JNK ${ }^{[259]}$. Aside from this, a large body of evidence has accumulated correlating insulin resistance induced by chronic inflammatory processes and the development of the disease ${ }^{[260]}$. In contrast, numerous preclinical and clinical studies have shown how insulin sensitization improved cognitive damage, cytokine levels, and mitochondrial function after a fat enriched-diet period ${ }^{[261,262]}$.

Interestingly, insulin/IGF-1 signalling has been shown to protect against these toxic insults and improves neurogenesis ${ }^{[263]}$. In line with this, physical exercise improves insulin sensitivity and increases hippocampal neurogenesis ${ }^{[264,265]}$. The mechanisms of the exercise-induced improvement of neurogenesis may be a result of several factors, importantly the augmented blood flow to the brain ${ }^{[266]}$ with the consequent affluence of growth factors, in particular IGF-1 ${ }^{[267]}$. Highlighting the importance of IGF-1, pilocarpine-induced epilepsy has been shown to increase the IGF-1 expression via cAMP response element-binding (CREB) protein stimulation in the SGZsurrounding microglia, improving neurogenesis ${ }^{[268]}$. Another important factor in neurogenesis is the BDNF ${ }^{[269]}$. In fact, there is evidence that hippocampal BDNF expression is also augmented by exercise ${ }^{[270]}$. Furthermore, a crosstalk between BDNF and IGF-1 has been reported to play an important role in exercise-induced neurogenesis ${ }^{[271]}$.

Insulin and IGF-1 signalling cascades are very similar: both peptides exert their actions through tyrosine kinase receptors via the subsequent activation of the IRSs ${ }^{[272-274]}$. Of the four known receptor substrate isoforms, the IRS1 and IRS2 isoforms are highly expressed in the brain ${ }^{[121,275]}$. Insulin and IGF-1 receptors are expressed in dentate gyrus in rat and mouse brains ${ }^{[276-279]}$, but the specific expression of the IRS in these areas is largely unknown. Neuroinflammation may induce insulin resistance through IRS inactivation by specific serine phosphorylation. In humans, IRS1 P-Ser 307 may determine insulin resistance in type 2 diabetes ${ }^{[50,280]}$. One of the consequences of pSer in IRS1 is to increase the degradation of the protein using ubiquitination ${ }^{[47]}$. However, this process does not appear to occur for IRS2 degradation ${ }^{[281]}$, which suggests differential regulation of both proteins, at least in certain cell types.

In addition to exercise, other factors, such as phytohormones have been proposed as neuroprotectors, precisely due to their insulin sensitizer activity and anti-inflammatory properties ${ }^{[282]}$. For instance, ABA is a PPAR $\gamma$ agonist ${ }^{[147]}$ that can improve glucose tolerance in obesity models, reduce neuroinflammation, and restore HFD-induced neurological alterations ${ }^{[118,152,161]}$.

In this study, we evaluate the effects of HFD-induced neuroinflammation and ABA treatment in the expression levels of several members of the insulin/IGF-1 pathway, along with inflammation markers, in the HPC. One of the well-known effects of HFD exposure is the reduction of adult hippocampal neurogenesis ${ }^{[283]}$; therefore, we evaluated whether ABA treatment could rescue the HFD-induced reduction in hippocampal neurogenesis via its anti-inflammatory and insulin sensitizing properties. 


\subsection{Materials and Methods}

\section{Animals and Diet}

Eight-week-old male Wistar rats (Janvier Labs, Saint-Berthevin, France) were kept at the animal facility of the University Jaume I. The procedures followed directive 86/609/EEC of the European Community on the protection of animals used for experimental and other scientific purposes. The experiments were approved by the Ethics Committee of the University Jaume I (approval number 2014/VSC/PEA00209). The animals were maintained on a $12 \mathrm{h:12} \mathrm{h}$ light-dark cycle and housed in pairs to reduce stress due to social isolation. Rats were divided randomly into four experimental groups: SD, control animals fed the standard rodent diet (Ssniff, Soest, Germany); SD-ABA, animals fed standard diet supplemented with ABA (Fernandez-Rapado, Spain) in their drinking water (20 mg/L); HFD, animals fed an HFD (5736 kcal/kg, Ssniff), and HFD-ABA, animals fed an HFD and that had ABA in their drinking water $(20 \mathrm{mg} / \mathrm{L})$. We have used ABA concentration as described previously ${ }^{[118]}$. The four groups were fed ad libitum for 12 weeks.

\section{Immunoblotting}

Rats were lightly anesthetized (Dolethal, $200 \mathrm{mg} / \mathrm{Kg}$ Vetoquinol S.A.,Madrid, Spain) and then killed by decapitation. Brains were rapidly removed and frozen in cold isopentane (SigmaAldrich, St Louis, MO, USA). Hippocampi were dissected at $-15{ }^{\circ} \mathrm{C}$ (using a cryostat) to preserve the protein phosphorylation. Tissue was lysed in a RIPA buffer containing protease and phosphatase inhibitors (SERVA Electrophoresis, Heidelberg, Germany). Mechanical tissue lysis was achieved using a sonicator (Hielsher Ultrasound Technology, Teltow, Germany). Thirty micrograms of the total protein were subjected to SDS-PAGE, transferred to Immobilon-P membranes (MERCK Millipore, Darmstadt Germany), which were blocked for nonspecific binding and incubated with primary antibodies: anti-phospho IKK $\alpha \beta$ (Santa Cruz Biotechnology, Santa Cruz, CA, USA; 1:500); anti-phospho TAU (Abcam, Cambridge, UK; 1:1000); anti-IRS1 (EMD Millipore, Temecula, CA, USA; 1:500); anti-IRS2 (Cell Signaling, Danvers, MA, USA 1:1000), and anti- $\beta$-Actin (Sigma-Aldrich, St Louis,MO, USA; 1:2000) overnight at $4^{\circ} \mathrm{C}$. After several washes with a washing buffer containing $0.3 \%$ Triton $\mathrm{X}-100$, the membranes were incubated for 1 $\mathrm{h}$ at RT with peroxidase-conjugated secondary antibodies (anti-rabbit and anti-mouse, Jackson Immunoresearch, Suffolk, UK). Staining was developed using ECL (BioRad,Hercules CA, USA), and digital images were captured with a charge-coupled device imager (IMAGEQUANT LAS 4000, GE Healthcare Little Chalfont, UK). Immunoreactive bands were quantified with Image J blots toolkit software (National Institutes of Health, Baltimore, MD, USA), which was normalized to the $\beta$-Actin signal in each sample. Data were expressed as a percentage of the control (standard diet) normalized signal as the mean plus and minus the standard error of mean (SEM).

\section{Immunohistochemistry}

Rats were anesthetized with pentobarbital (120 mg/kg Eutanax, Fatro, Barcelona, Spain) and transcardially perfused with saline $(0.9 \%)$, followed by a $4 \%$ PFA fixative in $0.1 \mathrm{M}$ phosphate buffer, $\mathrm{pH}$ 7.4. After perfusion, the brains were removed and post-fixed overnight at $4{ }^{\circ} \mathrm{C}$ in PFA, followed by $48 \mathrm{~h}$ in a $30 \%$ sucrose solution for cryoprotection. Sliding Microtome Leica SM2010R (Leica Microsystems, Heidelberg, Germany) was used to obtain $40-\mu \mathrm{m}$ thick coronal frozen sections. The brains were cut in rostrocaudal direction; six series of slices were collected from each brain and stored at $-20{ }^{\circ} \mathrm{C}$. Sections were rinsed twice in $0.05 \mathrm{M} \mathrm{TBS}, \mathrm{pH} 8.0$, containing $0.2 \%$ Triton X-100 at RT. Slices were incubated in blocking solution (containing 2\% donkey serum and $4 \%$ serum albumin bovine for $1 \mathrm{~h}$ ); anti-Doublecortin (DCX) (Santa Cruz Biotechnology, Santa Cruz, CA, USA; 1:1000) was used. After several washes, the slides were incubated with the biotinylated secondary antibody (Jackson 1: 200) in TBS with $0.2 \%$ Triton X-100. Then sections were rinsed $3 \mathrm{x}$ for $10 \mathrm{~min}$ and transferred to a 1:50 avidin-biotin-horseradish peroxidase complex solution for 90 min (VECTASTAIN® Elite ${ }^{\circledR}$ ABC-HRP Kit; Peroxidase, Standard, USA). Then, sections were rinsed $2 \mathrm{x}$ for $10 \mathrm{~min}$ in TBS and 2x for $10 \mathrm{~min}$ in $0.05 \mathrm{M}$ 7.6 $\mathrm{Ph}$ Tris Buffer (TB). Color reaction was achieved by incubation with DAB (Sigma-Aldrich, St Louis, MO, USA) and 2 
$\mu \mathrm{l}$ of $\mathrm{H}_{2} \mathrm{O}_{2}$ in TB for 15-20 min. The reaction was stopped by several rinses in phosphate buffered saline solution. Finally, the slices were mounted on gelatinized slides and air dried overnight. Then, sections were rehydrated, alcohol-dehydrated, xylene-cleared and coverslipped with a DPX mounting medium.

\section{Image Analysis}

Images were taken by a Nikon optical microscope (Nikon, Tokyo, Japan) attached to a Leica camera (Leica Microsystems), which was connected to the Leica software (Leica Microsystems), to acquire the images. To quantify the DCX-positive neurons, six images were taken for each anatomical subdivision of the HPC in the four treatments (HFD, HFD-ABA, SD, and SD-ABA). The DCX-positive neurons were counted with Image $\mathbf{J}$ (National Institute of Health, Baltimore, MD, USA) at levels rostral (Bregma $-3.72 \mathrm{~mm}$ ) and caudal (Bregma $-6.00 \mathrm{~mm}$ ) levels (Paxinos and Watson ${ }^{[284]}$. Data were expressed as the mean and the SEM of the DCX-positive neurons $(n=6)$. The researcher performing the quantification of DCX-positive neurons was blind to the experimental condition.

\section{RNA Extraction and Real-Time Quantitative Polymerase Chain Reaction (RT-qPCR)}

Total RNA was extracted from the rat HPC $(n=5-11)$ and homogenized in $500 \mu \mathrm{L}$ of lysis buffer according to the Norgen Fatty Tissue RNA Purification Kit (Norgen Biotek Corp, product no. 36200, ON, Canada). Genomic DNA was removed using a spin-column process during the RNA extraction. In addition, DNAse I treatment (Thermo-Fisher Scientific, Waltham, Massachusetts, USA) was performed to ensure the complete removal of genomic DNA. RNA samples were eluted in $50 \mu \mathrm{L}$ of nuclease-free water and reverse transcribed to cDNA using a PrimeScript ${ }^{\mathrm{TM}}$ RT reagent kit (Takara, Shiga, Japan) following the manufacturer's instructions. Primers were designed using the Primer3 software tool (http://primer3.ut.ee/) (Table 4.1). RTqPCR reactions were carried out using SYBR PREMIX Ex Taq (Tli RNase H Plus) (TAKARA Bio Inc., Shiga, Japan) in an Applied Biosystems StepOne Plus ${ }^{\mathrm{TM}}$ Real-Time PCR System (Foster City, CA, USA). At the end of each PCR reaction, a melting curve stage was performed to confirm that only one PCR product was amplified in these reactions. The relative gene expression to SD was calculated by using the $2^{-\Delta \Delta \mathrm{Ct}}$ method for each reaction and by using the housekeeping gene GAPDH as internal control.

Table 4.1. Primers sequences obtained from Primer3 software tool.

\begin{tabular}{llll}
\hline Genes & Accession number & Forward primer (5' -> 3') & Reverse primer (5' $>$ > 3') \\
\hline GAPDH & NM_017008.4 & TGCCCCATGTTTGTGATG & TGGTGGTGCAGGATGCATT \\
INSR & M29014.1 & TCCTAAAGATCCGTCGCTCC & AAGAGCTTGCCCTGAGTGAT \\
IGF-1R & NM_052807.2 & TGTCCTCTCGGCATCAAACT & TATCACCACCGCACACTTCT \\
IRS-1 & NM_012969.1 & ATTGGAGGTGGGTCTTGCAG & TGGGGATCTTCTGGGCCATA \\
IRS-2 & NM_001168633.1 & TCACCACAGGACACAGATGC & GCATGAAGTGTGGCAAACGT \\
APP & NM_019288.2 & CCCCAAGATCCGGTTAAACT & TACTTGTCGACTGCGTCAGG \\
BDNF & NM_001270638.1 & GAGACAAGAACACAGGAGGAAA & CCCAAGAGGTAAAGTGTAGAAGG \\
TNFa & NM_012675.3 & GACCCTCACACTCAGATCATCTTCT & TGCTACGAGGTGGGCTACG \\
\hline
\end{tabular}

Statistics

Data were expressed as mean \pm SEM and subjected to a Kolmogorov-Smirnov normality test, and only when the test was positive for normality $(\alpha=0.05)$ were the data analyzed using a parametric one-way ANOVA followed by a post-hoc Newman-Keuls test. To evaluate the associations between variables, we used a two-tailed Pearson correlation test. The correlation coefficient $r$ values ( \pm 0.1 to \pm 0.3 ) reveal a weak association, are a ( \pm 0.3 to \pm 0.5$)$ medium, and 
are higher than \pm 0.5 strong correlation ${ }^{[285-287]}$. Positive or negative $r$ values mean positive or negative correlations, respectively ${ }^{[288]}$.

\subsection{Results}

\subsubsection{A HFD alters hippocampal IRS1 and IRS2 mRNA expression. ABA treatment can prevent these changes}

We found that TNFa levels were increased significantly by HFD treatment, and ABA administration, along with a HFD, prevented this significant increase $\left(F_{3,39}=9.363\right)$. Similarly, we observed that the BDNF mRNA level in the HPC was significantly reduced in HFD-treated animals compared to $\mathrm{SD}$ and that the treatment with $\mathrm{ABA}$ in the drinking water prevented this reduction $\left(F_{3,16}=5.898\right)$. Interestingly, the APP mRNA expression levels also showed a significant increase with HFD exposure $\left(F_{3,19}=4.524\right)$.

Next, we wanted to evaluate the effects of a HFD on the proteins' expression of the insulinsignalling pathway. Even though it is widely accepted that a HFD and neuroinflammation induces insulin resistance, whether a HFD affects mRNA levels of IR, IRS1, and IRS2 is largely unknown. In our model, we observed a significant reduction of IRS2 mRNA levels in the HPC of rats fed a HFD with respect to SD (Table 4.2). Most interestingly, ABA treatment prevented this significant reduction in the hippocampal IRS2 levels in rats fed a HFD $\left(F_{3,37}=5.911\right)$. Moreover, there was a significant increase in the mRNA levels of IRS1 in those animals fed a HFD, which can also be rescued by $\mathrm{ABA}$ administration $\left(F_{3,22}=4.301\right)$ (Table 4.2). Hence, these results suggest that the modulation of IRS1 and IRS2 gene expression may be different, depending on the environmental conditions, which suggests that these proteins may be involved in different cellular needs, in insulin signalling, and in neuroinflammation. IR did not change with diet or ABA treatment; however, the IGF-1R did show a change in those control samples that were treated with ABA $\left(F_{3,16}=3.025\right)$ (Table 4.2).

Table 4.2. Hippocampal mRNA expression of neuroinflammation markers and Insulin/IGF-1 pathway genes.

\begin{tabular}{lcccc}
\hline Genes & HFD & HFD-ABA & SD & SD-ABA \\
\hline TNF $\alpha(n=7-15)$ & $1.67 \pm 0.16$ & $1.19 \pm 0.14 *$ & $1.01 \pm 0.04 * * *$ & $0.99 \pm 0.04 * *$ \\
BDNF (n=5) & $0.59 \pm 0.04$ & $0.85 \pm 0.10$ & $1.00 \pm 0.02 * *$ & $0.87 \pm 0.09$ \\
APP (n=5-6) & $1.8 \pm 0.29$ & $1.41 \pm 0.22$ & $1.00 \pm 0.01 * *$ & $0.95 \pm 0.13 *$ \\
IRS1 (n=7) & $1.28 \pm 0.08$ & $1.06 \pm 0.04$ & $1.01 \pm 0.02 *$ & $1.06 \pm 0.08$ \\
IR (n=5-7) & $0.97 \pm 0.07$ & $0.95 \pm 0.14$ & $1.01 \pm 0.01$ & $0.99 \pm 0.04$ \\
IGF-1R (n=5) & $1.24 \pm 0.14$ & $1.11 \pm 0.10$ & $1.00 \pm 0.01$ & $0.80 \pm 0.13 *$ \\
IRS2 (n=7-14) & $0.59 \pm 0.06$ & $1.02 \pm 0.09 *$ & $1.00 \pm 0.06 * *$ & $0.83 \pm 0.15$ \\
\hline
\end{tabular}

\subsubsection{ABA can effectively restore HFD-induced reduction in hippocampal IRS2 protein}

To evaluate the effect of a HFD on protein expression in the HPC, we measured the levels of both IRS1 and IRS2 and the inflammatory markers phosphorylated inhibitor of nuclear factor kappa-B kinase subunit (pIKK) ${ }^{[289]}$ and TAU (pTAU) ${ }^{[290]}$ using a western blot (Fig. 4.1A). We observed that IRS2 protein levels were significantly lower in the HFD group $(0.61 \pm 0.05 ; n=11)$ compared to SD group $(0.99 \pm 0.02 ; \mathrm{n}=9)$, HFD supplemented with ABA group $(1.00 \pm 0.16 ; \mathrm{n}=$ $12)$, and the SD-ABA group $(1.29 \pm 0.17 ; \mathrm{n}=7)$, ANOVA $\left(F_{3,35}=4.897 ; p=0.006\right)$ (Fig. 4.1B). In contrast, hippocampal IRS1 protein levels from matched samples were not different from each other [HFD $(0.97 \pm 0.12 ; n=5)$, HFD-ABA $(1.05 \pm 0.12 ; n=5)$, SD $(0.96 \pm 0.02 ; n=5)$, SD-ABA $(1.31 \pm 0.25 ; n=5)$ ] (Fig. $4.1 \mathrm{C})$. On the other hand, pIKK did not show any significant differences 
between groups [HFD $(1.38 \pm 0.32 ; n=6)$, HFD-ABA $(1.28 \pm 0.20 ; n=6), \operatorname{SD}(0.98 \pm 0.01 ; n=$ 7), SD-ABA (1.15 $\pm 0.07 ; n=7)$ ] (Fig. 4.1D). We found that ABA increased pTAU expression in SD-ABA animals $(1.37 \pm 0.19 ; n=7)$ with respect to the SD group $(0.99 \pm 0.02 ; n=9)$, that the HFD-treated animals had lower levels, although not significantly lower $(0.68 \pm 0.12 ; n=11)$, and ABA added to HFD group $(0.88 \pm 0.07 ; \mathrm{n}=11)$ did not significantly alter the pTAU levels, ANOVA $\left(F_{3,34}=6.012 ; p=0.002\right)$ (Fig. 4.1E).

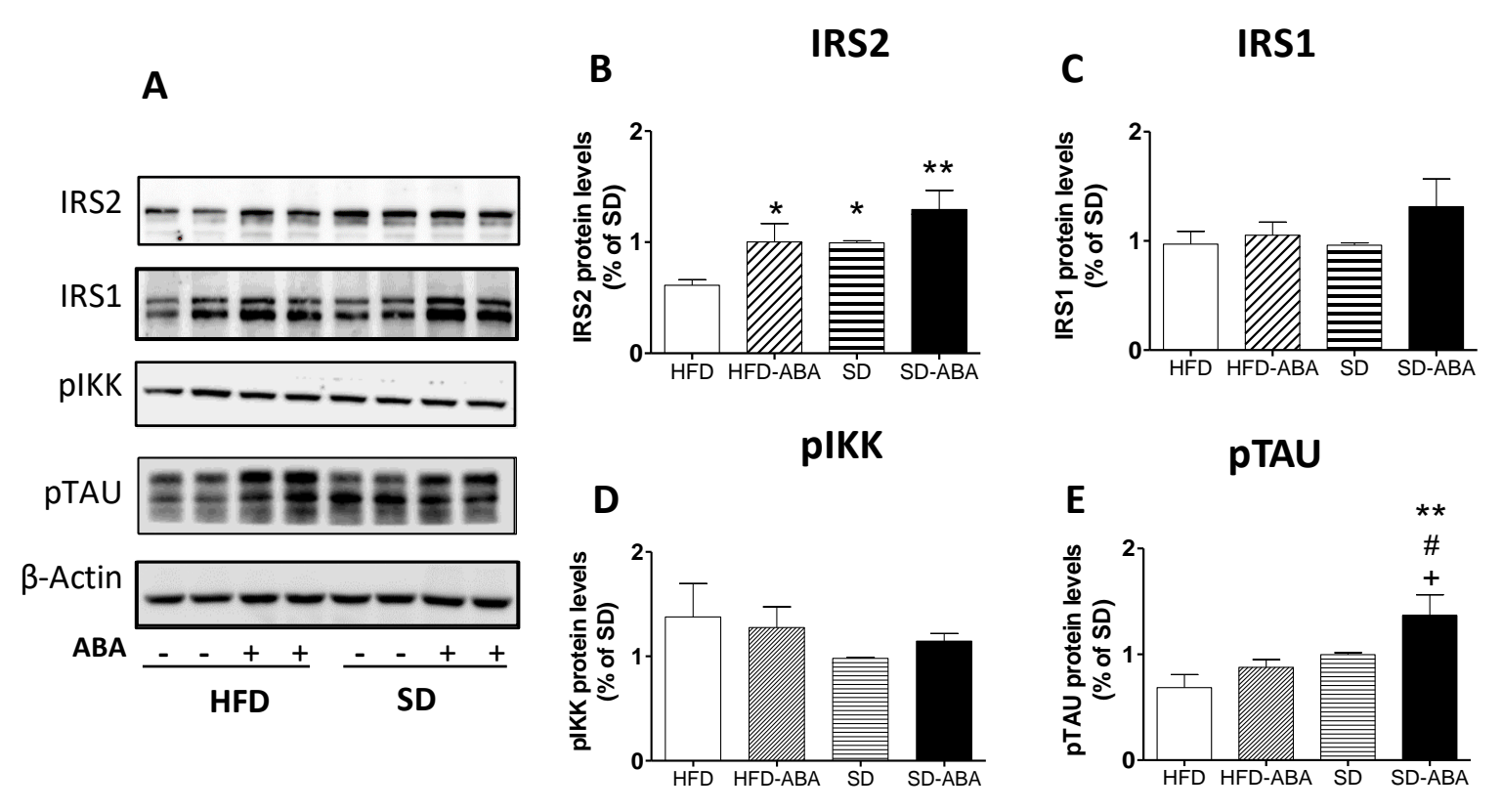

Fig. 4.1. A western blot analysis was carried out to evaluate the expression of the proteins in the hippocampus. Representative images of IRS2, IRS1, pIKK, pTAU, and loading control $\beta$-Actin (A). Quantifications of IRS2 protein levels (B), IRS1 (C), pIKK (D), and pTAU (E). HFD (white columns); HFD-ABA (diagonal lines); SD (horizontal lines), and SD-ABA (black columns). Data are represented as the mean \pm SEM $(n=6)$. The ANOVA was followed by a post-hoc NewmanKeuls test $(\mathrm{n}=5-11){ }^{*}, p<0.05 ;{ }^{* *}, p<0.01$ vs. HFD; \#, $p<0.05$ vs. HFD-ABA;,$+ p<0.05$ vs. SD.

\subsubsection{ABA rescues the HFD-induced reduction in hippocampal neurogenesis}

To evaluate hippocampal neurogenesis, we quantified DCX, which was expressed in differentiating and migrating neurons during embryonic and postnatal development ${ }^{[291]}$ using immunohistochemistry in the rostral (Bregma $-3.72 \mathrm{~mm}$ ) and caudal HPC (Bregma $-6.00 \mathrm{~mm}$ ) (Fig. 4.2A), following the rat brain atlas ${ }^{[284]}$. Data were expressed as the mean of DCX-positive neurons \pm SEM. In the rostral HPC, we found that the number of DCX-positive neurons decreased significantly in HFD-fed rats $(131.9 \pm 41.4 ; n=6)$ compared to SD-fed controls $(275 \pm 34.15 ; n=$ $6)$. The ABA treatment rescued the DCX expression in HFD-ABA animals $(339.1 \pm 43.99 ; \mathrm{n}=6)$ and did not alter the DCX in SD animals $(257.2 \pm 34.15 ; \mathrm{n}=6)$. ANOVA $\left(F_{3,20}=6.150 ; p=\right.$ 0.0039)] (Fig. 4.2B). There were representative images of DCX-positive neurons in HFD (Fig. 4.2C), HFD-ABA (Fig. 4.2D), SD (Fig. 4.2E), and SD-ABA (Fig. 4.2F). An identical pattern was followed in the caudal HPC (data not shown). 
A

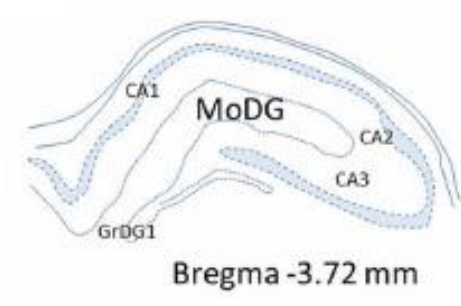

B

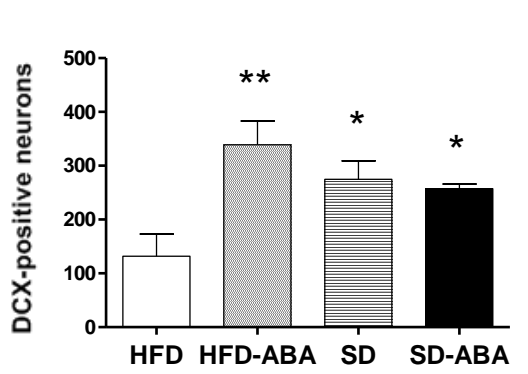

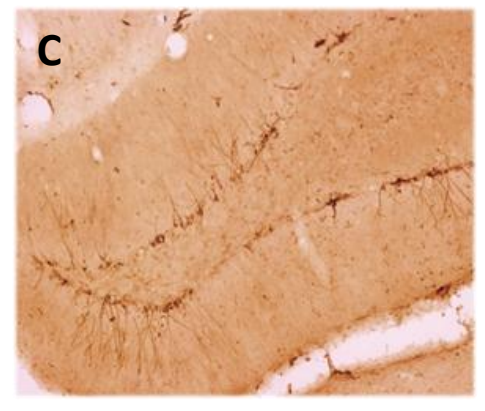
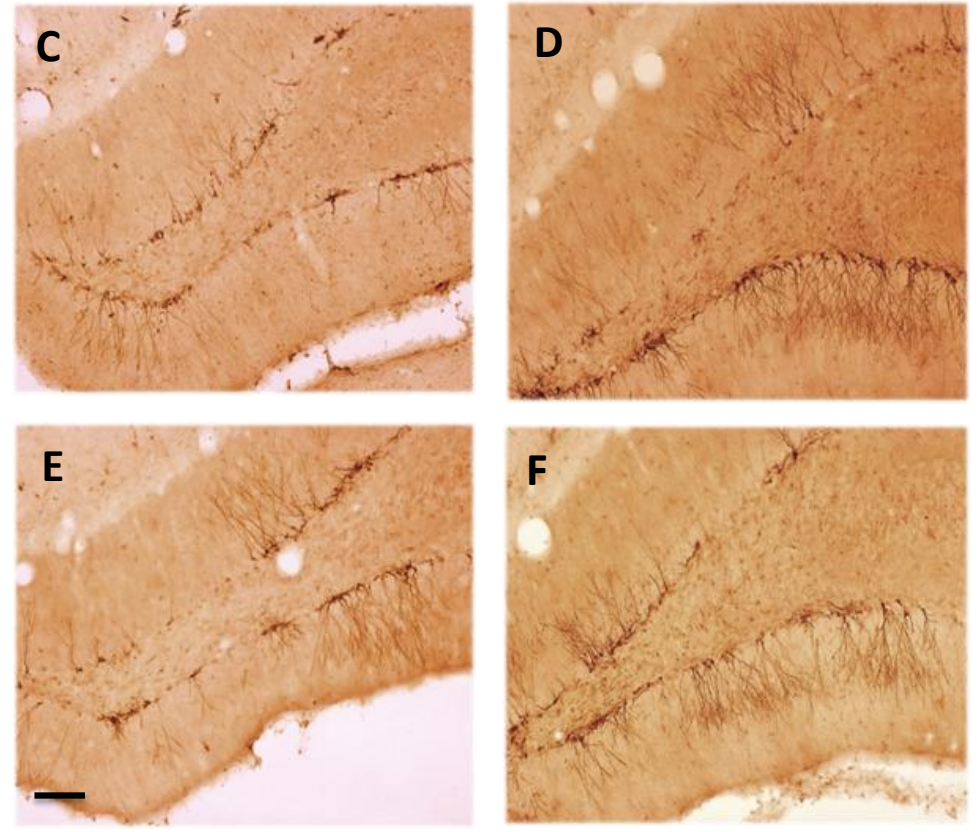

Fig. 4.2. ABA rescues the HFD-induced reduction in the number of DCX-positive neurons in the hippocampus. A Patxinos drawing of a rostral hippocampus (Bregma $-3.72 \mathrm{~mm}$ ) (A). The quantification of DCX-positive neurons (B). Data are represented as the mean \pm SEM $(n=6)$ and analyzed using a one-way ANOVA followed by post-hoc Newman-Keuls test. *, $p<0.05$; **, $p<0.01$ with respect to HFD. The representative images are of DCX imunolabeling in HFD (C), HFD-ABA (D), SD (E), and SD-ABA (F). Calibration bar, $100 \mu \mathrm{m}$.

\subsubsection{IRSI and IR21 mRNA levels correlate differently with TNF $\alpha, B D N F$ and APP}

We have observed that diet and ABA treatment can differently affect the expression of IRS1 and IRS2. To further understand the relationships of IRS expression and other biomarkers of inflammation, we aimed to ascertain if there was a relationship between the levels of IRS1 and IRS2 gene expression with TNF $\alpha$, APP, and BDNF. We observed that IRS1 correlates positively with TNF $\alpha$ (Pearson $r=0.574 ; p=0.02$ ) (Fig. 4.3A), whereas IRS2 correlates negatively with TNF $\alpha$ (Pearson $r=-0.498 ; p=0.05$ ) (Fig. 4.3D). Interestingly, IRS1 showed a positive correlation with APP (Pearson $r=0.672 ; p=0.003$ ) (Fig. 4.3B). In contrast, IRS2 did not correlate with APP either way (Fig. 4.3E). None of the substrates showed any correlation with BDNF levels (Fig. 4.3C and $\mathrm{F}$, respectively). 

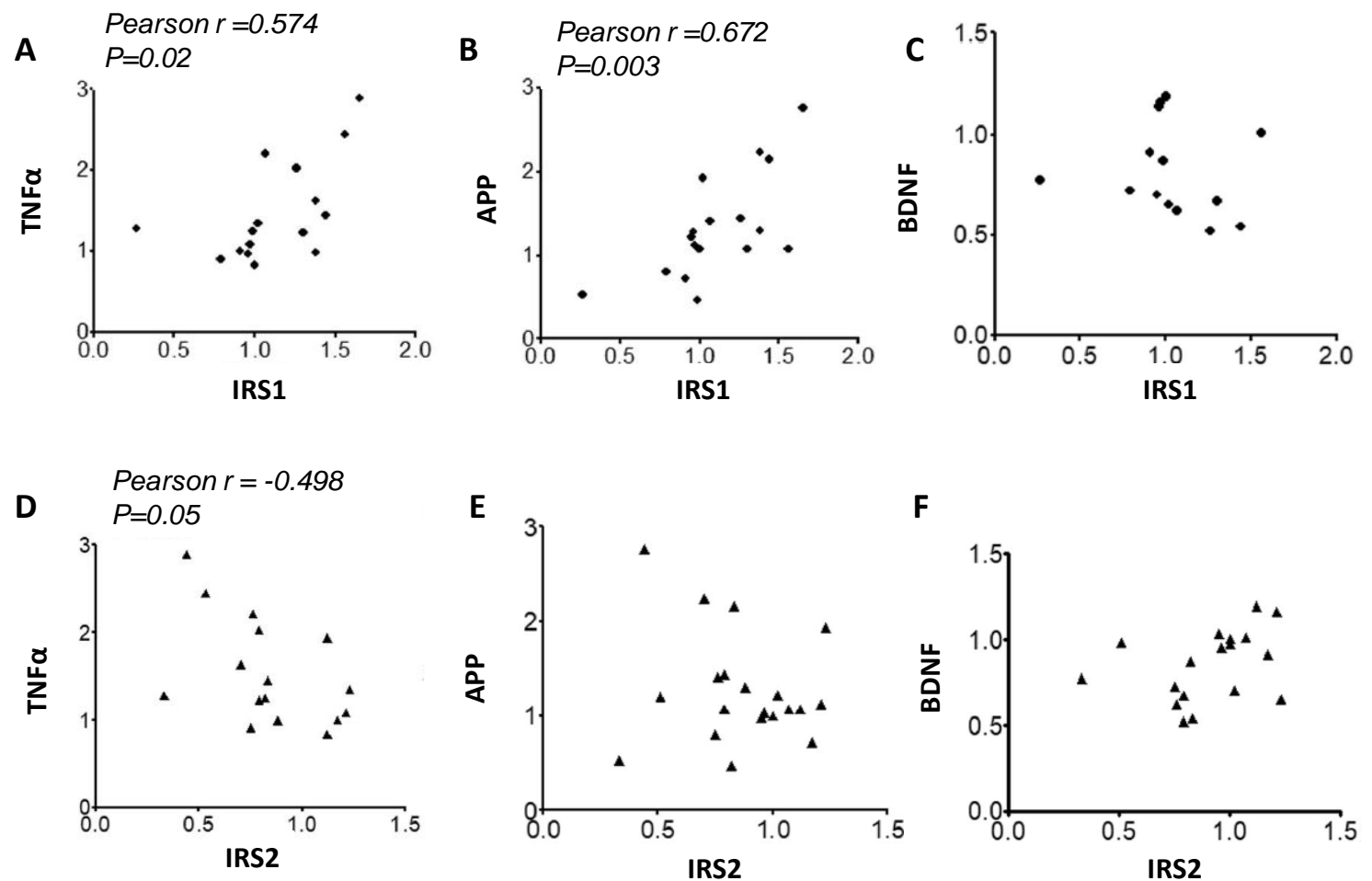

Fig. 4.3. The correlation of IRS1 and IRS2 with TNF $\alpha$, APP, and BDNF. The IRS1 expression correlates positively with the TNFa (A) and APP (B) expression. IRS1 does not correlate with the BDNF expression (C). The IRS2 gene expression correlates negatively with $\mathrm{TNF} \alpha$ (D) and does not correlate with the APP (E) or BDNF (F) expression.

\subsubsection{The IRS1 expression correlates with weight gain but IRS2 does not}

Due to the different patterns of expression showed by both isoforms of IRS, we aimed to elucidate if there was a relationship between the percentage of weight gain and the IRS expression. Interestingly, IRS1 showed a positive correlation with the percentage of weight gain (Pearson $r=$ $0.498 ; p=0.019$ ) (Fig. 4.4A). However, the IRS2 expression did not show any significant correlation when plotted versus the percentage of weight gain (Fig. 4.4B).

A

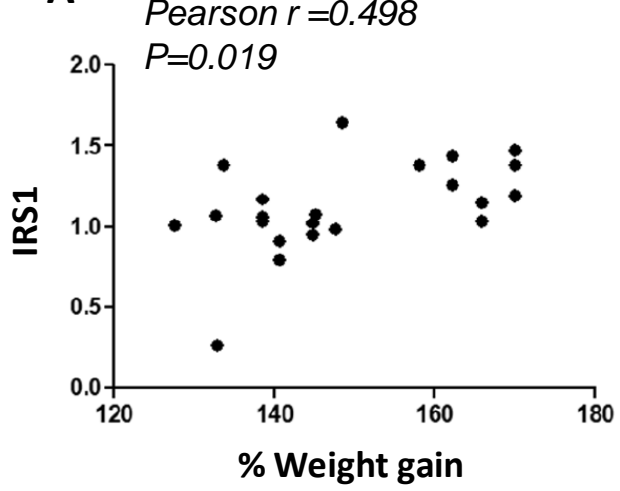

B

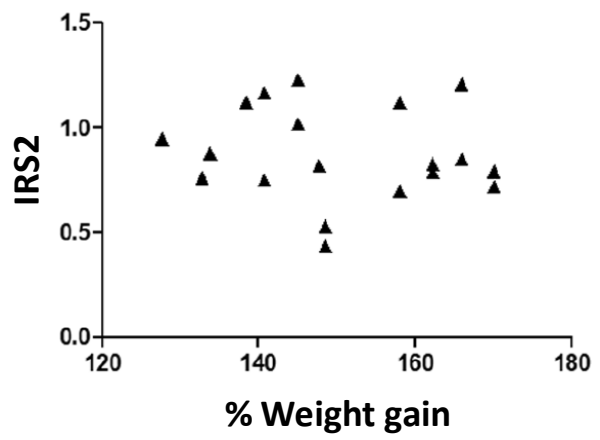

Fig. 4.4. The correlation of IRS1 and IRS2 versus the percentage of weight gain. The IRS1 expression correlates positively with the percentage of weight gain (A). There is no significant correlation between IRS2 and the percentage of weight gain (B). 


\subsection{Discussion}

Excessive caloric intake can induce neuroinflammation and insulin resistance. Both processes are considered major risk factors that lead to cognitive alterations ${ }^{[219,220,292]}$.

In this study, we have made use of a well-established model of neuroinflammation -feeding rats a HFD for 3 months- to study its effects on the insulin-signalling pathway. In addition, we have evaluated the effects of the phytohormone ABA in hippocampal insulin signalling. ABA targets PPAR $\gamma$ in a manner similar to the TZDs class of anti-diabetic drugs ${ }^{[222,293]}$ and curcumin, a wellknown potent anti-inflammatory molecule ${ }^{[294]}$ and also PPAR $\gamma$ agonist ${ }^{[295]}$. In fact, given their capability to reduce inflammation, this family of PPAR $\gamma$ agonist molecules has been proposed as a new class of molecules with which to treat central nervous system disorders ${ }^{[296]}$.

We have analyzed the mRNA of IR and IGF-1R and their substrates, IRS1 and IRS2. We found no changes in hippocampal IR or IGF-1R in the HPC of HFD-fed animals compared to the controls. However, our study shows for the first time that a HFD can reduce IRS2 expression in hippocampal tissue, both at the messenger RNA and protein levels, therefore providing an additional plausible mechanism of hippocampal insulin resistance in obesity and metabolic syndrome. These results are also in line with previous reports showing that IRS2 is reduced in dorsal root ganglia from diabetic (insulin resistance) rodent models ${ }^{[297]}$. Moreover, in the gastrocnemius muscle, IRS2 expression was found to be significantly reduced in diabetic animals compared to the controls ${ }^{[298]}$. On the contrary, we observed that HFD increased IRS1 levels. These results may be paradoxical; however, they are in line with earlier reports indicating that IRS1 mRNA is increased in the HPC of APP/PS1 (presenilin 1) transgenic mice compared to agedmatched controls ${ }^{[299]}$.

Interestingly, we have observed that treatment with ABA restored IRS1 and IRS2 expression, which could account for beneficial effects improving the cognitive performance of HFD-fed animals ${ }^{[118]}$. These results are supported by studies showing that PPAR $\gamma$ agonists can restore pathological IRS levels, that curcumin reduced the IRS1 levels in double APP/PS1 transgenic mice ${ }^{[299]}$, and that pioglitazone increased the pancreatic IRS2 expression in a model of diabetic transgenic mice ${ }^{[144]}$.

To our knowledge, our study reports for the first time that HFD alters hippocampal IRS1 and IRS2 expression and that supplementation with ABA is capable of restoring these alterations.

Protein expression was studied using immunodetection and confirmed the alterations observed in the IRS2 mRNA levels, but not IRS1 mRNA. This discrepancy in messenger and protein expression could be since the IRS1 protein levels may be regulated by degradation, and, therefore, the increase in mRNA is not being detected in the protein. Paradoxically, pIKK and pTAU were not found to be increased in a HFD as expected given the fact that inflammation augments the phosphorylation of IKK ${ }^{[300,301]}$ and that insulin resistance results in tau hyperphosphorylation ${ }^{[302]}$. Nevertheless, supporting our results, pIKK levels are not altered in HFD-treated rats ${ }^{[303]}$, indicating that pIKK may not be a universal and reliable marker for neuroinflammation. However, further studies should be carried out to elucidate the role of pIKK in hippocampal HFD-induced inflammation. Similarly, future research will help to understand the conditions required for accumulation of pTAU derived from an insulin-resistance situation. It is plausible that time is an important factor for observing such accumulation.

Furthermore, we have observed that ABA treatment is capable of restoring a HFD-induced reduction in neurogenesis, as evaluated by DCX-positive neurons in the subgranular layer of the dentate gyrus. It is well accepted that a HFD reduces neurogenesis and that exercise and learning can improve it ${ }^{[304]}$. Consistent with our data, many insulin sensitizer molecules used against type 2 diabetes have been proven effective in restoring altered neurogenesis ${ }^{[305]}$. In addition, BDNF signalling is closely related to neurogenesis modulation, synaptic plasticity, and the formation and recall of hippocampal-dependent memories ${ }^{[306]}$. BDNF reduction has also been proposed as one of the earliest events in HFD-induced cognitive impairment ${ }^{[307,308]}$. In this study, in addition to 
showing impaired neurogenesis, we observed that the BDNF mRNA levels were reduced in the HPC with HFD and that ABA treatment could efficiently prevent this reduction. This is in line with previous studies reporting that neurogenesis and BDNF levels can be rescued using insulin and BDNF-sensitizing nutritional compounds ${ }^{[309]}$ and anti-inflammatory supplementation ${ }^{[310]}$. BDNF may be able to induce IRS1 and IRS2 tyrosine phosphorylation ${ }^{[311]}$ but, to date, it has not been reported to regulate IRS1 or IRS2 expression. In fact, we found no correlation between IRS1 and IRS2 with BDNF levels.

On the other hand, TNF $\alpha$, a well-accepted marker of neuroinflammation, insulin resistance, and cognitive impairment ${ }^{[312]}$, increases in the brain of HFD-treated animals ${ }^{[313]}$. As expected, we found that TNFa levels were increased in the HPC of HFD-treated rats and that ABA treatment significantly could prevent it. This result confirms that $\mathrm{ABA}$ is an effective anti-neuroinflammatory molecule. Furthermore, since TNF $\alpha$ levels are also closely linked to impaired neurogenesis and reduction in $\mathrm{BDNF}{ }^{[114]}$, our study confirms the close relationship of these processes with IRS expression.

APP transgenic mice have been extensively used as a model for AD in an attempt to overexpress a mutated protein that promotes oligomer aggregation ${ }^{[315]}$. We have found that endogenous APP levels are increased in the HPC of HFD-fed rats. It is plausible that this increment can favour $\mathrm{A} \beta$ accumulation ${ }^{[316]}$ and mediate some of the deleterious effects of a HFD in cognition. We found that ABA treatment could effectively counteract these effects, preventing the overexpression of APP. Other studies have also shown that supplementary nutrients can effectively downregulate APP endogenous expression ${ }^{[317]}$. Therefore, the anti-inflammatory and insulin-sensitizing actions also regulate APP expression, preventing further complications of $A \beta$ deposition.

Finally, to understand the relationship between IRSs, the biomarkers used in this study and obesity, we analyzed the normalized levels of IRS1 and IRS2 with TNF $\alpha$, APP, BDNF rat hippocampal tissue, and the percentage of weight gain. Interestingly, we observed the levels of TNF $\alpha$ correlate inversely with the expression of IRS2 and positively with IRS1. This fact strongly suggests that IRS1 and IRS 2 can be differentially affected by neuroinflammatory processes and therefore modulates distinct aspects of insulin resistance. Further confirming this hypothesis, we found that APP correlates positively with IRS1 but does not correlate at all with IRS2; thus, it is plausible that the signalling pathway that affects APP can target only IRS1 but not IRS2. Moreover, the percentage of weight gain correlates positively with IRS1 but not with IRS2 expression.

Considering all these data, we conclude that both IRS1 and IRS2 expression regulation, although modulating insulin and IGF-1 signalling, are totally independent of each other, suggesting that their implication in neuroinflammation and brain insulin resistance may also be different. Further studies should be carried out to establish the mechanism underlying these differences, such as location, protein interactions, and promoter sensitivity. These findings will be crucial for opening different methods of brain insulin resistance treatment.

Moreover, we confirm that ABA can effectively restore neuroinflammation and neurogenesis, supporting the beneficial effects on memory that we have reported previously ${ }^{[18]}$. In addition, we show that ABA can also influence IRS1 and IRS2 expression levels, providing a mechanistic link to how ABA can restore insulin signalling. 


\section{5}

Chapter 3

AAV DELIVERY OF SHRNA AGAINST IRS1 IN GABAERGIC NEURONS IN RAT HIPPOCAMPUS IMPAIRS SPATIAL MEMORY IN FEMALES AND MALE RATS 


\subsection{Introduction}

Insulin and IGF-1 actions are mediated by the insulin and IGF-1 receptors that can activate similar signalling pathways with pleiotropic actions in mammals. In the brain, both peptides have been shown to modulate cognitive function ${ }^{[318,319]}$. The signalling cascades elicited by insulin and IGF-1 are almost identical and both mediated by the scaffolding proteins, the IRS ${ }^{[71,272]}$. There are four IRSs known isoforms, being the IRS1 and IRS2 the most abundant and studied, up to date ${ }^{\text {[272] }}$. In spite the fact that both, IRS1 and 2 have been shown to mediate insulin and IGF-1 effects, some reports reveal that these isoforms may modulate different signalling cascades in the liver and skeletal muscle tissues ${ }^{[70,320]}$. Also, studies using knockout mice suggest that IRS1 and IRS2 modulate different physiological processes ${ }^{[320-324]}$. However, these differences have not been fully established within the central nervous system, and some paradoxical results indicate that whereas brain insulin resistance is mediated by dysfunctional IRS1 ${ }^{[75]}$ in other systems the reduction of the IGF-1/IRS2 pathway is suggestive of a neuroprotective role ${ }^{[325,326]}$. In optimal conditions, both IRSs are considered equally activated by the insulin and the IGF-1 receptors tyrosine kinase activity. Once these scaffolding proteins are phosphorylated in Tyr residues, they activate downstream signalling cascades, e.g. the PI3K/Akt pathway, Ras/ERK; and soluble tyrosine kinases from the Fyn family ${ }^{[327-333]}$. Several physio-pathological conditions induce resistance to insulin and IGF-1. Among others, neuroinflammation feed-forward inhibition induces IRS1 phosphorylation in serine residues by cytokines like IKK $\beta$ and JNK. In hyperinsulinemia, feedback inhibition via mTOR also phosphorylates IRS1 in Ser residues ${ }^{[47,48,334]}$. Interestingly, both chronic insulin and IGF-1 resistance in the brain are associated with the occurrence of $\mathrm{AD}{ }^{[335,336]}$. Furthermore, reduced responses to IR-IRS1 and IGF-1R-IRS2 linked to PI3K ${ }^{[76]}$ have been found in postmortem analysis in $\mathrm{AD}$ patients, compared to controls.

The specific and differential role of IRS1 or IRS2 isoforms in distinct brain networks, isolated from other comorbidities, such as chronic inflammation or AD linked genetic mutations, has not been fully established. Whether insulin resistance is a primary cause of cognitive dysfunction or it is a consequence of inflammatory conditions remains elusive. Thus, we aimed to understand the specific role of IRS1 in the adult healthy brain, in the absence of any other peripheral or central comorbidities. To that end, we made use of adeno-associated virus (AAV) expressing short hairpin ribonucleic acid (shRNA) targeting IRS1 (shIRS1) into the dorsal HPC of female and male rats. A rat model has been extensively used to study brain function with wellaccepted methods of behavioural evaluation. We evaluated the IRS1 silencing effect in specific hippocampal circuitries involved in spatial memory and the synaptic plasticity of infected neurons. We report for the first time that, downregulation of IRS1 in dentate gyrus hilar PV and SOM neurons impairs spatial memory but not induces anxiety or depressive symptoms. In addition, our results suggest that female rats may be more susceptible to brain insulin resistance than males, which may contribute to explain sex differences in the prevalence of neurodegenerative diseases. 


\subsection{Materials and Methods}

\section{Design and generation of shRNA constructs}

Several sequences specifically targeting IRS 1 were designed using the provided software on the Invitrogen webpage (https://rnaidesigner.thermofisher.com/ rnaiexpress/); parameters were modified to fit destination plasmid for shRNA cloning (pSM155). Best shIRS1 candidates were synthesized (ThermoFisher Scientific ${ }^{\circledR}$ ). Two shRNA constructs were cloned following the protocol described ${ }^{[337]}$. The sequences of the oligonucleotides used in this study are shown in Supplementary Figure S5.1 (annexes section).

\section{Construction and purification of recombinant AAV-CMV-shIRSIA-EGFP}

To generate the expression cassette, a PCR (Supreme NZY-Proof DNA polymerase, NZYtech, Lisbon, Portugal) targeting the shIRS1 and the enhanced green fluorescent protein (EGFP) was performed. Primers were designed with the ClaI and HindIII restriction sites. The PCR product was cloned into pJET 1.2 according to the manufacturer's instructions (CloneJET PCR Cloning Kit; ThermoFisher Scientific $\left.{ }^{\circledR}, M A, U S A\right)$. Insert was cloned by ClaI/HindIII enzyme restriction from the pJET 1.2 constructs and subcloned into the destination vector pAAV-CMV-GFP (Cell Biolabs INC, CA, USA) by ligation (T4 DNA Ligase; Takara Bio INC, Kyoto, Japan) overnight. Ligation was transformed into Endura ${ }^{\mathrm{TM}}$ competent cells (Lucigen, WI, USA), and positive colonies evaluated by $M s c I$ and/or EcoRV enzyme digestion. Finally, sequencing confirmed the absence of point mutations (Servicio Central Soporte Investigacion Experimental from the University of Valencia). pAAV-CMV-shIRSIA-EGFP was co-transfected with pHelper and pAAV2-Dj/8 (Cell Biolabs, INC.) into packaging Human embryonic kidney cells 293 (HEK293) cells to produce recombinant AAV2/DJ8-CMV-shIRS1A-EGFP. The resulting viral particle was purified by the iodixanol method as described in Addgene protocols for AAV production and purification ${ }^{[338]}$. Cell lines were purchased from Cell Biolabs, INC, and were free from mycoplasma.

\section{Animals and surgical procedures}

Female and male Wistar rats (Janvier Labs, Saint-Berthevin, France) between 250 and $550 \mathrm{~g}$ were kept at the animal facility of the University Jaume I. The procedures followed the directive 86/609/EEC of the European Community on the protection of animals used for scientific purposes. The experiments were approved by the Ethics Committee of the University Jaume I (scientific procedure 2019/VSC/PEA/0143). The animals were maintained on a $12 \mathrm{~h}$ light cycle and provided with food and water ad libitum. Animals were caged in pairs to reduce single caging -induced stress. Surgeries were done over one week, approx. 4 surgeries a day. Female and male cohorts were done in different phases to avoid confounding results.

For surgical procedures rats were first deeply anesthetized with ketamine (Ketamidor 50 $\mathrm{mg} / \mathrm{kg}$ i.p.; Merial Laboratories SA, Barcelona, Spain) and xylazine (Xilagesic $10 \mathrm{mg} / \mathrm{kg}$ i.p.; Lab Calier, Barcelona, Spain). Stereotaxic coordinates used to infuse the viral particle into the dorsal HPC bilaterally were AP $-4.36 \mathrm{~mm}, \mathrm{ML} \pm 2.5 \mathrm{~mm}, \mathrm{DV}-2.5 \mathrm{~mm}$ from bregma ${ }^{[284]}$. Bilateral injections were performed; $2 \mu \mathrm{L}$ of either AAV/DJ8-CMV-EGFP $(4.94 \mathrm{E}+11 \mathrm{GC} / \mathrm{mL})$ or AAV/DJ8CMV-shIRS1-EGFP $(1.02 \mathrm{E}+11 \mathrm{GC} / \mathrm{mL})$ per hemisphere. Animals were randomly divided into AAV control AAV shIRS1 groups and code labelled by an independent researcher. We used 7-8 rats per group and sex. This group size has been chosen based on our previous experience and the literature. Animal welfare was monitored although the procedure according to Ethical committee rules. Table and graph showing the subject's weight before the surgery and one month later, before behaviour tests are shown in Supplementary Table S5.1 (annexes section).

\section{Behavioural procedures}

In all the procedures rats were habituated to the testing room two days before performing the behaviour paradigm. Animals were recorded using a video tracking system (Smart 2.5.19, Panlab, 
Barcelona, Spain). Behavioural tests were conducted during the day, with a dim light. The researcher was blind to the group's condition. All animals performed these tests.

Open Field; Rats were placed in the open field facing one of the walls and allowed to freely explore the arena for $10 \mathrm{~min}$. The latency (time) to cross for the first time the centre quadrants with all four legs was quantified.

Novel Object Recognition (NOR); NOR experiment was conducted as described previously ${ }^{[18]}$. Briefly, rats were habituated to the experimental box before the test day. In the test day, the subjects were left to explore two identical objects, for 3 minutes (familiarization phase). After $1 \mathrm{~h}$ interval, the rats were back to the arena, and allowed to explore one of the previous objects (familiar) and a novel object for 3 min (test phase). "Exploration" is defined as time spent sniffing the object closer than $4 \mathrm{~cm}$, or touching it with the head oriented towards the object. Climbing over the object or running around it was not considered exploration. Data were expressed as the Discrimination Index (DI), defined as the time spent in the novel object, minus time spent in familiar one, divided by total time exploring. Thus, DI $=0$ means the animal spends equal time exploring both objects. $\mathrm{DI}=1$ or $\mathrm{DI}=-1$ would mean that the subject spends total time exploring the novel or the familiar, respectively.

T-maze; The T-maze test was performed as described previously ${ }^{[118]}$. Briefly, the animal is placed in the starting position and left to explore for $5 \mathrm{~min}$, with access to two of the three arms (familiarization phase). The rats were then returned to the home cage for a 2-h inter-trial interval and then placed back in the starting position, but now with access to all three arms for 5 min (test phase). The arm that was closed during the familiarization phase is considered the "novel" arm, the arm visited during the familiarization phase, is considered the "familiar" arm. Data expressed as the DI, as above.

Forced Swim Test; The forced swim test (FST) apparatus consisted of a Plexiglass cylinder with translucent walls $(30 \mathrm{~cm}$ in diameter and $50 \mathrm{~cm}$ high). The cylinder was filled with tap water at $23 \pm 1{ }^{\circ} \mathrm{C}$ and the water depth was adjusted so the rat cannot touch the bottom of the container. In the first session (pre-test phase), the rats were placed in the water-filled cylinder for $15 \mathrm{~min}$. Twenty-four hours later (test phase), each rat was returned to the FST apparatus for 5 min ${ }^{\text {[339]. }}$. Once each session was finished, the animals were placed in a drying cage with a heating pad under it and constant temperature that did not exceed $37^{\circ} \mathrm{C}$. Both days, the cylinder was placed at the same position in the room and the water was changed after every test to avoid any influence on the next rat. Three parameters were measured: "Immobile" was considered when the rat was floating without any movement, "Climbing" when the limbs were moved quickly breaking the surface of the water, "Swimming" when the rat moved the limbs in a paddling way.

\section{Immunofluorescence}

Immunofluorescence was performed as described previously ${ }^{[114]}$. Briefly, randomly chosen rats were anesthetized and transcardially perfused with saline $(0.9 \% \mathrm{NaCl})$ followed by fixative (4\% paraformaldehyde in $0.1 \mathrm{M} \mathrm{PB}, \mathrm{pH}$ 7.4). The other half of the brains was frozen for biochemical analysis. However, given the specificity of the AAV infection, considering that the surrounding neurons do express mRNA IRS1 we did not perform biochemical analysis.

After perfusion, the brains were removed, postfixed overnight and cryoprotected in $30 \%$ sucrose in $0.01 \mathrm{M}$ PBS pH 7.4 for 3 days. The brains were cut in rostrocaudal direction $(40 \mu \mathrm{m})$ using a sliding microtome Leica SM2010R (Leica Microsystems, Heidelberg, Germany). The following primary antibodies were used: goat anti-ChAT (MERCK Millipore, Darmstadt Germany; 1:700); mouse anti-calbindin (CB) (Swant, Marly, Switzerland; 1:3000); rabbit anti-calretinin (CR) (Swant; 1:2000); rabbit anti-parvalbumin (PV) (Swant; 1:5000); mouse anti-somatostatin (SOM) (Santa Cruz Biotechnology, Santa Cruz, CA, USA; 1:50); mouse anti-synaptophysin (SigmaAldrich, St Louis, MO, USA; 1:100); mouse anti-Glial Fibrillary Acidic Protein (GFAP) (SigmaAldrich, St Louis, MO, USA; 1:100); rabbit anti-IRS1 (Novus Biologicals, Abingdon, UK; 1:100). After overnight incubation, AAV-infected sections were rinsed and incubated for $2 \mathrm{~h}$ at RT with 
donkey anti-mouse $\mathrm{Cy} 3$, donkey anti-goat $\mathrm{Cy} 3$, and goat anti-rabbit $\mathrm{Cy} 3$ secondary antibodies (Jackson Immunoresearch, Suffolk, UK). Finally, sections were mounted on slides and coverslipped using Fluoromount-G mounting medium (Invitrogen, California, USA). For descriptive immunofluorescence, three animals per condition were done, and one example is shown in results. For IRS1+EGFP dual-labeling quantifications female rats 3 controls and 4 shIRS1 and male rats 7 controls and 7 shIRS1 were used and 2 dentate gyri per animal. For CR+AAV, $\mathrm{PV}+\mathrm{AAV}$, and SOM+AAV dual-labelling quantifications female rats 4 shIRS1 and male rats 7 shIRS1 were used and 1 dentate gyrus per animal. For synaptophysin quantifications female rats 3 controls and 4 shIRS1 were used and 10 different axonal fibers per animal.

\section{Imaging and analysis}

Fluorescence images were taken with a confocal scan unit with a module TCS SP8 equipped with argon and helio-neon laser beams attached to a Leica DMi8 inverted microscope (Leica Microsystems). Excitation and emission wavelengths for Cy3 were 433 and 560-618 nm respectively; Alexa488-labeled excitation wavelength was $488 \mathrm{~nm}$ and its emission at 510-570 nm. Serial $0.2 \mu \mathrm{m}$ scans were obtained in the Z-plane.

For the quantification of dual-labelling, we used a 20x lens. Image J software combined with loci. tools plugin (BIO-FORMATS, University of Wisconsin-Madison) was used to count the number of positively labelled cells in 20-32 Z-plane sections from control and shIRS1 groups of both females and males. For IRS1+EGFP dual-labelling quantifications, data were expressed as the percentage of double-labelled neurons (IRS1+AAV), divided by the total number of AAV-positive neurons. 3 AAV controls and 4 AAV shIRS1 females and 7 AAV controls and 7 shIRS1 males were analyzed by a researcher blind to the condition ( 2 dentate gyri per animal). For CR+EGFP, $\mathrm{PV}+\mathrm{EGFP}$, and SOM+EGFP dual-labelling quantifications, data were expressed as the percentage of double-labelled neurons (neuronal marker + EGFP), divided by the total number of neuronal marker-positive cells. 4 AAV shIRS1 females and 7 AAV shIRS1 males were analyzed by a researcher blind to the condition (1 dentate gyrus per animal).

For quantification of synaptophysin signal in AAV infected neurons, we used a 63x lens. Image $\mathbf{J}$ software combined with loci. tool plugin was used to count the number of synaptophysin points (minimum 3 pixels were considered) in one AAV-positive fiber in 10-15 Z-plane sections from control and shIRS1 groups. Three AAV controls and four AAV shIRS1 female animals, 10 different axons per animal were analyzed by a researcher blind to the condition. Data are calculated as the number of synaptophysin positive signals on the AAV-infected fiber, normalized to the area of the fiber.

The experimental outcomes from these procedures were, IRS1 mRNA expression, done in transfected cells. Behavioural changes in rats inoculated with the virus. And postmortem analysis of AAV tracing, colocalization quantification studies, and synaptophysin quantification.

\section{Statistical analysis}

The analysis was carried out with Graph Pad software (GraphPad Prism V8 software, GraphPad, La Jolla, CA, USA). Data reported as the mean \pm SEM and the " $n$ " the number of independent subjects. Data were subjected to Kolmogorov-Smirnov test for Gaussian distribution. If normality was confirmed, one-tailed or two-tailed Student's t-test with probability set at $\alpha<0.05$ was used, otherwise the non-parametric Mann-Whitney $T$ test was applied as indicated. Pearson test was used for correlation analysis. The correlation coefficient $r$ values $( \pm 0.1$ to \pm 0.3 ) reveal weak, values around $( \pm 0.3$ to \pm 0.5$)$ reveal medium and higher than \pm 0.5 reveal a strong correlation. Positive or negative $r$ values mean positive or negative correlations, respectively. 


\subsection{Results}

\subsection{1. shRNA IRS1 knocks down IRS1 mRNA levels in HEK293 cells}

To verify the knockdown efficiency of the shRNA against IRS1, we measured IRS1 and IRS2 mRNA expression in transfected HEK293. We used two constructs, expressing two different shRNA sequences (shIRS1A and shIRS1B) compared to plasmid control. We observed that expression of IRS1 but not IRS2 mRNA was significantly reduced by both sequences (Fig S5.2). The shRNAs were designed against rat, but they displayed 98\% homology with humans, so we could translate the results from human cells to rat IRS1.

5.3.2. AAV-shIRS1-EGFP injection in dorsal hippocampus alters spatial memory, especially in females, but not social memory, nor anxiety or depressive symptoms

AAV-shIRS1 and AAV control (Fig 5.1A) were generated in HEK293 cells with a titration of $1.02 \mathrm{E}+11 \mathrm{GC} / \mathrm{mL}$ and $4.94 \mathrm{E}+11 \mathrm{GC} / \mathrm{mL}$, respectively. AAV particles were bilaterally injected by stereotaxis in the dorsal HPC (AP $-4.36 \mathrm{~mm}, \pm$ ML $2.5 \mathrm{~mm}$, DV $-2.5 \mathrm{~mm}$ from Bregma) (Fig 5.1B). Control and shIRS1 injected animals were subjected to behavioural paradigms one month after viral particle injection (Fig 5.1C). Both paradigms evaluate the memory in rodents exploiting their innate exploratory preference for novel stimuli, the NOR test examines the capability of the animal to remember a familiar object. To quantify it, the DI is obtained by subtracting the time exploring the familiar object to the time exploring the novel one; divided by total exploring time. We observed that the shIRS1 injected females animals (DI; $0.24 \pm 0.13$ ) discriminate both objects significantly less than the AAV control injected animals (DI; $0.56 \pm 0.11$ ); whereas we did not see the same effect in males receiving shIRS1 (DI; $0.44 \pm 0.05$ ), compared to controls (DI; $0.47 \pm 0.06$ ) (Fig 5.2A). The data are represented by mean \pm SEM and analyzed using unpaired Student's t-test $(* p<0.05)$.

A

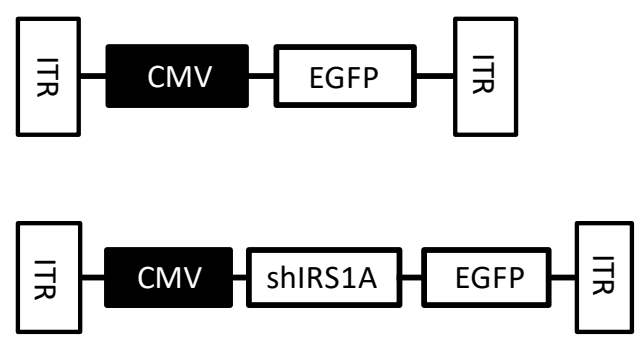

B

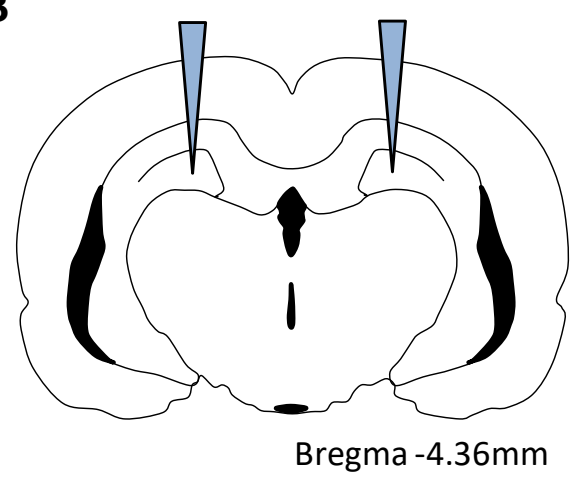

C

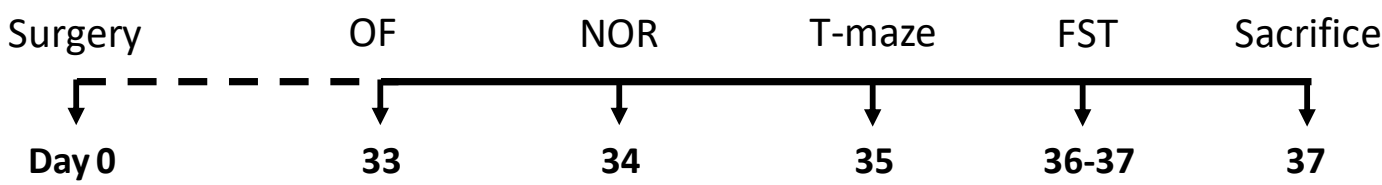

Fig. 5.1. Experiment design. Bilateral injection of the viral particle (AAV-CMV-GFP, 4.94E $+11 \mathrm{GC} / \mathrm{mL}$ or AAV-CMV-shIRS1-EGFP,1.02E + $10 \mathrm{GC} / \mathrm{mL}$ ) (A) into the dorsal hippocampus (AP $-4.36 \mathrm{~mm}, \mathrm{ML} \pm 2.5 \mathrm{~mm}, \mathrm{DV}-2.5 \mathrm{~mm}$ from bregma); $2 \mu \mathrm{L}$ of viral particle per hemisphere were injected (B). Behavioural tests started 33 days post-injection, and the animals were sacrificed at day $37(\mathrm{C})$.

In the T-maze test, we recorded the number of entries and the time exploring the novel arm. Females in the shIRS1 group showed reduced DI $(-0.03 \pm 0.10)$ in the time spent in novel arms compared to controls DI $(0.23 \pm 0.08)$ (Fig 5.2B). Similarly, in males, shIRS1-injected male rats 
had significantly lower DI value $(-0.00 \pm 0.04)$ than controls (DI; $0.16 \pm 0.07)$ in the time spent in the novel arm. Females showed significantly lower DI $(0.02 \pm 0.02)$ in the number of entries (Fig $5.2 \mathrm{C})$ to the novel arms compared to the control group DI $(0.14 \pm 0.06)$. Also, in males, the number of entries to the novel arm was lower (DI; $-0.02 \pm 0.03$ ) than controls (DI; $0.09 \pm 0.04$ ). These results suggested that the downregulation of IRS1 impairs the memory of the familiar arm, both in female and male rats. We evaluated anxiety by the latency to cross for the first time the open field and observed lower but not significantly different shIRS1 $(43.13 \pm 11.06)$ compared to control AAV (61.67 \pm 22.48$)$ injected females. Nor differences either in shIRS1 $(14.50 \pm 4.59)$ and control AAV (25.86 \pm 5.82 ) injected males (Fig 5.2D). Finally, we assessed depressive symptoms in the animals by the forced swimming test. As above, hippocampal injection of shIRS1 had no effect, since immobile time was equal between groups, control (111.20 \pm 9.15$)$ and shIRS1 (140.20 \pm 19.21) injected female rats and control $(157.80 \pm 15.17)$ and shIRS1 $(184.90 \pm 7.18)$ injected male rats (Fig 5.2E). Swimming time and climbing were not different either between groups (data not shown).
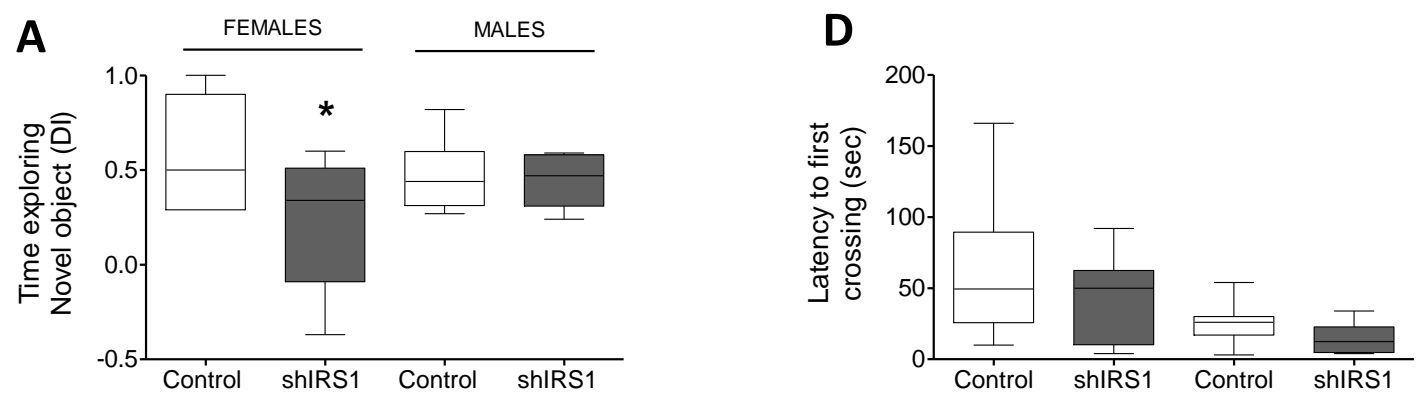

B

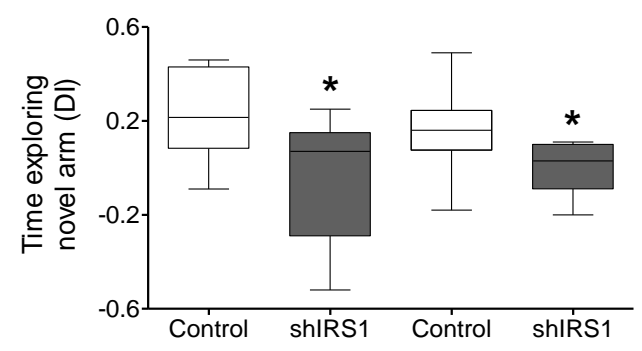

$\mathbf{E}$
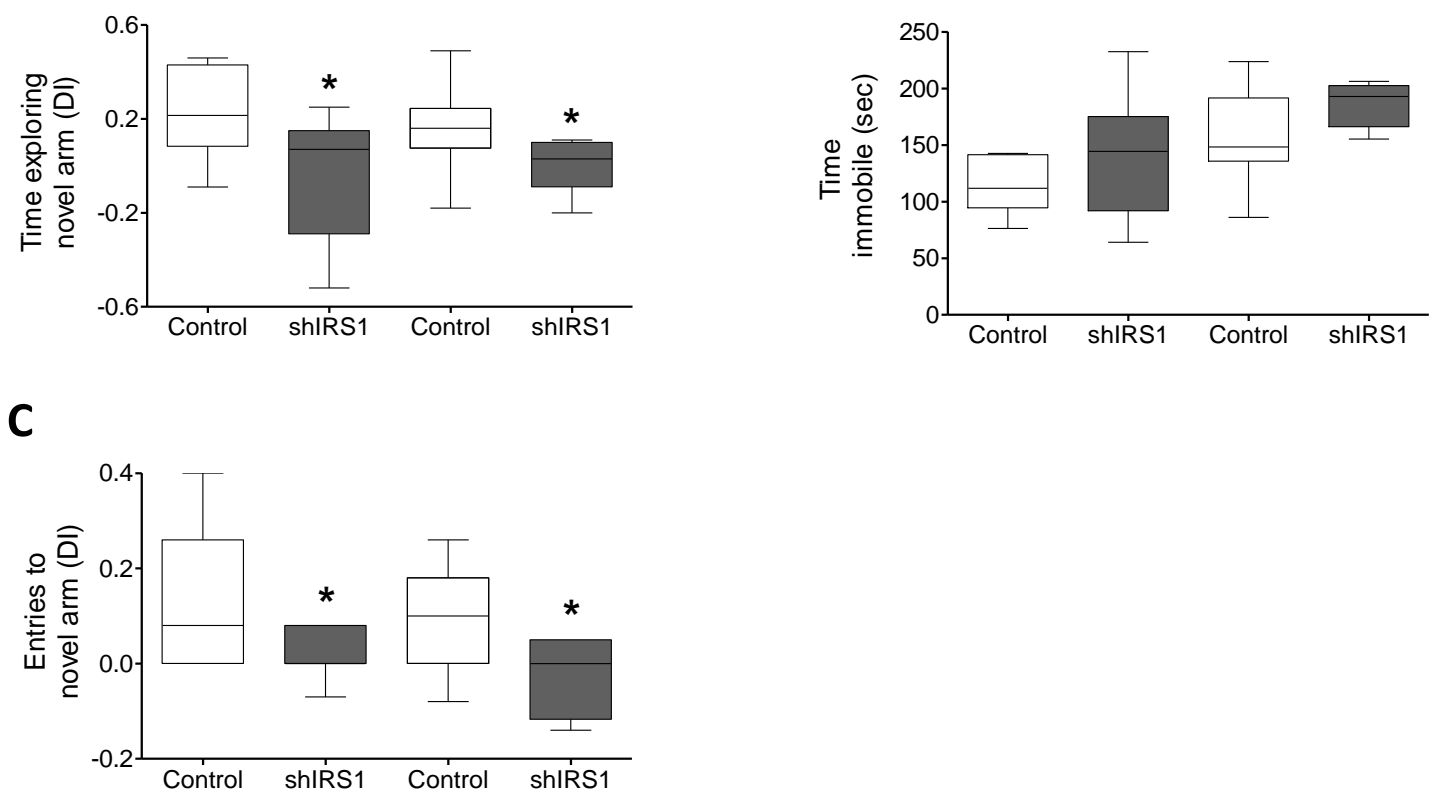

Fig. 5.2. AAV shIRS1 impairs spatial memory. Time exploring the novel object in the Novel Object Recognition test (A). Time exploring the novel arm (B) and the number of entries to the novel arm (C) in the T-maze test. Latency to cross the center of the arena for the first time in the Open Field test (D). Time spent immobile in the Forced Swim test (E). Data are expressed as discrimination index [(Time exploring novel - time exploring familiar)/total time exploring], presented as mean \pm whiskers at maximum and minimum $(\mathrm{n}=7-8$ per condition $)$ and analyzed using one-tailed Student t test $* p<0.05$. 


\subsubsection{AAV DJ8 tracks dorsal hippocampus efferent connections}

Dorsal HPC efferent projections reach MS and MM through the fimbria-fornix white track (Fig 5.3A). We inoculated AAV-CMV-shIRS1-EGFP and AAV-CMV-EGFP in the dorsal HPC. We observed fibers and cells in the DS, an area within the hippocampal formation (Fig 5.3B), and in the polymorphic area or hilus of the dentate gyrus (PoDG) (Fig 5.3C). As expected, we found fibers in the fornix (f) (Fig 5.3D) and consequently in MS (Fig 5.3E) and MM, specifically in the SuM (Fig 5.3F).
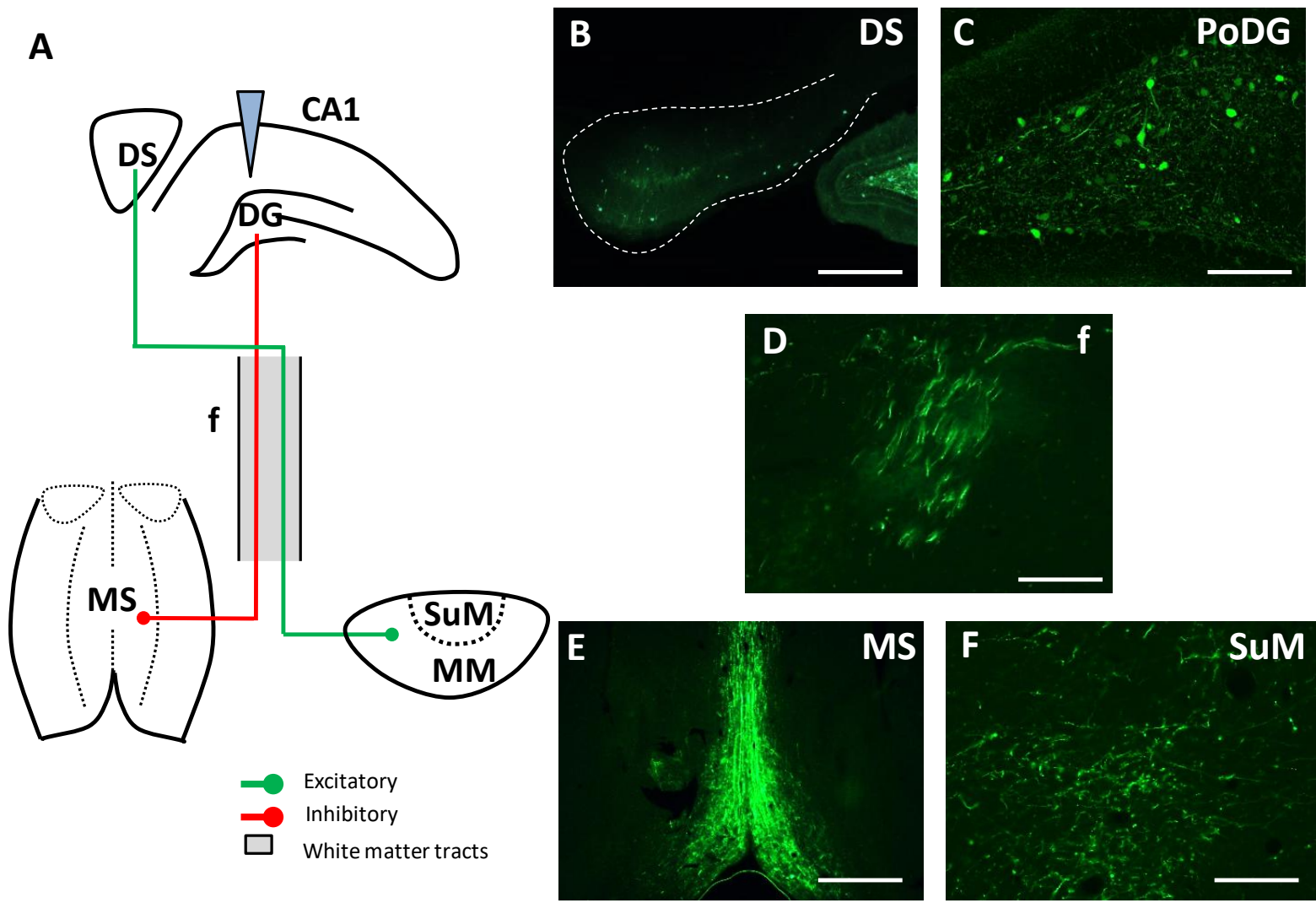

Fig. 5.3. AAV DJ8 traces dorsal hippocampus efferents. Schematic representation of the projections from the dorsal hippocampus, where the viral particle was inoculated (A). Representative images of 3 animals (per condition) and 3 slices each of neuronal somas in the dorsal subiculum (DS) (B), and the polymorphic layer of the dentate gyrus (PoDG) (C); AAVlabeled fibers in the medial septum (MS) (E) and supramammillary nucleus (SuM) (F) via fornix (f) (D). Scale bar $=200 \mu \mathrm{m}$ (B). Scale bar $=100 \mu \mathrm{m}$ (C-F).

\subsubsection{AAV-DJ8 labels dorsal specific hippocampal neurons}

AAV infected mostly neurons in the hilus of the DG, but not the granule layer (Fig 5.4). To a lesser extent, the AAV entered the CA1 (Fig S5.3). We observed that astrocytes were not infected by the AAV tropism (Fig S5.4). Diverse GABAergic cell types localize in the DG, so we performed immunostaining of neuronal markers to characterized AAV-infected neurons. We found that AAV labels co-localized with CR (Fig 5.4C), PV (Fig 5.4D), and SOM (Fig 5.4E) neurons. The quantification of the EGFP-cell marker double labelling revealed that $48.78 \% \pm 2.97$ of SOM cells and $42.88 \% \pm 3.75$ of PV had been infected with AAV, whereas the number of infected CR cells was slightly lower; $32.30 \% \pm 3.39$, suggesting that the SOM and PV neuronal activity has been more affected by IRS1 silencing (Fig 5.4B). 
A

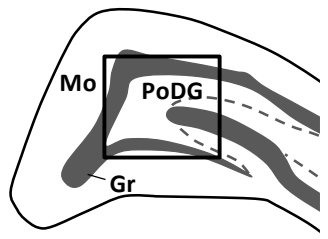

Bregma $-4.36 \mathrm{~mm}$

B

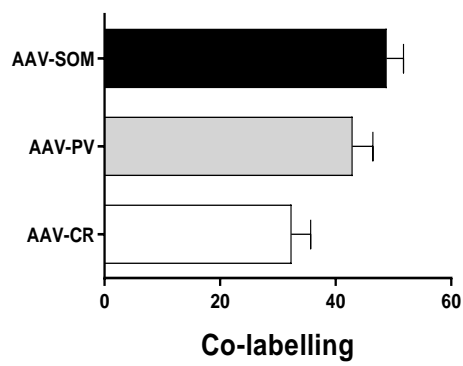

(\% of total marker positive cells)
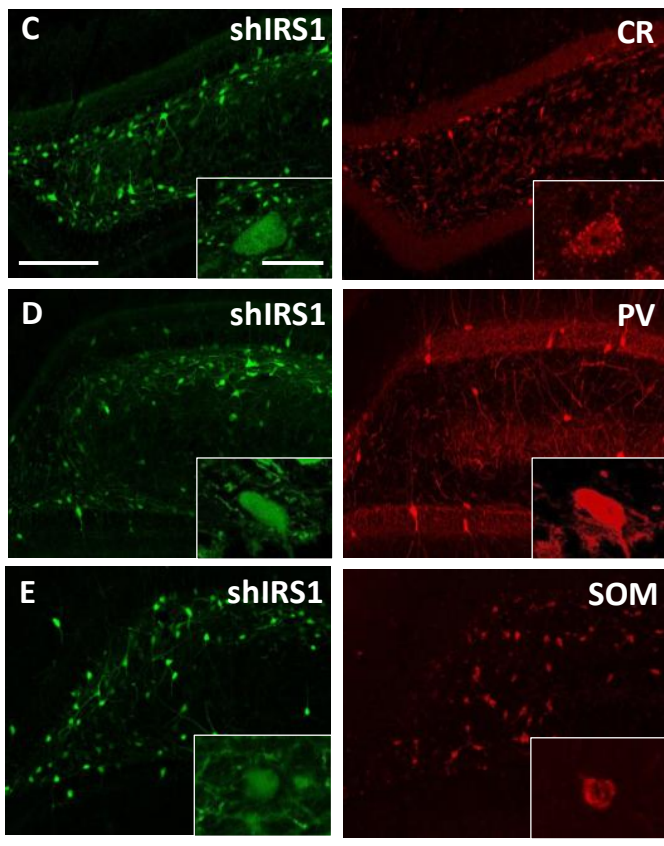

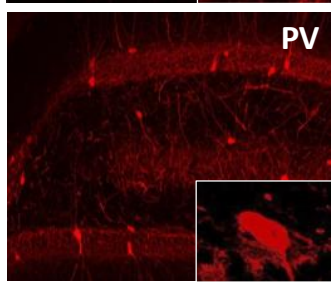

PV
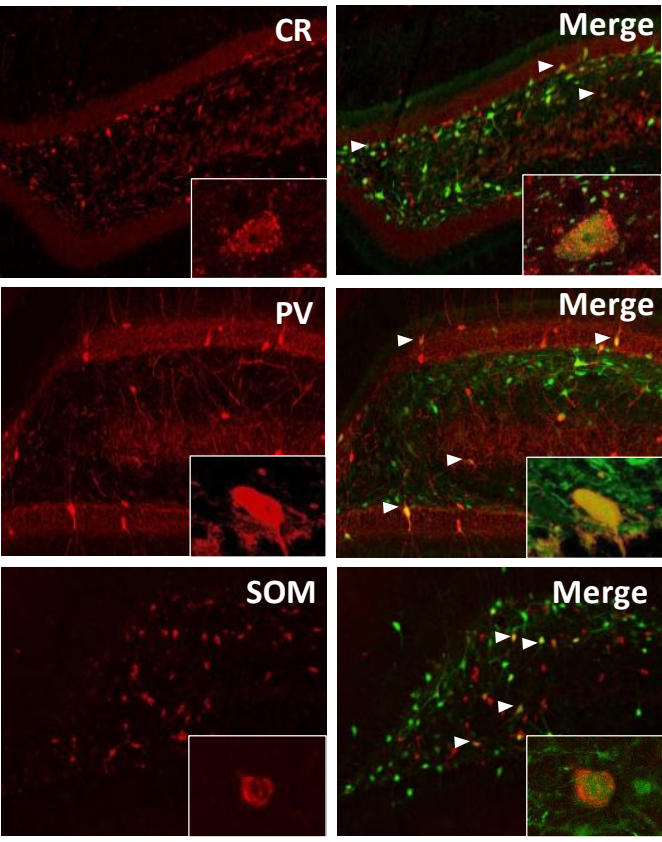

Fig. 5.4. AAV DJ8 labels dorsal specific hippocampal neurons. Schematic representation of the dentate gyrus of the dorsal hippocampus (A). Percentage of double-labelled neurons (EGFP + neuronal marker), relative to the total number of neuronal marker-positive cells. Data are represented as the mean $\pm \operatorname{SEM}(n=11)(B)$. Representative images of 4-6 animals (per condition) and 3 slices each of EGFP (green) co-localizing with calretinin (CR) (C): Parvalbumin (PV) (D); and Somatostatin (SOM) (E) positive neurons in the polymorphic area (PoDG) of the dentate gyrus. Scale bar $=100 \mu \mathrm{m}$. Scale bar $=20 \mu \mathrm{m}$ (insets).

\subsubsection{AAV DJ8 axons in the medial septum reach $P V$ and ChAT occupied areas}

We observed that MS was strongly innervated by AAV-labelled axons. The fibers seem to be restricted to the most medial part. To evaluate the type of neurons potentially regulated by the AAV-labelled projections, we carried out a series of immunolabelling analysis. We observed that AAV projections co-localized mostly within PV (Fig 5.5B) and ChAT (Fig 5.5C) areas. Also, sparse CB positive neurons fall within the axon labelled areas (Fig 5.5D). The field where CR neurons appear is completed devoid of EGFP fibres (Fig 5.5E). 
A

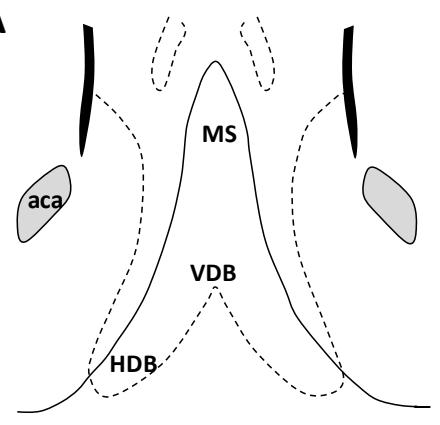

Bregma $1.08 \mathrm{~mm}$
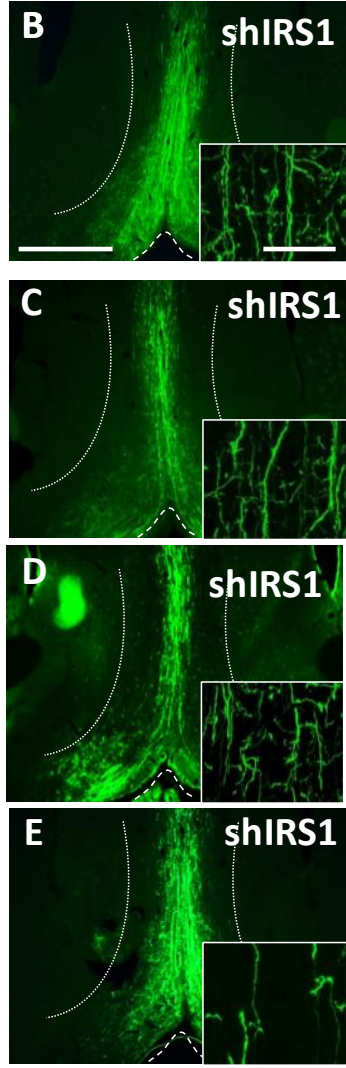
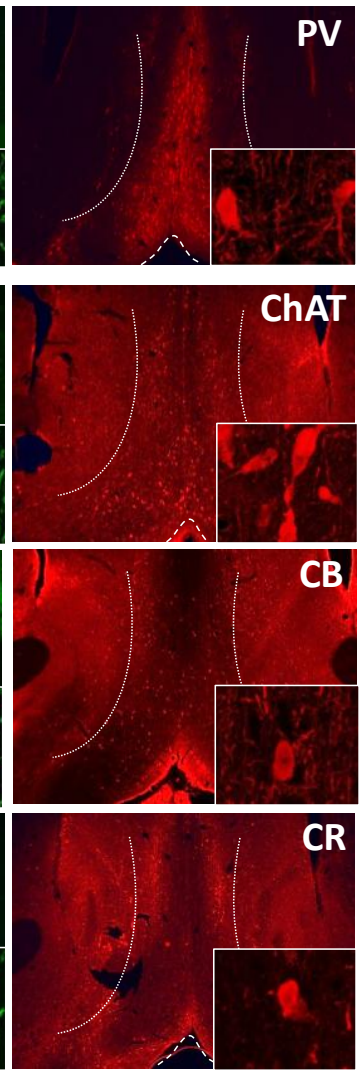
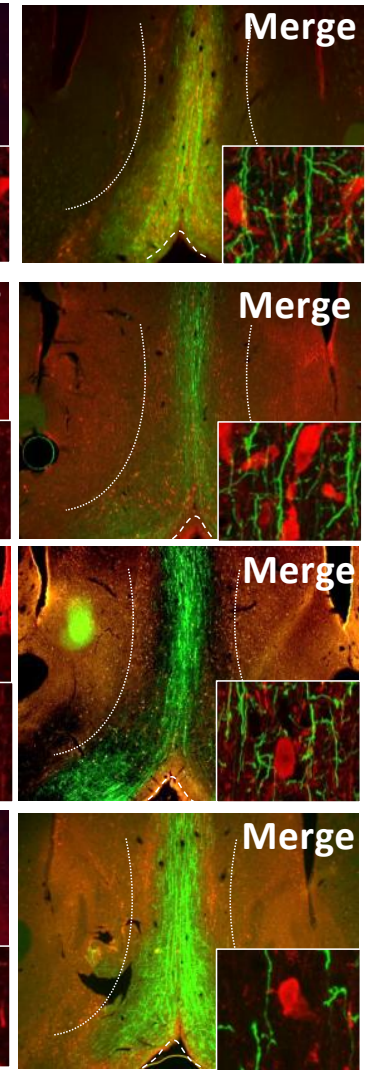

Fig. 5.5. AAV-labeled axons in the medial septum. Schematic representation of the medial septum (MS), vertical limb diagonal band (VDB), and horizontal limb diagonal band (HDB) (A). Representative images of 3 animals (per condition) and 3 slices each of showing AAV-infected neuron projections co-localized with parvalbumin (PV) (B), cholinergic (ChAT) (C) and calbindin (CB) area (D). We observed no co-localization of AAV green fibers with calretinin (CR) positive neurons field (E). Scale bar $=100 \mu \mathrm{m}$. Scale bar $=20 \mu \mathrm{m}$ (insets).

\subsubsection{AAV-shIRS1 results in lower expression of IRS1 in the dorsal hippocampus and correlates with behaviour}

To ascertain if AAV-shIRS1 corresponded to reduced IRS1 protein expression, double IRS1 immunofluorescence and EGFP expression were quantified and expressed in relation to total EGPF-positive neurons (Fig 5.6B). We observed that the AAV-shIRS1 resulted in a reduced number of double IRS1-EGFP neurons $(67.88 \% \pm 1.22)$, compared to control AAV $(80.28 \% \pm 1.9)$. This result is indicative of a knockdown of IRS1 expression in the dentate gyrus where the AAVshIRS1 was inoculated.

Moreover, to elucidate if the different degrees of reduced expression could account for the behavioural variability, we performed linear regression and applied the Pearson test, correlating the DI in time spent in the novel arms (T-maze) with the degree of IRS1 reduction (as shown in Fig 5.6B). We observed that the correlation between DI time in novel arms in the T-maze, was positive (Correlation DI time with shIRS1 $r=0.63$, and DI time vs control $(r=-0.15)$ (Fig S5.5). These results suggest that in AAV-shIRS1 subjects the degree of IRS1 reduction correlates better with the behavioural impairment than in control subjects. 
A

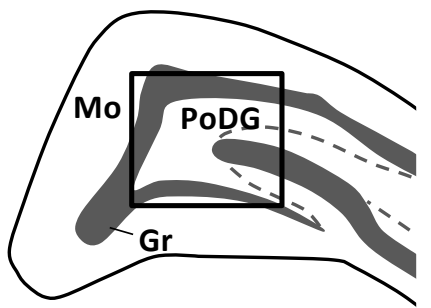

Bregma $-4.36 \mathrm{~mm}$
B

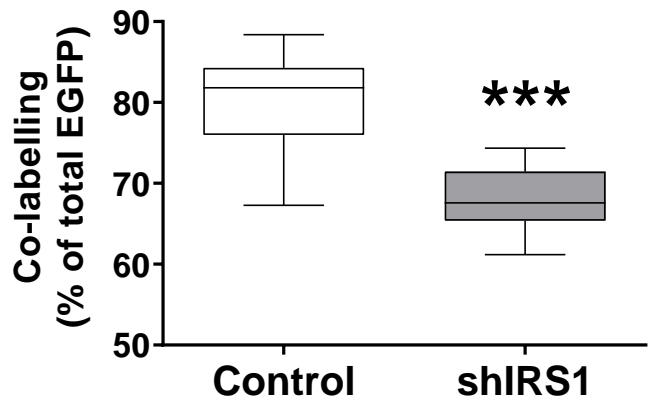

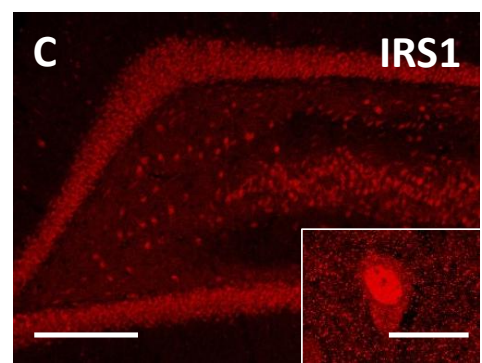
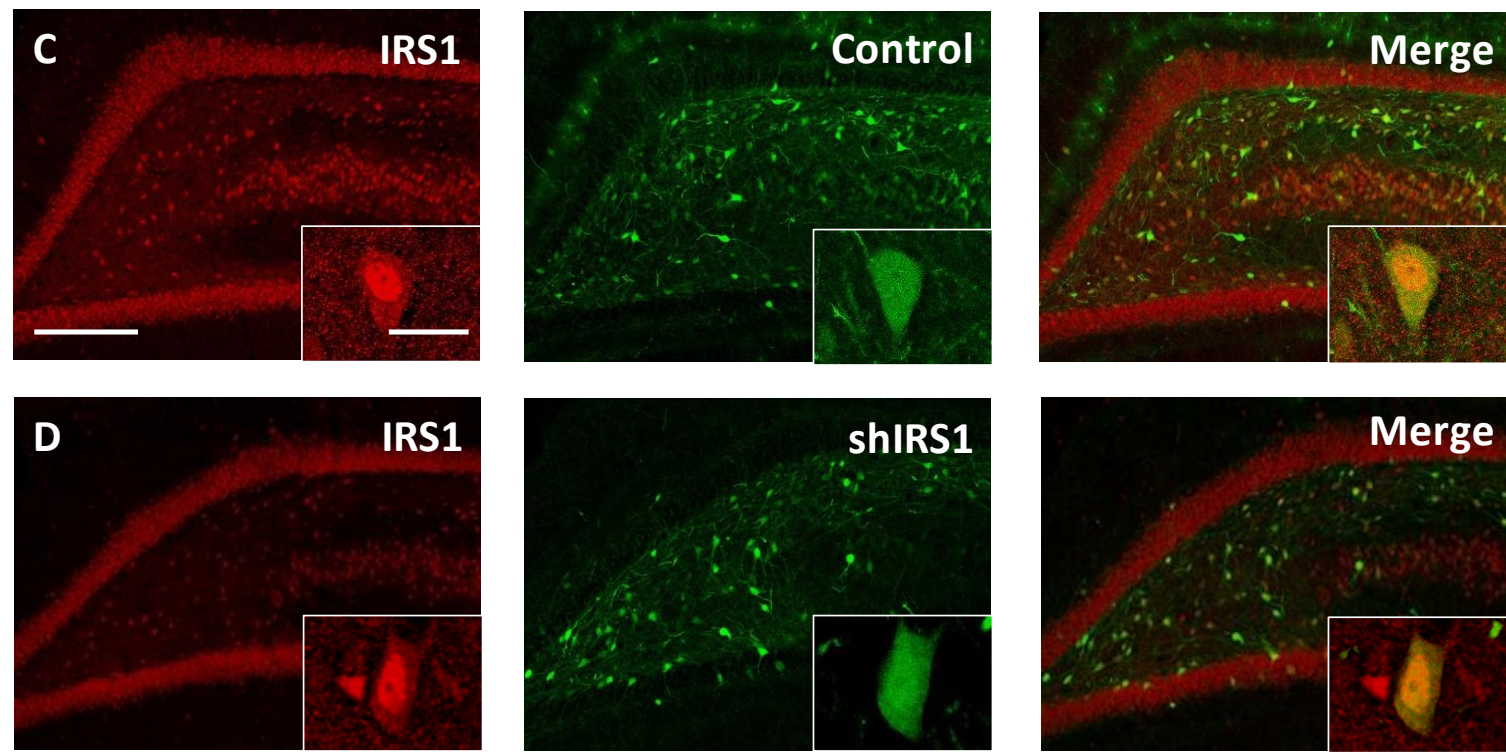

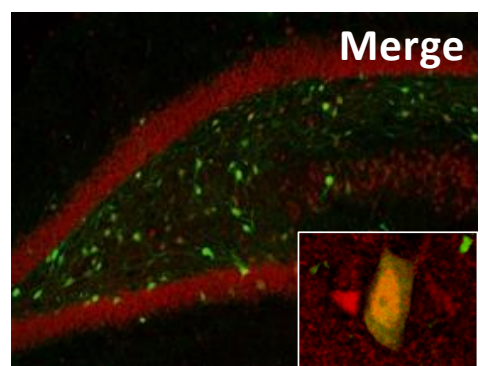

Fig. 5.6. Silencing effect of shIRS1 in the dorsal hippocampus. Schematic representation of the dentate gyrus of the dorsal hippocampus (A). Percentage of double-labeled neurons (IRS1 + EGFP), relative to the total number of EGFP-positive neurons (B). Representative images of IRS1, EGFP and co-localization of both labelling (merge) in the polymorphic area (PoDG) of control (C) and shIRS1 (D) animals. Data are represented as the mean \pm SEM $(n=10-11)$. Data were analyzed using one-tailed Student t test $* * * p<0.0001$. Scale bar $=100 \mu \mathrm{m}$. Scale bar $=20 \mu \mathrm{m}$ (insets).

\subsubsection{AAVshIRS1 infection results in lower synaptophysin positive labels compared to AAV control}

To explain the possible mechanism underlying the observed behavioural results we hypothesized that the reduction in IRS1 signalling could impair synaptic plasticity, based on previous data where insulin resistance reduced synaptophysin expression ${ }^{[340]}$ and reduced plasticity [341,342]. To evaluate the effect of shIRS1 in axons in the MS area (Fig 5.7A), we quantified synaptophysin labelling normalized by the green-labelled area. We observed that the number of synaptophysin-positive labels in axons was significantly lower in AAV-CMV-shIRS1-EGFP axons $(307.90 \pm 20.40)$ than in controls AAV-CMV-EGFP $(444.30 \pm 27.13)$ (Fig 5.7B). These results suggest that knocking down IRS1 reduces synaptic plasticity, as measured by synaptic vesicle proteins, used as an indirect marker of synaptic activity. Representative images are shown for AAV control (Fig 5.7C) and AAV-shIRS1 (Fig 5.7D). 
A

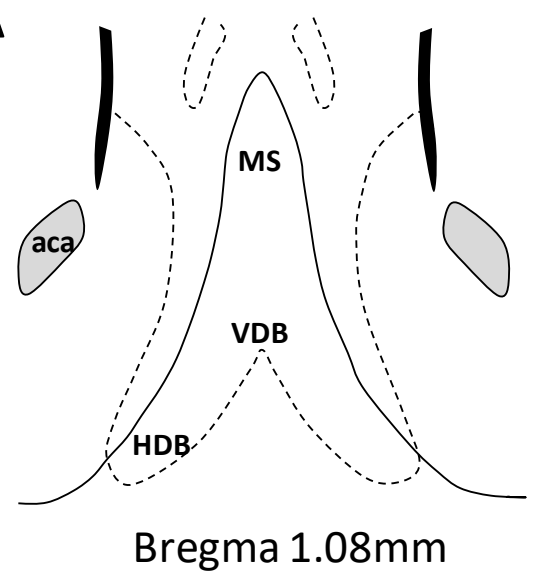

Bregma $1.08 \mathrm{~mm}$

\section{B}

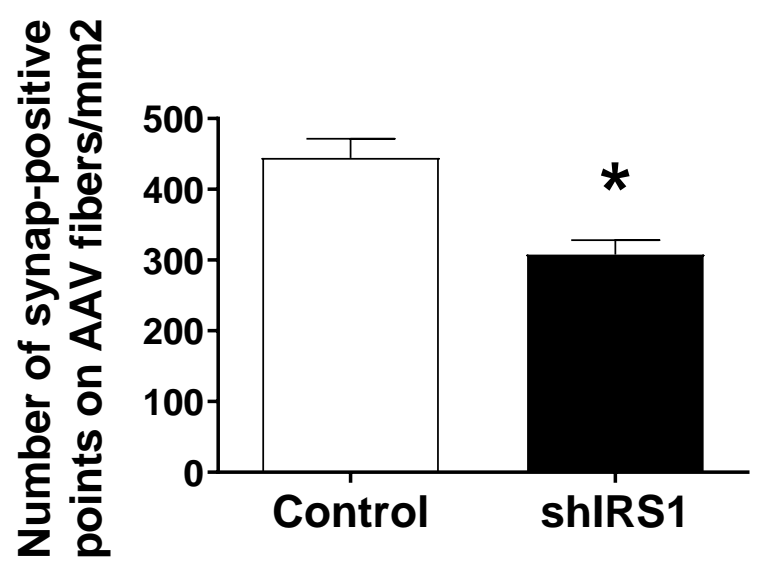

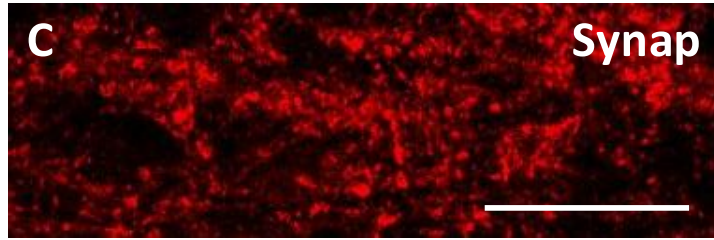
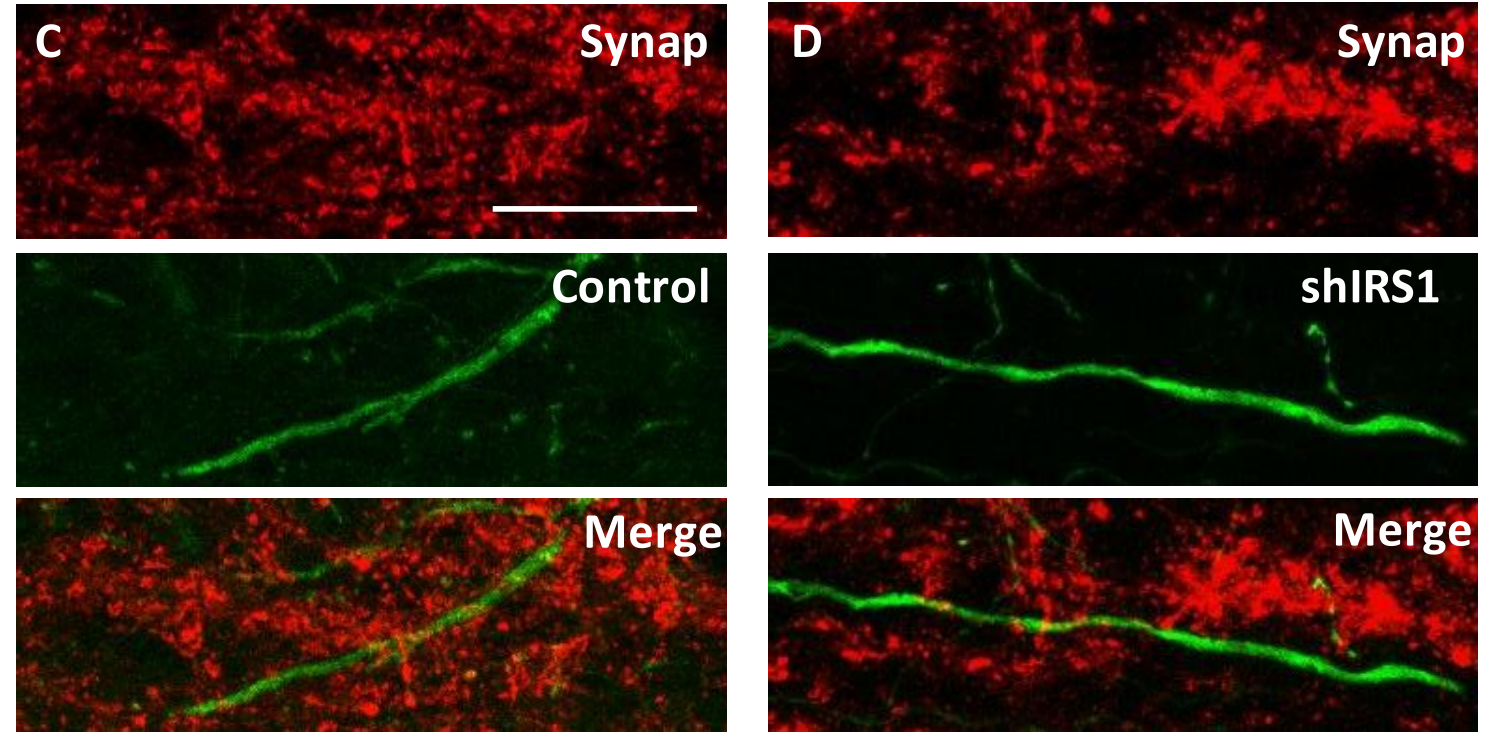

Fig. 5.7. AAV-CMV-shIRS1-EGFP inoculation in the dorsal hippocampus results in lower synaptophysin positive labels in fibers reaching medial septum. Schematic representation of the medial septum (A). The number of synaptophysin (Synap) positive signals on the EGFP-labeled fibers (B). Representative images of synaptophysin co-localization with AAV control (C) and EGFP-shIRS1 (D) fibers. Data are represented as the mean \pm SEM of the number of synaptophysin contacts with the green AAV labelling per $\mathrm{mm} 2$ of the fiber $(\mathrm{n}=3-4 ; 10$ fibers per animal). Data were analyzed using one-tailed Mann Whitney t test $* p<0.05$. Scale bar $=10 \mu \mathrm{m}$. 


\subsection{Discussion}

In our study, we show that infection of shIRS1 using an AAV2/DJ8 derived virus inoculated in the hippocampal area affects hippocampal memory formation in female and male rats. It does not alter anxiety parameters in any sex. Loss of spatial memory is one of the first symptoms in AD, caused by progressive loss of neuronal circuitries. Diabetes, dyslipidemia, and cardiovascular factors have been demonstrated to play a role in degenerative diseases like $\mathrm{AD}$ and frontotemporal dementias ${ }^{[343]}$. These pathologies are linked to brain insulin resistance, which triggers cognitive decline and $\mathrm{AD}{ }^{[344]}$. Most research addressing insulin resistance in the brain assumes that neuroinflammation causes insulin resistance, and this, in turn, aggravates neuronal damage, stimulating the neuroinflammatory process. Although neuroinflammation is a general process affecting the whole brain, in AD patients one of the first clinical symptoms is the loss of spatial memory. Thus, it is likely that only particular circuitries involved in this memory are affected at the early stages of the disease before massive neuronal death occurs. Major hippocampal circuitries like the septo-hippocampal connections ${ }^{[181]}$ and the circuit of Papez ${ }^{[187]}$ are crucial for hippocampal-dependent memory formation.

In this study, we made use of an AAV carrying a shRNA targeting IRS1. We confirmed mRNA downregulation by PCR in vitro studies and reduced protein expression in vivo by immunofluorescence. We also confirmed that the shIRS1 did not affect significantly IRS2 expression, so the knocking down is specific for this isoform. Most studies in the literature have focused on the IRS1 inactivation due to serine phosphorylation. Serine residues are phosphorylated by inflammatory signals. In this study we have used shIRS 1 to reduce the expression of IRS1, thus IRS1 signalling activity is reduced. This study is not a knockout, so a residual activity is present, and there are no compensations either which may alter the specific effect of an IRS1 reduced expression. Importantly, we wanted to isolate the IRS1 effect, since inflammatory processes affect several pathways, one of the IRS1, we aimed to examine the IRS1 contribution in discrete neuronal pathways in the absence of neuroinflammation. This may indicate a way to improve brain health in human pathologies where IRS1 signalling is affected.

Females rats inoculated with the AAV-CMV-shIRS1-EGFP performed significantly worse in the NOR test, compared to controls inoculated with AAV-CMV-EGFP. The NOR test evaluates the subject memory by measuring the recognition of familiar objects. When the animal does not remember having explored an object, it will explore both equally, as they are both equally new to the subject. Unlike female subjects, males inoculated with shIRS1 remember the familiar object, and they behaved similarly to control injected males. This result suggests that the female brain may display a higher susceptibility to insulin resistance, this would agree with the observation that AD is more prevalent amongst females ${ }^{[345]}$. In humans, $\mathrm{AD}$ is reported to be more prevalent in women than in men ${ }^{[346]}$. Reports indicate that around $70 \%$ of AD patients are women in the United States ${ }^{[347,348]}$ and in Europe ${ }^{[349]}$. Several hypotheses have been proposed to explain that fact. The most evident is that women have a longer life span than men, but even after normalizing to it, other biological factors have been considered. Amongst others, female higher sensitivity to the presence of one APOE 4 allele has been proposed ${ }^{[350,351]}$, since this isoform increases the probability to suffer from $\mathrm{AD}^{[352]}$. APOE is the major lipid transporting molecule in the brain and the isoform APOE 4 has less capacity to shuttle lipids than the others, thus, given the importance of cholesterol in neuronal function ${ }^{[353]}$, cholesterol dysregulation is expected to contribute to degenerative pathologies ${ }^{[354]}$. Because dyslipidemia and insulin resistance act in a vicious cycle to promote pathologic processes contributing to degenerative diseases; under the light of our results, we proposed that females, even with normal estrogens levels (in this study female rats were not ovariectomized) may be more susceptible to insulin resistance (due to reduced IRS1 function) than male. We hypothesize that this could be a major contributing factor to the higher prevalence of $\mathrm{AD}$ in women; however, further studies are required to unveil the underlying mechanism to this effect.

The T-maze evaluates the spatial navigation capability. Exploration of novel spaces is a hallmark of rodent, and as expected, female and male subjects inoculated with control AAV explored the new arm longer time than the familiar one. Both females and males injected with shIRS1 failed to discriminate between the maze novel and familiar arms, indicating that they do not 
remember the arm previously visited. These results agree with former studies reporting that IR silencing using a lentiviral derived particle inoculated into the HPC impaired spatial memory ${ }^{[355]}$. Importantly, in this task, we found no difference between male and female subjects. One possible explanation for this result is that the T-maze can be considered a more difficult task than the NOR task, it also measures navigation and NOR evaluates recognition. Therefore, we hypothesize that the NOR test may more sensitive to smaller neurological deficiencies ${ }^{[118]}$.

We observed no differences in anxiety-like behaviours. This may be in contrast with previous reports indicating that hippocampus-septal connections may regulate anxiety ${ }^{[356,357]}$. Because the circuit targeted with the AAV DJ8 virus has been proved very specific, we consider that we have only affected the hippocampal processing of spatial memories.

In this study, AAV DJ8 inoculated in the hippocampal area is mostly found in neurons within the PoDG or hilus of the DG and to a lesser extent in the DS. Sparse neurons were found in the CA1 regions (Fig S5.3). We observed abundant fibers via the fimbria-fornix pathway. This pathway is a white matter tract that contains hippocampal efferents (to MS and MM) and afferents (from septal cholinergic projections ${ }^{[358]}$ ). Consistent with hippocampal efferent we observed abundant fibers through the fornix. Following Papez circuitry, we also found fibers in the MM. Consistently, no thalamic staining was observed (data not shown). In addition, we found extensive green fibers in the MS. Traditionally, MS has been considered to receive projections from CA1 GABAergic SOM neurons ${ }^{[359]}$. However, long-range projection interneurons have been localized in the DG hilus innervating the MS ${ }^{[360]}$. Recently, these DG long-range projection interneurons have been characterized as a new type of SOM interneuron and named as hilar interneurons with long axons (HIL). HIL neurons co-exist with hilar SOM interneurons (the SOM HIPP neurons) ${ }^{[180]}$. Long axon SOM neurons provide regulation to both nearby PV interneurons and display collaterals projecting to the MS, where they contact ChAT and PV neurons ${ }^{[180]}$.

We cannot rule out the effect of IRS1 silencing in glutamatergic neurons in the HPC. However, since we have seen a high proportion of AVV infection in the hilus of the dentate gyrus, where most neurons are GABAergic, for clarity, we decided to focus this study on the GABAergic connections. This rationale is backed up by the strong green labelling we observed in the PV area within the MS, which corresponds to hippocampal SOM neurons projecting to the septal area. This, by no means, suggests that it is the only mechanism underlying the IRS role in neuronal activity. To better understand the role of IRS1 in Glutamatergic, different AAV serotypes may improve infection in the glutamatergic type of neurons.

We immunocharacterized the neurons within the DG infected with AAVDJ8 and confirmed the co-labelling with the SOM antibody. Moreover, AAV fibers were found where the PV and ChAT neurons are in the MS ${ }^{[180]}$, therefore, we assume that our AAV can be affecting both types of hilar SOM, the short and long projecting neurons. Both types of hilar SOM interneurons play an important role in regulating hippocampal function. The DG is the first phase of the classical trisynaptic circuit ${ }^{[361,362]}$, defined as the circuit starting from the EC to the CA3 pyramidal neurons, and from these to the CA1. This circuit is crucial for the formation of new memories ${ }^{[363,364]}$. Thus, information coming from the entorhinal is regulated with precise timing by the strong inhibitions within the DG, this modulates the output of granule cells to CA3, where spatial memory forms a spatial pattern. HIPP provides dendritic inhibition controlling the entrance of spatial information from the EC to the DG. In addition, the inhibition mediated by HIL cells is proposed to contribute to the temporal coordination of MS and local rhythmic DG activity ${ }^{[180]}$. Since we do not see soma labeling in the MS, we assume that AAV only travels anterogradely through the fimbria-fornix to MS, therefore, we assume that MS neuronal activity is affected via hilar SOM long projections.

We found that axons from the HPC innervating MS (the long projecting SOM) have reduced synaptophysin labelling, indicative of reduced synaptic plasticity. Even though we cannot rule out other mechanisms, this result illustrates that the reduction in IRS1 function impairs long projecting SOM neuronal plasticity with behavioural consequences. Synaptophysin labelling is a marker of presynaptic activity; together with postsynaptic markers it is considered a measure of the circuit 
synaptic plasticity. Presynaptic alterations of synaptophysin labelling have been reported as an early event in $\mathrm{AD}^{[365]}$.

We speculated that IRS1 silencing may alter neuronal presynaptic activity, and even if we cannot rule out an alteration at the postsynaptic level, we focused on the connection hippocampusseptum, and we used synaptophysin labelling as a presynaptic marker of synaptic activity. Indeed, we found that synaptophysin staining was reduced in shIRS1 infected neurons compared to controls. The mechanism underlying this observation can be a reduced IRS1-PI3-AKT pathway, that regulates GLUT4 translocation to the membrane ${ }^{[366]}$, which would hamper glucose availability at the nerve terminals, thus impairing synaptic activity ${ }^{[367]}$. The long-term modulation of synaptic activity is achieved (amongst others) by regulating the expression of several neurotransmitter vesicle proteins. This has been shown with a high-fat-diet model that reduced IGF-1 and synaptophysin levels. These were recovered after IGF-1 signalling activation ${ }^{[368]}$. Also, BDNF has been shown to modulate synaptic plasticity by regulating synaptophysin expression ${ }^{[369,370]}$.

IRS1 has been mostly studied in the context of insulin and IGF-1 signalling, however, given their scaffolding function it is plausible that IRS1 mediate several neurotrophic factors, including BDNF ${ }^{[328]}$. This would agree with the fact that even a small reduction in the IRS1 expression function has resulted in a behavioural defect.

In addition to SOM, we found co-labelling with GABAergic PV interneurons, and to a lesser extent with CR neurons. Fast spiking PV interneurons regulate network oscillation and synchrony ${ }^{[371]}$. Previous reports demonstrate that PV dysregulation in the DG affects memory and anxiety ${ }^{[372]}$. However, in our present work, we do not see alterations in anxiety nor social behaviour or depressive symptoms, but specifically spatial memory.

Under the light of our results, we hypothesized that IRS1 signalling is required for both SOM and PV inhibitory action, by reducing synaptic plasticity. We believe that we have affected two pathways by the AAV infection; on one hand, there may be disinhibition of the information coming from the EC, hampering the formation of a clear spatial pattern in CA1. On the other hand, there may be disinhibition of PV and ChAT neurons in the MS, which in turn may increase PV and cholinergic action back into the HPC, this dysregulation can thus impair the excitatory/inhibitory balance.

Moreover, the Papez circuitry is essential to memory formation ${ }^{[373]}$. Papez circuitry starts in the subiculum, a part of the hippocampal formation. Evidence supports that the sequential pathway from the subiculum to the MM via the fornix, and from there to the anterior thalamus, is required for spatial memory in rats ${ }^{[374]}$ and humans ${ }^{[375]}$. Moreover, this circuitry is found severely affected in $\mathrm{AD}$ patients ${ }^{[376]}$. Because viruses do not "jump" to adjacent neurons, the effect of the IRS1 silencing is confined to the hippocampal projection to the MM via the fornix. Some reports that have specifically disrupted this efferent projection indicate that it has only a mild effect on memory formation ${ }^{[377]}$. Other studies, while emphasizing MM function in memory formation ${ }^{[378]}$, indicate that other projections from brain stem nucleus (e.g. the tegmental nuclei of Gudden), and not the hippocampal formation, are critical for sustaining MM function ${ }^{[378,379]}$. Therefore, taking together these findings, we may assume that the effect we have observed disrupting memory formation in rats inoculated with AAV shIRS1 is most likely due to IRS1 knockdown in hilar DG GABAergic neurons, modulating the incoming flow of information from the EC and projections to the MS.

Taking together all these findings, our works supports that IRS1 signalling in GABAergic hippocampal neurons, both interneurons, and MS projecting neurons is required for forming spatial memories. In this model, we mimic insulin resistance by silencing IRS1 in an otherwise healthy brain (i.e no inflammatory ambiance). In neurodegenerative diseases, inflammatory conditions induce insulin resistance, and concomitantly, loss of cognitive function. Our results indicate that insulin resistance, in the absence of inflammatory conditions, is sufficient to cause memory impairments, suggesting that this could be at the very onset of neurodegenerative diseases. 


\section{6}

Chapter 4

\section{TARGETING NEUROINFLAMMATION IN A MICE MODEL OF ADHD WITH ALTERED PAIN SENSITIVITY. A PILOT STUDY}




\subsection{Introduction}

ADHD is one of the most common neurodevelopmental disorders in children and adolescent worldwide (incidence $8-12 \%)^{[380]}$. Main symptoms are impulsivity, hyperactivity, and problems with sustained attention ${ }^{[381]}$. ADHD often coexist with other neurodevelopmental alterations (i.e. autism spectrum disorder); and also with anxiety and depression ${ }^{[381]}$. Currently, pharmacological treatment implies psychostimulants chronic administration (e.g. methylphenidate) which raises reasonable concerns regarding its potential dangerous long-term effects ${ }^{[382,383]}$ including addiction ${ }^{[384]}$; and anxiety ${ }^{[385]}$. Therefore, it is of fundamental interest to investigate the etiology of the disease to develop new therapeutic strategies. Strong evidence has indicated that dopaminergic system dysfunction stands at the onset of ADHD ${ }^{[386]}$. To investigate the pathophysiology of ADHD, several animal models have been developed ${ }^{[387]}$. Among them, neonatal lesion with 6hydroxydopamine (6-OHDA) has prevailed since it is specifically toxic to central dopaminergic pathways. This model simulates key hallmarks of the human disease including hyperactivity and its paradoxical response to psychostimulants ${ }^{[388,389]}$.

Increased inflammation during early neurodevelopment is suspected as a risk factor aggravating and/or triggering ADHD symptoms ${ }^{[390]}$, although no mechanism has been proposed, yet. Epidemiological studies including metanalyses reveal that patients with ADHD are more likely to suffer asthma, allergic rhinitis, atopic dermatitis, and allergic conjunctivitis (well-known inflammatory conditions) than control subjects ${ }^{[391-393]}$. Moreover, maternal inflammatory status can prompt the occurrence of ADHD in the offspring; one prospective study (more than 23000 patients) revealed that a maternal history of autoimmune disease was associated with an increased risk of ADHD ${ }^{[394]}$. Yet, studies evaluating serum inflammatory markers in patients have not reached strong conclusions ${ }^{[395]}$. Other studies have shown certain IL-6 and TNF $\alpha$ gene polymorphisms associated to ADHD patients ${ }^{[396]}$. Furthermore, it has been found that oxidative stress markers levels, such as malondialdehyde (MDA) and nitric oxide (NO), were increased in ADHD children ${ }^{[397]}$ and adults ${ }^{[398,399]}$. That neuroinflammation biomarkers in ADHD children were associated to comorbid anxiety, not to "pure" ADHD ${ }^{[400]}$. For this subset of patients, environmental stress (e.g. confinement) will aggravate ADHD symptoms. Thus, is urgent to elucidate the relationship between neuroinflammation and comorbidities in ADHD patients.

Given the previous work we have carried out with ABA, where we have shown that oral $\mathrm{ABA}$ administration can ameliorate neuroinflammation-induced brain alterations, we proposed that ABA could be a good candidate to treat psychiatric conditions of neuroinflammatory etiology. Furthermore, in human SH-SY5Y cell culture, ABA protected DA neuroblastoma cells from damage and death induced by 6-OHDA neurotoxicity ${ }^{[401]}$.

Attention and pain are intimately related; thus, attention modulates pain perception as demonstrated by studies where subjects engaged in a task requiring attention perceived less pain to same stimulus than subjects not doing such task ${ }^{[402]}$. Conversely, chronic pain reduces attention span ${ }^{[403]}$. In humans, recent evidence indicates that ADHD increases pain perception in adults ${ }^{[404,405]}$ and the prevalence of generalized pain is higher in ADHD patients (up to 80\%) compared to control population $(17 \%)^{[406]}$. Interestingly, methylphenidate treatment for ADHD can partially reduce nociception, in adults ${ }^{[407]}$. Also, children with debilitating chronic pain present higher risk of having comorbid neurodevelopmental disorders, including autistic traits and symptoms consistent with $\mathrm{ADHD}^{[408]}$.

Interestingly, neuroanatomical studies confirm that attentional processes and pain transmission use identical neural networks. Thus, it is plausible that neuroinflammation affecting synaptic function in specific brain areas, is a risk factor for ADHD symptoms and for the altered pain sensitivity.

The available data shows the importance of neuroinflammatory mechanisms in both altered pain sensitivity and ADHD. This pilot study represents preliminary data aiming to elucidate whether ABA treatment in a preclinical model of ADHD can ameliorate ADHD symptoms and 
altered pain sensitivity. We hypothesized that this effect would be due to reduced inflammation within relevant brain areas, such as ACC-insula.

\subsection{Materials and methods}

The workflow is depicted in Fig. 6.1.

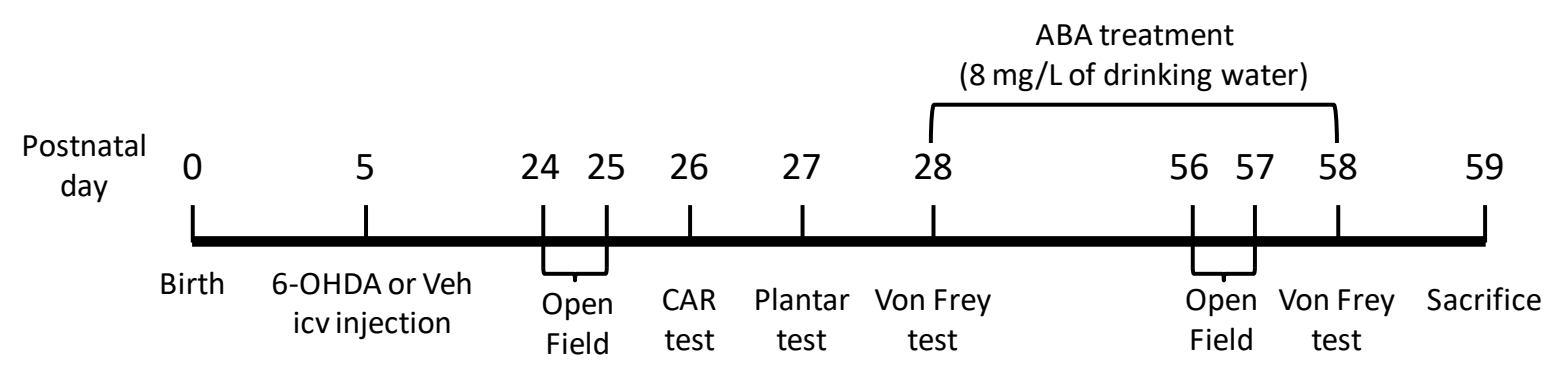

Fig. 6.1. Schedule of the experiment design. 6-OHDA icv (intracerebroventricular) injection was performed at P5. ABA administration $(8 \mathrm{mg} / \mathrm{L}$ in the drinking water) started in $\mathrm{P} 28$ and finished in P58. Behavioral tests were carried out between P24-P28 and two paradigms (Open field and Von Frey test) were repeated after 4 weeks of ABA treatment. Animals were sacrificed at P59.

\section{Animals and surgical procedures}

Eighteen C57BL/6 mice (9 females and 9 males) were bred and kept in the animal facility of Centre Paul Broca-Nouvelle Aquitaine (University of Bordeaux). The procedures followed directive 86/609/EEC of the European Community on the protection of animals used for experimental and other scientific purposes. The experiments were approved by the Ethics Committee of the University of Bordeaux (approval number APAFIS-27681). Once the mice were born, they were housed with their mothers and maintained on a $12 \mathrm{~h}: 12 \mathrm{~h} \mathrm{light-dark} \mathrm{cycle} \mathrm{(lights}$ on at $7 \mathrm{am}$ ) with food and water ad libitum.

At postnatal day 5 (P5), pups received an icv injection of 6-OHDA hydrobromide (SigmaAldrich, France) or vehicle as described in Bouchatta et al. $2018^{[388]}$. Thirty minutes before surgery, mice were injected with desipramine hydrochloride pretreatment $(20 \mathrm{mg} / \mathrm{kg} \mathrm{sc}$; SigmaAldrich, France), an inhibitor of the noradrenergic re-uptake, to protect noradrenergic system against the 6-OHDA-induced degeneration. Mice were anesthetized with isoflurane $4 \%$ and maintained with isoflurane $2 \%$ during surgery. Stereotaxic coordinates used to infuse $25 \mu \mathrm{g}$ of 6 OHDA dissolved in $3 \mu \mathrm{l}$ ascorbic acid $0.1 \%$ or vehicle into one of the lateral ventricles were AP -2 $\mathrm{mm}, \pm$ ML $0.6 \mathrm{~mm}, \mathrm{DV}-1.3 \mathrm{~mm}$ from bregma. After weaning (P21), mice were divided per sex in groups of 2-4 animals to reduce stress due to social isolation.

\section{Abscisic acid treatment}

At P28, 6-OHDA mice were divided randomly into two experimental groups per sex: animals that received ABA (Sigma-Aldrich, Spain) in the drinking water $(8 \mathrm{mg} / \mathrm{L}$ ) and animals that received vehicle (VEH). Due to the limited number of animals, the Sham group of both sexes received VEH in the drinking water. All groups were fed ad libitum for 4 weeks. The body weight and water intake were monitored once a week per cage.

\section{Behavioural procedures}

All the behavioural paradigms were carried out at P24. Open field and Von Frey test were repeated at the end of $\mathrm{ABA}$ treatment (P56-58). One week before the start of the behavioural testing, daily handling to all the animals was carried out. In all the procedures, mice were habituated to the testing room 30 minutes before performing each behavioural paradigm. 
Behavioural tests were conducted during the day with a dim light and at the same time zone to avoid activity differences caused by the light-dark cycle.

Open Field; The open field apparatus consisted of a grey PVC box $(40 \times 40 \times 30 \mathrm{~cm})$ combined with infrared sensors. Mice were placed in the centre of the open field and allowed to freely explore the arena for $10 \mathrm{~min}$. The test was divided in two days to reduce the effect of excitation caused by novelty on the spontaneous locomotor activity. The box was cleaned with a 30 $\%$ ethanol solution between animals to eliminate olfactory cues. The velocity $(\mathrm{cm} / \mathrm{s})$ and the distance travelled $(\mathrm{cm})$ were quantified. Animals were recorded using the EthoVision XT video tracking system (Noldus, Wageningen, Netherlands).

Cliff avoidance reaction (CAR) test; CAR assessment was performed placing mice over a round plastic platform $(20 \mathrm{~cm}$ diameter, $50 \mathrm{~cm}$ height). Animals were gently placed over the paradigm with the forelimbs close to the edge and the latency to the first fall was registered. Then, mice were immediately placed back on the platform and the number of falls was recorded for 60 minutes. If one animal did not fall, it was not removed from the platform during the same duration of time. The paradigm was cleaned with a $30 \%$ ethanol solution between animals. Usually, animals tend to avoid falling from a high surface, thus when a mouse fell from the platform it is showing an impaired CAR. Animals were recorded using the MediaRecorder video software (Noldus, Wageningen, Netherlands).

Plantar test (Hargreaves apparatus); The thermal nociceptive response was assessed using the plantar test paradigm (Ugo Basile, Varese, Italy). Each mouse was placed inside individual cages $(10 \times 10 \times 14 \mathrm{~cm})$ with a glass pane floor where animals could freely move. During the first 30 minutes, mice were habituated to the environment. Using an infrared (IRed) generator, ventral surface of left and right hind paws was stimulated with an IRed intensity of 50. The cut off time for finishing the IRed stimulation was set at 7 seconds. The latency to the paw withdrawal was recorded and 5 measurements per paw were done with an interval of 2 minutes between measurements. The individual cages and all the set up were cleaned with a $30 \%$ ethanol solution between animals.

Von Frey test; The mechanical nociceptive response was assessed using the Von Frey paradigm (Ugo Basile, Varese, Italy). Each mouse was placed inside individual cages $(10 \times 10 \times 14$ $\mathrm{cm}$ ) with a mesh floor where animals could freely move. During the first 20 minutes, mice were habituated to the environment. The ventral surface of left and right hind paws was stimulated with calibrated Von Frey filaments. The strength of filament application was considered enough when it buckled. If there was paw withdrawal without buckling the filament, the measurement was repeated with an interval of 30 seconds between applications. The pain threshold was determined as the tension of the filament (in grams) at which the mouse withdrew its paw in 3 out of 5 applications. The individual cages and all the set up were cleaned with a $30 \%$ ethanol solution between animals.

\section{Statistics}

Due to the limited number of animals $(n=2-4)$, no statistical analysis could be done. Data were expressed as mean \pm SEM. 


\subsection{Results}

\subsubsection{6-OHDA lesion effect on weight increment and water consumption. Evaluation of} ABA treatment

Since the start of ABA treatment at P28 (Fig. 6.1), weight and water intake were monitored once a week. The results are presented as the means \pm SEM ( $n=2-4$ per group).

In females, weight increment in sham group was from $15.70 \pm 0.49 \mathrm{~g}$ (week 1) to $18.63 \pm$ $0.23 \mathrm{~g}$ (week 4); in the 6-OHDA group from $13.90 \pm 0.10 \mathrm{~g}$ (week 1) to $21.95 \pm 1.85 \mathrm{~g}$ (week 4); and in 6-OHDA+ABA group from $15.12 \pm 0.22 \mathrm{~g}$ (week 1) to $18.47 \pm 0.17 \mathrm{~g}$ (week 4). This represents an increment of: $118.67 \pm 2.40 \%$ for sham, $158 \pm 14 \%$ for lesioned and $122.25 \pm 3.09$ $\%$ for lesioned + ABA (Fig. 6.2A)

In males, the sham group body weight increased from $19.70 \pm 0.78 \mathrm{~g}$ (week 1) to $25.23 \pm$ $0.27 \mathrm{~g}$ (week 4), lesioned mice from $20.77 \pm 0.14 \mathrm{~g}$ (week 1) to $23.50 \pm 0.49 \mathrm{~g}$ (week 4); and lesioned $+\mathrm{ABA}$ male exhibited the highest increment, from $15.53 \pm 0.34 \mathrm{~g}$ (week 1) to $24.70 \pm 0.51$ $\mathrm{g}$ (week 4). This represents an increment of $128.67 \pm 5.78 \%$ for sham; $113 \pm 2.08 \%$, for 6 -OHDA, and $159 \pm 6.66 \%$ for 6 -OHDA+ABA (Fig. 6.2B). These results suggest that lesion had opposite effects in males and females.

Weekly water consumption was registered per cage and it is represented by the mean $(\mathrm{mL})$ of the 4 weeks ( 1 cage per group). In females, 6-OHDA+ABA group average water intake was $111.25 \mathrm{~mL}$, followed by the sham female mice $(98.75 \mathrm{~mL})$ and 6-OHDA female mice $(76.25 \mathrm{~mL})$ (Fig. 6.2C). Males water intake was $117.5 \mathrm{~mL}$ for the 6-OHDA+ABA group, $97.5 \mathrm{~mL}$ for sham group and $93.75 \mathrm{~mL}$ for 6-OHDA group (Fig. 6.2D). We determined the ABA intake based on the monitoring of water consumption for the two 6-OHDA groups that received ABA: females consumption was $0.84 \mathrm{mg} /$ week/cage and males $0.87 \mathrm{mg} /$ week/cage (Fig. 6.2E). 
A

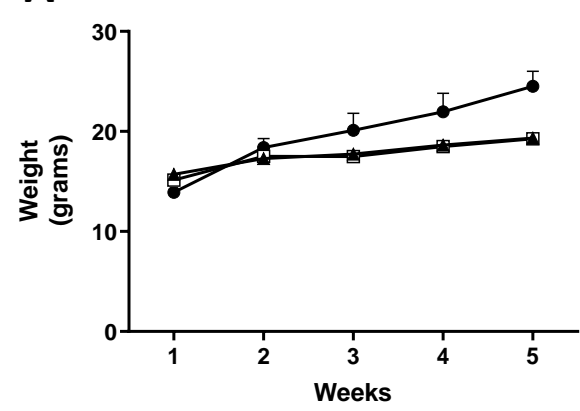

C

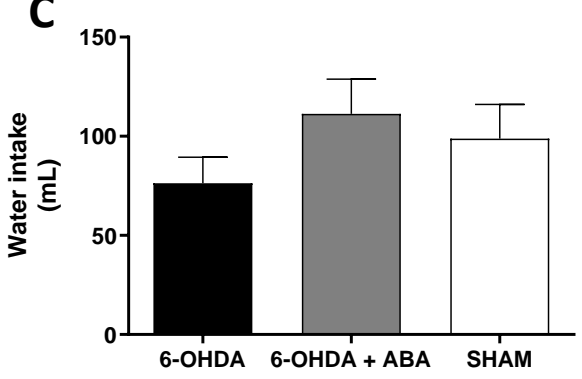

B

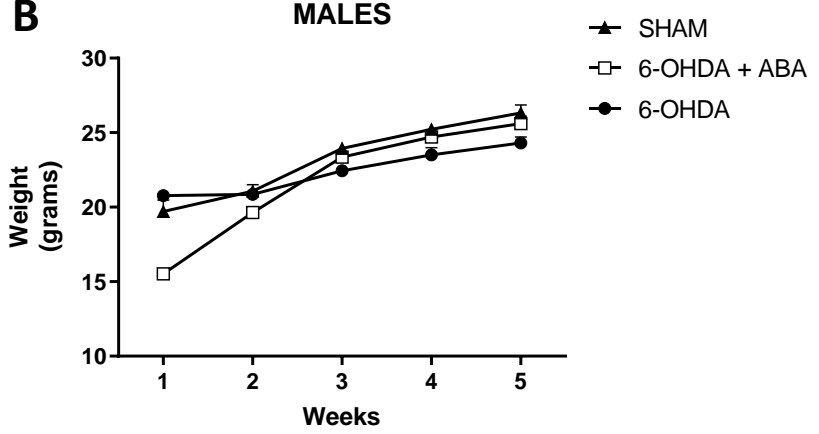

D

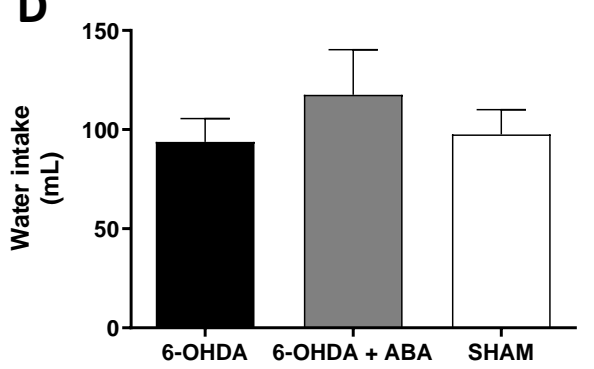

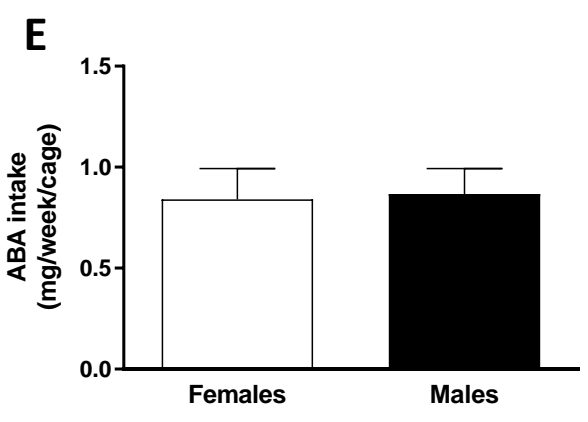

Fig. 6.2. Body weight (grams) and water intake $(\mathrm{mL})$ of 6-OHDA (black circles or black columns), 6-OHDA with ABA (6-OHDA+ABA, white squares, or grey columns) and Sham (black triangles or white columns) females (A, C) and males (B, D) along the 30 days of ABA treatment $(8 \mathrm{mg} / \mathrm{L}$ in the drinking water). ABA consumption of 6-OHDA-injected females and males was weekly measure per cage (E). $n=2-4$ per group. Values are mean \pm SEM.

\subsubsection{6-OHDA lesion effect on impulsivity}

Another distinctive trait of ADHD children is the impulsivity ${ }^{[409,410]}$. The CAR test is based on the tendency of rodents to avoid falling from high surfaces, so that animals with an impulsivity disorder will be more likely to jump out from the platform. The results are presented as the means \pm SEM ( $n=3-6$ per group). In females, the sham group jumped $(2.67 \pm 2.67$ number of falls $)$ and 6-OHDA jumped more ( $6.17 \pm 5.78$ number of falls) (Fig. 6.3A). However, the latency to the first fall was similar in both, lesioned $(46.92 \pm 9.16 \mathrm{~min})$ and sham $(43.33 \pm 16.67 \mathrm{~min})$ (Fig. 6.3C). Suggesting that lesion may have increased impulsivity in females. None of the sham male mice jumped out of the platform, whereas the 6-OHDA group jumped 4.50 \pm 4.11 times (Fig. 6.3B) with a latency of $47.92 \pm 9.56$ (Fig. 6.3D). Also in males, lesion seems to increase impulsivity. 
FEMALES

A
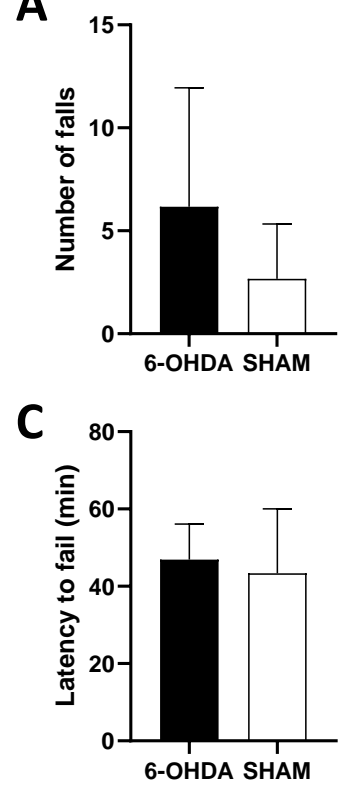

MALES

B
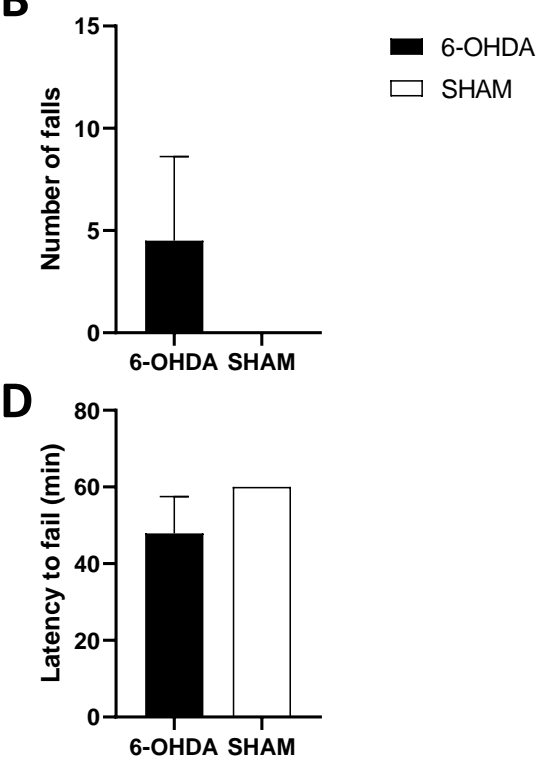

Fig. 6.3. Impulsivity. Cliff avoidance reaction (CAR) test data of 6-OHDA-injected mice (6OHDA, black columns) $(\mathrm{n}=6)$ and vehicle-injected mice (SHAM, white columns) $(\mathrm{n}=3)$ for females (A, C) and males (B, D). Number of falls (A, B) and latency to the first fall (min; C, D) were recorded at P26. Data are presented as mean \pm SEM.

\subsubsection{6-OHDA lesion effect on the spontaneous locomotor hyperactivity. Evaluation of ABA} treatment

One of the main and characteristic symptoms in ADHD is the spontaneous locomotor hyperactivity and it has been shown in mice ${ }^{[388]}$, rats ${ }^{[411]}$ and humans ${ }^{[412]}$. We used the open filed paradigm to evaluate spontaneous locomotor hyperactivity in our model. Velocity $(\mathrm{cm} / \mathrm{sec})$ and distance travelled $(\mathrm{cm})$ were recorded and are represented by the average of the two days of test. The results are presented as the means \pm SEM ( $n=2-4$ per group).

At P24-25, female's data for sham group were: $3.71 \pm 0.31 \mathrm{~cm} / \mathrm{s}$ (velocity) and $2216 \pm 181.6$ $\mathrm{cm}$ (distance); lesion decreased velocity (6-OHDA-VEH $3.26 \pm 0.01 \mathrm{~cm} / \mathrm{s}$; 6-OHDA+ABA $3.41 \pm$ $0.23 \mathrm{~cm} / \mathrm{s}$ ) and reduced distance (6-OHDA-VEH $1792 \pm 155.2 \mathrm{~cm} ; 6-\mathrm{OHDA}+\mathrm{ABA} 1989 \pm 137.3$ $\mathrm{cm})$. Thus, suggesting that the neonatal lesion decreased spontaneous locomotor activity in females. Four weeks after; sham females increased velocity $(4.26 \pm 0.51 \mathrm{~cm} / \mathrm{s})$ and distance $(2609$ $\pm 407.10 \mathrm{~cm}$ ) respect to P24-25. 6-OHDA-VEH females (velocity: $3.94 \pm 0.73 \mathrm{~cm} / \mathrm{s}$; distance: 2316 $\pm 428.9 \mathrm{~cm}$ ) also increased respect to P24-25. 6-OHDA+ABA group increased the most: velocity $(5.33 \pm 0.24 \mathrm{~cm} / \mathrm{s})$ and distance $(3040 \pm 119.5 \mathrm{~cm})$ (Fig. 6.4A, B). Thus, suggesting that ABA induced hyperactivity in the lesioned group.

In males, sham activity at P24-25 had lower velocity $(3.86 \pm 0.14 \mathrm{~cm} / \mathrm{s})$ and distance $(2307 \pm$ $80.26 \mathrm{~cm}$ ) than lesioned male mice velocity (6-OHDA-VEH $4.54 \pm 0.44 \mathrm{~cm} / \mathrm{s} ; 6-O H D A+A B A$ $4.28 \pm 0.63 \mathrm{~cm} / \mathrm{s}$ ) and distance (6-OHDA-VEH $2660 \pm 262.2 \mathrm{~cm} ; 6-\mathrm{OHDA}+\mathrm{ABA} 2552 \pm 374.6$ $\mathrm{cm})$. Indicating that neonatal lesion had increased spontaneous locomotor activity (as expected). After 4 weeks of treatment; sham males increased activity, velocity $(4.81 \pm 0.39 \mathrm{~cm} / \mathrm{s})$ and distance $(2780 \pm 249.7 \mathrm{~cm}$ ), but none of the lesioned groups: 6-OHDA-VEH (velocity: $4.17 \pm 0.37 \mathrm{~cm} / \mathrm{s}$; distance: $2446 \pm 218.9 \mathrm{~cm}$ ) and 6-OHDA+ABA group (velocity: $4.06 \pm 0.29 \mathrm{~cm} / \mathrm{s}$; distance: 2377 $\pm 179.30 \mathrm{~cm}$ ) (Fig. 6.4C, D). Suggesting that ABA had no effect in the lesioned group spontaneous locomotor activity, contrary to females. 

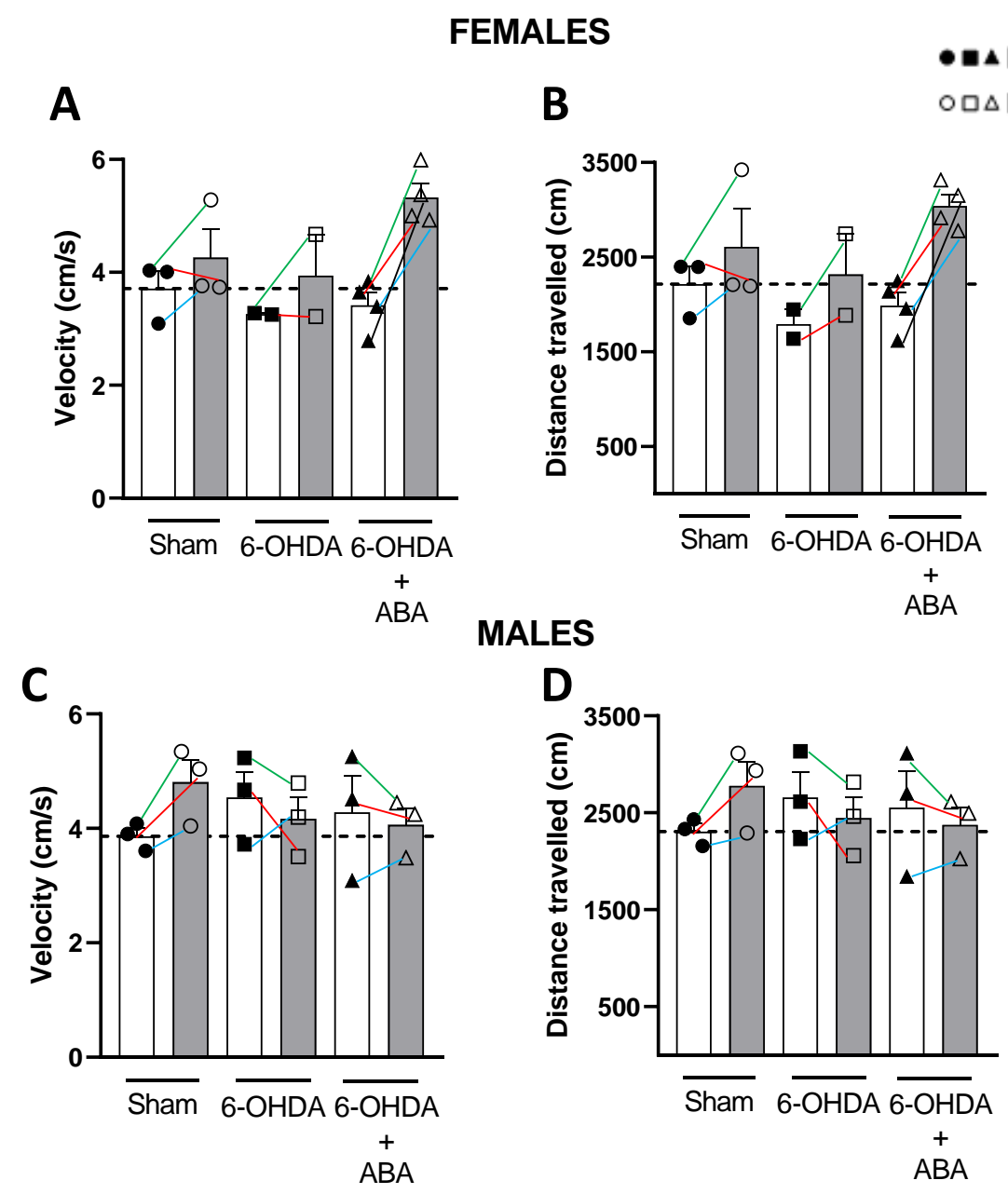

Fig. 6.4. Spontaneous locomotor hyperactivity. Open field data of 6-OHDA-injected mice (6OHDA, squares) $(\mathrm{n}=2-3)$, vehicle-injected mice (SHAM, circles) $(\mathrm{n}=3)$ and 6-OHDA animals that received ABA in the drinking water $(8 \mathrm{mg} / \mathrm{L})(6-\mathrm{OHDA}+\mathrm{ABA}$, triangles) $(\mathrm{n}=3-4)$ for females (A, B) and males (C, D) at the baseline (P24-P25; white columns with filled symbols) and after 1 month of treatment (ABA or VEH) (P56-P57; grey columns with empty symbols). Velocity $(\mathrm{cm} / \mathrm{sec})(\mathrm{A}, \mathrm{C})$ and distance travelled $(\mathrm{cm})(\mathrm{B}, \mathrm{D})$ were recorded. Values are the mean of both test days and data are presented as mean \pm SEM.

\subsubsection{6-OHDA lesion effect on pain hypersensitivity. Evaluation of ABA treatment}

Some clinical studies with children and adults with ADHD have reported an elevated pain perception in these patients ${ }^{[406,413,414]}$. To evaluate pain sensitivity, we performed two pain behaviour tests: plantar and Von Frey test. Plantar test quantifies the latency to paw withdrawal upon increasing thermal stimulus. The lower latency, the higher sensitivity to stimulus. Values are calculated by the average of 5 measures in both paws (left and right hind paws) and the results are presented as the mean \pm SEM ( $n=3-6$ per group). 6-OHDA-injection seems to lower pain threshold (as expected). Sham females stood $4.01 \pm 0.10 \mathrm{sec}$ without withdrew the paws and 6OHDA females $3.68 \pm 0.37 \mathrm{sec}$ (Fig. 6.5A). Meanwhile, sham males had a latency score of $3.77 \pm$ $0.93 \mathrm{sec}$ and 6-OHDA males had a score of $3.17 \pm 0.45 \mathrm{sec}$ (Fig. 6.5B). The effect of the lesion seems to be more noticeable in males. 

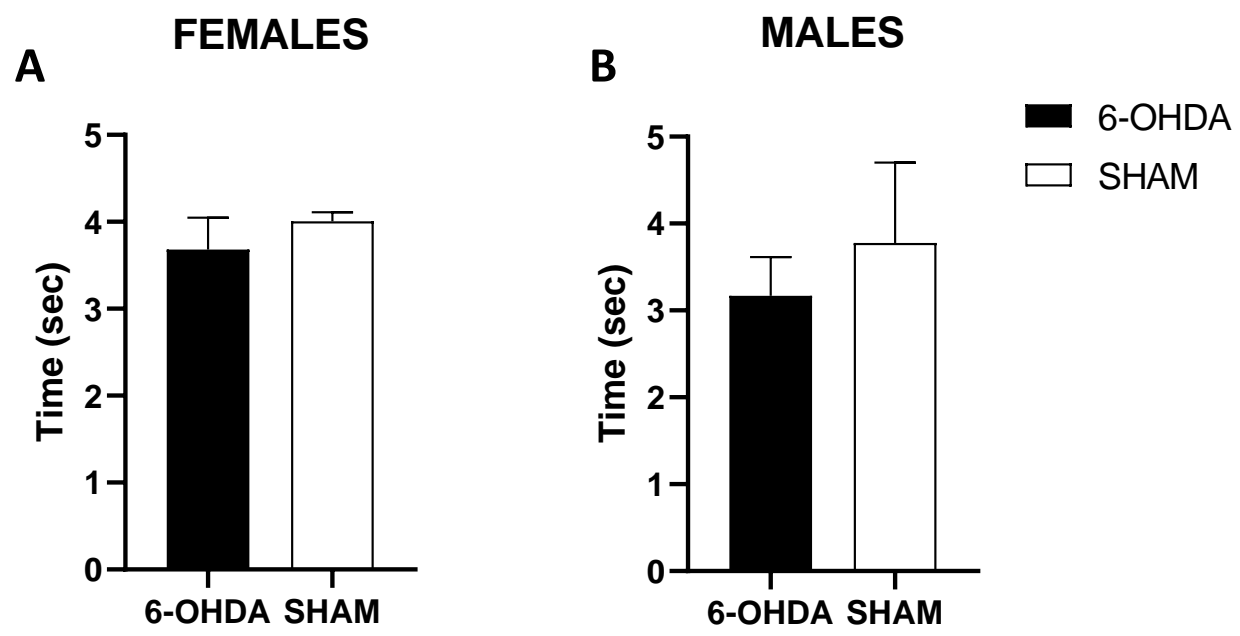

Fig. 6.5. Thermal stimulation (pain threshold). Plantar (Hargreaves apparatus) test data of 6OHDA-injected mice (6-OHDA, black columns) $(\mathrm{n}=6)$ and vehicle-injected mice (SHAM, white columns) $(\mathrm{n}=3)$ for females $(\mathrm{A})$ and males $(\mathrm{B})$. Latency $(\mathrm{sec})$ to the paw withdrawal under a thermal stimulus (IRed intensity of 50) was recorded at P27 (baseline). Values are the mean of 5 measurements and both paws (left hind paw and right hind paw). Data are presented as mean \pm SEM.

Unlike the plantar test, Von Frey test uses a mechanical stimulus to induce pain. The tension of the filament (in grams) at which the animal withdrew the paw was recorded and results are presented as the average of the two hind paws. Values are expressed as the means \pm SEM $(n=2-4$ per group). To evaluate the effect of $\mathrm{ABA}$ treatment animals were subjected to the Von Frey test at P27 (baseline) and P58 (post-treatment).

Sham females could not be correctly measured at P28, but at P58, showed a pain threshold of $1.23 \pm 0.23 \mathrm{~g}$. After a 4-week treatment of $\mathrm{VEH}$ or ABA, the 6-OHDA+ABA female group lower the threshold $(0.95 \pm 0.09 \mathrm{~g})$ compared to 6-OHDA-VEH $(1.35 \pm 0.35 \mathrm{~g})$, suggesting a potential increased of pain sensitization by ABA treatment (Fig. 6.6A).

In males, sham displayed a pain threshold of $2.07 \pm 0.98 \mathrm{~g}$ at P28 and after 4 weeks threshold was lower, $1.47 \pm 0.14 \mathrm{~g}$ (Fig. 6.6B). The 6-OHDA males showed a baseline pain threshold of $1.40 \pm 0.10 \mathrm{~g}$ (6-OHDA in Fig. 6.6) and $1.13 \pm 0.13 \mathrm{~g}$ (6-OHDA+ABA in Fig. 6.6) and, after 4 weeks, it was much lower (6-OHDA $0.87 \pm 0.07 \mathrm{~g})$, whereas 6-OHDA+ABA males were equal to P58 $(1.23 \pm 0.12 \mathrm{~g})$; suggesting that $\mathrm{ABA}$ may counteract lesion effect in pain. Opposite to what we observed in females.

This pilot study indicates that perhaps ABA treatment may have totally opposite effects in both sexes. The confirmation of these preliminary results is of huge relevance in the understanding of sex differences underlying disease symptoms, and to design effective treatments. 

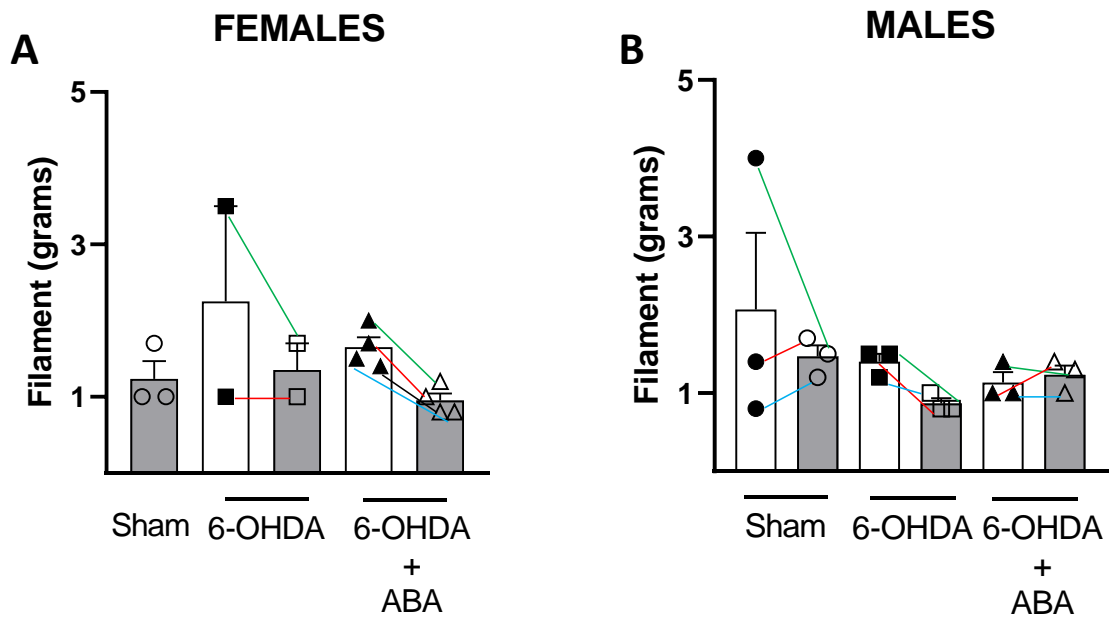

- - $\Delta$ Baseline

or $\triangle$ Post-treatment

Fig. 6.6. Mechanical stimulation (pain threshold). Von Frey test data of 6-OHDA-injected mice (6-OHDA, squares) $(n=2-3)$, vehicle-injected mice (SHAM, circles) $(n=3)$ and 6-OHDA animals that received $\mathrm{ABA}$ in the drinking water $(8 \mathrm{mg} / \mathrm{L})(6-\mathrm{OHDA}+\mathrm{ABA}$, triangles $)(\mathrm{n}=3-4)$ for females (A) and males (B) at the baseline (P28; white columns with filled symbols) and after 1 month of treatment (ABA or VEH) (P58; grey columns with empty symbols). The pain threshold based on calibrated filament tension (in grams) was recorded. Values are the mean of both paws (left hind paw and right hind paw). Data are presented as mean \pm SEM. 


\subsection{Discussion}

In this pilot study, we evaluated the effect of $\mathrm{ABA}$, a phytohormone with anti-inflammatory properties, in a 6-OHDA neonatal lesion model of ADHD in females and male mice. The aim is to ascertain if ABA can ameliorate the symptoms in psychiatric disease conditions, as we have shown for learning and memory tasks in animal models of inflammatory conditions.

6-OHDA lesion model is a validated Parkinson's disease (PD) model due to its specific neurotoxicity against dopaminergic neurons ${ }^{[415,416]}$. This DA depletion in neonatal mice has been demonstrated to induce ADHD-like behaviour ${ }^{[388]}$. To assess if we could counteract ADHD symptoms through reducing neuroinflammation, we treated animals with ABA in the drinking water $(8 \mathrm{mg} / \mathrm{L})$. All groups showed a similar water intake (as expected by our previous studies). Weight was also monitored. Males had a higher body weight gain compared with females, as expected for rodents. Interestingly, 6-OHDA-injected females showed bigger weight increment than sham, reaching male weight by two months. However, lesioned males had lower weight increment. The effect on females has never been reported before, thus pointing to a putative effect of sex in the association between dopaminergic system alterations, and body weight gain and obesity ${ }^{[417,418]}$. Some studies indicate that hormones such as estradiol promote oxidative stress and DA depletion in females Hyp, which is an area involved in feeding behaviour ${ }^{[419]}$. Thus, females could be more sensitive to DA reduction effects on weight. Males with lesion, on the other hand had lower weight than sham, perhaps due to increased activity.

Most interestingly, ABA administration seems to counteract the effect on body weight in both lesioned groups, that is decreasing increment in the females and increasing it in males.

To evaluate ADHD symptoms, we measured the performance of all mice in different paradigms: hyperactivity (open field paradigm), impulsivity (CAR test), and pain sensitivity (plantar and Von Frey tests).

In the open field paradigm, the more distance travelled, and the higher speed indicates higher spontaneous locomotor activity. We consider that sham animals with no treatment is the "normal behaviour" for this strain in these conditions. We have observed that lesion seemed to increase speed and distance in males, as reported ${ }^{[388,420]}$, however it seems to reduce activity in females. If we confirm this preliminary data, this finding had never been reported before and constitutes a considerable sex difference in the response to DA lesion.

In addition, both female and male controls increased activity after 4 weeks (no treatment), while ABA treatment does not seem to affect locomotor activity in lesioned males, it increases in females. This could suggest sex-dependent effects of ABA in lesioned subjects. Some studies have reported sex-differences in nutraceuticals' action (e.g. curcumin, also a PPAR $\gamma$ activator ${ }^{[421]}$ ), Perhaps due to different metabolism absorption ${ }^{[422]}$. Thus, we cannot rule out that possibility.

In the CAR test, impulsivity is measured by the jumps: the more jumps and lower latency to jump, the higher impulsivity. Control females had higher impulsivity compared to control males. Lesion had no effect in females, but increased impulsivity in males (as expected). To our knowledge this sex differences in impulsivity had never been reported before. The test was not performed after VEH or ABA treatment, so we cannot conclude the ABA effect on it.

In our pilot study, we have shown that the dopaminergic lesion model may affect differently males and females hyperactivity and impulsivity. Some studies indicates that there are sex differences in the striatum DA synthesis, so the impact of DA depletion can differ in males and females brain circuitry ${ }^{[423]}$. Of course, the confirmation of DA lesion must be done as well, to ascertain the reach of lesion and areas in both sexes.

Inflammation and pain are two closely linked fields. In this pilot study, plantar test results suggested that sham females have higher threshold than sham males (more tolerant to pain). Males inoculated with 6-OHDA had increased pain sensitization compared with sham controls (as 
expected), but this effect seemed smaller in females. Von Frey test was done at P27 and P58; we observed that ABA prevented the lesion effect in males (thus ameliorating pain sensitization) but not in females, where it had the opposite effect. If these data were to be confirmed, it would mean that ABA, perhaps increasing PPAR $\gamma$, could be associated to higher pain perception in females. Similarly, increased PPAR $\gamma$ has been found associated to pain in women suffering peritoneal endometriotic lesions ${ }^{[424]}$. Thus, we could speculate that the excessive PPAR $\gamma$-agonist action might exert negative effects in females and therefore $\mathrm{ABA}$ treatment for pain must be carefully evaluated.

Up until now, there are no reports about C57BL/6 mice strain in neonatal 6-OHDA-lesioned model. Another major novelty of this pilot study is the comparative analysis of this model in female and male mice. Moreover, these preliminary results point to sex differences in ADHD symptomatology, pain perception and, importantly, in therapeutic strategy. 


\section{7}

GENERAL DISCUSSION

AND

FUTURE DIRECTIONS 
Our findings confirm the relevance of insulin resistance and inflammation inducing memory alterations, and how ABA, a molecule with insulin-sensitizing and anti-inflammatory properties is a potential treatment. Moreover, we dissect insulin resistance from inflammation, and proved that reduced IRS1 expression impairs spatial memory, in the absence of inflammation. In that study, we started to point to important sex-differences in susceptibility to IRS1 deficits. Finally, in our pilot study, we further confirm the absolute necessity to carry out comparative studies in both females and males, with particular emphasis on therapeutic interventions.

\subsection{The potential therapeutic value of $A B A$, in male rodents}

In this thesis, we confirm that $\mathrm{ABA}$ administered in the drinking water improved peripheral glucose tolerance and cognitive alterations in a model of inflammation induced by HFD. T-maze and novel object recognition paradigms have been validated as reliable spatial and recognition memory tasks respectively ${ }^{[210,234]}$. We would need to analyse whether the progression of inflammation affects sequentially different types of memory functions, as happens in AD patients with an early impaired episodic memory ${ }^{[425]}$, then ABA rescue effect is limited in time depending on disease progression.

Impaired IRS1 or IRS2 response can lead to insulin resistance. In our work, we demonstrated for the first time that HFD differently alters hippocampal IRS1 and IRS2 mRNA expression, IRS2 expression is reduced whereas IRS1 expression is increased, which could indicate different roles in neuroinflammation and insulin resistance. We show that ABA counteracts this effect in addition to reduce HFD-induced inflammatory markers (reactive microglia and pro-inflammatory cytokines), improved hippocampal neurogenesis, and restored APP (increased by HFD) and BDNF (reduced by HFD) expression. We concluded that ABA treatment may have an effect on the mRNA promoter activity of these genes, likely via PPAR $\gamma$ activation ${ }^{[147]}$, in accordance with previous data, showing that PPAR $\gamma$ agonists administration in APP/PS1 and diabetic transgenic mice restored IRS $1^{[299]}$ and IRS2 ${ }^{[144]}$ expression, respectively.

Other PPAR $\gamma$ agonist (synthetic insulin sensitizer molecules, e.g. TZDs) have been reported to reduce pro-inflammatory cytokines in patients with rheumatoid arthritis ${ }^{[426]}$. However, these drugs have undesirable side effects ${ }^{[195-197]}$. ABA shares molecular mechanisms with TZDs, but we postulate that $\mathrm{ABA}$ treatment could be a safer natural alternative to synthetic insulin sensitizer molecules.

Finally, we consider that the effects on hippocampal BDNF, IRS1, IRS2, APP, cytokines expression and neurogenesis are critical for the cognitive beneficial properties of $A B A$, however, whether is a peripheral benefit effect on brain or a direct effect on brain neuronal and astroglia cells is currently unknown. In addition, we would like to discern if ABA action as insulin sensitizer downregulates inflammation or is the anti-inflammatory properties that lower inflammation.

Moreover, the effect of $\mathrm{ABA}$ in control animals, sometimes worsen the results, is very interesting and points to optimal levels not lower, but also not higher, if we consider that ABA can be synthesized endogenously in healthy individuals, then we can infer that we are "adding" ABA perhaps too higher than optimal levels. The optimal range need to be determined.

Last, but not least, the effect of ABA on female rats, both controls and fed with a HFD need to be elucidated. 


\subsection{Insulin resistance impairs spatial and recognition memory in females, only spatial memories in males}

Insulin resistance animal models and clinical diseases are usually accompanied by increased inflammation, thus the relative contribution to cognitive impairment is uncertain. In this thesis, we have elucidated the effect of reducing IRS1 signalling on cognitive function, in the absence of inflammation, by using an adeno-associated virus (AAV) expressing shRNA targeting IRS1 mRNA to knock down its expression specifically. Whether this IRS1 reduction resulted in "insulin resistance" was only inferred by the western blot analysis in cultured cells treated with insulin, but it would need to be determined in vivo. Moreover, whether this IRS1 reduction increases inflammatory markers or worsen a possible inflammatory effect of stereotaxic injection, also remains to be elucidated.

There is an association of hippocampal alterations with clinical anxiety and depression ${ }^{[427,428]}$. In rodents, this function is associated with ventral HPC ${ }^{[429]}$, area not affected by our AAVinjection (data not shown), which may explain why we did not find these symptoms in our model. We observed that AAV DJ8 virus affects mostly GABAergic hippocampal neurons which inhibit locally and to other areas, such as the MS. Since this area is particularly involved in memory function, we consider that specific intervention on this circuit may contribute to the absence of anxiety or depressive symptoms. AAV inoculations affecting ventral HPC would be required to answer that question. Moreover, the AAV shIRS1 will help us to analyse the effects of IRS1 knocking down in other brain areas. Furthermore, the comparative study of IRS2 in same circuit remains to be elucidated.

We have revised that insulin signalling promotes neuronal survival and energy homeostasis ${ }^{[31,110-112,430,431]}$, and it is required for neuroplasticity and spatial memory ${ }^{[32]}$. Insulin is also required for glutamate receptors ${ }^{[25,27]}$ and PSD-95 expression at the surface membrane ${ }^{[29]}$, and optimal synaptic plasticity ${ }^{[432]}$. Our data indicates that the roles reported for IR in synaptic plasticity could be mediated by IRS1, since we have found that IRS1 downregulation reduces synaptic plasticity by indirect measures of presynaptic marker (synaptophysin). The molecular mechanism underlying synaptophysin alteration by IRS1 silencing is unknown. Since IRS1 is required for PI3K-Akt pathway-induced GLUT4 translocation to plasma membrane ${ }^{[433]}$, we postulate that this pathway is involved in preventing the extra glucose needed for activity, perhaps due to the translocation of other synaptic proteins. These mechanisms remain to be elucidated.

Furthermore, IRS1 downregulation could also induce postsynaptic activity alterations, thus it should be analysed in further studies for instance through electrophysiological techniques that would help to better understand the effects on both pre and postsynaptic currents.

In our work, we used AAV DJ8 serotype, which has shown a preference for GABAergic neurons. Thus, the effect of IRS1 silencing in glutamatergic neurons remains to be elucidated. This can be achieved by, for example, changing AAV tropism (different serotypes) to improve infection in different neuronal types.

Comparative studies of females and males are still minority in preclinical field. Still, different meta-analyses unveiled that males performs better spatial navigation and working memory tasks than females, in clinical ${ }^{[434]}$ (pointing to social as well as biological differences), but also in preclinical studies ${ }^{[435]}$, (suggesting just biological differences). In patients suffering dementia, women displayed worse memory deficits ${ }^{[436]}$ and faster temporal lobe atrophy ${ }^{[437]}$ than men, and the prevalence of $\mathrm{AD}$ is higher in women ${ }^{[346]}$. Furthermore, insulin resistance can be induced by hormonal alterations (e.g. polycystic ovary syndrome) in absence of obesity condition ${ }^{[438]}$. Also estrogen levels are associated with less-effective transport of insulin into the CNS ${ }^{[439]}$.

In this thesis, we carry out an approach to evaluate sex differences in memory tasks. We found that IRS1 reduction affected spatial memory in both females and males, whereas recognition memory was only affected in females. Our study was conducted with non-ovariectomized rats, thus the insulin resistance susceptibility difference may be due to hormonal modulated events. These 
results confirm that insulin resistance may have a higher impact in females. Whether this is due estrogen synergy with IRS1 deficits (e.g. mitochondria function ${ }^{[440]}$, AMPA receptors stoichiometry ${ }^{[441]}$, spine density of pyramidal neurons ${ }^{[442]}$, LTP and or LTD $^{[443]}$ ) remains unknown and it is an objective in future research. 


\subsection{ADHD model: potential sex-differences}

Even with different aetiology than neurological diseases, psychiatric disorders have also been associated to neuroinflammation, e.g. major depressive disorder (MDD) ${ }^{[444]}$, bipolar disorder ${ }^{[445]}$. In this thesis, we show the preliminary data of a pilot study of ABA effects on a model of ADHD in both males and females. To our knowledge, it is the first comparative study attempting to associate inflammation and ADHD in preclinical model, and pain sensitization in both female and males.

Hyperexcitability derived of neuroinflammation in spinal cord and brain causes pain central sensitization ${ }^{[46]}$. Specifically, pro-inflammatory cytokines promote hypersensitivity of nociceptors (pain receptors) ${ }^{[447]}$. The continuous releasing of those inflammatory cytokines leads to chronic widespread pain that is extended to mostly all the body ${ }^{[447]}$ and can result in chronic pain disease [448]. Our data suggests that this model may be a useful tool to understand ADHD and pain relationship.

Early exposure to inflammatory processes may be a possible risk factor to develop ADHD ${ }^{[390,449-451]}$. Also, growing evidence suggests that there is a link between pain and ADHD ${ }^{[452-455]}$, but it is still a relatively unexplored field, especially in preclinical studies. Thus, we hypothesized that anti-inflammatory treatments could be at the crossroad of ADHD traits and pain symptoms, then ameliorating the disease. However, our data supports that same treatment may not be used equally in both females and males.

In other preclinical studies, estradiol can promote the inflammatory response by increasing GFAP and IL-1 $\beta$ expression; oxidative stress and DA depletion in Hyp ${ }^{[419]}$. Also in humans, sex differences have been found, supporting that ADHD prevalence is higher in males than females ${ }^{[456,457]}$ also with different symptomatology: whereas impulsivity is prevalent in males, inattention trait seems predominant in women ${ }^{[458]}$. However, in humans there are not only biological differences, but also social and familiar influences.

Our data support sex-differences in basal behavior, lesion-induced behavior and even treatment effects. Thus, tentatively we suggest that: i) females are more active and impulsive than male in the basal state; ii) neonatal DA depletion may not promote hyperactivity/impulsivity traits in females, as it does in males, iii) ABA treatment seems to improve ADHD behavioural traits in males but not females, where it seems to even worsen them, iv) ABA appears to counteract the body weight gain observed in females, perhaps by increasing hyperactivity. Therefore, further studies should be carried out to understand the underlying mechanisms. This would be necessary for development of suitable anti-inflammatory treatments against ADHD symptoms.

If these results are confirmed, they will greatly contribute to further understand sex differences in the neuroinflammatory etiology of ADHD and whether ABA could be a potential treatment to ameliorate classic traits and pain hypersensitization. Even though sex differences in inflammatory response are known (e.g. differences in circulating monocytes ${ }^{[459]}$ and microglia $\left.{ }^{[460,461]}\right)$, the mechanisms explaining potential opposite effects of ABA remains to be elucidated.

These data strongly endorse to always carry out comparative studies with females and males, since treatments and symptomatology may have very different outcomes under certain conditions. 
8

\section{CONCLUSIONS}


$\mathrm{ABA}$ treatment in a male rat model of high fat diet-induced neuroinflammation:

1) restores spatial and recognition memory function, concomitant with increasing peripheral insulin sensitivity.

2) crosses the blood brain barrier.

3) reduces neuroinflammation in hypothalamus and hippocampus.

4) improves neurogenesis in hippocampus.

5) restores hippocampal IRS1 and IRS2 expression levels that are differentially affected in this model.

6) restores hippocampal BDNF and APP expression levels in hippocampus, affected by this model.

7) in sham animals may worsen some parameters, pointing to a window of beneficial action.

AAV-CMV-shIRS1-EGFP inoculation in dorsal hippocampus, compared to AAV-CMVEGFP control:

8) both labelled preferentially hilar GABAergic neurons.

9) reduces IRS1 expression in hippocampal hilar neurons.

10) in females is sufficient to cause spatial and recognition memory deficits in the absence of neuroinflammation.

11) in males causes only spatial memory alteration in the absence of neuroinflammation.

12) in females modulates the synaptic plasticity in GABAergic hippocampal neurons projecting to medial septum.

13) does not alter anxiety symptomatology.

ABA treatment in a 6-OHDA neonatal lesion model of ADHD (Pilot study):

14) highlights the potential differences due to sex-differences in drug effects and/or brain lesions models, and the need to evaluate these in both sexes. Points to a revision of disease preclinical models and pharmacological treatments in comparative females $v s$ males studies. 


\section{9}

BIBLIOGRAPHY 
1. Havrankova J, Roth J, Brownstein M. Insulin receptors are widely distributed in the central nervous system of the rat. Nature. 1978;272(5656):827-9. DOI: 10.1038/272827a0.

2. Mayer JP, Zhang F, DiMarchi RD. Insulin structure and function. Vol. 88, Biopolymers - Peptide Science Section. Biopolymers; 2007. p. 687-713. DOI: 10.1002/bip.20734.

3. Csajbók ÉA, Tamás G. Cerebral cortex: a target and source of insulin?. Vol. 59, Diabetologia. Springer Verlag; 2016. p. 1609-15. DOI: 10.1007/s00125-016-3996-2.

4. Gray SM, Aylor KW, Barrett EJ. Unravelling the regulation of insulin transport across the brain endothelial cell. Diabetologia. 2017 Aug 1;60(8):1512-21. DOI: 10.1007/s00125-017-4285-4.

5. Banks WA, Jaspan JB, Kastin AJ. Effect of diabetes mellitus on the permeability of the blood-brain barrier to insulin. Peptides. 1997;18(10):1577-84. DOI: 10.1016/S0196-9781(97)00238-6.

6. Banks WA, Farr SA, Morley JE. Permeability of the blood-brain barrier to albumin and insulin in the young and aged SAMP8 mouse. Journals Gerontol - Ser A Biol Sci Med Sci. 2000;55(12):B601-6. DOI: 10.1093/gerona/55.12.B601.

7. Banks WA, Kastin AJ, Pan W. Uptake and degradation of blood-borne insulin by the olfactory bulb. Peptides. 1999;20(3):373-8. DOI: 10.1016/S0196-9781(99)00045-5.

8. Baskin DG, Porte D, Guest K, Dorsa DM. Regional concentrations of insulin in the rat brain. Endocrinology. 1983;112(3):898-903. DOI: 10.1210/endo-112-3-898.

9. Banks WA, Kastin AJ. Differential permeability of the blood-brain barrier to two pancreatic peptides: Insulin and amylin. Peptides. 1998;19(5):883-9. DOI: 10.1016/S0196-9781(98)00018-7.

10. Niswender KD. Basal insulin: Physiology, pharmacology, and clinical implications. Postgrad Med. 2011 Jul;123(4):17-26. DOI: 10.3810/pgm.2011.07.2300.

11. Garvey WT, Maianu L, Huecksteadt TP, Birnbaum MJ, Molina JM, Ciaraldi TP. Pretranslational suppression of a glucose transporter protein causes insulin resistance in adipocytes from patients with non-insulin-dependent diabetes mellitus and obesity. J Clin Invest. 1991;87(3):1072-81. DOI: 10.1172/jci115068.

12. Leloup C, Arluison M, Kassis N, Lepetit N, Cartier N, Ferré P, Pénicaud L. Discrete brain areas express the insulin-responsive glucose transporter GLUT4. Mol Brain Res. 1996;38(1):45-53. DOI: 10.1016/0169328X(95)00306-D.

13. Grillo CA, Piroli GG, Hendry RM, Reagan LP. Insulin-stimulated translocation of GLUT4 to the plasma membrane in rat hippocampus is PI3-kinase dependent. Brain Res. 2009 Oct 16;1296:35-45. DOI: 10.1016/j.brainres.2009.08.005.

14. McEwen BS, Reagan LP. Glucose transporter expression in the central nervous system: Relationship to synaptic function. Eur J Pharmacol. 2004 Apr 19;490(1-3):13-24. DOI: 10.1016/j.ejphar.2004.02.041.

15. Apelt J, Mehlhorn G, Schliebs R. Insulin-sensitive GLUT4 glucose transporters are colocalized with GLUT3expressing cells and demonstrate a chemically distinct neuron-specific localization in rat brain. J Neurosci Res. 1999 Sep 1;57(5):693-705. DOI: 10.1002/(SICI)1097-4547(19990901)57:5<693::AID-JNR11>3.0.CO;2-X.

16. Reagan LP, Gorovits N, Hoskin EK, Alves SE, Katz EB, Grillo CA, Piroli GG, McEwen BS, Charron MJ. Localization and regulation of GLUTx1 glucose transporter in the hippocampus of streptozotocin diabetic rats. Proc Natl Acad Sci U S A. 2001 Feb 27;98(5):2820-5. DOI: 10.1073/pnas.051629798.

17. Piroli GG, Grillo CA, Hoskin EK, Znamensky V, Katz EB, Milner TA, McEwen BS, Charron MJ, Reagan LP. Peripheral glucose administration stimulates the translocation of GLUT8 glucose transporter to the endoplasmic reticulum in the rat hippocampus. J Comp Neurol. 2002 Oct 14;452(2):103-14. DOI: 10.1002/cne.10368.

18. Schmidt S, Joost HG, Schürmann A. GLUT8, the enigmatic intracellular hexose transporter. Vol. 296, American Journal of Physiology - Endocrinology and Metabolism. Am J Physiol Endocrinol Metab; 2009. DOI: 10.1152/ajpendo.91019.2008.

19. Bruning JC, Gautam D, Burks DJ, Gillette J, Schubert M, Orban PC, Klein R, Krone W, Muller-Wieland D, Kahn CR. Role of brain insulin receptor in control of body weight and reproduction. Science (80- ). 2000 Sep 22;289(5487):2122-5. DOI: 10.1126/science.289.5487.2122.

20. Könner AC, Janoschek R, Plum L, Jordan SD, Rother E, Ma X, Xu C, Enriori P, Hampel B, Barsh GS, Kahn CR, Cowley MA, Ashcroft FM, Brüning JC. Insulin Action in AgRP-Expressing Neurons Is Required for Suppression of Hepatic Glucose Production. Cell Metab. 2007 Jun 6;5(6):438-49. DOI: 
10.1016/j.cmet.2007.05.004.

21. Cukierman T, Gerstein HC, Williamson JD. Cognitive decline and dementia in diabetes - Systematic overview of prospective observational studies. Vol. 48, Diabetologia. Diabetologia; 2005. p. 2460-9. DOI: $10.1007 / \mathrm{s} 00125-005-0023-4$.

22. Biessels GJ, Deary IJ, Ryan CM. Cognition and diabetes: a lifespan perspective. Vol. 7, The Lancet Neurology. Lancet Publishing Group; 2008. p. 184-90. DOI: 10.1016/S1474-4422(08)70021-8.

23. Den Heijer T, Vermeer SE, Van Dijk EJ, Prins ND, Koudstaal PJ, Hofman A, Breteler MMB. Type 2 diabetes and atrophy of medial temporal lobe structures on brain MRI. Diabetologia. 2003 Dec;46(12):1604-10. DOI: $10.1007 / \mathrm{s} 00125-003-1235-0$.

24. Musen G, Jacobson AM, Bolo NR, Simonson DC, Shenton ME, McCartney RL, Flores VL, Hoogenboom WS Resting-state brain functional connectivity is altered in type 2 diabetes. Diabetes. 2012 Sep;61(9):2375-9. DOI: $10.2337 / \mathrm{db} 11-1669$.

25. Shonesy BC, Thiruchelvam K, Parameshwaran K, Rahman EA, Karuppagounder SS, Huggins KW, Pinkert CA, Amin R, Dhanasekaran M, Suppiramaniam V. Central insulin resistance and synaptic dysfunction in intracerebroventricular-streptozotocin injected rodents. Neurobiol Aging. 2012 Feb;33(2):430.e5-18. DOI: 10.1016/j.neurobiolaging.2010.12.002.

26. Zhao F, Siu JJ, Huang W, Askwith C, Cao L. Insulin Modulates Excitatory Synaptic Transmission and Synaptic Plasticity in the Mouse Hippocampus. Neuroscience. 2019 Jul 15;411:237-54. DOI: 10.1016/j.neuroscience.2019.05.033.

27. Skeberdis VA, Lan J, Zheng X, Zukin RS, Bennett M V. Insulin promotes rapid delivery of N-methyl-Daspartate receptors to the cell surface by exocytosis. Proc Natl Acad Sci U S A. 2001 Mar 13;98(6):3561-6. DOI: 10.1073/pnas.051634698.

28. Christie JM, Wenthold RJ, Monaghan DT. Insulin causes a transient tyrosine phosphorylation of NR2A and NR2B NMDA receptor subunits in rat hippocampus. J Neurochem. 1999;72(4):1523-8. DOI: 10.1046/j.14714159.1999.721523.x.

29. Lee CC, Huang CC, Wu MY, Hsu K Sen. Insulin stimulates postsynaptic density-95 protein translation via the phosphoinositide 3-kinase-Akt-mammalian target of rapamycin signaling pathway. J Biol Chem. 2005 May 6;280(18):18543-50. DOI: 10.1074/jbc.M414112200.

30. Huang CC, Lee CC, Hsu K Sen. An investigation into signal transduction mechanisms involved in insulininduced long-term depression in the CA1 region of the hippocampus. J Neurochem. 2004;89(1):217-31. DOI: 10.1111/j.1471-4159.2003.02307.x.

31. Wan Q, Xiong ZG, Man HY, Ackerley CA, Braunton J, Lu WY, Becker LE, MacDonald JF, Wang YT. Recruitment of functional GABA(A) receptors to postsynaptic domains by insulin. Nature. 1997;388(6643):686-90. DOI: 10.1038/41792.

32. Grillo CA, Piroli GG, Lawrence RC, Wrighten SA, Green AJ, Wilson SP, Sakai RR, Kelly SJ, Wilson MA, Mott DD, Reagan LP. Hippocampal Insulin Resistance Impairs Spatial Learning and Synaptic Plasticity. Diabetes. 2015 Nov;64(11):3927-36. DOI: 10.2337/db15-0596.

33. Adzovic L, Lynn AE, D'Angelo HM, Crockett AM, Kaercher RM, Royer SE, Hopp SC, Wenk GL. Insulin improves memory and reduces chronic neuroinflammation in the hippocampus of young but not aged brains. $J$ Neuroinflammation. 2015 Apr 2;12(1). DOI: 10.1186/s12974-015-0282-z.

34. Moosavi M, Naghdi N, Maghsoudi N, Zahedi Asl S. Insulin protects against stress-induced impairments in water maze performance. Behav Brain Res. 2007 Jan 25;176(2):230-6. DOI: 10.1016/j.bbr.2006.10.011.

35. Craft S, Baker LD, Montine TJ, Minoshima S, Watson GS, Claxton A, Arbuckle M, Callaghan M, Tsai E, Plymate SR, Green PS, Leverenz J, Cross D, Gerton B. Intranasal insulin therapy for Alzheimer disease and amnestic mild cognitive impairment: A pilot clinical trial. Arch Neurol. 2012 Jan;69(1):29-38. DOI: 10.1001/archneurol.2011.233.

36. Kleinridders A, Pothos EN. Impact of Brain Insulin Signaling on Dopamine Function, Food Intake, Reward, and Emotional Behavior. Vol. 8, Current Nutrition Reports. Current Science Inc.; 2019. p. 83-91. DOI: 10.1007/s13668-019-0276-z.

37. Gupta D, Kurhe Y, Radhakrishnan M. Antidepressant effects of insulin in streptozotocin induced diabetic mice: Modulation of brain serotonin system. Physiol Behav. 2014 Apr 22;129:73-8. DOI: 10.1016/j.physbeh.2014.02.036. 
38. Radojičić R, Cvijić G, Djordjević J, Djurašević S, Davidović V. Chronic effect of insulin on monoamine oxidase and antioxidant enzyme activities in the rat brainstem. Comp Biochem Physiol - C Pharmacol Toxicol Endocrinol. 1997;117(2):187-92. DOI: 10.1016/S0742-8413(97)89893-3.

39. Figlewicz DP, Szot P, Chavez M, Woods SC, Veith RC. Intraventricular insulin increases dopamine transporter mRNA in rat VTA/substantia nigra. Brain Res. 1994 May 2;644(2):331-4.

40. Sharma AN, Elased KM, Lucot JB. Rosiglitazone treatment reversed depression- but not psychosis-like behavior of db/db diabetic mice. J Psychopharmacol. 2012 May;26(5):724-32. DOI: 10.1177/0269881111434620.

41. Heni M, Hennige AM, Peter A, Siegel-Axel D, Ordelheide AM, Krebs N, Machicao F, Fritsche A, Häring HU, Staiger H. Insulin promotes Glycogen storage and cell proliferation in primary human Astrocytes. PLoS One. 2011;6(6). DOI: 10.1371/journal.pone.0021594.

42. Ji YF, Xu SM, Zhu J, Wang XX, Shen Y. Insulin increases glutamate transporter GLT1 in cultured astrocytes. Biochem Biophys Res Commun. 2011 Feb 25;405(4):691-6. DOI: 10.1016/j.bbrc.2011.01.105.

43. Haas CB, de Carvalho AK, Muller AP, Eggen BJL, Portela LV. Insulin activates microglia and increases COX2/IL-1 $\beta$ expression in young but not in aged hippocampus. Brain Res. 2020 Aug 15;1741. DOI: 10.1016/j.brainres.2020.146884.

44. Haeusler RA, McGraw TE, Accili D. Metabolic Signalling: Biochemical and cellular properties of insulin receptor signalling. Vol. 19, Nature Reviews Molecular Cell Biology. Nature Publishing Group; 2018. p. 31-44. DOI: $10.1038 / \mathrm{nrm} .2017 .89$.

45. Tatulian SA. Structural Dynamics of Insulin Receptor and Transmembrane Signaling. Biochemistry. 2015 Sep 15;54(36):5523-32. DOI: 10.1021/acs.biochem.5b00805.

46. Hançer NJ, Qiu W, Cherella C, Li Y, Copps KD, White MF. Insulin and metabolic stress stimulate multisite serine/threonine phosphorylation of insulin receptor substrate 1 and inhibit tyrosine phosphorylation. $J$ Biol Chem. 2014;289(18):12467-84. DOI: 10.1074/jbc.M114.554162.

47. Pederson TM, Kramer DL, Rondinone CM. Serine/threonine phosphorylation of IRS-1 triggers its degradation: possible regulation by tyrosine phosphorylation. Diabetes. 2001 Jan;50(1):24-31.

48. Aguirre V, Werner ED, Giraud J, Lee YH, Shoelson SE, White MF. Phosphorylation of Ser307 in insulin receptor substrate-1 blocks interactions with the insulin receptor and inhibits insulin action. J Biol Chem. 2002 Jan;277(2):1531-7. DOI: 10.1074/jbc.M101521200.

49. Giovannone B, Scaldaferri ML, Federici M, Porzio O, Lauro D, Fusco A, Sbraccia P, Borboni P, Lauro R, Sesti G. Insulin receptor substrate (IRS) transduction system: Distinct and overlapping signaling potential [Internet]. Vol. 16, Diabetes/Metabolism Research and Reviews. Diabetes Metab Res Rev; 2000 [cited 2020 Dec 31]. p. 434-41. DOI: 10.1002/1520-7560(2000)9999:9999<::AID-DMRR159>3.0.CO;2-8.

50. Sesti G, Federici M, Hribal ML, Lauro D, Sbraccia P, Lauro R. Defects of the insulin receptor substrate (IRS) system in human metabolic disorders. FASEB J. 2001 Oct;15(12):2099-111. DOI: 10.1096/fj.01-0009rev.

51. Björnholm M, He AR, Attersand A, Lake S, Liu SCH, Lienhard GE, Taylor S, Arner P, Zierath JR. Absence of functional insulin receptor substrate-3 (IRS-3) gene in humans. Diabetologia. 2002;45(12):1697-702. DOI: 10.1007/s00125-002-0945-z.

52. Zhou L, Chen H, Xu P, Cong LN, Sciacchitano S, Li Y, Graham D, Jacobs AR, Taylor SI, Quon MJ. Action of insulin receptor substrate-3 (IRS-3) and IRS-4 to stimulate translocation of GLUT4 in rat adipose cells. Mol Endocrinol. 1999;13(3):505-14. DOI: 10.1210/mend.13.3.0242.

53. Liu SCH, Wang Q, Lienhard GE, Keller SR. Insulin receptor substrate 3 is not essential for growth or glucose homeostasis. J Biol Chem. 1999 Jun 18;274(25):18093-9. DOI: 10.1074/jbc.274.25.18093.

54. Fantin VR, Wang Q, Lienhard GE, Keller SR. Mice lacking insulin receptor substrate 4 exhibit mild defects in growth, reproduction, and glucose homeostasis. Am J Physiol - Endocrinol Metab. 2000;278(1 41-1). DOI: 10.1152/ajpendo.2000.278.1.e127.

55. Tanaka S, Ito T, Wands JR. Neoplastic transformation induced by insulin receptor substrate-1 overexpression requires an interaction with both Grb2 and Syp signaling molecules. J Biol Chem. 1996;271(24):14610-6. DOI: 10.1074/jbc.271.24.14610.

56. Ito T, Sasaki Y, Wands JR. Overexpression of human insulin receptor substrate 1 induces cellular transformation with activation of mitogen-activated protein kinases. Mol Cell Biol. 1996 Mar;16(3):943-51. DOI: 10.1128/mcb.16.3.943.

57. Jackson JG, White MF, Yee D. Insulin receptor substrate-1 is the predominant signaling molecule activated by 
insulin-like growth factor-I, insulin, and interleukin-4 in estrogen receptor-positive human breast cancer cells. $J$ Biol Chem. 1998 Apr 17;273(16):9994-10003. DOI: 10.1074/jbc.273.16.9994.

58. HAYASHI T, KUBOTA N, KUBOTA T, KADOWAKI T. Lack of Brain Insulin Receptor Substrate-1 (IRS1) Causes Growth Retardation via Growth Hormone Releasing Hormone. Diabetes. 2018 May 1;67(Supplement 1):1795-P. DOI: 10.2337/db18-1795-p.

59. Tamemoto H, Kadowaki T, Tobe K, Yagi T, Sakura H, Hayakawa T, Terauchi Y, Ueki K, Kaburagi Y, Satoh S, Sekihara H, Yoshioka S, Horikoshi H, Furuta Y, Ikawa Y, Kasuga M, Yazaki Y, Aizawa S. Insulin resistance and growth retardation in mice lacking insulin receptor substrate-1. Nature. 1994;372(6502):182-6. DOI: $10.1038 / 372182 \mathrm{a} 0$.

60. Sawka-Verhelle D, Tartare-Deckert S, White MF, Van Obberghen E. Insulin receptor substrate-2 binds to the insulin receptor through its phosphotyrosine-binding domain and through a newly identified domain comprising amino acids 591-786. J Biol Chem. 1996 Mar 15;271(11):5980-3. DOI: 10.1074/jbc.271.11.5980.

61. White MF. The insulin signalling system and the IRS proteins. In: Diabetologia. Diabetologia; 1997. DOI: $10.1007 / \mathrm{s} 001250051387$.

62. Guo S, Dunn S, White M. The Reciprocal Stability of FOXO1 and IRS2 Creates a Regulatory Circuit that Controls Insulin Signaling. Mol Endocrinol. 2006 Dec 1;20:3389-99. DOI: 10.1210/me.2006-0092.

63. Rabiee A, Krüger M, Ardenkjær-Larsen J, Kahn CR, Emanuelli B. Distinct signalling properties of insulin receptor substrate (IRS)-1 and IRS-2 in mediating insulin/IGF-1 action. Cell Signal. 2018 Jul 1;47:1-15. DOI: 10.1016/j.cellsig.2018.03.003.

64. Tu X, Wu A, Maiorana A, Baserga R. Subcellular Localization of IRS-1 in Cell Proliferation and Differentiation. Horm Metab Res. 2003 Nov;35(11-12):734-9. DOI: 10.1055/s-2004-814155.

65. Wu A, Chen J, Baserga R. Nuclear insulin receptor substrate-1 activates promoters of cell cycle progression genes. Oncogene. 2008 Jan 10;27(3):397-403. DOI: 10.1038/sj.onc.1210636.

66. Ozoe A, Sone M, Fukushima T, Kataoka N, Chida K, Asano T, Hakuno F, Takahashi SI. Insulin receptor substrate-1 associates with small nucleolar RNA which contributes to ribosome biogenesis. Front Endocrinol (Lausanne). 2014;5(MAR). DOI: 10.3389/fendo.2014.00024.

67. Araki E, Lipes MA, Patti ME, Brüning JC, Haag B, Johnson RS, Kahn CR. Alternative pathway of insulin signalling in mice with targeted disruption of the IRS-1 gene. Nature. 1994;372(6502):186-90. DOI: $10.1038 / 372186 \mathrm{a} 0$.

68. Higaki Y, Wojtaszewski JFP, Hirshman MF, Withers DJ, Towery H, White MF, Goodyear LJ. Insulin receptor substrate-2 is not necessary for insulin- and exercise- stimulated glucose transport in skeletal muscle. $J$ Biol Chem. 1999 Jul 23;274(30):20791-5. DOI: 10.1074/jbc.274.30.20791.

69. Yamauchi T, Tobe K, Tamemoto H, Ueki K, Kaburagi Y, Yamamoto-Honda R, Takahashi Y, Yoshizawa F, Aizawa S, Akanuma Y, Sonenberg N, Yazaki Y, Kadowaki T. Insulin signalling and insulin actions in the muscles and livers of insulin-resistant, insulin receptor substrate 1-deficient mice. Mol Cell Biol. 1996 Jun;16(6):3074-84. DOI: 10.1128/mcb.16.6.3074.

70. Eckstein SS, Weigert C, Lehmann R. Divergent Roles of IRS (Insulin Receptor Substrate) 1 and 2 in Liver and Skeletal Muscle. Curr Med Chem. 2017 Jul;24(17):1827-52. DOI: 10.2174/0929867324666170426142826.

71. Kleinridders A, Ferris HA, Cai W, Kahn CR. Insulin Action in Brain Regulates Systemic Metabolism and Brain Function. Diabetes. 2014 Jul;63(7):2232-43. DOI: 10.2337/db14-0568.

72. Numan S, Russell DS. Discrete expression of insulin receptor substrate-4 mRNA in adult rat brain. Mol Brain Res. 1999 Sep 8;72(1):97-102. DOI: 10.1016/S0169-328X(99)00160-6.

73. Sutton AK, Gonzalez IE, Sadagurski M, Rajala M, Lu C, Allison MB, Adams JM, Myers MG, White MF, Olson DP. Paraventricular, subparaventricular and periventricular hypothalamic IRS4-expressing neurons are required for normal energy balance. Sci Rep. 2020 Dec 1;10(1). DOI: 10.1038/s41598-020-62468-z.

74. Heinen CA, De Vries EM, Alders M, Bikker H, Zwaveling-Soonawala N, Van Den Akker ELT, Bakker B, Hoorweg-Nijman G, Roelfsema F, Hennekam RC, Boelen A, Paul Van Trotsenburg AS, Fliers E. Mutations in IRS4 are associated with central hypothyroidism. J Med Genet. 2018 Oct 1;55(10):693-700. DOI: 10.1136/jmedgenet-2017-105113.

75. Yarchoan M, Toledo JB, Lee EB, Arvanitakis Z, Kazi H, Han LY, Louneva N, Lee VMY, Kim SF, Trojanowski JQ, Arnold SE. Abnormal serine phosphorylation of insulin receptor substrate 1 is associated with tau pathology in Alzheimer's disease and tauopathies. Acta Neuropathol. 2014;128(5):679-89. DOI: 10.1007/s00401-014- 
$1328-5$.

76. Talbot K, Wang H-Y, Kazi H, Han L-Y, Bakshi KP, Stucky A, Fuino RL, Kawaguchi KR, Samoyedny AJ, Wilson RS, Arvanitakis Z, Schneider JA, Wolf BA, Bennett DA, Trojanowski JQ, Arnold SE. Demonstrated brain insulin resistance in Alzheimer's disease patients is associated with IGF-1 resistance, IRS-1 dysregulation, and cognitive decline. J Clin Invest. 2012 Apr;122(4):1316-38. DOI: 10.1172/JCI59903.

77. Arvanitakis Z, Wang HY, Capuano AW, Khan A, Taïb B, Anokye-Danso F, Schneider JA, Bennett DA, Ahima RS, Arnold SE. Brain Insulin Signaling, Alzheimer Disease Pathology, and Cognitive Function. Ann Neurol. 2020 Sep 1;88(3):513-25. DOI: 10.1002/ana.25826.

78. Kapogiannis D, Boxer A, Schwartz JB, Abner EL, Biragyn A, Masharani U, Frassetto L, Petersen RC, Miller BL, Goetzl EJ. Dysfunctionally phosphorylated type 1 insulin receptor substrate in neural-derived blood exosomes of preclinical Alzheimer's disease. FASEB J. 2015 Feb 1;29(2):589-96. DOI: 10.1096/fj.14-262048.

79. Kapogiannis D, Mustapic M, Shardell MD, Berkowitz ST, Diehl TC, Spangler RD, Tran J, Lazaropoulos MP, Chawla S, Gulyani S, Eitan E, An Y, Huang CW, Oh ES, Lyketsos CG, Resnick SM, Goetzl EJ, Ferrucci L. Association of Extracellular Vesicle Biomarkers with Alzheimer Disease in the Baltimore Longitudinal Study of Aging. JAMA Neurol. 2019 Nov 1;76(11):1340-51. DOI: 10.1001/jamaneurol.2019.2462.

80. Bathina S, Das UN. Brain-derived neurotrophic factor and its clinical Implications. Vol. 11, Archives of Medical Science. Termedia Publishing House Ltd.; 2015. p. 1164-78. DOI: 10.5114/aoms.2015.56342.

81. Schubert M, Brazil DP, Burks DJ, Kushner JA, Ye J, Flint CL, Farhang-Fallah J, Dikkes P, Warot XM, Rio C, Corfas G, White MF. Insulin receptor substrate-2 deficiency impairs brain growth and promotes tau phosphorylation. J Neurosci. 2003 Aug 6;23(18):7084-92. DOI: 10.1523/jneurosci.23-18-07084.2003.

82. Martín ED, Sánchez-Perez A, Trejo JL, Martin-Aldana JA, Cano Jaimez M, Pons S, Acosta Umanzor C, Menes L, White MF, Burks DJ. IRS-2 Deficiency impairs NMDA receptor-dependent long-term potentiation. Cereb Cortex. 2012 Aug;22(8):1717-27. DOI: 10.1093/cercor/bhr216.

83. Germolec DR, Shipkowski KA, Frawley RP, Evans E. Markers of inflammation. In: Methods in Molecular Biology. Humana Press Inc.; 2018. p. 57-79. DOI: 10.1007/978-1-4939-8549-4_5.

84. Karimbux NY, Saraiya VM, Elangovan S, Allareddy V, Kinnunen T, Kornman KS, Duff GW. Interleukin-1 Gene Polymorphisms and Chronic Periodontitis in Adult Whites: A Systematic Review and Meta-Analysis. $J$ Periodontol. 2012 Nov;83(11):1407-19. DOI: 10.1902/jop.2012.110655.

85. Schultze-Florey CR, Martínez-Maza O, Magpantay L, Breen EC, Irwin MR, Gündel H, O’Connor MF. When grief makes you sick: Bereavement induced systemic inflammation is a question of genotype. Brain Behav Immun. 2012 Oct;26(7):1066-71. DOI: 10.1016/j.bbi.2012.06.009.

86. DiSabato DJ, Quan N, Godbout JP. Neuroinflammation: the devil is in the details. J Neurochem. 2016 Oct;139:136-53. DOI: 10.1111/jnc.13607.

87. Ransohoff RM, Brown MA. Innate immunity in the central nervous system. Vol. 122, Journal of Clinical Investigation. J Clin Invest; 2012. p. 1164-71. DOI: 10.1172/JCI58644.

88. Nimmerjahn A, Kirchhoff F, Helmchen F. Neuroscience: Resting microglial cells are highly dynamic surveillants of brain parenchyma in vivo. Science (80- ). 2005 May 27;308(5726):1314-8. DOI: 10.1126/science.1110647.

89. Sharma D, Kanneganti TD. The cell biology of inflammasomes: Mechanisms of inflammasome activation and regulation. Vol. 213, Journal of Cell Biology. Rockefeller University Press; 2016. p. 617-29. DOI: $10.1083 /$ jcb.201602089.

90. Anwar MA, Shah M, Kim J, Choi S. Recent clinical trends in Toll-like receptor targeting therapeutics. Vol. 39, Medicinal Research Reviews. John Wiley and Sons Inc.; 2019. p. 1053-90. DOI: 10.1002/med.21553.

91. Albornoz EA, Woodruff TM, Gordon R. Inflammasomes in CNS Diseases. Exp Suppl. 2018 Jan 1;108:41-60. DOI: 10.1007/978-3-319-89390-7_3.

92. Bagyinszky E, Giau V Van, Shim K, Suk K, An SSA, Kim SY. Role of inflammatory molecules in the Alzheimer's disease progression and diagnosis. Vol. 376, Journal of the Neurological Sciences. Elsevier B.V.; 2017. p. 242-54. DOI: 10.1016/j.jns.2017.03.031.

93. Obulesu M, Jhansilakshmi M. Neuroinflammation in Alzheimer's disease: an understanding of physiology and pathology. Int J Neurosci. 2014;124(4):227-35. DOI: 10.3109/00207454.2013.831852.

94. Tournier BB, Tsartsalis S, Ceyzériat K, Garibotto V, Millet P. In Vivo TSPO Signal and Neuroinflammation in Alzheimer's Disease. Vol. 9, Cells. NLM (Medline); 2020. DOI: 10.3390/cells9091941. 
95. Swardfager W, Lanctt K, Rothenburg L, Wong A, Cappell J, Herrmann N. A meta-analysis of cytokines in Alzheimer's disease. Biol Psychiatry. 2010 Nov 15;68(10):930-41. DOI: 10.1016/j.biopsych.2010.06.012.

96. Meda L, Cassatella MA, Szendrei GI, Otvos L, Baron P, Villalba M, Ferrari D, Rossi F. Activation of microglial cells by $\beta$-amyloid protein and interferon- $\gamma$. Nature. 1995;374(6523):647-50. DOI: 10.1038/374647a0.

97. Perea JR, Bolós M, Avila J. Microglia in Alzheimer's Disease in the Context of Tau Pathology. Biomolecules. 2020 Oct 14;10(10):1439. DOI: 10.3390/biom10101439.

98. François A, Rioux Bilan A, Quellard N, Fernandez B, Janet T, Chassaing D, Paccalin M, Terro F, Page G. Longitudinal follow-up of autophagy and inflammation in brain of APPswePS1dE9 transgenic mice. $J$ Neuroinflammation. 2014 Aug 27;11(1). DOI: 10.1186/s12974-014-0139-x.

99. Heneka MT, Kummer MP, Stutz A, Delekate A, Schwartz S, Vieira-Saecker A, Griep A, Axt D, Remus A, Tzeng TC, Gelpi E, Halle A, Korte M, Latz E, Golenbock DT. NLRP3 is activated in Alzheimer's disease and contributes to pathology in APP/PS1 mice. Nature. 2013 Jan 31;493(7434):674-8. DOI: 10.1038/nature11729.

100. Hanslik KL, Ulland TK. The Role of Microglia and the Nlrp3 Inflammasome in Alzheimer's Disease. Vol. 11, Frontiers in Neurology. Frontiers Media S.A.; 2020. DOI: 10.3389/fneur.2020.570711.

101. Feng YS, Tan ZX, Wu LY, Dong F, Zhang F. The involvement of NLRP3 inflammasome in the treatment of Alzheimer's disease. Vol. 64, Ageing Research Reviews. Elsevier Ireland Ltd; 2020. DOI: 10.1016/j.arr.2020.101192.

102. Clement A, Wiborg O, Asuni AA. Steps Towards Developing Effective Treatments for Neuropsychiatric Disturbances in Alzheimer's Disease: Insights From Preclinical Models, Clinical Data, and Future Directions. Vol. 12, Frontiers in Aging Neuroscience. Frontiers Media S.A.; 2020. DOI: 10.3389/fnagi.2020.00056.

103. McGeer PL, Rogers J, McGeer EG. Inflammation, Antiinflammatory Agents, and Alzheimer's Disease: The Last 22 Years. Vol. 54, Journal of Alzheimer's Disease. IOS Press; 2016. p. 853-7. DOI: 10.3233/JAD-160488.

104. Pasinetti GM. From epidemiology to therapeutic trials with anti-inflammatory drugs in Alzheimer's disease: The role of NSAIDs and cyclooxygenase in $\beta$-amyloidosis and clinical dementia. Vol. 4, Journal of Alzheimer's Disease. IOS Press; 2002. p. 435-45. DOI: 10.3233/JAD-2002-4510.

105. Meyer PF, Tremblay-Mercier J, Leoutsakos J, Madjar C, Lafaille-Maignan MÉ, Savard M, Rosa-Neto P, Poirier J, Etienne P, Breitner J. INTREPAD: A randomized trial of naproxen to slow progress of presymptomatic Alzheimer disease. Neurology. 2019 Apr 30;92(18):e2070-80. DOI: 10.1212/WNL.0000000000007232.

106. Breitner J, Baker L, Drye L, Evans D, Lyketsos CG, Ryan L, Zandi P, Saucedo HH, Anau J, Cholerton B. Follow-up evaluation of cognitive function in the randomized Alzheimer's disease anti-inflammatory prevention trial and its follow-up study. Alzheimer's Dement. 2015;11(2):216-225.e1. DOI: 10.1016/j.jalz.2014.03.009.

107. Butchart J, Brook L, Hopkins V, Teeling J, Püntener U, Culliford D, Sharples R, Sharif S, McFarlane B, Raybould R, Thomas R, Passmore P, Perry VH, Holmes C. Etanercept in Alzheimer disease. Neurology. 2015 May 26;84(21):2161-8. DOI: 10.1212/WNL.0000000000001617.

108. Najem D, Bamji-Mirza M, Chang N, Liu QY, Zhang W. Insulin resistance, neuroinflammation, and Alzheimer's disease. Vol. 25, Reviews in the Neurosciences. Walter de Gruyter GmbH; 2014. p. 509-25. DOI: 10.1515/revneuro-2013-0050.

109. Sánchez-Sarasúa S, Fernández-Pérez I, Espinosa-Fernández V, Sánchez-Pérez AM, Ledesma JC. Can we treat neuroinflammation in alzheimer's disease?. Vol. 21, International Journal of Molecular Sciences. MDPI AG; 2020. p. 1-23. DOI: $10.3390 /$ ijms 21228751 .

110. Mielke JG, Taghibiglou C, Wang YT. Endogenous insulin signaling protects cultured neurons from oxygenglucose deprivation-induced cell death. Neuroscience. 2006 Nov 17;143(1):165-73. DOI: 10.1016/j.neuroscience.2006.07.055.

111. Chiu SL, Chen CM, Cline HT. Insulin Receptor Signaling Regulates Synapse Number, Dendritic Plasticity, and Circuit Function In Vivo. Neuron. 2008 Jun 12;58(5):708-19. DOI: 10.1016/j.neuron.2008.04.014.

112. Dou JT, Chen M, Dufour F, Alkon DL, Zhao WQ. Insulin receptor signaling in long-term memory consolidation following spatial learning. Learn Mem. 2005 Nov;12(6):646-55. DOI: 10.1101/lm.88005.

113. Chen L, Chen R, Wang H, Liang F. Mechanisms Linking Inflammation to Insulin Resistance. Vol. 2015, International Journal of Endocrinology. Hindawi Limited; 2015. DOI: 10.1155/2015/508409.

114. Ribes-Navarro A, Atef M, Sánchez-Sarasúa S, Beltrán-Bretones MT, Olucha-Bordonau F, Sánchez-Pérez AM. Abscisic Acid Supplementation Rescues High Fat Diet-Induced Alterations in Hippocampal Inflammation and IRSs Expression. Mol Neurobiol. 2018 May 2; DOI: 10.1007/s12035-018-1091-z. 
115. M. de la Monte S. Brain Insulin Resistance and Deficiency as Therapeutic Targets in Alzheimers Disease. Curr Alzheimer Res. 2012 Jan 18;9(1):35-66. DOI: 10.2174/156720512799015037.

116. Hameed I, Masoodi SR, Mir SA, Nabi M, Ghazanfar K, Ganai BA. Type 2 diabetes mellitus: From a metabolic disorder to an inflammatory condition. World J Diabetes. 2015;6(4):598. DOI: 10.4239/wjd.v6.i4.598.

117. Cai D, Liu T. Hypothalamic inflammation: a double-edged sword to nutritional diseases. Vol. 1243, Annals of the New York Academy of Sciences. Ann N Y Acad Sci; 2011. DOI: 10.1111/j.1749-6632.2011.06388.x.

118. Sánchez-Sarasúa S, Moustafa S, García-Avilés Á, López-Climent MF, Gómez-Cadenas A, Olucha-Bordonau FE, Sánchez-Pérez AM. The effect of abscisic acid chronic treatment on neuroinflammatory markers and memory in a rat model of high-fat diet induced neuroinflammation. Nutr Metab (Lond). 2016 Dec 26;13(1):73. DOI: $10.1186 / \mathrm{s} 12986-016-0137-3$.

119. Chen W, Balland E, Cowley MA. Hypothalamic Insulin Resistance in Obesity: Effects on Glucose Homeostasis. Vol. 104, Neuroendocrinology. S. Karger AG; 2017. p. 364-81. DOI: 10.1159/000455865.

120. Kandimalla R, Thirumala V, Reddy PH. Is Alzheimer's disease a Type 3 Diabetes? A critical appraisal. Vol. 1863, Biochimica et Biophysica Acta - Molecular Basis of Disease. Elsevier B.V.; 2017. p. 1078-89. DOI: 10.1016/j.bbadis.2016.08.018.

121. Moloney AM, Griffin RJ, Timmons S, O'Connor R, Ravid R, O'Neill C. Defects in IGF-1 receptor, insulin receptor and IRS-1/2 in Alzheimer's disease indicate possible resistance to IGF-1 and insulin signalling. Neurobiol Aging. 2010 Feb;31(2):224-43. DOI: 10.1016/j.neurobiolaging.2008.04.002.

122. Steen E, Terry BM, Rivera EJ, Cannon JL, Neely TR, Tavares R, Xu XJ, Wands JR, De La Monte SM. Impaired insulin and insulin-like growth factor expression and signaling mechanisms in Alzheimer's disease - Is this type 3 diabetes? J Alzheimer's Dis. 2005;7(1):63-80. DOI: 10.3233/JAD-2005-7107.

123. Rivera EJ, Goldin A, Fulmer N, Tavares R, Wands JR, De La Monte SM. Insulin and insulin-like growth factor expression and function deteriorate with progression of Alzheimer's disease: Link to brain reductions in acetylcholine. J Alzheimer's Dis. 2005;8(3):247-68. DOI: 10.3233/JAD-2005-8304.

124. Talbot K, Wang H, Kazi H, Han L, Bakshi KP, Stucky A, Fuino RL, Kawaguchi KR, Samoyedny AJ, Wilson RS, Arvanitakis Z, Schneider J a, Wolf B a, Bennett D a, Trojanowski JQ, Arnold SE. Demonstrated brain insulin resistance in alzheimer's disease patients is assocaited with IGF-1 resisitance, IRS-1 dysregulation, and cogntive decline. J Clin Invest. 2012;122(4). DOI: 10.1172/JCI59903DS1.

125. Lv H, Tang L, Guo C, Jiang Y, Gao C, Wang Y, Jian C. Intranasal insulin administration may be highly effective in improving cognitive function in mice with cognitive dysfunction by reversing brain insulin resistance. Cogn Neurodyn. 2020 Jun 1;14(3):323-38. DOI: 10.1007/s11571-020-09571-z.

126. Mishra SK, Hidau M. Intranasal Insulin Enhances Intracerebroventricular Streptozotocin-Induced Decrease in Olfactory Discriminative Learning via Upregulation of Subventricular Zone-Olfactory Bulb Neurogenesis in the Rat Model. Mol Neurobiol. 2020; DOI: 10.1007/s12035-020-02185-w.

127. Farzampour S, Majdi A, Sadigh-Eteghad S. Intranasal insulin treatment improves memory and learning in a rat amyloid-beta model of Alzheimer's disease. Acta Physiol Hung. 2016 Sep 1;103(3):344-53. DOI: 10.1556/2060.103.2016.3.7.

128. Guo Z, Chen Y, Mao YF, Zheng T, Jiang Y, Yan Y, Yin X, Zhang B. Long-term treatment with intranasal insulin ameliorates cognitive impairment, tau hyperphosphorylation, and microglial activation in a streptozotocin-induced Alzheimer's rat model. Sci Rep. 2017 Apr 6;7. DOI: 10.1038/srep45971.

129. Avgerinos KI, Kalaitzidis G, Malli A, Kalaitzoglou D, Myserlis PG, Lioutas VA. Intranasal insulin in Alzheimer's dementia or mild cognitive impairment: a systematic review. Vol. 265, Journal of Neurology. Dr. Dietrich Steinkopff Verlag GmbH and Co. KG; 2018. p. 1497-510. DOI: 10.1007/s00415-018-8768-0.

130. Thankappan S, Sen S, Subramanian S, Sinha P, Purushottam M, Bharath S. Insulin resistance in patients with Alzheimer's dementia: A controlled study from India. Vol. 38, Asian Journal of Psychiatry. Elsevier B.V.; 2018. p. 33-4. DOI: 10.1016/j.ajp.2018.10.026.

131. Koenig AM, Mechanic-Hamilton D, Xie SX, Combs MF, Cappola AR, Xie L, Detre JA, Wolk DA, Arnold SE. Effects of the Insulin Sensitizer Metformin in Alzheimer Disease: Pilot Data From a Randomized Placebocontrolled Crossover Study. Alzheimer Dis Assoc Disord. 2017 May;31(2):107-13. DOI: 10.1097/WAD.0000000000000202.

132. Salminen A, Kaarniranta K, Haapasalo A, Soininen H, Hiltunen M. AMP-activated protein kinase: A potential player in Alzheimer's disease. Vol. 118, Journal of Neurochemistry. J Neurochem; 2011. p. 460-74. DOI: 10.1111/j.1471-4159.2011.07331.x. 
133. Eagles CF, Wareing PF. Dormancy regulators in woody plants: Experimental induction of dormancy in Betula pubescens. Nature. 1963;199(4896):874-5. DOI: 10.1038/199874a0.

134. Ohkuma K, Lyon JL, Addicott FT, Smith OE. Abscisin II, an abscission-accelerating substance from young cotton fruit. Science (80- ). 1963;142(3599):1592-3. DOI: 10.1126/science.142.3599.1592.

135. Addicott FT, Lyon JL, Ohkuma K, Thiessen WE, Carns HR, Smith OE, Cornforth JW, Milborrow B V., Ryback G, Wareing PF. Abscisic acid: A new name for Abscisin II (Dormin). Science (80- ). 1968 Mar 29;159(3822):1493. DOI: 10.1126/science.159.3822.1493.

136. Cornforth JW, Milborrow B V., Ryback G, Wareing PF. Chemistry and physiology of "dormins" in sycamore: Identity of sycamore "dormin" with abscisin II. Nature. 1965;205(4978):1269-70. DOI: 10.1038/2051269b0.

137. Chen K, Li GJ, Bressan RA, Song CP, Zhu JK, Zhao Y. Abscisic acid dynamics, signaling, and functions in plants. Vol. 62, Journal of Integrative Plant Biology. Blackwell Publishing Ltd; 2020. p. 25-54. DOI: $10.1111 /$ jipb.12899.

138. Chen JG, Ellis BE. GCR2 is a new member of the eukaryotic lanthionine synthetase component C-like protein family. Plant Signal Behav. 2008;3(5):307-10. DOI: 10.4161/psb.3.5.5292.

139. Olds CL, Glennon EKK, Luckhart S. Abscisic acid: new perspectives on an ancient universal stress signaling molecule. Microbes Infect. 2018 Oct 1;20(9-10):484-92. DOI: 10.1016/j.micinf.2018.01.009.

140. Sturla L, Fresia C, Guida L, Bruzzone S, Scarfì S, Usai C, Fruscione F, Magnone M, Millo E, Basile G, Grozio A, Jacchetti E, Allegretti M, De Flora A, Zocchi E. LANCL2 is necessary for abscisic acid binding and signaling in human granulocytes and in rat insulinoma cells. J Biol Chem. 2009 Oct 9;284(41):28045-57. DOI: 10.1074/jbc.M109.035329.

141. Hamm JK, El Jack AK, Pilch PF, Farmer SR. Role of PPAR $\gamma$ in regulating adipocyte differentiation and insulinresponsive glucose uptake. In: Annals of the New York Academy of Sciences. New York Academy of Sciences; 1999. p. 134-45. DOI: 10.1111/j.1749-6632.1999.tb07792.x.

142. Auwerx J, Cock T-A, Knouff C. PPAR- $\gamma$ : A Thrifty Transcription Factor. Nucl Recept Signal. 2003 Jan;1(1):nrs.01006. DOI: 10.1621/nrs.01006.

143. Armoni M, Harel C, Karnieli E. Transcriptional regulation of the GLUT4 gene: from PPAR- $\gamma$ and FOXO1 to FFA and inflammation. Trends Endocrinol Metab. 2007 Apr;18(3):100-7. DOI: 10.1016/j.tem.2007.02.001.

144. Hirukawa H, Kaneto H, Shimoda M, Kimura T, Okauchi S, Obata A, Kohara K, Hamamoto S, Tawaramoto K, Hashiramoto M, Kaku K. Combination of DPP-4 inhibitor and PPAR $\gamma$ agonist exerts protective effects on pancreatic $\beta$-cells in diabetic $\mathrm{db} / \mathrm{db}$ mice through the augmentation of IRS-2 expression. Mol Cell Endocrinol. 2015;413:49-60. DOI: 10.1016/j.mce.2015.06.010.

145. Xie Z, Ruas P, Shen QJ. Regulatory Networks of the Phytohormone Abscisic Acid. Vol. 72, Vitamins and Hormones. Vitam Horm; 2005. p. 235-69. DOI: 10.1016/S0083-6729(05)72007-0.

146. Guri AJ, Hontecillas R, Bassaganya-Riera J. Abscisic acid synergizes with rosiglitazone to improve glucose tolerance and down-modulate macrophage accumulation in adipose tissue: Possible action of the cAMP/PKA/PPAR $\gamma$ axis. Clin Nutr. 2010 Oct;29(5):646-53. DOI: 10.1016/j.clnu.2010.02.003.

147. Bassaganya-Riera J, Guri AJ, Lu P, Climent M, Carbo A, Sobral BW, Horne WT, Lewis SN, Bevan DR, Hontecillas R. Abscisic acid regulates inflammation via ligand-binding domain-independent activation of peroxisome proliferator-activated receptor gamma. J Biol Chem. 2011 Jan 28;286(4):2504-16. DOI: 10.1074/jbc.M110.160077.

148. Bruzzone S, Bodrato N, Usai C, Guida L, Moreschi I, Nano R, Antonioli B, Fruscione F, Magnone M, Scarfî S, De Flora A, Zocchi E. Abscisic acid is an endogenous stimulator of insulin release from human pancreatic islets with cyclic ADP ribose as second messenger. J Biol Chem. 2008 Nov 21;283(47):32188-97. DOI: 10.1074/jbc.M802603200.

149. Bruzzone S, Moreschi I, Usai C, Guida L, Damonte G, Salis A, Scarfi S, Millo E, De Flora A, Zocchi E. Abscisic acid is an endogenous cytokine in human granulocytes with cyclic ADP-ribose as second messenger. Proc Natl Acad Sci U S A. 2007 Apr 3;104(14):5759-64. DOI: 10.1073/pnas.0609379104.

150. Ameri P, Bruzzone S, Mannino E, Sociali G, Andraghetti G, Salis A, Ponta ML, Briatore L, Adami GF, Ferraiolo A, Venturini PL, Maggi D, Cordera R, Murialdo G, Zocchi E. Impaired increase of plasma abscisic acid in response to oral glucose load in type 2 diabetes and in gestational diabetes. PLoS One. 2015 Feb 27;10(2). DOI: 10.1371/journal.pone.0115992.

151. Guri AJ, Hontecillas R, Si H, Liu D, Bassaganya-Riera J. Dietary abscisic acid ameliorates glucose tolerance 
and obesity-related inflammation in db/db mice fed high-fat diets. Clin Nutr. 2007 Feb;26(1):107-16. DOI: 10.1016/j.clnu.2006.07.008.

152. Magnone M, Ameri P, Salis A, Andraghetti G, Emionite L, Murialdo G, De Flora A, Zocchi E. Microgram amounts of abscisic acid in fruit extracts improve glucose tolerance and reduce insulinemia in rats and in humans. FASEB J. 2015 Dec;29(12):4783-93. DOI: 10.1096/fj.15-277731.

153. Atkinson FS, Villar A, Mulà A, Zangara A, Risco E, Smidt CR, Hontecillas R, Leber A, Bassaganya-Riera J. Abscisic acid standardized fig (Ficus carica) extracts ameliorate postprandial glycemic and insulinemic responses in healthy adults. Nutrients. 2019 Aug 1;11(8). DOI: 10.3390/nu11081757.

154. Bruzzone S, Magnone M, Mannino E, Sociali G, Sturla L, Fresia C, Booz V, Emionite L, De Flora A, Zocchi E. Abscisic acid stimulates glucagon-like peptide-1 secretion from L-cells and its oral administration increases plasma glucagon-like peptide-1 levels in rats. PLoS One. 2015;10(10). DOI: 10.1371/journal.pone.0140588.

155. Bruzzone S, Ameri P, Briatore L, Mannino E, Basile G, Andraghetti G, Grozio A, Magnone M, Guida L, Scarfì S, Salis A, Damonte G, Sturla L, Nencioni A, Fenoglio D, Fiory F, Miele C, Beguinot F, Ruvolo V, Bormioli M, Colombo G, Maggi D, Murialdo G, Cordera R, De Flora A, Zocchi E. The plant hormone abscisic acid increases in human plasma after hyperglycemia and stimulates glucose consumption by adipocytes and myoblasts. FASEB J. 2012 Mar;26(3):1251-60. DOI: 10.1096/fj.11-190140.

156. Scarfi S, Ferraris C, Fruscione F, Fresia C, Guida L, Bruzzone S, Usai C, Parodi A, Millo E, Salis A, Burastero G, De Flora A, Zocchi E. Cyclic ADP-Ribose-Mediated Expansion and Stimulation of Human Mesenchymal Stem Cells by the Plant Hormone Abscisic Acid. Stem Cells. 2008 Nov;26(11):2855-64. DOI: 10.1634/stemcells.2008-0488.

157. Scarfi S, Fresia C, Ferraris C, Bruzzone S, Fruscione F, Usai C, Benvenuto F, Magnone M, Podestà M, Sturla L, Guida L, Albanesi E, Damonte G, Salis A, De Flora A, Zocchi E. The plant hormone abscisic acid stimulates the proliferation of human hemopoietic progenitors through the second messenger cyclic ADP-ribose. Stem Cells. 2009 Oct;27(10):2469-77. DOI: 10.1002/stem.173.

158. Zhou N, Yao Y, Ye H, Zhu W, Chen L, Mao Y. Abscisic-acid-induced cellular apoptosis and differentiation in glioma via the retinoid acid signaling pathway. Int $J$ Cancer. 2016 Apr 15;138(8):1947-58. DOI: 10.1002/ijc.29935.

159. Magnone M, Bruzzone S, Guida L, Damonte G, Millo E, Scarfì S, Usai C, Sturla L, Palombo D, De Flora A, Zocchi E. Abscisic acid released by human monocytes activates monocytes and vascular smooth muscle cell responses involved in atherogenesis. $J$ Biol Chem. 2009 Jun 26;284(26):17808-18. DOI: 10.1074/jbc.M809546200.

160. Bouhlel MA, Derudas B, Rigamonti E, Dièvart R, Brozek J, Haulon S, Zawadzki C, Jude B, Torpier G, Marx N, Staels B, Chinetti-Gbaguidi G. PPARgamma activation primes human monocytes into alternative M2 macrophages with anti-inflammatory properties. Cell Metab. 2007 Aug;6(2):137-43. DOI: 10.1016/j.cmet.2007.06.010.

161. Qi C-C, Ge J-F, Zhou J-N. Preliminary evidence that abscisic acid improves spatial memory in rats. Physiol Behav. 2015 Feb;139:231-9. DOI: 10.1016/j.physbeh.2014.11.053.

162. Qi C-C, Zhang Z, Fang H, Liu J, Zhou N, Ge J-F, Chen F-H, Xiang C-B, Zhou J-N. Antidepressant effects of abscisic acid mediated by the downregulation of corticotrophin-releasing hormone gene expression in rats. Int $J$ Neuropsychopharmacol. 2015 Feb;18(4). DOI: 10.1093/ijnp/pyu006.

163. Espinosa-Fernández V, Mañas-Ojeda A, Pacheco-Herrero M, Castro-Salazar E, Ros-Bernal F, Sánchez-Pérez AM. Early intervention with ABA prevents neuroinflammation and memory impairment in a triple transgenic mice model of Alzheimer's disease. Behav Brain Res. 2019 Nov 18;374:112106. DOI: 10.1016/j.bbr.2019.112106.

164. Jeon SH, Kim N, Ju YJ, Gee MS, Lee D, Lee JK. Phytohormone abscisic acid improves memory impairment and reduces neuroinflammation in 5XFAD mice by upregulation of lanc-like protein 2. Int J Mol Sci. 2020 Nov 2;21(22):1-14. DOI: 10.3390/ijms21228425.

165. Khorasani A, Abbasnejad M, Esmaeili-Mahani S. Phytohormone abscisic acid ameliorates cognitive impairments in streptozotocin-induced rat model of Alzheimer's disease through PPAR $\beta / \delta$ and PKA signaling. Int J Neurosci. 2019 Nov 2;129(11):1053-65. DOI: 10.1080/00207454.2019.1634067.

166. Zhang JM, An J. Cytokines, inflammation, and pain. Vol. 45, International Anesthesiology Clinics. Int Anesthesiol Clin; 2007. p. 27-37. DOI: 10.1097/AIA.0b013e318034194e.

167. Mollashahi M, Abbasnejad M, Esmaeili-Mahani S. Spinal protein kinase A and phosphorylated extracellular signal-regulated kinase signaling are involved in the antinociceptive effect of phytohormone abscisic acid in rats. 
Arq Neuropsiquiatr. 2020;78(1):21-7. DOI: 10.1590/0004-282X20190137.

168. Mollashahi M, Abbasnejad M, Esmaeili-Mahani S. Phytohormone abscisic acid elicits antinociceptive effects in rats through the activation of opioid and peroxisome proliferator-activated receptors $\beta / \delta$. Eur J Pharmacol. 2018 Aug 5;832:75-80. DOI: 10.1016/j.ejphar.2018.05.013.

169. Eichenbaum H. What H.M. taught Us. J Cogn Neurosci. 2013;25(1):14-21. DOI: 10.1162/jocn_a_00285.

170. Davachi L. Item, context and relational episodic encoding in humans. Vol. 16, Current Opinion in Neurobiology. Curr Opin Neurobiol; 2006. p. 693-700. DOI: 10.1016/j.conb.2006.10.012.

171. Suzuki WA, Naya Y. The perirhinal cortex. Vol. 37, Annual Review of Neuroscience. Annual Reviews Inc.; 2014. p. 39-53. DOI: 10.1146/annurev-neuro-071013-014207.

172. Yukie M. Connections between the medial temporal cortex and the CA1 subfield of the hippocampal formation in the Japanese monkey (Macaca fuscata). J Comp Neurol. 2000 Jul 24;423(2):282-98. DOI: 10.1002/10969861(20000724)423:2<282::AID-CNE7>3.0.CO;2-Z.

173. Sun Y, Jin S, Lin X, Chen L, Qiao X, Jiang L, Zhou P, Johnston KG, Golshani P, Nie Q, Holmes TC, Nitz DA, $\mathrm{Xu}$ X. CA1-projecting subiculum neurons facilitate object-place learning. Nat Neurosci. 2019 Nov 1;22(11):1857-70. DOI: 10.1038/s41593-019-0496-y.

174. Moser EI, Moser MB, McNaughton BL. Spatial representation in the hippocampal formation: A history. Vol. 20, Nature Neuroscience. Nature Publishing Group; 2017. p. 1448-64. DOI: 10.1038/nn.4653.

175. Wang C, Chen X, Lee H, Deshmukh SS, Yoganarasimha D, Savelli F, Knierim JJ. Egocentric coding of external items in the lateral entorhinal cortex. Science (80- ). 2018 Nov 23;362(6417):945-9. DOI: 10.1126/science.aau4940.

176. Hafting T, Fyhn M, Molden S, Moser MB, Moser EI. Microstructure of a spatial map in the entorhinal cortex. Nature. 2005 Aug 11;436(7052):801-6. DOI: 10.1038/nature03721.

177. Moser MB, Rowland DC, Moser EI. Place cells, grid cells, and memory. Cold Spring Harb Perspect Biol. 2015;7(2). DOI: 10.1101/cshperspect.a021808.

178. O'Keefe J, Speakman A. Single unit activity in the rat hippocampus during a spatial memory task. Exp Brain Res. 1987 Sep;68(1):1-27. DOI: 10.1007/BF00255230.

179. Aimone JB, Deng W, Gage FH. Resolving New Memories: A Critical Look at the Dentate Gyrus, Adult Neurogenesis, and Pattern Separation. Vol. 70, Neuron. Neuron; 2011. p. 589-96. DOI: 10.1016/j.neuron.2011.05.010.

180. Yuan M, Meyer T, Benkowitz C, Savanthrapadian S, Ansel-Bollepalli L, Foggetti A, Wulff P, Alcami P, Elgueta C, Bartos M. Somatostatin-positive interneurons in the dentate gyrus of mice provide local- and longrange septal synaptic inhibition. Elife. 2017 Apr 3;6. DOI: 10.7554/eLife.21105.

181. Khakpai F, Nasehi M, Haeri-Rohani A, Eidi A, Zarrindast MR. Septo-hippocampo-septal loop and memory formation. Basic Clin Neurosci. 2013;4(1):5-23.

182. Borhegyi Z, Freund TF. Dual projection from the medial septum to the supramammillary nucleus in the rat. Brain Res Bull. 1998 Jul 15;46(5):453-9. DOI: 10.1016/S0361-9230(98)00038-0.

183. Tsivilis D, Vann SD, Denby C, Roberts N, Mayes AR, Montaldi D, Aggleton JP. A disproportionate role for the fornix and mammillary bodies in recall versus recognition memory. Nat Neurosci. 2008 Jul;11(7):834-42. DOI: $10.1038 / \mathrm{nn} .2149$.

184. Aggleton JP, Brown MW. Episodic memory, amnesia, and the hippocampal-anterior thalamic axis. Behav Brain Sci. 1999 Jun;22(3):425-89.

185. Vertes RP, Albo Z, Viana Di Prisco G. Theta-rhythmically firing neurons in the anterior thalamus: Implications for mnemonic functions of Papez's circuit. Neuroscience. 2001 Jun 14;104(3):619-25. DOI: 10.1016/S03064522(01)00131-2.

186. Hornberger M, Wong S, Tan R, Irish M, Piguet O, Kril J, Hodges JR, Halliday G. In vivo and post-mortem memory circuit integrity in frontotemporal dementia and Alzheimer's disease. Brain. 2012;135(10):3015-25. DOI: 10.1093/brain/aws239.

187. Aggleton JP, Pralus A, Nelson AJD, Hornberger M. Thalamic pathology and memory loss in early Alzheimer's disease: Moving the focus from the medial temporal lobe to Papez circuit. Vol. 139, Brain. Oxford University Press; 2016. p. 1877-90. DOI: 10.1093/brain/aww083. 
188. Tan RH, Wong S, Kril JJ, Piguet O, Hornberger M, Hodges JR, Halliday GM. Beyond the temporal pole: Limbic memory circuit in the semantic variant of primary progressive aphasia. Brain. 2014;137(7):2065-76. DOI: 10.1093/brain/awu118.

189. Chen XQ, Mobley WC. Exploring the pathogenesis of Alzheimer disease in basal forebrain cholinergic neurons:Converging insights from alternative hypotheses. Vol. 13, Frontiers in Neuroscience. Frontiers Media S.A.; 2019. DOI: 10.3389/fnins.2019.00446.

190. Leung L, Andrews-Zwilling Y, Yoon SY, Jain S, Ring K, Dai J, Wang MM, Tong L, Walker D, Huang Y. Apolipoprotein E4 Causes Age- and Sex-Dependent Impairments of Hilar GABAergic Interneurons and Learning and Memory Deficits in Mice. PLoS One. 2012 Dec 31;7(12). DOI: 10.1371/journal.pone.0053569.

191. Shetty AK, Bates A. Potential of GABA-ergic cell therapy for schizophrenia, neuropathic pain, and Alzheimers and Parkinsons diseases. Vol. 1638, Brain Research. Elsevier B.V.; 2016. p. 74-87. DOI: 10.1016/j.brainres.2015.09.019.

192. Lu M-H, Zhao X-Y, Xu D-E, Chen J-B, Ji W-L, Huang Z-P, Pan T-T, Xue L-L, Wang F, Li Q-F, Zhang Y, Wang T-H, Yanagawa Y, Liu C-F, Xu R-X, Xia Y-Y, Li S, Ma Q-H. Transplantation of GABAergic Interneuron Progenitor Attenuates Cognitive Deficits of Alzheimer's Disease Model Mice. J Alzheimer's Dis. 2020 Apr 7;75(1):245-60. DOI: $10.3233 / \mathrm{jad}-200010$.

193. Xu Y, Zhao M, Han Y, Zhang H. GABAergic Inhibitory Interneuron Deficits in Alzheimer's Disease: Implications for Treatment. Vol. 14, Frontiers in Neuroscience. Frontiers Media S.A.; 2020. DOI: 10.3389/fnins.2020.00660.

194. Miller AA, Spencer SJ. Obesity and neuroinflammation: a pathway to cognitive impairment. Brain Behav Immun. 2014 Nov;42:10-21. DOI: 10.1016/j.bbi.2014.04.001.

195. Della-Morte D, Palmirotta R, Rehni AK, Pastore D, Capuani B, Pacifici F, De Marchis ML, Dave KR, Bellia A, Fogliame G, Ferroni P, Donadel G, Cacciatore F, Abete P, Dong C, Pileggi A, Roselli M, Ricordi C, Sbraccia P, Guadagni F, Rundek T, Lauro D. Pharmacogenomics and pharmacogenetics of thiazolidinediones: role in diabetes and cardiovascular risk factors. Pharmacogenomics. 2014 Dec;15(16):2063-82. DOI: 10.2217/pgs.14.162.

196. Panicker GK, Karnad DR, Salvi V, Kothari S. Cardiovascular risk of oral antidiabetic drugs: current evidence and regulatory requirements for new drugs. J Assoc Physicians India. 2012 Jan;60:56-61.

197. Nissen SE, Wolski K. Effect of rosiglitazone on the risk of myocardial infarction and death from cardiovascular causes. N Engl J Med. 2007 Jun 14;356(24):2457-71. DOI: 10.1056/NEJMoa072761.

198. Le Page-Degivry MT, Bidard JN, Rouvier E, Bulard C, Lazdunski M. Presence of abscisic acid, a phytohormone, in the mammalian brain. Proc Natl Acad Sci U S A. 1986 Feb;83(4):1155-8.

199. Minorsky P V. Abscisic acid: a universal signalling factor? Plant Physiol. 2002 Mar;128(3):788-9.

200. Gomez-Cadenas A, Vives V, Zandalinas SI, Manzi M, Sanchez-Perez AM, Perez-Clemente RM, Arbona V. Abscisic Acid: a versatile phytohormone in plant signaling and beyond. Curr Protein Pept Sci. 2015 Jan;16(5):413-34.

201. Puce S, Basile G, Bavestrello G, Bruzzone S, Cerrano C, Giovine M, Arillo A, Zocchi E. Abscisic acid signaling through cyclic ADP-ribose in hydroid regeneration. J Biol Chem. 2004 Sep 17;279(38):39783-8. DOI: 10.1074/jbc.M405348200.

202. Bassaganya-Riera J, Skoneczka J, Kingston DGJ, Krishnan A, Misyak SA, Guri AJ, Pereira A, Carter AB, Minorsky P, Tumarkin R, Hontecillas R. Mechanisms of action and medicinal applications of abscisic Acid. Curr Med Chem. 2010 Jan;17(5):467-78.

203. Guri AJ, Evans NP, Hontecillas R, Bassaganya-Riera J. T cell PPAR $\gamma$ is required for the anti-inflammatory efficacy of abscisic acid against experimental IBD. J Nutr Biochem. 2011 Sep;22(9):812-9. DOI: 10.1016/j.jnutbio.2010.06.011.

204. Lu P, Hontecillas R, Philipson CW, Bassaganya-Riera J. Lanthionine synthetase component C-like protein 2: a new drug target for inflammatory diseases and diabetes. Curr Drug Targets. 2014 Jun;15(6):565-72.

205. Bremner JD, Shearer KD, McCaffery PJ. Retinoic acid and affective disorders: the evidence for an association. $J$ Clin Psychiatry. 2012 Jan;73(1):37-50. DOI: 10.4088/JCP.10r05993.

206. Gao Y, Ottaway N, Schriever SC, Legutko B, García-Cáceres C, de la Fuente E, Mergen C, Bour S, Thaler JP, Seeley RJ, Filosa J, Stern JE, Perez-Tilve D, Schwartz MW, Tschöp MH, Yi C-X. Hormones and diet, but not body weight, control hypothalamic microglial activity. Glia. 2014 Jan;62(1):17-25. DOI: 10.1002/glia.22580. 
207. Spielman LJ, Little JP, Klegeris A. Inflammation and insulin/IGF-1 resistance as the possible link between obesity and neurodegeneration. $J$ Neuroimmunol. 2014 Aug 15;273(1-2):8-21. DOI: 10.1016/j.jneuroim.2014.06.004.

208. Sobesky JL, Barrientos RM, De May HS, Thompson BM, Weber MD, Watkins LR, Maier SF. High-fat diet consumption disrupts memory and primes elevations in hippocampal IL-1 $\beta$, an effect that can be prevented with dietary reversal or IL-1 receptor antagonism. Brain Behav Immun. 2014 Nov;42:22-32. DOI: 10.1016/j.bbi.2014.06.017.

209. Ropelle ER, Flores MB, Cintra DE, Rocha GZ, Pauli JR, Morari J, de Souza CT, Moraes JC, Prada PO, Guadagnini D, Marin RM, Oliveira AG, Augusto TM, Carvalho HF, Velloso LA, Saad MJA, Carvalheira JBC. IL-6 and IL-10 anti-inflammatory activity links exercise to hypothalamic insulin and leptin sensitivity through IKKbeta and ER stress inhibition. PLoS Biol. 2010 Jan;8(8). DOI: 10.1371/journal.pbio.1000465.

210. Gaskin S, Tardif M, Cole E, Piterkin P, Kayello L, Mumby DG. Object familiarization and novel-object preference in rats. Behav Processes. 2010 Jan;83(1):61-71. DOI: 10.1016/j.beproc.2009.10.003.

211. André C, Dinel A-L, Ferreira G, Layé S, Castanon N. Diet-induced obesity progressively alters cognition, anxiety-like behavior and lipopolysaccharide-induced depressive-like behavior: focus on brain indoleamine 2,3dioxygenase activation. Brain Behav Immun. 2014 Oct;41:10-21. DOI: 10.1016/j.bbi.2014.03.012.

212. Durgbanshi A, Arbona V, Pozo O, Miersch O, Sancho J V, Gómez-Cadenas A. Simultaneous determination of multiple phytohormones in plant extracts by liquid chromatography-electrospray tandem mass spectrometry. $J$ Agric Food Chem. 2005 Nov 2;53(22):8437-42. DOI: 10.1021/jf050884b.

213. Cui Y, Shu Y, Zhu Y, Shi Y, Le G. High-fat diets impair spatial learning of mice in the Y-maze paradigm: ameliorative potential of $\alpha$-lipoic acid. J Med Food. 2012 Aug;15(8):713-7. DOI: 10.1089/jmf.2011.1970.

214. Edwards LM, Murray AJ, Holloway CJ, Carter EE, Kemp GJ, Codreanu I, Brooker H, Tyler DJ, Robbins PA, Clarke K. Short-term consumption of a high-fat diet impairs whole-body efficiency and cognitive function in sedentary men. FASEB J. 2011 Mar;25(3):1088-96. DOI: 10.1096/fj.10-171983.

215. Ito D, Imai Y, Ohsawa K, Nakajima K, Fukuuchi Y, Kohsaka S. Microglia-specific localisation of a novel calcium binding protein, Iba1. Brain Res Mol Brain Res. 1998 Jun 1;57(1):1-9.

216. De Souza CT, Araujo EP, Bordin S, Ashimine R, Zollner RL, Boschero AC, Saad MJA, Velloso LA. Consumption of a fat-rich diet activates a proinflammatory response and induces insulin resistance in the hypothalamus. Endocrinology. 2005 Oct;146(10):4192-9. DOI: 10.1210/en.2004-1520.

217. Wang X, Ge A, Cheng M, Guo F, Zhao M, Zhou X, Liu L, Yang N. Increased hypothalamic inflammation associated with the susceptibility to obesity in rats exposed to high-fat diet. Exp Diabetes Res. 2012 Jan;2012:847246. DOI: 10.1155/2012/847246.

218. Velloso LA, Schwartz MW. Altered hypothalamic function in diet-induced obesity. Int J Obes (Lond). 2011 Dec;35(12):1455-65. DOI: 10.1038/ijo.2011.56.

219. Liu Z, Patil IY, Jiang T, Sancheti H, Walsh JP, Stiles BL, Yin F, Cadenas E. High-fat diet induces hepatic insulin resistance and impairment of synaptic plasticity. PLoS One. 2015 Jan;10(5):e0128274. DOI: 10.1371/journal.pone.0128274.

220. Petrov D, Pedrós I, Artiach G, Sureda FX, Barroso E, Pallàs M, Casadesús G, Beas-Zarate C, Carro E, Ferrer I, Vazquez-Carrera M, Folch J, Camins A. High-fat diet-induced deregulation of hippocampal insulin signaling and mitochondrial homeostasis deficiences contribute to Alzheimer disease pathology in rodents. Biochim Biophys Acta. 2015 Sep;1852(9):1687-99. DOI: 10.1016/j.bbadis.2015.05.004.

221. Magnusson KR, Hauck L, Jeffrey BM, Elias V, Humphrey A, Nath R, Perrone A, Bermudez LE. Relationships between diet-related changes in the gut microbiome and cognitive flexibility. Neuroscience. 2015 Aug 6;300:128-40. DOI: 10.1016/j.neuroscience.2015.05.016.

222. Guri AJ, Hontecillas R, Ferrer G, Casagran O, Wankhade U, Noble AM, Eizirik DL, Ortis F, Cnop M, Liu D, Si H, Bassaganya-Riera J. Loss of PPAR gamma in immune cells impairs the ability of abscisic acid to improve insulin sensitivity by suppressing monocyte chemoattractant protein-1 expression and macrophage infiltration into white adipose tissue. J Nutr Biochem. 2008 Apr;19(4):216-28. DOI: 10.1016/j.jnutbio.2007.02.010.

223. Kamara K, Eskay R, Castonguay T. High-fat diets and stress responsivity. Physiol Behav. 1998 Apr;64(1):1-6.

224. Cruces J, Venero C, Pereda-Pérez I, De la Fuente M. A higher anxiety state in old rats after social isolation is associated to an impairment of the immune response. J Neuroimmunol. 2014 Dec 15;277(1-2):18-25. DOI: 10.1016/j.jneuroim.2014.09.011. 
225. Turner P V, Pekow C, Vasbinder MA, Brabb T. Administration of substances to laboratory animals: equipment considerations, vehicle selection, and solute preparation. J Am Assoc Lab Anim Sci. 2011;50(5):614-27.

226. McNeilly AD, Williamson R, Sutherland C, Balfour DJK, Stewart CA. High fat feeding promotes simultaneous decline in insulin sensitivity and cognitive performance in a delayed matching and non-matching to position task. Behav Brain Res. 2011 Feb 2;217(1):134-41. DOI: 10.1016/j.bbr.2010.10.017.

227. McNay EC, Ong CT, McCrimmon RJ, Cresswell J, Bogan JS, Sherwin RS. Hippocampal memory processes are modulated by insulin and high-fat-induced insulin resistance. Neurobiol Learn Mem. 2010 May;93(4):546-53. DOI: 10.1016/j.nlm.2010.02.002.

228. Valladolid-Acebes I, Stucchi P, Cano V, Fernández-Alfonso MS, Merino B, Gil-Ortega M, Fole A, Morales L, Ruiz-Gayo M, Del Olmo N. High-fat diets impair spatial learning in the radial-arm maze in mice. Neurobiol Learn Mem. 2011 Jan;95(1):80-5. DOI: 10.1016/j.nlm.2010.11.007.

229. Bassaganya-Riera J, Guri AJ, Lu P, Climent M, Carbo A, Sobral BW, Horne WT, Lewis SN, Bevan DR, Hontecillas R. Abscisic acid regulates inflammation via ligand-binding domain-independent activation of peroxisome proliferator-activated receptor $\gamma . \quad J$ Biol Chem. 2011 Jan 28;286(4):2504-16. DOI: 10.1074/jbc.M110.160077.

230. Bassaganya-Riera J, Guri AJ, Hontecillas R. Treatment of Obesity-Related Complications with Novel Classes of Naturally Occurring PPAR Agonists. J Obes. 2011 Jan;2011:897894. DOI: 10.1155/2011/897894.

231. Kariharan T, Nanayakkara G, Parameshwaran K, Bagasrawala I, Ahuja M, Abdel-Rahman E, Amin AT, Dhanasekaran M, Suppiramaniam V, Amin RH. Central activation of PPAR-gamma ameliorates diabetes induced cognitive dysfunction and improves BDNF expression. Neurobiol Aging. 2015 Mar;36(3):1451-61. DOI: 10.1016/j.neurobiolaging.2014.09.028.

232. Nenov MN, Tempia F, Denner L, Dineley KT, Laezza F. Impaired firing properties of dentate granule neurons in an Alzheimer's disease animal model are rescued by PPAR $\gamma$ agonism. J Neurophysiol. 2015 Mar 15;113(6):1712-26. DOI: 10.1152/jn.00419.2014.

233. Olton D, Markowska A, Voytko ML, Givens B, Gorman L, Wenk G. Basal forebrain cholinergic system: a functional analysis. Adv Exp Med Biol. 1991 Jan;295:353-72.

234. DEMBER WN, FOWLER H. Spontaneous alternation behavior. Psychol Bull. 1958 Nov;55(6):412-28.

235. Bodrato N, Franco L, Fresia C, Guida L, Usai C, Salis A, Moreschi I, Ferraris C, Verderio C, Basile G, Bruzzone S, Scarfi S, De Flora A, Zocchi E. Abscisic acid activates the murine microglial cell line N9 through the second messenger cyclic ADP-ribose. J Biol Chem. 2009 May 29;284(22):14777-87. DOI: 10.1074/jbc.M802604200.

236. Jiang SX, Benson CL, Zaharia LI, Abrams SR, Hou ST. Abscisic acid does not evoke calcium influx in murine primary microglia and immortalised murine microglial BV-2 and N9 cells. Biochem Biophys Res Commun. 2010 Oct 22;401(3):435-9. DOI: 10.1016/j.bbrc.2010.09.074.

237. Neumann H, Kotter MR, Franklin RJM. Debris clearance by microglia: an essential link between degeneration and regeneration. Brain. 2009 Feb;132(Pt 2):288-95. DOI: 10.1093/brain/awn109.

238. Smith JA, Das A, Ray SK, Banik NL. Role of pro-inflammatory cytokines released from microglia in neurodegenerative diseases. Brain Res Bull. 2012 Jan 4;87(1):10-20. DOI: 10.1016/j.brainresbull.2011.10.004.

239. Thaler JP, Yi C-X, Schur EA, Guyenet SJ, Hwang BH, Dietrich MO, Zhao X, Sarruf DA, Izgur V, Maravilla KR, Nguyen HT, Fischer JD, Matsen ME, Wisse BE, Morton GJ, Horvath TL, Baskin DG, Tschöp MH, Schwartz MW. Obesity is associated with hypothalamic injury in rodents and humans. J Clin Invest. 2012 Jan;122(1):153-62. DOI: 10.1172/JCI59660.

240. Lozano D, Gonzales-Portillo GS, Acosta S, de la Pena I, Tajiri N, Kaneko Y, Borlongan C V. Neuroinflammatory responses to traumatic brain injury: etiology, clinical consequences, and therapeutic opportunities. Neuropsychiatr Dis Treat. 2015;11:97-106. DOI: 10.2147/NDT.S65815.

241. Madinier A, Bertrand N, Mossiat C, Prigent-Tessier A, Beley A, Marie C, Garnier P. Microglial involvement in neuroplastic changes following focal brain ischemia in rats. PLoS One. 2009 Jan;4(12):e8101. DOI: 10.1371/journal.pone.0008101.

242. Bouvier DS, Murai KK. Synergistic actions of microglia and astrocytes in the progression of Alzheimer's disease. J Alzheimers Dis. 2015 Jan;45(4):1001-14. DOI: 10.3233/JAD-143156.

243. Perry VH, Holmes C. Microglial priming in neurodegenerative disease. Nat Rev Neurol. 2014 Apr;10(4):21724. DOI: $10.1038 /$ nrneurol.2014.38. 
244. Riazi K, Galic M a, Kuzmiski JB, Ho W, Sharkey K a, Pittman QJ. Microglial activation and TNFalpha production mediate altered CNS excitability following peripheral inflammation. Proc Natl Acad Sci U S A. 2008;105(44):17151-6. DOI: 10.1073/pnas.0806682105.

245. Najjar S, Pearlman DM. Neuroinflammation and white matter pathology in schizophrenia: systematic review. Schizophr Res. 2015 Jan;161(1):102-12. DOI: 10.1016/j.schres.2014.04.041.

246. Rial D, Lemos C, Pinheiro H, Duarte JM, Gonçalves FQ, Real JI, Prediger RD, Gonçalves N, Gomes CA, Canas PM, Agostinho P, Cunha RA. Depression as a Glial-Based Synaptic Dysfunction. Front Cell Neurosci. 2015 Jan;9:521. DOI: 10.3389/fncel.2015.00521.

247. McLarnon JG. Microglial chemotactic signaling factors in Alzheimer's disease. Am J Neurodegener Dis. 2012;1(3):199-204.

248. Lazarov O, Hollands C. Hippocampal neurogenesis: Learning to remember. Prog Neurobiol. 2016 Mar;138140:1-18. DOI: 10.1016/j.pneurobio.2015.12.006.

249. Lang BT, Yan Y, Dempsey RJ, Vemuganti R. Impaired neurogenesis in adult type-2 diabetic rats. Brain Res. 2009 Mar;1258:25-33. DOI: 10.1016/j.brainres.2008.12.026.

250. Ryan SM, Nolan YM. Neuroinflammation negatively affects adult hippocampal neurogenesis and cognition: can exercise compensate? Neurosci Biobehav Rev. 2016 Feb;61:121-31. DOI: 10.1016/j.neubiorev.2015.12.004.

251. Ramirez S, Liu X, Lin P-A, Suh J, Pignatelli M, Redondo RL, Ryan TJ, Tonegawa S. Creating a False Memory in the Hippocampus. Science (80- ). 2013;341(6144).

252. O'Leary OF, Cryan JF. A ventral view on antidepressant action: roles for adult hippocampal neurogenesis along the dorsoventral axis. Trends Pharmacol Sci. 2014 Dec;35(12):675-87. DOI: 10.1016/j.tips.2014.09.011.

253. Stephenson J, Nutma E, van der Valk P, Amor S. Inflammation in CNS neurodegenerative diseases. Vol. 154, Immunology. Blackwell Publishing Ltd; 2018. p. 204-19. DOI: 10.1111/imm.12922.

254. Breitner, M.D., M.P.H JCS. THE ROLE OF ANTI-INFLAMMATORY DRUGS IN THE PREVENTION AND TREATMENT OF ALZHEIMER'S DISEASE. Annu Rev Med. 1996 Feb;47(1):401-11. DOI: 10.1146/annurev.med.47.1.401.

255. Eikelenboom P, Bate C, Van Gool WA, Hoozemans JJM, Rozemuller JM, Veerhuis R, Williams A. Neuroinflammation in Alzheimer's disease and prion disease. Vol. 40, GLIA. Glia; 2002. p. 232-9. DOI: 10.1002/glia.10146.

256. Eikelenboom P, Veerhuis R, Scheper W, Rozemuller AJM, Van Gool WA, Hoozemans JJM. The significance of neuroinflammation in understanding Alzheimer's disease. Vol. 113, Journal of Neural Transmission. J Neural Transm (Vienna); 2006. p. 1685-95. DOI: 10.1007/s00702-006-0575-6.

257. Heneka MT, Carson MJ, Khoury J El, Landreth GE, Brosseron F, Feinstein DL, Jacobs AH, Wyss-Coray T, Vitorica J, Ransohoff RM, Herrup K, Frautschy SA, Finsen B, Brown GC, Verkhratsky A, Yamanaka K, Koistinaho J, Latz E, Halle A, Petzold GC, Town T, Morgan D, Shinohara ML, Perry VH, Holmes C, Bazan NG, Brooks DJ, Hunot S, Joseph B, Deigendesch N, Garaschuk O, Boddeke E, Dinarello CA, Breitner JC, Cole GM, Golenbock DT, Kummer MP. Neuroinflammation in Alzheimer's disease. Vol. 14, The Lancet Neurology. Lancet Publishing Group; 2015. p. 388-405. DOI: 10.1016/S1474-4422(15)70016-5.

258. Yuan M, Konstantopoulos N, Lee J, Hansen L, Li ZW, Karin M, Shoelson SE. Reversal of Obesity- and DietInduced Insulin Resistance with Salicylates or Targeted Disruption of Ikkbeta. Science (80- ). 2001 Aug;293(5535):1673-7. DOI: 10.1126/science.1061620.

259. Gao Z, Hwang D, Bataille F, Lefevre M, York D, Quon MJ, Ye J. Serine Phosphorylation of Insulin Receptor Substrate 1 by Inhibitor B Kinase Complex. J Biol Chem. 2002 Dec;277(50):48115-21. DOI: 10.1074/jbc.M209459200.

260. de la Monte SM. Insulin Resistance and Neurodegeneration: Progress Towards the Development of New Therapeutics for Alzheimer's Disease. Vol. 77, Drugs. Springer International Publishing; 2017. p. 47-65. DOI: 10.1007/s40265-016-0674-0.

261. Wang D, Yan J, Chen J, Wu W, Zhu X, Wang Y. Naringin Improves Neuronal Insulin Signaling, Brain Mitochondrial Function, and Cognitive Function in High-Fat Diet-Induced Obese Mice. Cell Mol Neurobiol. 2015;35(7):1061-71. DOI: 10.1007/s10571-015-0201-y.

262. Pipatpiboon N, Pratchayasakul W, Chattipakorn N, Chattipakorn SC. PPAR agonist improves neuronal insulin receptor function in hippocampus and brain mitochondria function in rats with insulin resistance induced by long term high-fat diets. Endocrinology. 2012;153(1):329-38. DOI: 10.1210/en.2011-1502. 
263. Sun X, Yao H, Douglas RM, Gu XQ, Wang J, Haddad GG. Insulin/PI3K signaling protects dentate neurons from oxygenâ€"glucose deprivation in organotypic slice cultures. J Neurochem. 2010 Jan;112(2):377-88. DOI: 10.1111/j.1471-4159.2009.06450.x.

264. Gage FH, van Praag H, Kempermann G. Running increases cell proliferation and neurogenesis in the adult mousedentate gyrus. Nat Neurosci. 1999 Mar;2(3):266-70. DOI: 10.1038/6368.

265. Vivar C, Potter MC, van Praag H. All about running: synaptic plasticity, growth factors and adult hippocampal neurogenesis. Curr Top Behav Neurosci. 2013;15:189-210. DOI: 10.1007/7854_2012_220.

266. Timinkul A, Kato M, Omori T, Deocaris CC, Ito A, Kizuka T, Sakairi Y, Nishijima T, Asada T, Soya H. Enhancing effect of cerebral blood volume by mild exercise in healthy young men: A near-infrared spectroscopy study. Neurosci Res. 2008 Jul;61(3):242-8. DOI: 10.1016/j.neures.2008.03.012.

267. Nishijima T, Piriz J, Duflot S, Fernandez AM, Gaitan G, Gomez-Pinedo U, Verdugo JMG, Leroy F, Soya H, $\mathrm{Nu}$ ?ez A, Torres-Aleman I. Neuronal Activity Drives Localized Blood-Brain-Barrier Transport of Serum Insulin-like Growth Factor-I into the CNS. Neuron. 2010 Sep;67(5):834-46. DOI: 10.1016/j.neuron.2010.08.007.

268. Choi Y-S, Cho H-Y, Hoyt KR, Naegele JR, Obrietan K. IGF-1 receptor-mediated ERK/MAPK signaling couples status epilepticus to progenitor cell proliferation in the subgranular layer of the dentate gyrus. Glia. 2008 May;56(7):791-800. DOI: 10.1002/glia.20653.

269. Wei Z, Liao J, Qi F, Meng Z, Pan S. Evidence for the contribution of BDNF-TrkB signal strength in neurogenesis: An organotypic study. Neurosci Lett. 2015 Oct;606:48-52. DOI: 10.1016/j.neulet.2015.08.032.

270. Wrann CD, White JP, Salogiannnis J, Laznik-Bogoslavski D, Wu J, Ma D, Lin JD, Greenberg ME, Spiegelman BM. Exercise Induces Hippocampal BDNF through a PGC-1 $\alpha /$ FNDC5 Pathway. Cell Metab. 2013 Nov;18(5):649-59. DOI: 10.1016/j.cmet.2013.09.008.

271. Ding Q, Vaynman S, Akhavan M, Ying Z, Gomez-Pinilla F. Insulin-like growth factor I interfaces with brainderived neurotrophic factor-mediated synaptic plasticity to modulate aspects of exercise-induced cognitive function. Neuroscience. $2006 \mathrm{Jan} ; 140(3): 823-33$. DOI: 10.1016/j.neuroscience.2006.02.084.

272. Bedse G, Di Domenico F, Serviddio G, Cassano T. Aberrant insulin signaling in Alzheimer's disease: Current knowledge. Front Neurosci. 2015;9(MAY):1-13. DOI: 10.3389/fnins.2015.00204.

273. Wilcox G. Insulin and insulin resistance. Clin Biochem Rev. 2005;26(2):19-39. DOI: 10.1016/S00257125(03)00128-7.

274. Duarte AI, Moreira PI, Oliveira CR. Insulin in central nervous system: More than just a peripheral hormone. $J$ Aging Res. 2012;2012. DOI: 10.1155/2012/384017.

275. Zemva J, Udelhoven M, Moll L, Freude S, Stöhr O, Brönneke HS, Drake RB, Krone W, Schubert M. Neuronal overexpression of insulin receptor substrate 2 leads to increased fat mass, insulin resistance, and glucose intolerance during aging. Age (Dordr). 2013 Oct;35(5):1881-97. DOI: 10.1007/s11357-012-9491-x.

276. Zhao W, Chen H, Xu H, Moore E, Meiri N, Quon MJ, Alkon DL. Brain insulin receptors and spatial memory. Correlated changes in gene expression, tyrosine phosphorylation, and signaling molecules in the hippocampus of water maze trained rats. J Biol Chem. 1999 Dec;274(49):34893-902. DOI: 10.1074/JBC.274.49.34893.

277. Mir S, Cai W, Carlson SW, Saatman KE, Andres DA. IGF-1 mediated Neurogenesis Involves a Novel RIT1/Akt/Sox2 Cascade. Sci Rep. 2017 Jun;7(1):3283. DOI: 10.1038/s41598-017-03641-9.

278. Nieto-Estévez V, Defterali Ç, Vicario-Abejón C. IGF-I: A Key Growth Factor that Regulates Neurogenesis and Synaptogenesis from Embryonic to Adult Stages of the Brain. Front Neurosci. 2016;10:52. DOI: 10.3389/fnins.2016.00052.

279. MARKS JL, PORTE D, STAHL WL, BASKIN DG. Localization of insulin receptor mRNA in rat brain by in situ hybridization. Endocrinology. 1990 Dec;127(6):3234-6. DOI: 10.1210/endo-127-6-3234.

280. Schmitz-Peiffer C. Signalling aspects of insulin resistance in skeletal muscle: mechanisms induced by lipid oversupply. Cell Signal. 2000 Oct;12(9-10):583-94.

281. Potashnik R, Bloch-Damti A, Bashan N, Rudich A. IRS1 degradation and increased serine phosphorylation cannot predict the degree of metabolic insulin resistance induced by oxidative stress. Diabetologia. 2003 May;46(5):639-48. DOI: 10.1007/s00125-003-1097-5.

282. Cardoso S, Santos R, Correia S, Carvalho C, Zhu X, Lee H-G, Casadesus G, Smith MA, Perry G, Moreira PI. Insulin and Insulin-Sensitizing Drugs in Neurodegeneration: Mitochondria as Therapeutic Targets. Pharmaceuticals. 2009 Dec;2(3):250-86. DOI: 10.3390/ph2030250. 
283. Lu P, Hontecillas R, Horne WT, Carbo A, Viladomiu M, Pedragosa M, Bevan DR, Lewis SN, Bassaganya-Riera J. Computational modeling-based discovery of novel classes of anti-inflammatory drugs that target lanthionine synthetase C-like protein 2. PLoS One. 2012;7(4). DOI: 10.1371/journal.pone.0034643.

284. Paxinos G, Watson C. The Rat Brain in Stereotaxic Coordinates : Hard Cover Edition. Elsevier Science; 2013. $466 \mathrm{p}$.

285. Pearson K. VII. Note on regression and inheritance in the case of two parents. Proc R Soc London. 1895 Dec 31;58(347-352):240-2. DOI: 10.1098/rspl.1895.0041.

286. Stigler SM. Francis Galton's account of the invention of correlation. Stat Sci. 1989;4(2):73-9. DOI: $10.1214 / \mathrm{ss} / 1177012580$.

287. Galton F. Regression Towards Mediocrity in Hereditary Stature. J Anthropol Inst Gt Britain Irel. 1886;15:246. DOI: $10.2307 / 2841583$.

288. Cohen J. Statistical Power Analysis for the Behavioral Sciences Second Edition. Erlbaum Associates L, editor. Hillsdale; 1988.

289. Elmarakby AA, Imig JD. Obesity is the major contributor to vascular dysfunction and inflammation in high-fat diet hypertensive rats. Clin Sci. 2010 Feb;118(4):291-301. DOI: 10.1042/CS20090395.

290. Bhat NR, Thirumangalakudi L. Increased tau phosphorylation and impaired brain insulin/IGF signaling in mice fed a high fat/high cholesterol diet. J Alzheimers Dis. 2013;36(4):781-9. DOI: 10.3233/JAD-2012-121030.

291. Korzhevskii DE, Karpenko MN, Kirik O V. Microtubule-Associated Proteins as Indicators of Differentiation and the Functional State of Nerve Cells. Neurosci Behav Physiol. 2012 Mar;42(3):215-22. DOI: $10.1007 / \mathrm{s} 11055-012-9556-4$.

292. Alfaro FJ, Gavrieli A, Saade-Lemus P, Lioutas V-A, Upadhyay J, Novak V. White matter microstructure and cognitive decline in metabolic syndrome: a review of diffusion tensor imaging. Metabolism. 2018 Jan;78:52-68. DOI: 10.1016/j.metabol.2017.08.009.

293. Stump M, Mukohda M, Hu C, Sigmund CD. PPAR $\gamma$ Regulation in Hypertension and Metabolic Syndrome. Curr Hypertens Rep. 2015 Dec;17(12):89. DOI: 10.1007/s11906-015-0601-x.

294. Ullah F, Liang A, Rangel A, Gyengesi E, Niedermayer G, Münch G. High bioavailability curcumin: an antiinflammatory and neurosupportive bioactive nutrient for neurodegenerative diseases characterized by chronic neuroinflammation. Vol. 91, Archives of Toxicology. Springer Verlag; 2017. p. 1623-34. DOI: 10.1007/s00204017-1939-4.

295. Lin J, Chen A. Activation of peroxisome proliferator-activated receptor- $\gamma$ by curcumin blocks the signaling pathways for PDGF and EGF in hepatic stellate cells. Lab Investig. 2008 May;88(5):529-40. DOI: 10.1038/labinvest.2008.20.

296. Bernardo A, Minghetti L. PPAR-gamma agonists as regulators of microglial activation and brain inflammation. Curr Pharm Des. 2006;12(1):93-109.

297. Grote CW, Morris JK, Ryals JM, Geiger PC, Wright DE. Insulin Receptor Substrate 2 Expression and Involvement in Neuronal Insulin Resistance in Diabetic Neuropathy. Exp Diabetes Res. 2011;2011:1-12. DOI: $10.1155 / 2011 / 212571$.

298. Agarwal P, Srivastava R, Srivastava AK, Ali S, Datta M. miR-135a targets IRS2 and regulates insulin signaling and glucose uptake in the diabetic gastrocnemius skeletal muscle. Biochim Biophys Acta - Mol Basis Dis. 2013 Aug;1832(8):1294-303. DOI: 10.1016/j.bbadis.2013.03.021.

299. Feng H-L, Li R-S, Wang H, Ren Y, Sun H-Y, Yang J-D, Wang P-W. [Effect of curcumin on hippocampal IRS-1 and p-IRS-1 expressions in APP/PS1 double transgenic mice]. Zhongguo Zhong Yao Za Zhi. 2013 May;38(9):1290-4.

300. Arkan MC, Hevener AL, Greten FR, Maeda S, Li Z-W, Long JM, Wynshaw-Boris A, Poli G, Olefsky J, Karin M. IKK- $\beta$ links inflammation to obesity-induced insulin resistance. Nat Med. 2005 Feb;11(2):191-8. DOI: $10.1038 / \mathrm{nm} 1185$.

301. Hacker H, Karin M. Regulation and Function of IKK and IKK-Related Kinases. Sci STKE. 2006 Oct;2006(357):re13-re13. DOI: 10.1126/stke.3572006re13.

302. Zhang Y, Huang N, Yan F, Jin H, Zhou S, Shi J, Jin F. Diabetes mellitus and Alzheimer's disease: GSK-3 $\beta$ as a potential link. Behav Brain Res. 2018 Feb;339:57-65. DOI: 10.1016/J.BBR.2017.11.015.

303. Castorena CM, Arias EB, Sharma N, Cartee GD. Effects of a brief high-fat diet and acute exercise on the 
mTORC1 and IKK/NF-kB pathways in rat skeletal muscle. Appl Physiol Nutr Metab. 2015 Mar;40(3):251-62. DOI: 10.1139/apnm-2014-0412.

304. Poulose SM, Miller MG, Scott T, Shukitt-Hale B. Nutritional Factors Affecting Adult Neurogenesis and Cognitive Function. Adv Nutr. 2017 Nov;8(6):804-11. DOI: 10.3945/an.117.016261.

305. Yoo DY, Kim W, Nam SM, Yoo K-Y, Lee CH, Choi JH, Won M-H, Hwang IK, Yoon YS. Reduced Cell Proliferation and Neuroblast Differentiation in the Dentate Gyrus of High Fat Diet-Fed Mice are Ameliorated by Metformin and Glimepiride Treatment. Neurochem Res. 2011 Dec;36(12):2401-8. DOI: 10.1007/s11064-0110566-3.

306. Bekinschtein P, Cammarota M, Medina JH. BDNF and memory processing. Neuropharmacology. 2014 Jan;76:677-83. DOI: 10.1016/j.neuropharm.2013.04.024.

307. Wang H, Wang B, Yin H, Zhang G, Yu L, Kong X, Yuan H, Fang X, Liu Q, Liu C, Shi L. Reduced neurotrophic factor level is the early event before the functional neuronal deficiency in high-fat diet induced obese mice. Metab Brain Dis. 2017 Feb;32(1):247-57. DOI: 10.1007/s11011-016-9905-z.

308. Stranahan AM, Norman ED, Lee K, Cutler RG, Telljohann RS, Egan JM, Mattson MP. Diet-induced insulin resistance impairs hippocampal synaptic plasticity and cognition in middle-aged rats. Hippocampus. 2008 Nov;18(11):1085-8. DOI: 10.1002/hipo.20470.

309. Mi Y, Qi G, Fan R, Qiao Q, Sun Y, Gao Y, Liu X. EGCG ameliorates high-fat- and high-fructose-induced cognitive defects by regulating the IRS/AKT and ERK/CREB/BDNF signaling pathways in the CNS. FASEB J. 2017 Nov;31(11):4998-5011. DOI: 10.1096/fj.201700400RR.

310. Carey AN, Gildawie KR, Rovnak A, Thangthaeng N, Fisher DR, Shukitt-Hale B. Blueberry supplementation attenuates microglia activation and increases neuroplasticity in mice consuming a high-fat diet. Nutr Neurosci. 2017 Sep;1-11. DOI: 10.1080/1028415X.2017.1376472.

311. Yamada M, Ohnishi H, Sano S i, Nakatani A, Ikeuchi T, Hatanaka H. Insulin receptor substrate (IRS)-1 and IRS-2 are tyrosine-phosphorylated and associated with phosphatidylinositol 3-kinase in response to brainderived neurotrophic factor in cultured cerebral cortical neurons. J Biol Chem. 1997 Nov;272(48):30334-9.

312. Grimble RF. Inflammatory status and insulin resistance. Curr Opin Clin Nutr Metab Care. 2002 Sep;5(5):551-9.

313. Guillemot-Legris O, Muccioli GG. Obesity-Induced Neuroinflammation: Beyond the Hypothalamus. Trends Neurosci. 2017 Apr;40(4):237-53. DOI: 10.1016/j.tins.2017.02.005.

314. Wadhwa M, Prabhakar A, Ray K, Roy K, Kumari P, Jha PK, Kishore K, Kumar S, Panjwani U. Inhibiting the microglia activation improves the spatial memory and adult neurogenesis in rat hippocampus during $48 \mathrm{~h}$ of sleep deprivation. J Neuroinflammation. 2017 Nov;14(1):222. DOI: 10.1186/s12974-017-0998-z.

315. Crews L, Rockenstein E, Masliah E. APP transgenic modeling of Alzheimer's disease: mechanisms of neurodegeneration and aberrant neurogenesis. Brain Struct Funct. 2010 Mar;214(2-3):111-26. DOI: 10.1007/s00429-009-0232-6.

316. Nuzzo D, Picone P, Baldassano S, Caruana L, Messina E, Marino Gammazza A, Cappello F, Mulè F, Di Carlo M. Insulin Resistance as Common Molecular Denominator Linking Obesity to Alzheimer's Disease. Curr Alzheimer Res. 2015;12(8):723-35.

317. Choi J, Jang J, Son D, Im H-S, Kim J, Park J, Choi W, Han S-B, Hong J. Antarctic Krill Oil Diet Protects against Lipopolysaccharide-Induced Oxidative Stress, Neuroinflammation and Cognitive Impairment. Int J Mol Sci. 2017 Nov;18(12):2554. DOI: 10.3390/ijms18122554.

318. Al-Delaimy WK, von Muhlen D, Barrett-Connor E. Insulinlike growth factor-1, insulinlike growth factor binding protein-1, and cognitive function in older men and women. J Am Geriatr Soc. 2009 Aug;57(8):1441-6. DOI: 10.1111/j.1532-5415.2009.02343.x.

319. Cholerton B, Baker LD, Craft S. Insulin, cognition, and dementia. Eur J Pharmacol. 2013 Nov 5;719(1-3):1709. DOI: 10.1016/j.ejphar.2013.08.008.

320. Long YC, Cheng Z, Copps KD, White MF. Insulin receptor substrates Irs1 and Irs2 coordinate skeletal muscle growth and metabolism via the Akt and AMPK pathways. Mol Cell Biol. 2011 Feb;31(3):430-41. DOI: 10.1128/MCB.00983-10.

321. Shirakami A, Toyonaga T, Tsuruzoe K, Shirotani T, Matsumoto K, Yoshizato K, Kawashima J, Hirashima Y, Miyamura N, Kahn CR, Araki E. Heterozygous knockout of the IRS-1 gene in mice enhances obesity-linked insulin resistance: a possible model for the development of type 2 diabetes. J Endocrinol. 2002 Aug;174(2):309_ 19. 
322. Dong X, Park S, Lin X, Copps K, Yi X, White MF. Irs1 and Irs2 signaling is essential for hepatic glucose homeostasis and systemic growth. J Clin Invest. 2006 Jan;116(1):101-14. DOI: 10.1172/JCI25735.

323. Guo S, Copps KD, Dong X, Park S, Cheng Z, Pocai A, Rossetti L, Sajan M, Farese R V, White MF. The Irs1 branch of the insulin signaling cascade plays a dominant role in hepatic nutrient homeostasis. Mol Cell Biol. 2009 Sep;29(18):5070-83. DOI: 10.1128/MCB.00138-09.

324. Costello D a., Claret M, Al-Qassab H, Plattner F, Irvine EE, Choudhury AI, Giese KP, Withers DJ, Pedarzani P. Brain deletion of insulin receptor substrate 2 disrupts hippocampal synaptic plasticity and metaplasticity. PLoS One. 2012;7(2):30-4. DOI: 10.1371/journal.pone.0031124.

325. Freude S, Hettich MM, Schumann C, Stöhr O, Koch L, Köhler C, Udelhoven M, Leeser U, Müller M, Kubota N, Kadowaki T, Krone W, Schröder H, Brüning JC, Schubert M. Neuronal IGF-1 resistance reduces A $\beta$ accumulation and protects against premature death in a model of Alzheimer's disease. FASEB J. 2009 Oct;23(10):3315-24. DOI: 10.1096/fj.09-132043.

326. Sadagurski M, Cheng Z, Rozzo A, Palazzolo I, Kelley GR, Dong X, Krainc D, White MF. IRS2 increases mitochondrial dysfunction and oxidative stress in a mouse model of Huntington disease. J Clin Invest. 2011 Oct 3;121(10):4070-81. DOI: 10.1172/JCI46305.

327. Rondinone CM, Wang LM, Lonnroth P, Wesslau C, Pierce JH, Smith U. Insulin receptor substrate (IRS) 1 is reduced and IRS-2 is the main docking protein for phosphatidylinositol 3-kinase in adipocytes from subjects with non-insulin-dependent diabetes mellitus. Proc Natl Acad Sci U S A. 1997 Apr;94(8):4171-5. DOI: 10.1073/pnas.94.8.4171.

328. Yamada M, Ohnishi H, Sano SI, Nakatani A, Ikeuchi T, Hatanaka H. Insulin receptor substrate (IRS)-1 and IRS2 are tyrosine- phosphorylated and associated with phosphatidylinositol 3-kinase in response to brain-derived neurotrophic factor in cultured cerebral cortical neurons. J Biol Chem. 1997 Nov 28;272(48):30334-9. DOI: $10.1074 / j b c .272 .48 .30334$.

329. Hanke S, Mann M. The phosphotyrosine interactome of the insulin receptor family and its substrates IRS-1 and IRS-2. Mol Cell Proteomics. 2009 Mar;8(3):519-34. DOI: 10.1074/mcp.M800407-MCP200.

330. Whelan S a., Dias WB, Thiruneelakantapillai L, Daniel Lane M, Hart GW. Regulation of insulin receptor substrate 1 (IRS-1)/AKT kinase-mediated insulin signaling by O-linked ??-N-acetylglucosamine in 3T3-L1 adipocytes. J Biol Chem. 2010;285(8):5204-11. DOI: 10.1074/jbc.M109.077818.

331. Costa MM, Violato NM, Taboga SR, Góes RM, Bosqueiro JR. Reduction of insulin signalling pathway IRS1/IRS-2/AKT/mTOR and decrease of epithelial cell proliferation in the prostate of glucocorticoid-treated rats. Int J Exp Pathol. 2012 Jun;93(3):188-95. DOI: 10.1111/j.1365-2613.2012.00817.x.

332. Hemmings BA, Restuccia DF. PI3K-PKB/Akt pathway. Cold Spring Harb Perspect Biol. 2012 Sep;4(9):a011189. DOI: 10.1101/cshperspect.a011189.

333. Machado-Neto JA, Fenerich BA, Alves APNR, Fernandes JC, Scopim-Ribeiro R, Coelho-Silva JL, Traina F. Insulin Substrate Receptor (IRS) proteins in normal and malignant hematopoiesis. Clinics. 2018;73(Suppl 1). DOI: 10.6061/CLINICS/2018/E566S.

334. Copps KD, White MF. Regulation of insulin sensitivity by serine/threonine phosphorylation of insulin receptor substrate proteins IRS1 and IRS2. Diabetologia. 2012 Oct;55(10):2565-82. DOI: 10.1007/s00125-012-2644-8.

335. Moloney AM, Griffin RJ, Timmons S, O’Connor R, Ravid R, O’Neill C. Defects in IGF-1 receptor, insulin receptor and IRS-1/2 in Alzheimer's disease indicate possible resistance to IGF-1 and insulin signalling. Neurobiol Aging. 2010 Feb;31(2):224-43. DOI: 10.1016/j.neurobiolaging.2008.04.002.

336. Dineley KT, Jahrling JB, Denner L. Insulin resistance in Alzheimer's disease. Neurobiol Dis. 2014 Dec;72 Pt A:92-103. DOI: 10.1016/j.nbd.2014.09.001.

337. Du G, Yonekubo J, Zeng Y, Osisami M, Frohman MA. Design of expression vectors for RNA interference based on miRNAs and RNA splicing. FEBS J. 2006 Dec;273(23):5421-7. DOI: 10.1111/j.17424658.2006.05534.x.

338. Addgene: AAV Purification [Internet].

339. Yankelevitch-Yahav R, Franko M, Huly A, Doron R. The Forced Swim Test as a Model of Depressive-like Behavior. J Vis Exp. 2015 Mar 2;(97). DOI: 10.3791/52587.

340. Neves FS, Marques PT, Barros-Aragão F, Nunes NB, Venancio AM, Cozachenco D, Frozza RL, Passos GF, Costa R, de Oliveira J, Engel DF, De Bem AF, Benjamim CF, De Felice FG, Ferreira ST, Clarke JR, Figueiredo CP. Brain-defective insulin signaling is associated to late cognitive impairment in post-septic mice. Mol 
Neurobiol. 2018;55(1):435-44. DOI: 10.1007/s12035-016-0307-3.

341. Spinelli M, Fusco S, Mainardi M, Scala F, Natale F, Lapenta R, Mattera A, Rinaudo M, Li Puma DD, Ripoli C, Grassi A, D'Ascenzo M, Grassi C. Brain insulin resistance impairs hippocampal synaptic plasticity and memory by increasing GluA1 palmitoylation through. Nat Commun. 2017 Dec 1;8(1). DOI: 10.1038/s41467-017-022219.

342. Spinelli M, Fusco S, Grassi C. Brain insulin resistance and hippocampal plasticity: Mechanisms and biomarkers of cognitive decline. Vol. 10, Frontiers in Neuroscience. Frontiers Media S.A.; 2019. DOI: 10.3389/fnins.2019.00788.

343. Golimstok A, Cámpora N, Rojas JI, Fernandez MC, Elizondo C, Soriano E, Cristiano E. Cardiovascular risk factors and frontotemporal dementia: A case-control study. Transl Neurodegener. 2014 Jun 21;3(1). DOI: $10.1186 / 2047-9158-3-13$.

344. Folch J, Olloquequi J, Ettcheto M, Busquets O, Sánchez-López E, Cano A, Espinosa-Jiménez T, García ML, Beas-Zarate C, Casadesús G, Bulló M, Auladell C, Camins A. The Involvement of Peripheral and Brain Insulin Resistance in Late Onset Alzheimer's Dementia. Vol. 11, Frontiers in Aging Neuroscience. Frontiers Media S.A.; 2019. DOI: 10.3389/fnagi.2019.00236.

345. Cao Q, Tan C-C, Xu W, Hu H, Cao X-P, Dong Q, Tan L, Yu J-T. The Prevalence of Dementia: A Systematic Review and Meta-Analysis. J Alzheimer's Dis. 2019 Dec 27;73(3):1-10. DOI: 10.3233/jad-191092.

346. Yan Y, Dominguez S, Fisher DW, Dong H. Sex differences in chronic stress responses and Alzheimer's disease. Vol. 8, Neurobiology of Stress. Elsevier Inc; 2018. p. 120-6. DOI: 10.1016/j.ynstr.2018.03.002.

347. Hebert LE, Scherr PA, McCann JJ, Beckett LA, Evans DA. Is the risk of developing Alzheimer's disease greater for women than for men? Am J Epidemiol. 2001 Jan 15;153(2):132-6. DOI: 10.1093/aje/153.2.132.

348. Miyawaki CE. Association of social isolation and health across different racial and ethnic groups of older Americans. Ageing Soc. 2015 Nov;35(10):2201-28. DOI: 10.1017/S0144686X14000890.

349. Beam CR, Kaneshiro C, Jang JY, Reynolds CA, Pedersen NL, Gatz M. Differences between Women and Men in Incidence Rates of Dementia and Alzheimer's Disease. J Alzheimer's Dis. 2018;64(4):1077-83. DOI: 10.3233/JAD-180141.

350. Payami H, Montee KR, Kaye JA, Bird TD, Yu CE, Wijsman EM, Schellenberg GD. Alzheimer's disease, apolipoprotein E4, and gender. JAMA. 1994 May 4;271(17):1316-7.

351. Ungar L, Altmann A, Greicius MD. Apolipoprotein E, gender, and Alzheimer's disease: an overlooked, but potent and promising interaction. Brain Imaging Behav. 2014 Jun;8(2):262-73. DOI: 10.1007/s11682-013-9272$\mathrm{x}$.

352. Uddin MS, Kabir MT, Al Mamun A, Abdel-Daim MM, Barreto GE, Ashraf GM. APOE and Alzheimer's Disease: Evidence Mounts that Targeting APOE4 may Combat Alzheimer's Pathogenesis. Mol Neurobiol. 2019 Apr;56(4):2450-65. DOI: 10.1007/s12035-018-1237-z.

353. Marcuzzi A, Loganes C, Valencic E, Piscianz E, Monasta L, Bilel S, Bortul R, Celeghini C, Zweyer M, Tommasini A. Neuronal dysfunction associated with cholesterol deregulation. Int J Mol Sci. 2018 May 19;19(5). DOI: $10.3390 /$ ijms 19051523.

354. Jeong W, Lee H, Cho S, Seo J. ApoE4-Induced Cholesterol Dysregulation and Its Brain Cell Type-Specific Implications in the Pathogenesis of Alzheimer's Disease. Mol Cells. 2019 Nov 12; DOI: 10.14348/molcells.2019.0200.

355. Grillo CA, Piroli GG, Lawrence RC, Wrighten SA, Green AJ, Wilson SP, Sakai RR, Kelly SJ, Wilson MA, Mott DD, Reagan LP. Hippocampal insulin resistance impairs spatial learning and synaptic plasticity. Diabetes. 2015 Nov 1;64(11):3927-36. DOI: 10.2337/db15-0596.

356. Degroot A, Treit D. Dorsal and ventral hippocampal cholinergic systems modulate anxiety in the plus-maze and shock-probe tests. Brain Res. 2002 Sep 13;949(1-2):60-70. DOI: 10.1016/S0006-8993(02)02965-7.

357. Degroot A, Treit D. Septal gabaergic and hippocampal cholinergic systems interact in the modulation of anxiety. Neuroscience. 2003 Mar 21;117(2):493-501. DOI: 10.1016/S0306-4522(02)00651-6.

358. Gage FH, Björklund A. Cholinergic septal grafts into the hippocampal formation improve spatial learning and memory in aged rats by an atropine-sensitive mechanism. $J$ Neurosci. 1986 Oct;6(10):2837-47.

359. Ujfalussy B, Kiss T, Orbán G, Hoffmann WE, Erdi P, Hajós M. Pharmacological and computational analysis of alpha-subunit preferential $\mathrm{GABA}(\mathrm{A})$ positive allosteric modulators on the rat septo-hippocampal activity. Neuropharmacology. 2007 Mar;52(3):733-43. DOI: 10.1016/j.neuropharm.2006.09.022. 
360. Jinno S. Structural organization of long-range GABAergic projection system of the hippocampus. Front Neuroanat. 2009;3:13. DOI: 10.3389/neuro.05.013.2009.

361. Eichenbaum H. Thinking about brain cell assemblies. Vol. 261, Science. 1993. p. 993-4. DOI: $10.1126 /$ science. 8351525 .

362. Milenkovic I, Vasiljevic M, Maurer D, Höger H, Klausberger T, Sieghart W. The parvalbumin-positive interneurons in the mouse dentate gyrus express GABAA receptor subunits $\alpha 1, \beta 2$, and $\delta$ along their extrasynaptic cell membrane. Neuroscience. 2013 Dec 19;254:80-96. DOI: 10.1016/j.neuroscience.2013.09.019.

363. Burgess N, Maguire EA, O’Keefe J. The human hippocampus and spatial and episodic memory. Vol. 35, Neuron. Cell Press; 2002. p. 625-41. DOI: 10.1016/S0896-6273(02)00830-9.

364. Leutgeb S, Leutgeb JK, Barnes CA, Moser EI, McNaughton BL, Moser MB. Neuroscience: Independent codes for spatial and episodic memory in hippocampal neuronal ensembles. Science (80- ). 2005 Jul 22;309(5734):619-23. DOI: 10.1126/science.1114037.

365. Yuki D, Sugiura Y, Zaima N, Akatsu H, Takei S, Yao I, Maesako M, Kinoshita A, Yamamoto T, Kon R, Sugiyama K, Setou M. DHA-PC and PSD-95 decrease after loss of synaptophysin and before neuronal loss in patients with Alzheimer's disease. Sci Rep. 2014;4. DOI: 10.1038/srep07130.

366. Grillo CA, Piroli GG, Hendry RM, Reagan LP. Insulin-stimulated translocation of GLUT4 to the plasma membrane in rat hippocampus is PI3-kinase dependent. Brain Res. 2009 Oct 16;1296:35-45. DOI: 10.1016/j.brainres.2009.08.005.

367. Ashrafi G, Wu Z, Farrell RJ, Ryan TA. GLUT4 Mobilization Supports Energetic Demands of Active Synapses. Neuron. 2017 Feb 8;93(3):606-615.e3. DOI: 10.1016/j.neuron.2016.12.020.

368. Li F, Liu K, Wang A, Harris PWR, Vickers MH, Guan J. Cyclic glycine-proline administration normalizes highfat diet-induced synaptophysin expression in obese rats. Neuropeptides. 2019 Aug 1;76. DOI: 10.1016/j.npep.2019.05.006.

369. Tartaglia N, Du J, Tyler WJ, Neale E, Pozzo-Miller L, Lu B. Protein Synthesis-dependent and -independent Regulation of Hippocampal Synapses by Brain-derived Neurotrophic Factor. J Biol Chem. 2001 Oct 5;276(40):37585-93. DOI: 10.1074/jbc.M101683200.

370. Zhang Y, Qiu B, Wang J, Yao Y, Wang C, Liu J. Effects of BDNF-Transfected BMSCs on Neural Functional Recovery and Synaptophysin Expression in Rats with Cerebral Infarction. Mol Neurobiol. 2017 Jul 1;54(5):3813-24. DOI: 10.1007/s12035-016-9946-7.

371. Milenkovic I, Vasiljevic M, Maurer D, Höger H, Klausberger T, Sieghart W. The parvalbumin-positive interneurons in the mouse dentate gyrus express GABAA receptor subunits alpha1, beta2, and delta along their extrasynaptic cell membrane. Neuroscience. 2013 Dec 19;254:80-96. DOI: 10.1016/j.neuroscience.2013.09.019.

372. Zou D, Chen L, Deng D, Jiang D, Dong F, McSweeney C, Zhou Y, Liu L, Chen G, Wu Y, Mao Y. DREADD in Parvalbumin Interneurons of the Dentate Gyrus Modulates Anxiety, Social Interaction and Memory Extinction. Curr Mol Med. 2016 Jan 6;16(1):91-102. DOI: 10.2174/1566524016666151222150024.

373. Escobar I, Xu J, Jackson CW, Perez-Pinzon MA. Altered Neural Networks in the Papez Circuit: Implications for Cognitive Dysfunction after Cerebral Ischemia. J Alzheimers Dis. 2019;67(2):425-46. DOI: 10.3233/JAD180875 .

374. Gaffan EA, Bannerman DM, Warburton EC, Aggleton JP. Rats' processing of visual scenes: effects of lesions to fornix, anterior thalamus, mamillary nuclei or the retrohippocampal region. Behav Brain Res. 2001 Jun;121(12):103-17. DOI: 10.1016/s0166-4328(00)00389-2.

375. Gaffan D, Gaffan EA. Amnesia in man following transection of the fornix: A review. Brain. 1991 Dec;114(6):2611-8. DOI: 10.1093/brain/114.6.2611.

376. Zarei M, Patenaude B, Damoiseaux J, Morgese C, Smith S, Matthews PM, Barkhof F, Rombouts SARB, SanzArigita E, Jenkinson M. Combining shape and connectivity analysis: an MRI study of thalamic degeneration in Alzheimer's disease. Neuroimage. 2010 Jan 1;49(1):1-8. DOI: 10.1016/j.neuroimage.2009.09.001.

377. Vann SD, Erichsen JT, O'Mara SM, Aggleton JP. Selective disconnection of the hippocampal formation projections to the mammillary bodies produces only mild deficits on spatial memory tasks: implications for fornix function. Hippocampus. 2011 Sep;21(9):945-57. DOI: 10.1002/hipo.20796.

378. Vann SD, Nelson AJD. The mammillary bodies and memory: More than a hippocampal relay. In: Progress in Brain Research. Elsevier B.V.; 2015. p. 163-85. DOI: 10.1016/bs.pbr.2015.03.006.

379. Vann SD. Re-evaluating the role of the mammillary bodies in memory. Neuropsychologia. 2010 Jul;48(8):2316- 
27. DOI: 10.1016/j.neuropsychologia.2009.10.019.

380. Faraone S V, Sergeant J, Gillberg C, Biederman J. The worldwide prevalence of ADHD: is it an American condition? World Psychiatry. 2003 Jun;2(2):104-13.

381. American Psychiatric Association. Diagnostic and Statistical Manual of Mental Disorders [Internet]. American Psychiatric Association; 2013. DOI: 10.1176/appi.books.9780890425596.

382. Castellanos FX, Tannock R. Neuroscience of attention-deficit/hyperactivity disorder: The search for endophenotypes. Vol. 3, Nature Reviews Neuroscience. European Association for Cardio-Thoracic Surgery; 2002. p. 617-28. DOI: 10.1038/nrn896.

383. Sánchez-Pérez AM, García-Avilés Á, Gascó HA, Sanjuán J, Olucha-Bordonau FE. Effects of methylphenidate on anxiety. Rev Neurol. 2012;55(8):499-506. DOI: 10.33588/rn.5508.2012355.

384. Zaami S, Tagliabracci A, Berretta P, Busardò FP, Marinelli E. Use of Methylphenidate Analogues as Cognitive Enhancers: The Prelude to Cosmetic Neurology and an Ethical Issue. Front Psychiatry. 2020 Jan 23;10. DOI: 10.3389/fpsyt.2019.01006.

385. Krinzinger H, L Hall C, Groom MJ, T Ansari M, Banaschewski T, Buitelaar JK, Carucci S, Coghill D, Danckaerts M, Dittmann RW, Falissard B, Garas P, Inglis SK, Kovshoff H, Kochhar P, McCarthy S, Nagy P, Neubert A, Roberts S, Sayal K, Sonuga-Barke E, Wong ICK, Xia J, Zuddas A, Hollis C, Konrad K, Liddle EB, ADDUCE Consortium. Neurological and psychiatric adverse effects of long-term methylphenidate treatment in ADHD: A map of the current evidence. Neurosci Biobehav Rev. 2019 Sep 20; DOI: 10.1016/j.neubiorev.2019.09.023.

386. Solanto M V. Dopamine dysfunction in AD/HD: Integrating clinical and basic neuroscience research. In: Behavioural Brain Research. Behav Brain Res; 2002. p. 65-71. DOI: 10.1016/S0166-4328(01)00431-4.

387. Shaywitz BA, Yager RD, Klopper JH. Selective brain dopamine depletion in developing rats: An experimental model of minimal brain dysfunction. Science (80- ). 1976;191(4224):305-8. DOI: 10.1126/science.942800.

388. Bouchatta O, Manouze H, Bouali-Benazzouz R, Kerekes N, Ba-M'hamed S, Fossat P, Landry M, Bennis M. Neonatal 6-OHDA lesion model in mouse induces Attention-Deficit/ Hyperactivity Disorder (ADHD)-like behaviour. Sci Rep. 2018;8(1):15349. DOI: 10.1038/s41598-018-33778-0.

389. Avale ME, Falzone TL, Gelman DM, Low MJ, Grandy DK, Rubinstein M. The dopamine D4 receptor is essential for hyperactivity and impaired behavioral inhibition in a mouse model of attention deficit/hyperactivity disorder. Mol Psychiatry. 2004;9(7):718-26. DOI: 10.1038/sj.mp.4001474.

390. Dunn GA, Nigg JT, Sullivan EL. Neuroinflammation as a risk factor for attention deficit hyperactivity disorder. Vol. 182, Pharmacology Biochemistry and Behavior. Elsevier Inc.; 2019. p. 22-34. DOI: 10.1016/j.pbb.2019.05.005.

391. Leffa DT, Torres ILS, Rohde LA. A review on the role of inflammation in attention-deficit/hyperactivity disorder. Vol. 25, NeuroImmunoModulation. S. Karger AG; 2019. p. 328-33. DOI: 10.1159/000489635.

392. Miyazaki C, Koyama M, Ota E, Swa T, Mlunde LB, Amiya RM, Tachibana Y, Yamamoto-Hanada K, Mori R. Allergic diseases in children with attention deficit hyperactivity disorder: A systematic review and metaanalysis. BMC Psychiatry. 2017 Mar 31;17(1). DOI: 10.1186/s12888-017-1281-7.

393. Schans J van der, Çiçek R, de Vries TW, Hak E, Hoekstra PJ. Association of atopic diseases and attentiondeficit/hyperactivity disorder: A systematic review and meta-analyses. Vol. 74, Neuroscience and Biobehavioral Reviews. Elsevier Ltd; 2017. p. 139-48. DOI: 10.1016/j.neubiorev.2017.01.011.

394. Nielsen PR, Benros ME, Dalsgaard S. Associations Between Autoimmune Diseases and AttentionDeficit/Hyperactivity Disorder: A Nationwide Study. J Am Acad Child Adolesc Psychiatry. 2017 Mar 1;56(3):234-240.e1. DOI: 10.1016/j.jaac.2016.12.010.

395. Darwish AH, Elgohary TM, Nosair NA. Serum Interleukin-6 Level in Children With Attention-Deficit Hyperactivity Disorder (ADHD). J Child Neurol. 2019 Feb 1;34(2):61-7. DOI: 10.1177/0883073818809831.

396. Drtilkova I, Sery O, Theiner P, Uhrova A, Zackova M, Balastikova B, Znojil V. Clinical and molecular-genetic markers of ADHD in children. Neuro Endocrinol Lett. 2008 Jun;29(3):320-7.

397. Ceylan M, Sener S, Bayraktar AC, Kavutcu M. Oxidative imbalance in child and adolescent patients with attention-deficit/hyperactivity disorder. Prog Neuro-Psychopharmacology Biol Psychiatry. 2010 Dec 1;34(8):1491-4. DOI: 10.1016/j.pnpbp.2010.08.010.

398. Bulut M, Selek S, Gergerlioglu HS, Savas HA, Yilmaz HR, Yuce M, Ekici G. Malondialdehyde levels in adult attention-deficit hyperactivity disorder. J Psychiatry Neurosci. 2007;32(6):435-8. 
399. Selek S, Savas HA, Gergerlioglu HS, Bulut M, Yilmaz HR. Oxidative imbalance in adult attention deficit/hyperactivity disorder. Biol Psychol. 2008 Oct;79(2):256-9. DOI: 10.1016/j.biopsycho.2008.06.005.

400. Vogel SWN, Bijlenga D, Verduijn J, Bron TI, Beekman ATF, Kooij JJS, Penninx BWJH. Attentiondeficit/hyperactivity disorder symptoms and stress-related biomarkers. Psychoneuroendocrinology. 2017 May 1;79:31-9. DOI: 10.1016/j.psyneuen.2017.02.009.

401. Rafiepour K, Esmaeili-Mahani S, Salehzadeh A, Sheibani V. Phytohormone Abscisic Acid Protects Human Neuroblastoma SH-SY5Y Cells Against 6-Hydroxydopamine-Induced Neurotoxicity Through Its Antioxidant and Antiapoptotic Properties. Rejuvenation Res. 2019 Apr 1;22(2):99-108. DOI: 10.1089/rej.2018.2062.

402. Longe SE, Wise R, Bantick S, Lloyd D, Johansen-Berg H, McGlone F, Tracey I. Counter-stimulatory effects on pain perception and processing are significantly altered by attention: An fMRI study. Neuroreport. $2001 \mathrm{Jul}$ 3;12(9):2021-5. DOI: 10.1097/00001756-200107030-00047.

403. Liu MG, Chen J. Preclinical research on pain comorbidity with affective disorders and cognitive deficits: Challenges and perspectives. Vol. 116, Progress in Neurobiology. Elsevier Ltd; 2014. p. 13-32. DOI: 10.1016/j.pneurobio.2014.01.003.

404. Asztély K, Kopp S, Gillberg C, Waern M, Bergman S. Chronic pain and health-related quality of life in women with autism And/or ADHD: A prospective longitudinal study. J Pain Res. 2019;12:2925-32. DOI: 10.2147/JPR.S212422.

405. Kasahara S, Niwa SI, Matsudaira K, Sato N, Oka H, Yamada Y. Attention-Deficit/Hyperactivity Disorder and Chronic Pain. Vol. 82, Psychosomatic Medicine. Lippincott Williams and Wilkins; 2020. p. 346-7. DOI: 10.1097/PSY.0000000000000789.

406. Stray LL, Kristensen $\varnothing$, Lomeland M, Skorstad M, Stray T, Tønnessen FE. Motor regulation problems and pain in adults diagnosed with ADHD. Behav Brain Funct. 2013 May 3;9(1). DOI: 10.1186/1744-9081-9-18.

407. Treister R, Eisenberg E, Demeter N, Pud D. Alterations in pain response are partially reversed by methylphenidate (Ritalin) in adults with attention deficit hyperactivity disorder (ADHD). Pain Pract. 2015 Jan 1;15(1):4-11. DOI: 10.1111/papr.12129.

408. Lipsker CW, Bölte S, Hirvikoski T, Lekander M, Holmström L, Wicksell RK. Prevalence of autism traits and attention-deficit hyperactivity disorder symptoms in a clinical sample of children and adolescents with chronic pain. J Pain Res. 2018;11:2827-36. DOI: 10.2147/JPR.S177534.

409. Patros CHG, Alderson RM, Kasper LJ, Tarle SJ, Lea SE, Hudec KL. Choice-impulsivity in children and adolescents with attention-deficit/hyperactivity disorder (ADHD): A meta-analytic review. Vol. 43, Clinical Psychology Review. Elsevier Inc.; 2016. p. 162-74. DOI: 10.1016/j.cpr.2015.11.001.

410. Ünsel Bolat G, Ercan ES, Salum GA, Bilaç Ö, Massuti R, Uysal Özaslan T, Bolat H, Rohde LA. Validity of proposed DSM-5 ADHD impulsivity symptoms in children. Eur Child Adolesc Psychiatry. 2016 Oct 1;25(10):1121-32. DOI: 10.1007/s00787-016-0839-0.

411. Watterson E, Spitzer A, Watterson LR, Brackney RJ, Zavala AR, Olive MF, Sanabria F. Nicotine-induced behavioral sensitization in an adult rat model of attention deficit/hyperactivity disorder (ADHD). Behav Brain Res. 2016 Oct 1;312:333-40. DOI: 10.1016/j.bbr.2016.06.050.

412. James RS, Sharp WS, Bastain TM, Lee PP, Walter JM, Czarnolewski M, Xavier Castellanos F. Double-blind, placebo-controlled study of single-dose amphetamine formulations in ADHD. J Am Acad Child Adolesc Psychiatry. 2001;40(11):1268-76. DOI: 10.1097/00004583-200111000-00006.

413. Stickley A, Koyanagi A, Takahashi H, Kamio Y. ADHD symptoms and pain among adults in England. Psychiatry Res. 2016 Dec 30;246:326-31. DOI: 10.1016/j.psychres.2016.10.004.

414. Scherder EJA, Rommelse NNJ, Bröring T, Faraone S V., Sergeant JA. Somatosensory functioning and experienced pain in ADHD-families: A pilot study. Eur J Paediatr Neurol. 2008 Nov;12(6):461-9. DOI: 10.1016/j.ejpn.2007.11.004.

415. Lei H, Ren R, Sun Y, Zhang K, Zhao X, Ablat N, Pu X. Neuroprotective Effects of Safflower Flavonoid Extract in 6-Hydroxydopamine-Induced Model of Parkinson's Disease May Be Related to its Anti-Inflammatory Action. Molecules. 2020 Nov 9;25(21). DOI: 10.3390/molecules25215206.

416. Schober A. Classic toxin-induced animal models of Parkinson's disease: 6-OHDA and MPTP. Vol. 318, Cell and Tissue Research. Cell Tissue Res; 2004. p. 215-24. DOI: 10.1007/s00441-004-0938-y.

417. Wang GJ, Volkow ND, Logan J, Pappas NR, Wong CT, Zhu W, Netusll N, Fowler JS. Brain dopamine and obesity. Lancet. 2001 Feb 3;357(9253):354-7. DOI: 10.1016/S0140-6736(00)03643-6. 
418. de Weijer BA, van de Giessen E, van Amelsvoort TA, Boot E, Braak B, Janssen IM, van de Laar A, Fliers E, Serlie MJ, Booij J. Lower striatal dopamine D2/3 receptor availability in obese compared with non-obese subjects. EJNMMI Res. 2011;1(1):1-5. DOI: 10.1186/2191-219X-1-37.

419. MohanKumar SMJ, Kasturi BS, Shin AC, Balasubramanian P, Gilbreath ET, Subramanian M, MohanKumar PS. Chronic estradiol exposure induces oxidative stress in the hypothalamus to decrease hypothalamic dopamine and cause hyperprolactinemia. Am J Physiol - Regul Integr Comp Physiol. 2011 Mar;300(3). DOI: 10.1152/ajpregu.00481.2010.

420. Ogata M, Akita H, Ishibashi H. Behavioral responses to anxiogenic tasks in young adult rats with neonatal dopamine depletion. Physiol Behav. 2019 May 15;204:10-9. DOI: 10.1016/j.physbeh.2019.02.006.

421. Liu ZJ, Liu W, Liu L, Xiao C, Wang Y, Jiao JS. Curcumin protects neuron against cerebral ischemia-induced inflammation through improving PPAR-gamma function. Evidence-based Complement Altern Med. 2013;2013. DOI: $10.1155 / 2013 / 470975$

422. Schiborr C, Kocher A, Behnam D, Jandasek J, Toelstede S, Frank J. The oral bioavailability of curcumin from micronized powder and liquid micelles is significantly increased in healthy humans and differs between sexes. Mol Nutr Food Res. 2014 Mar;58(3):516-27. DOI: 10.1002/mnfr.201300724.

423. Laakso A, Vilkman H, Bergman J, Haaparanta M, Solin O, Syvälahti E, Salokangas RKR, Hietala J. Sex differences in striatal presynaptic dopamine synthesis capacity in healthy subjects. Biol Psychiatry. 2002 Oct 1;52(7):759-63. DOI: 10.1016/S0006-3223(02)01369-0.

424. McKinnon B, Bersinger NA, Huber AW, Kuhn A, Mueller MD. PPAR- $\gamma$ expression in peritoneal endometriotic lesions correlates with pain experienced by patients. Fertil Steril. 2010 Jan 1;93(1):293-6. DOI: 10.1016/j.fertnstert.2009.07.980.

425. Gold CA, Budson AE. Memory loss in Alzheimer's disease: Implications for development of therapeutics. Vol. 8, Expert Review of Neurotherapeutics. NIH Public Access; 2008. p. 1879-91. DOI: $10.1586 / 14737175.8 .12 .1879$

426. Consoli A, Devangelio E. Thiazolidinediones and inflammation. Vol. 14, Lupus. Lupus; 2005. p. 794-7. DOI: 10.1191/0961203305lu2223oa.

427. Cha J, Greenberg T, Song I, Blair Simpson H, Posner J, Mujica-Parodi LR. Abnormal hippocampal structure and function in clinical anxiety and comorbid depression. Hippocampus. 2016 May 1;26(5):545-53. DOI: 10.1002/hipo.22566.

428. Campbell S, MacQueen G. The role of the hippocampus in the pathophysiology of major depression. Vol. 29, Journal of Psychiatry and Neuroscience. Canadian Medical Association; 2004. p. 417-26.

429. Bannerman DM, Grubb M, Deacon R, Yee B, Feldon J, Rawlins J. Ventral hippocampal lesions affect anxiety but not spatial learning. Behav Brain Res. 2003 Mar 1;139:197-213. DOI: 10.1016/S0166-4328(02)00268-1.

430. Man HY, Lin JW, Ju WH, Ahmadian G, Liu L, Becker LE, Sheng M, Wang YT. Regulation of AMPA receptormediated synaptic transmission by clathrin-dependent receptor internalization. Neuron. 2000;25(3):649-62. DOI: 10.1016/S0896-6273(00)81067-3.

431. Chiu SL, Cline HT. Insulin receptor signaling in the development of neuronal structure and function. Vol. 5, Neural Development. Neural Dev; 2010. DOI: 10.1186/1749-8104-5-7.

432. Ferrario CR, Reagan LP. Insulin-mediated synaptic plasticity in the CNS: Anatomical, functional and temporal contexts. Vol. 136, Neuropharmacology. Elsevier Ltd; 2018. p. 182-91. DOI: 10.1016/j.neuropharm.2017.12.001.

433. Liu Z, Patil IY, Jiang T, Sancheti H, Walsh JP, Stiles BL, Yin F, Cadenas E. High-fat diet induces hepatic insulin resistance and impairment of synaptic plasticity. PLoS One. 2015 May 29;10(5). DOI: 10.1371/journal.pone.0128274.

434. Linn MC, Petersen AC. Emergence and characterization of sex differences in spatial ability: a meta-analysis. Child Dev. 1985 Dec;56(6):1479-98.

435. Jonasson Z. Meta-analysis of sex differences in rodent models of learning and memory: A review of behavioral and biological data. Vol. 28, Neuroscience and Biobehavioral Reviews. Neurosci Biobehav Rev; 2005. p. 81125. DOI: 10.1016/j.neubiorev.2004.10.006.

436. Gale SD, Baxter L, Thompson J. Greater memory impairment in dementing females than males relative to sexmatched healthy controls. $J$ Clin Exp Neuropsychol. 2016 May 27;38(5):527-33. DOI: 10.1080/13803395.2015.1132298. 
437. Hua X, Hibar DP, Lee S, Toga AW, Jack CR, Weiner MW, Thompson PM. Sex and age differences in atrophic rates: An ADNI study with $\mathrm{n}=1368$ MRI scans. Neurobiol Aging. 2010 Aug;31(8):1463-80. DOI: 10.1016/j.neurobiolaging.2010.04.033.

438. Ducluzeau PH, Cousin P, Malvoisin E, Bornet H, Vidal H, Laville M, Pugeat M. Glucose-to-insulin ratio rather than sex hormone-binding globulin and adiponectin levels is the best predictor of insulin resistance in nonobese women with polycystic ovary syndrome. J Clin Endocrinol Metab. 2003 Aug 1;88(8):3626-31. DOI: $10.1210 /$ jc.2003-030219.

439. May AA, Bedel ND, Shen L, Woods SC, Liu M. Estrogen and insulin transport through the blood-brain barrier. Physiol Behav. 2016 Sep 1;163:312-21. DOI: 10.1016/j.physbeh.2016.05.019.

440. Klinge CM. Estrogenic control of mitochondrial function and biogenesis. Vol. 105, Journal of Cellular Biochemistry. J Cell Biochem; 2008. p. 1342-51. DOI: 10.1002/jcb.21936.

441. Tada H, Koide M, Ara W, Shibata Y, Funabashi T, Suyama K, Goto T, Takahashi T. Estrous cycle-dependent phasic changes in the stoichiometry of hippocampal synaptic AMPA receptors in rats. PLoS One. 2015 Jun 29;10(6). DOI: 10.1371/journal.pone.0131359.

442. Woolley CS, Gould E, Frankfurt M, McEwen BS. Naturally occurring fluctuation in dendritic spine density on adult hippocampal pyramidal neurons. J Neurosci. 1990;10(12):4035-9. DOI: 10.1523/jneurosci.10-1204035.1990.

443. Good M, Day M, Muir JL. Cyclical changes in endogenous levels of oestrogen modulate the induction of LTD and LTP in the hippocampal CA1 region. Eur J Neurosci. 1999 Dec;11(12):4476-80. DOI: 10.1046/j.14609568.1999.00920.x.

444. Liu Y, Ho RCM, Mak A. Interleukin (IL)-6, tumour necrosis factor alpha (TNF- $\alpha$ ) and soluble interleukin-2 receptors (sIL-2R) are elevated in patients with major depressive disorder: A meta-analysis and meta-regression. Vol. 139, Journal of Affective Disorders. J Affect Disord; 2012. p. 230-9. DOI: 10.1016/j.jad.2011.08.003.

445. Rao JS, Harry GJ, Rapoport SI, Kim HW. Increased excitotoxicity and neuroinflammatory markers in postmortem frontal cortex from bipolar disorder patients. Mol Psychiatry. 2010 Apr;15(4):384-92. DOI: 10.1038/mp.2009.47.

446. Isbrandt D. A mechanistic link between glia and neuronal excitability in acute neuroinflammation. $J$ Physiol. 2017 Feb 1;595(3):603-4. DOI: 10.1113/JP273252.

447. Ji RR, Nackley A, Huh Y, Terrando N, Maixner W. Neuroinflammation and central sensitization in chronic and widespread pain. Anesthesiology. 2018 Aug 1;129(2):343-66. DOI: 10.1097/ALN.0000000000002130.

448. Ji RR, Nackley A, Huh Y, Terrando N, Maixner W. Neuroinflammation and central sensitization in chronic and widespread pain. Anesthesiology. 2018 Aug 1;129(2):343-66. DOI: 10.1097/ALN.0000000000002130.

449. Allard MJ, Brochu ME, Bergeron JD, Sebire G. Hyperactive behavior in female rats in utero-exposed to group B Streptococcus-induced inflammation. Int $J$ Dev Neurosci. 2018 Oct 1;69:17-22. DOI: 10.1016/j.ijdevneu.2018.06.005.

450. Vohr BR, Davis EP, Wanke CA, Krebs NF. Neurodevelopment: The impact of nutrition and inflammation during preconception and pregnancy in low-resource settings. Pediatrics. 2017 Apr 1;139(Suppl 1):S38-49. DOI: $10.1542 /$ peds.2016-2828F.

451. Allred EN, Dammann O, Fichorova RN, Hooper SR, Hunter SJ, Joseph RM, Kuban K, Leviton A, O’Shea TM, Scott MN. Systemic Inflammation during the First Postnatal Month and the Risk of Attention Deficit Hyperactivity Disorder Characteristics among 10 year-old Children Born Extremely Preterm. J Neuroimmune Pharmacol. 2017 Sep 1;12(3):531-43. DOI: 10.1007/s11481-017-9742-9.

452. Reyero F, Ponce G, Rodriguez-Jimenez R, Fernandez-Dapica P, Taboada D, Martin V, Navio M, JimenezArriero MA, Hoenicka J, Palomo T. High frequency of childhood ADHD history in women with fibromyalgia. Eur Psychiatry. 2011 Nov;26(8):482-3. DOI: 10.1016/j.eurpsy.2010.03.012.

453. Wiwe Lipsker C, von Heijne M, Bölte S, Wicksell RK. A case report and literature review of autism and attention deficit hyperactivity disorder in paediatric chronic pain. Acta Paediatr Int J Paediatr. 2018 May 1;107(5):753-8. DOI: 10.1111/apa.14220.

454. Treister R, Eisenberg E, Demeter N, Pud D. Alterations in pain response are partially reversed by methylphenidate (Ritalin) in adults with attention deficit hyperactivity disorder (ADHD). Pain Pract. 2015 Jan 1;15(1):4-11. DOI: 10.1111/papr.12129.

455. Stray LL, Kristensen Ø, Lomeland M, Skorstad M, Stray T, Tønnessen FE. Motor regulation problems and pain 
in adults diagnosed with ADHD. Behav Brain Funct. 2013 May 3;9(1). DOI: 10.1186/1744-9081-9-18.

456. Mowlem FD, Rosenqvist MA, Martin J, Lichtenstein P, Asherson P, Larsson H. Sex differences in predicting ADHD clinical diagnosis and pharmacological treatment. Eur Child Adolesc Psychiatry. 2019 Apr 1;28(4):4819. DOI: $10.1007 / \mathrm{s} 00787-018-1211-3$

457. Rucklidge JJ. Gender Differences in Attention-Deficit/Hyperactivity Disorder. Vol. 33, Psychiatric Clinics of North America. Psychiatr Clin North Am; 2010. p. 357-73. DOI: 10.1016/j.psc.2010.01.006.

458. Weiss M, Worling D, Wasdell M. A chart review study of the Inattentive and Combined Types of ADHD. Vol. 7, Journal of Attention Disorders. SAGE Publications Inc.; 2003. p. 1-9. DOI: 10.1177/108705470300700101.

459. Felger JC, Abe T, Kaunzner UW, Gottfried-Blackmore A, Gal-Toth J, McEwen BS, Iadecola C, Bulloch K. Brain dendritic cells in ischemic stroke: Time course, activation state, and origin. Brain Behav Immun. 2010 Jul;24(5):724-37. DOI: 10.1016/j.bbi.2009.11.002.

460. Ginhoux F, Greter M, Leboeuf M, Nandi S, See P, Gokhan S, Mehler MF, Conway SJ, Ng LG, Stanley ER, Samokhvalov IM, Merad M. Fate mapping analysis reveals that adult microglia derive from primitive macrophages. Science (80- ). 2010 Nov 5;330(6005):841-5. DOI: 10.1126/science.1194637.

461. Hanamsagar R, Bilbo SD. Sex differences in neurodevelopmental and neurodegenerative disorders: Focus on microglial function and neuroinflammation during development. Vol. 160, Journal of Steroid Biochemistry and Molecular Biology. Elsevier Ltd; 2016. p. 127-33. DOI: 10.1016/j.jsbmb.2015.09.039. 
10

ANNEXES 


\subsection{Supplementary information}

\subsubsection{Cell transfection and RT-qPCR}

HEK cells were grown at $37^{\circ} \mathrm{C}$ in a $5 \% \mathrm{CO}_{2}$ incubator and $90 \%$ humidity and were free of mycoplasma contamination. Cells were transfected by the calcium chloride method with plasmids control pSM155-CMV-EGFP generous donation of Dr Melanie White and our generated pSM155CMV-shIRS1-EGFP with two different sequences (shIRS1A, shIRS1B). To quantify the knockdown capacity of the shRNA candidates, we transfected the HEK293 with the shRNA containing plasmids using the calcium phosphate method and measure the IRS1 and IRS2 expression using real-time PCR. GAPDH was used as an internal control. The sequences of the primers used are depicted in Supplementary Table S5.2. 


\subsection{Supplementary figures}

\section{Sh-IRS1A}

Top

5'TGCTGGCAGGCACCATCTCAACAATCGTTTTGGCCACTGACTGACGATTGTTGAGA TGGTGCCTGC 3'

\section{Bottom}

5'CCTGGCAGGCACCATCTCAACAATCGTCAGTCAGTGGCCAAAACGATTGTTGAGAT GGTGCCTGCC 3'

\section{Sh-IRS1B}

Top 5`TGCTGGCCTGGAGTATTATGAGAACGGTTTTGGCCACTGACTGACCGTTCTCATAA TACTCCAGGC 3'

\section{Bottom}

5'CCTGGCCTGGAGTATTATGAGAACGGTCAGTCAGTGGCCAAAACCGTTCTCATAAT ACTCCAGGCC3'

Fig. S5.1. Sequences of the oligonucleotides used for the two shRNA constructs selected as shIRS1 candidates. 


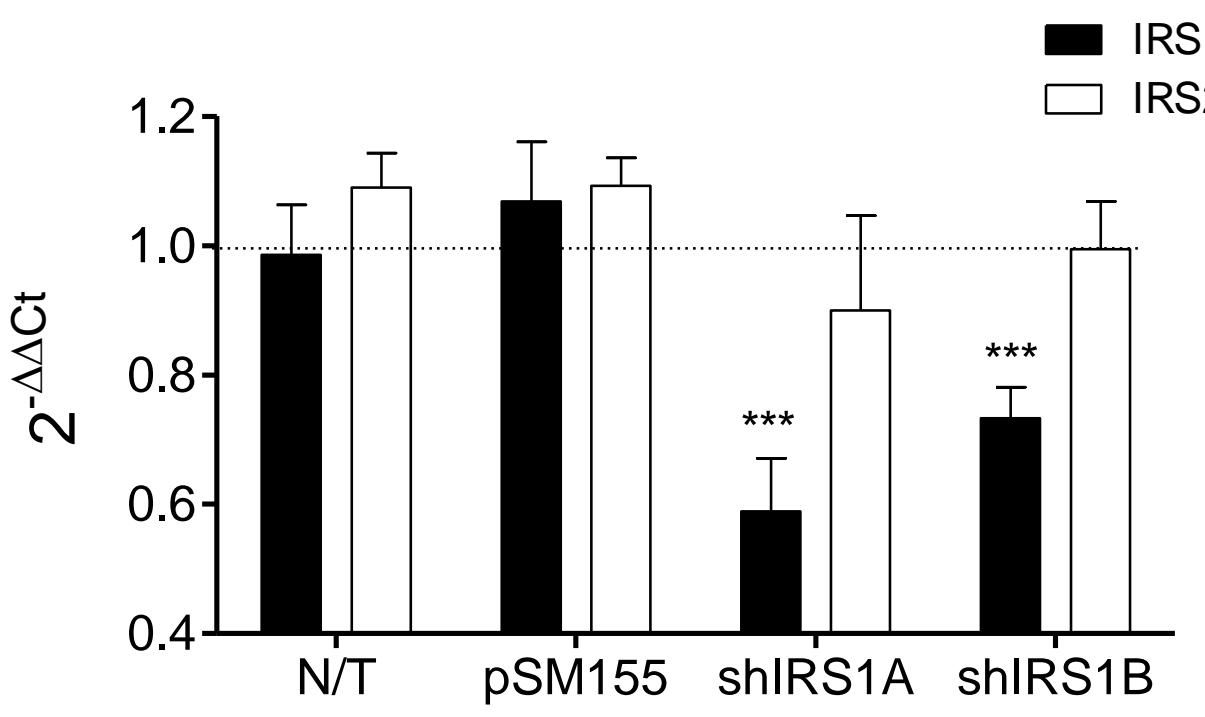

Fig. S5.2. Silencing effect of shIRS1 in cultured cells. Human embryonic kidney cells (HEK 293) were transfected with the different plasmids as indicated. IRS1 and IRS2 mRNA expression was quantified by qPCR. Data were analyzed using two tailed Mann Whitney t-test; *** $\mathrm{p}<0.001$ compared to control plasmid pSM155. 

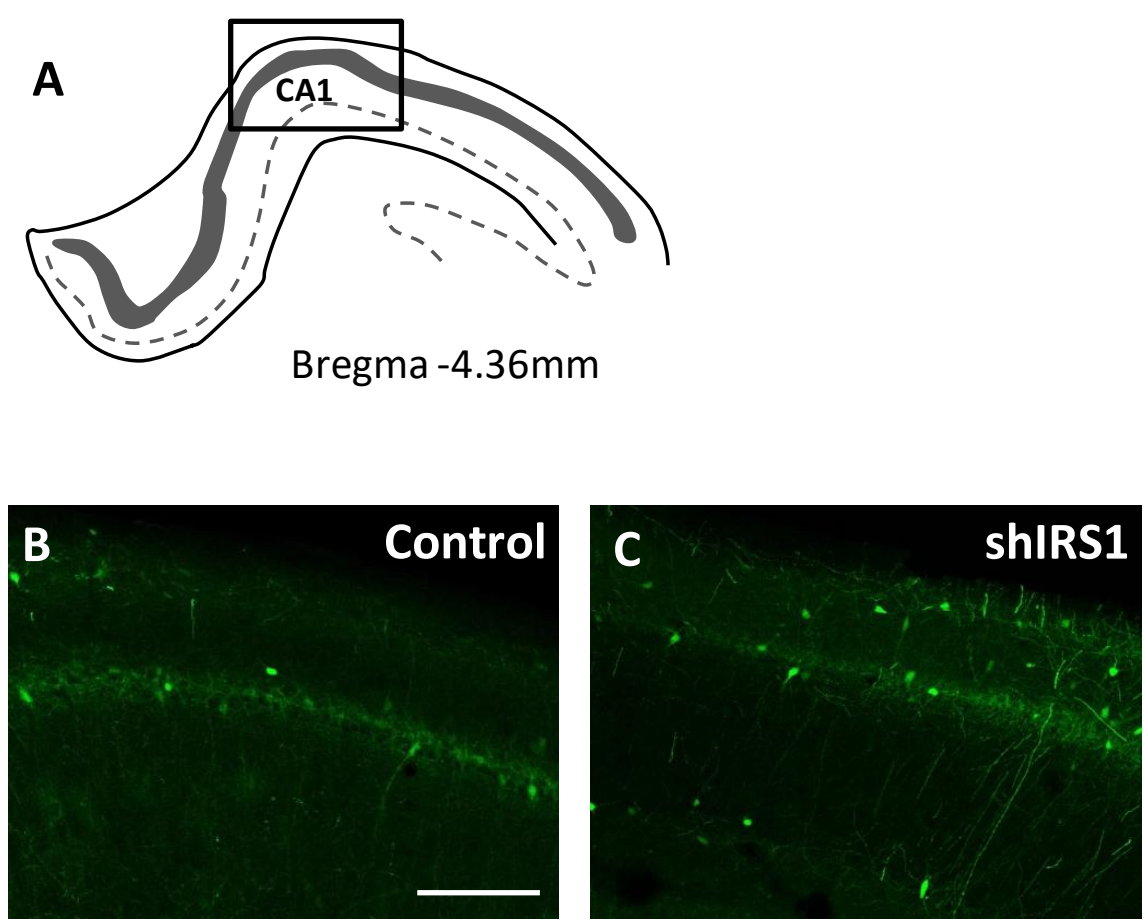

Fig. S5.3. AAV in the hippocampus CA1 field. Schematic representation of CA1 of the dorsal hippocampus (A). Scattered control (B) and shIRS1 (C) labeled neurons were observed in the hippocampus CA1. Scale bar $=100 \mu \mathrm{m}(\mathrm{n}=3-5)$. 

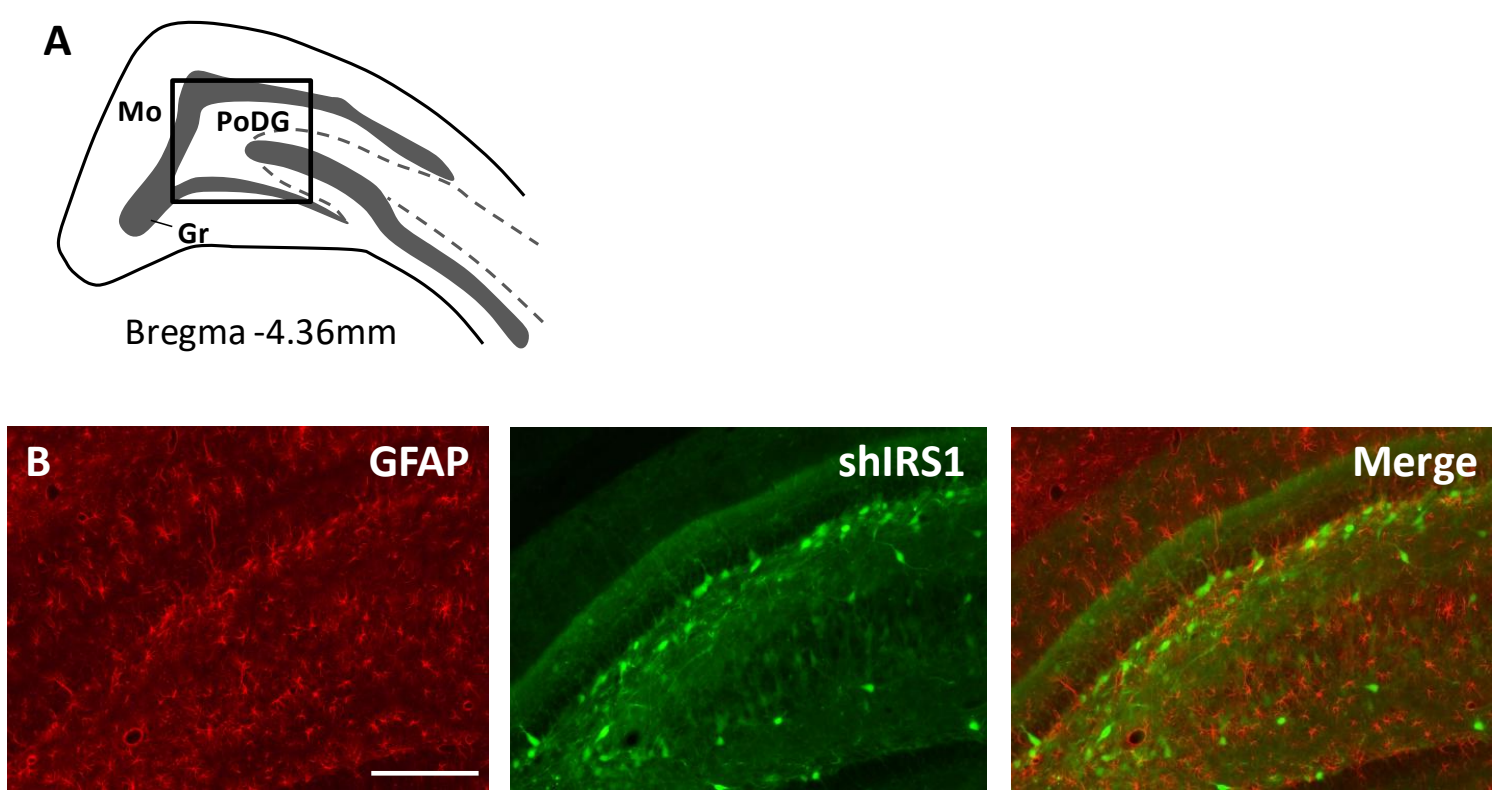

Fig. S5.4. AAV DJ8 does not colocalize with GFAP-positive astrocytes. Schematic representation of the dentate gyrus of the dorsal hippocampus (A). We found no co-localization of AAV labeled cells with Glial Fibrillary Acidic Protein (GFAP) positive cells (B). Scale bar=100 $\mu \mathrm{m}(\mathrm{n}=3-4)$. 


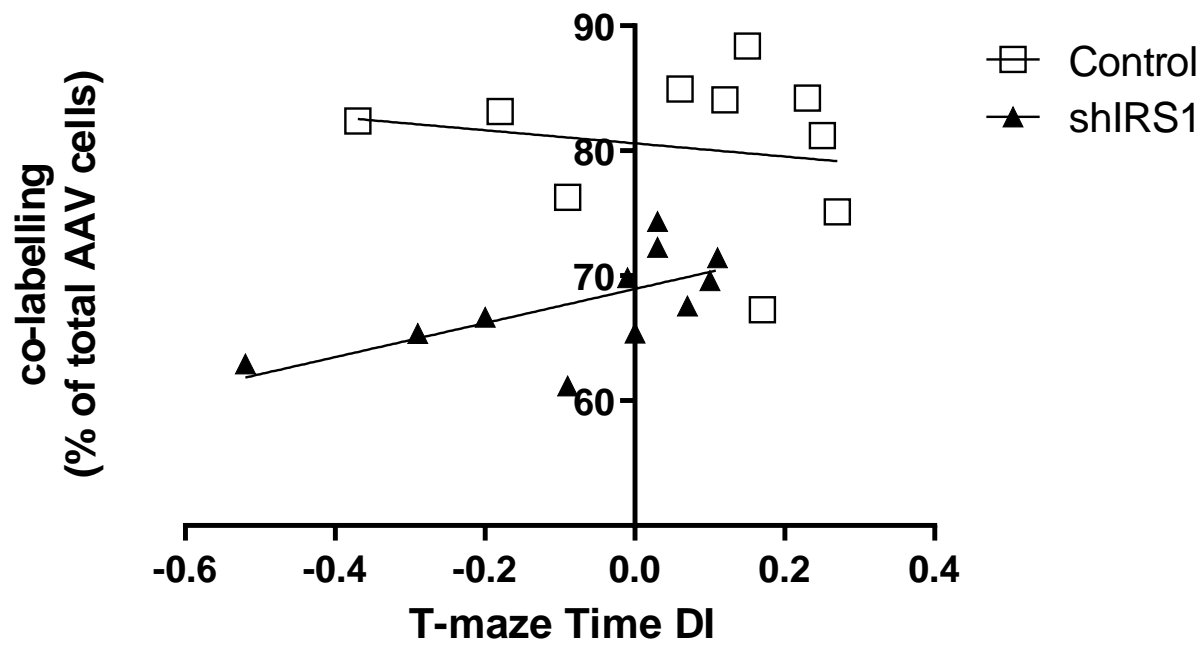

\begin{tabular}{lll}
\hline & Time DI vs Control & Time DI vs shIRS1 \\
\hline Pearson $r(p$ value $)$ & $-0.15(p=0,34)$ & $0.63(p=0,018)$ \\
Slope significant from zero & No & Yes \\
\hline
\end{tabular}

Fig. S5.5. Correlation between T-maze behaviour (DI) and IRS1 knockdown was compared between control subjects (squares) and shIRS1 (triangles). Pearson $r$ values and probability are indicated, only in shIRS1-inoculated animals, behaviour correlated positively with IRS1 expression correlates $p$ Regression analysis indicated that the slope is significantly different from zero only in shIRS1-inoculated subjects. 

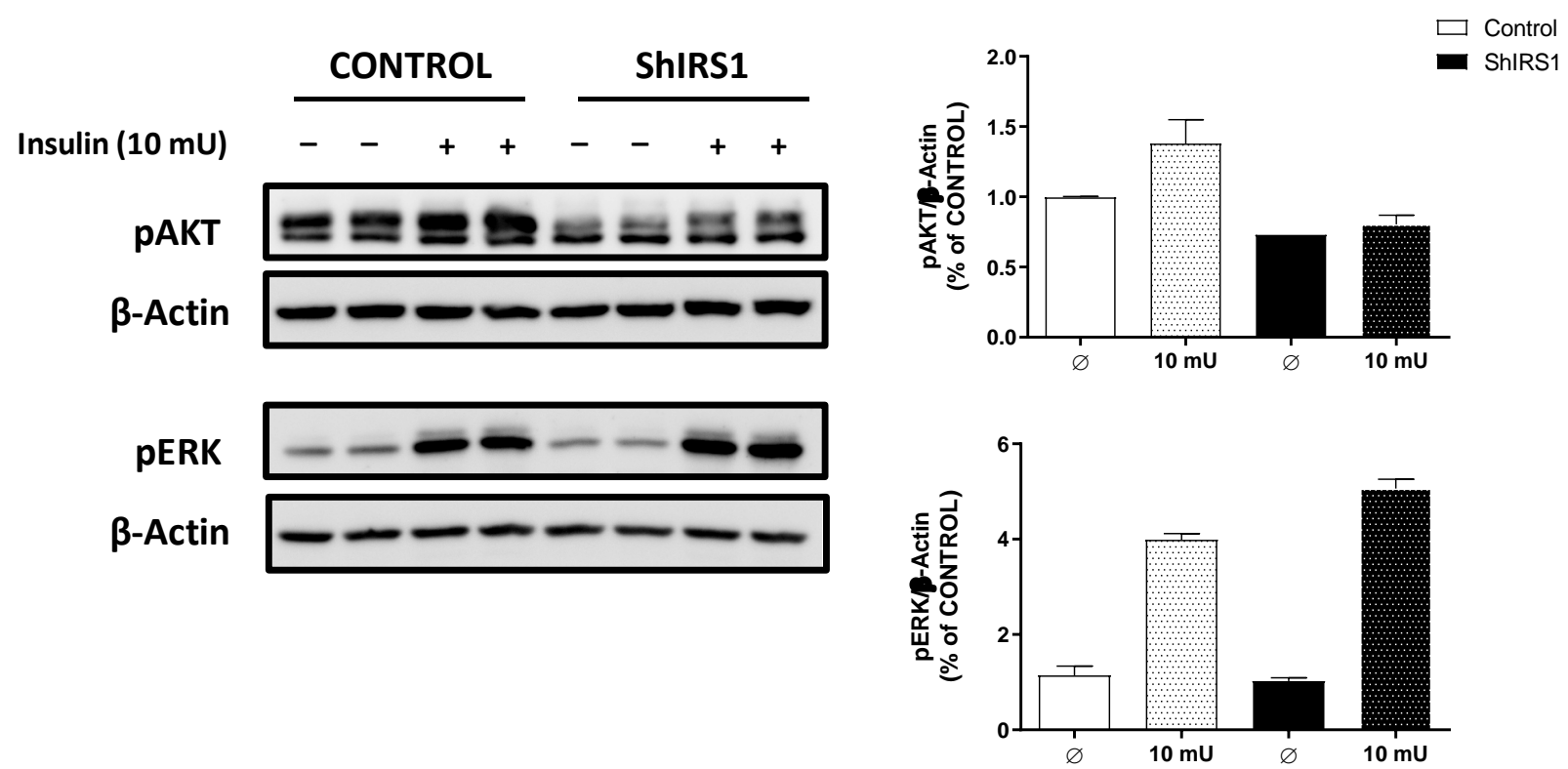

Fig. S5.6. HEK cells were transfected with shIRS1 plasmid or control plasmid. Insulin was added for $5 \mathrm{~min}$ and cells were collected. The AAV shIRS1 reduced levels of phosphorylated AKT (pAKT) basal and activation. pAKT: phosphorylated AKT; pERK: phosphorylated extracellular signal-regulated kinase. Unpublished results. 


\subsection{Supplementary tables}

Table S5.1. Animals were weighed before surgery (Day 0) and one month after (Day 33), just prior to behavior. Subjects, females and males were classified randomly into control AAV-CMVEGFP and AAV-CMV-shIRS1-EGFP experimental groups.

\begin{tabular}{ccccc}
\hline Rat & Sex & $\begin{array}{c}\text { Experimental } \\
\text { group }\end{array}$ & $\begin{array}{c}\text { Weight prior to } \\
\text { surgery (g) }\end{array}$ & $\begin{array}{c}\text { Weight 33 days after surgery } \\
\text { (prior to behavior) (g) }\end{array}$ \\
\hline $\mathbf{1}$ & F & Control & 331 & 332 \\
$\mathbf{2}$ & F & Control & 288 & 303 \\
$\mathbf{3}$ & F & Control & 308 & 316 \\
$\mathbf{4}$ & F & Control & 299 & 313 \\
$\mathbf{5}$ & F & Control & 329 & 330 \\
$\mathbf{6}$ & F & Control & 285 & 310 \\
$\mathbf{7}$ & F & Control & 293 & 293 \\
$\mathbf{8}$ & F & ShIRS1 & 289 & 286 \\
$\mathbf{9}$ & F & ShIRS1 & 315 & 315 \\
$\mathbf{1 0}$ & F & ShIRS1 & 298 & 299 \\
$\mathbf{1 1}$ & F & ShIRS1 & 263 & 279 \\
$\mathbf{1 2}$ & F & ShIRS1 & 335 & 344 \\
$\mathbf{1 3}$ & F & ShIRS1 & 344 & 340 \\
$\mathbf{1 4}$ & F & ShIRS1 & 295 & 293 \\
$\mathbf{1 5}$ & F & ShIRS1 & 306 & 312 \\
$\mathbf{1}$ & M & Control & 460 & 503 \\
\hline $\mathbf{2}$ & M & Control & 485 & 565 \\
$\mathbf{3}$ & M & Control & 523 & 572 \\
$\mathbf{4}$ & M & Control & 455 & 540 \\
$\mathbf{5}$ & M & Control & 495 & 536 \\
\hline $\mathbf{6}$ & M & Control & 463 & 505 \\
$\mathbf{7}$ & M & Control & 450 & 527 \\
$\mathbf{8}$ & M & Control & 477 & 542 \\
$\mathbf{9}$ & M & ShIRS1 & 482 & 539 \\
$\mathbf{1 0}$ & M & ShIRS1 & 478 & 566 \\
$\mathbf{1 1}$ & M & ShIRS1 & 535 & 600 \\
$\mathbf{1 2}$ & M & ShIRS1 & 463 & 511 \\
$\mathbf{1 3}$ & M & ShIRS1 & 494 & 577 \\
$\mathbf{1 4}$ & M & ShIRS1 & 517 & \\
$\mathbf{1 5}$ & M & ShIRS1 & 503 & \\
\hline & & & & \\
\hline
\end{tabular}

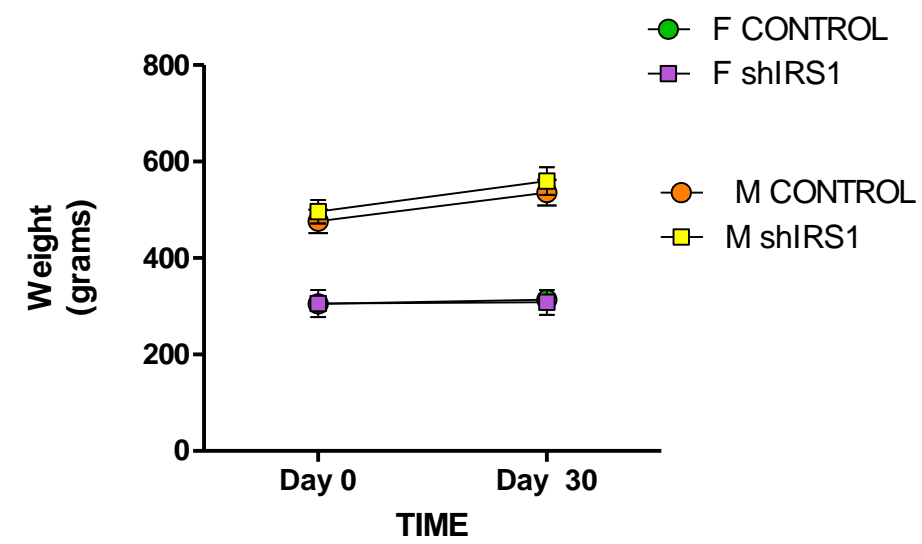


Table S5.2. Primers sequences obtained using Primer3 software tool.

Gene Accession number Forward Primer (5'->3') Reverse Primer (5' $->3$ ')

GAPDH NM_017008.4 TGCCCCCATGTTTGTGATG $\quad$ TGGTGGTGCAGGATGCATT

IRS-1 NM_012969.1 ATTGGAGGTGGGTCTTGCAG TGGGGATCTTCTGGGCCATA

IRS-2 NM_001168633.1 TCACCACAGGACACAGATGC GCATGAAGTGTGGCAAACGT 


\title{
9.4. Publications
}

\section{The effect of abscisic acid chronic treatment on neuroinflammatory markers and memory in a rat model of high-fat diet induced neuroinflammation}

Sandra Sánchez-Sarasúa ${ }^{1 \dagger}$, Salma Moustafa', Álvaro Gardar-Avilés', Marla Fernanda López-Climent?', Aurelio Gómez-Cadenas ${ }^{2}$, Francisco E. Oluchar-Bordonau' and Ana M. Sánchez-Pérez ${ }^{*}$

\begin{abstract}
Badkground: Western diet and lifestyle are associated with overweight, obesity, and type 2 diabetes, which, in turn, are comelated with neuroinflammation processes. Exercise and a healthy diet are important in the prevention of these disorders However, molecules inhibiting neuroinflammation might also be efficacious in the prevention and/or treatment of neurobgical disorders of inflammatory etiology. The abscisic acid (ABA) is a phytohormone irvolved in hydric-stress responses This compound is not only found in plants but also in other organisms including mammals. In rodents, ABA can play a beneficial role in the regulation of peripheral immune response and insulin action. Thus we

hypothesized that chronic ABA administration might exert a protective effect in a model of neuroinflammation induced by high-fat diet (HFD).

Methods: Male Wistar rats were fed with standard diet or HFD with or without ABA in the drinking water for 12 weeks Glucose tolerance test and behavioral paradigms were performed to evaluate the peripheral and central effects of treatments One-Way ANOVA was performed analyzed statistical differences between groups.

Results: The HFD induced insulin resistance peripherally and increased the levels of proinflammatory markers in in the brain. We observed that ABA restored glucose tolerance in HFD-fed rats, as expected. In addition, chronic $A B A$ treatment rescued cognitive performance in these animals while not affecting control diet fed animals. Moreover, it counteracted the changes induced by HFD in the hypothalamus; microglia activations and TNFa mRNA levels.

Condusion: These results suggest that $A B A$ might become a new therapeutic molecule improving the neuroinflammatory status and insulin resistance.

Keywords: Obesity, Working memory, Microglia, Phytohormones
\end{abstract}

\section{Badkground}

Obesity, a leading cause of type 2 diabetes [1], correlates with cognitive impairment. Insulin sensitizers have been proposed as a promising tool for the reduction of obesity-induced insulin resistance and inflammation processes. The thiazolidinediones (TDZ) are a family of

\section{* Correpondence snchesnouler}

Equal contribusons

Lepantment of Moddine, Unversiy of hume l, Voente Sos Bayyas sh, tzort Gatelón de a Mans, Gartilión spain

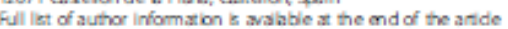

synthetic insulin sensitizer molecules; however, some of them have undesirable side effects [2-4]. Thus, alternative compounds with analogous properties but fewer side effects are needed. The phytohormone abscisic acid (ABA) was found in mammalian cells more than 25 years ago [5]. Since then, several studies have proposed it as a universal signaling molecule $[6,7]$. Structurally, ABA is very similar to the TDZs. Indeed, ABA can improve glucose tolerance $[8]$, reduce the level of $T N F \alpha$, and decrease adipocyte cell size in an in vivo model of obesity induced by HFD [9]. Moreover, in human and murine 


\title{
Abscisic Acid Supplementation Rescues High Fat Diet-Induced Alterations in Hippocampal Inflammation and IRSs Expression
}

\author{
Alberto Ribes-Navarro ${ }^{1}$ - Mariam Atef ${ }^{1}$ + Sandra Sánchez-Sarasúa ${ }^{1}$ - Maria Teresa Beltrán-Bretones ${ }^{1}$. \\ Francisco Olucha-Bordonau ' ${ }^{1}$. Ana María Sánchez-Pérez ${ }^{1}$ \\ Peceivect 9 Jnuary 2018/Accepted: 17 Aptil 2013 \\ Springer Solence+Bushess Media LLC, pat of Spinger Nature 20 18
}

\section{Abstract}

Accumulated evidence indicates that neuroinflammation induces insulin resistance in the brain. Moreover, both processes are intimately linked to neurodegenerative disorders, including Abheimer's disease. Potential mechanisms underlying insulin resistance inchude serine phosphorylation of the insulin receptor substrate (IRS) or insulin receptor (IR) misallocation. However, only a few studies have focused on IRS expression in the brain and its modulation in neuroinflammatory processes This study used the high-fat diet (HFD) model of neuroinflammation to study the alterations of IR, an insulin-like growth factor receptor (IGFIR) and IRS expressions in the hippocampus. We observed that HFD effectively reduced mRNA and protein IRS2 expression. In contrast, a HFD induced the upregulation of the IRSI mRNA levels, but did not alter an IR and IGFIR expression. As expected, we observed that a HFD increased hippocampal tumor necrosis factor alpha (TNF $\alpha$ ) and amyloid precursor protein (APP) levels while reducing brainderived neurotrophic factor (BDNF) expression and neurogenesis. Interestingly, we found that TNF $\alpha$ conelated positively with IRS I and negatively with IRS2, whereas APP levels conclated positively only with IRS1 but not IRS2. These results indicate that IRS1 and IRS2 hippocampal expression can be affectod dfferently by HFD-induced neuroinflammation. In addition, we aimod to establish whether abecisic acid (ABA) can rescue hippocampal IRS1 and IRS2 expression, as we had previously shown that ABA supplementation prevents memory impaimerts and improves neurcinflammation induced by a HFD. In this study, ABA restored HFDinduced hippocampal alterations, inchuding IRS1 and IRS2 expression. TNF $\alpha$, APP, and BDNF levels and neurogenesis In conclusion, this study highlights different regulations of hippocampal IRS1 and IRS2 expression using a HFD, indicating the important differences of these scaffolding proteins, and strongly supports ABA therapeutic effects.

Keywords Insulin resistance - Hippocampus - APP · BDNF - Neurogenesis - Neuro inflammation

$\begin{array}{ll}\text { Abbreviations } \\ \text { ABA } & \text { Abscisic acid } \\ \text { ANOVA } & \text { Analysis of variance } \\ \text { APP } & \text { Amyloid precursor protein } \\ \text { BDNF } & \text { Brain-derived neurotrophic factor } \\ \text { ERK } & \text { Extracellular regulated kinases } \\ \text { HFD } & \text { High-fat diet } \\ \text { IGF1 } & \text { Insulin-like growth factor } \\ \text { IGF1R } & \text { Insulin-like growth factor receptor } \\ \text { IR } & \text { Insulin roceptor } \\ \text { IRS } & \text { Insulin roceptor substrate }\end{array}$

A Ars Maris SänchezPére: sancheanฒuji.es

1. Dquarment of Medicine, Univessity of Jaume L, Castellón de $\mathrm{B}$ Plena Srain

\begin{tabular}{|c|c|}
\hline PFA & Paraformaldehyde \\
\hline PPAR- $\gamma$ & $\begin{array}{l}\text { Peroxisome proliferator-activated } \\
\text { receptor gamma }\end{array}$ \\
\hline RTqPCR & $\begin{array}{l}\text { Real-time quantitative polymerase } \\
\text { chain reaction }\end{array}$ \\
\hline SEM & Standard enor of mean \\
\hline SD & Standard diet \\
\hline SGZ & Subgranular zone \\
\hline TBS & Tris-buffered saline \\
\hline TDZ & Thizzolidinodiones \\
\hline $\mathrm{TNF} \alpha$ & Tumor necrosis factor alpha \\
\hline
\end{tabular}

\section{Introduction}

Adult neurogenesis is a sensitive process that is very susceptible to different toxic insults, such as the presence of beta 


\title{
AAV delivery of shRNA against IRS1 in GABAergic neurons in rat hippocampus impairs spatial memory in females and male rats
}

\author{
Sandra Sánchez-Sarasúa ${ }^{1}$ - Alberto Ribes-Navarro ${ }^{1,2}$ - María Teresa Beltrán-Bretones ${ }^{1}$ - Ana María Sánchez-Pérez ${ }^{1} \mathrm{C}$ \\ Recelved: 4 March 2020/ Accepted: 6 October 2020 \\ OSpringer-Verlag GmbH Germany, part of Springer Nature 2020
}

\section{Abstract}

Brain insulin resistance is a major factor leading to impaired cognitive function and it is considered as the onset of Alzheimer's disease. Insulin resistance is intimately linked to inflammatory conditions, many studies have revealed how pro-inflammatory cytokines lead to insulin resistance, by inhibiting IRSI function. Thus, the dysfunction of insulin signaling is concomitant with inflammatory biomarkers. However, the specific effect of IRS1 impaired function in otherwise healthy brain has not been dissected out. So, we decided in our study, to study the specific role of IRS 1 in the hippocampus, in the absence of comorbidities. To that end, shR NA against rat and human IRS1 was designed and tested in cultured HEK cells to evaluate mRNA levels and specificity. The best candidate sequence was encapsulated in an AAV vector (strain DJ8) under the control of the cytomegalowirus promoter and together with the green fluorescent protein gene as a reporter. AAV-CMV-shIRS1-EGFP and control AAV-CMV-EGFP were inoculated into the dorsal hippocampus of female and male Wistar rats. One month later, animals undertook a battery of behavioral paradigms evaluating spatial and social memory and anxiety. Our results suggest that females displayed increased susceptibility to AAV-shIRS1 in the novel recognition object paradigm; whereas both fernales and males show impaired performance in the T maze when infected with AAV-shIRSI compared to control. Anxiety parameters were not affected by AAV-shIRS1 infection. We observed specific fluorescence within the hilum of the dentate gyrus, in immuno-characterized parvalbumin and somatostatin neurons. AAV DJ8 did not enter astrocytes. Intense green fibers were found in the fornix, mammillary bodies, and in the medial septum indicating that hippocampal efferent had been efficiently targeted by the AAV DJ8 infection. We observed that AAV-shIRSI reduced significantly synaptophysin labeling in hippocampal-septal projections compared to controls. These results support that, small alterations in the insulin/ IGF1 pathway in specific hippocampal circuitries can underlie alterations in synaptic plasticity and affect behavior, in the absence of inflammatory conditions

Keywords Insulin resistance - IRS2 - Hippocampal dentate gyrus - Social memory - Synaptophysin - Immunofluorescence Synaptic plasticity

Electronic supplementary material The online version of this article (https://doi.org/10.1007/:00429-020-02155-x) contain supplementary material, which is available to authorized users.

Ana Maria Sínchez-Pérez sanchean 1 ujies

1 Department of Medicine, Universitat Jaume I, Castellón, Spain

2 Prosent Address: Instituto de Acuicultura de Torso de la Sal (IATSCSIC), 12595 Ribera de Cahanes, Castallón, Spain

Published online: 27 November 2020

\section{Introduction}

Insulin and IGF 1 actions are mediated by the insulin and IGFl receptors that can activate similar signaling pathways with pleiotropic actions in mammals. In the brain, both peptides have been shown to modulate cognitive function (A1Delaimy et al. 2009; Cholerton et al. 2013). The signaling cascades elicited by insulin and IGFI are almost identical and both mediated by the scaffolding proteins, the insulin receptor substrates (IRS) (Kleinridders et al. 2014; Bedse et al. 2015). There are four IRSs known isoforms, being the IRS1 and IRS2 the most abundant and studied, up to date (Bedse et al. 2015). In spite the fact that both, IR S1 and 2 have been shown to mediate insulin and IGFl effects, some

Q Springer 